\title{
Cardiac mechanomyopathy : integrating the picture from cell to beating heart
}

Citation for published version (APA):

Donker, D. W. (2008). Cardiac mechanomyopathy : integrating the picture from cell to beating heart.

[Doctoral Thesis, Maastricht University]. Universiteit Maastricht. https://doi.org/10.26481/dis.20080911dd

Document status and date:

Published: 01/01/2008

DOI:

10.26481/dis.20080911dd

Document Version:

Publisher's PDF, also known as Version of record

\section{Please check the document version of this publication:}

- A submitted manuscript is the version of the article upon submission and before peer-review. There can be important differences between the submitted version and the official published version of record.

People interested in the research are advised to contact the author for the final version of the publication, or visit the DOI to the publisher's website.

- The final author version and the galley proof are versions of the publication after peer review.

- The final published version features the final layout of the paper including the volume, issue and page numbers.

Link to publication

\footnotetext{
General rights rights.

- You may freely distribute the URL identifying the publication in the public portal. please follow below link for the End User Agreement:

www.umlib.nl/taverne-license

Take down policy

If you believe that this document breaches copyright please contact us at:

repository@maastrichtuniversity.nl

providing details and we will investigate your claim.
}

Copyright and moral rights for the publications made accessible in the public portal are retained by the authors and/or other copyright owners and it is a condition of accessing publications that users recognise and abide by the legal requirements associated with these

- Users may download and print one copy of any publication from the public portal for the purpose of private study or research.

- You may not further distribute the material or use it for any profit-making activity or commercial gain

If the publication is distributed under the terms of Article $25 \mathrm{fa}$ of the Dutch Copyright Act, indicated by the "Taverne" license above, 


\section{Cardiac}

\section{Mechanomyopathy}

Integrating the Picture from Cell to Beating Heart 



\section{Cardiac}

\section{Mechanomyopathy}

Integrating the Picture from Cell to Beating Heart

\section{PROEFSCHRIFT}

ter verkrijging van de graad van doctor aan de Universiteit Maastricht, op gezag van de Rector Magnificus, Prof. mr. G.P.M.F. Mols,

volgens het besluit van het College van Decanen,

in het openbaar te verdedigen op

donderdag 11 september 2008 om 14.00 uur

door

Dirk W. Donker

geboren op 25 september 1970 te Nordhorn (Duitsland) 


\section{Promotores}

Prof. dr. H.J.G.M. Crijns

Prof. dr. M.A. Vos Universitair Medisch Centrum Utrecht

\section{Copromotor}

Dr. P.G.A. Volders

\section{Beoordelingscommissie}

Prof. dr. M.A. Allessie Voorzitter

Prof. dr. ir. T. Arts

Prof. dr. J.F.C. Glatz

Prof. dr. A.D. McCulloch University of California, San Diego, USA

Prof. dr. W.J. Paulus Vrije Universiteit Medisch Centrum, Amsterdam en Onze-Lieve-Vrouwziekenhuis, Aalst, België 
Samen met Birgit ... voor Aaron, Eline en Marit. 
Layout and print: Datawyse Maastricht

Illustrations: Roel Spätjens

Cover design: Datawyse Maastricht

Cover illustrations: Aaron, Eline and Marit Donker, Roel Spätjens, Emile Cheriex (echocardiography), Engelbert Schins (photography)

ISBN 978-90-5278-745-9

Universitaire Pers Maastricht

๑ Dirk W. Donker, Maastricht 2008

Financial support by 'Stichting Hartsvrienden RESCAR Maastricht' and the University Medical Center Maastricht for the publication of this thesis is gratefully acknowledged. 


\section{Content}

CHAPTER 1

Introduction

CHAPTER 2

Electro-mechanical remodeling in hypertrophy

Dirk W. Donker, Harry J.G.M. Crijns, Paul G.A. Volders.

Cardiac mechano-electric feedback and arrhythmias: from pipette to patient. 2005;

Saunders - Elsevier. Peter Kohl, Frederick Sachs, Michael R. Franz (eds).

CHAPTER 3

End-diastolic myofiber stress and ejection strain increase with ventricular volume overload - serial in-vivo analyses in dogs with complete atrioventricular block Dirk W. Donker, Paul G.A. Volders, Theo Arts, Bas C.M. Bekkers, Leo Hofstra, Roel L.H.M.G. Spätjens, Jet D. Beekman, Marcel Borgers, Harry J.G.M. Crijns, Marc A. Vos.

Basic Res Cardiol. 2005;100:372-82.

Serial left-ventricular biopsy sampling using a minimally invasive trans-thoracic approach in adult dogs

Dirk W. Donker, Jos G. Maessen, Roel L.H.M.G. Spätjens, Theo van der Nagel, Monique de Jong, Frans C. Ramaekers, Harry J.G.M. Crijns, Marc A. Vos, Paul G.A. Volders.

Pflugers Arch. 2007;454:1043-51. 
Temporal patterns of electrical remodeling in canine ventricular hypertrophy: focus on $\mathrm{I}_{\mathrm{Ks}}$ downregulation and blunted B-adrenergic activation Milan Stengl, Christian Ramakers, Dirk W. Donker, Ashish Nabar, Andrew V. Rybin, Roel L.H.M.G. Spätjens, Theo van der Nagel, Will K.W.H. Wodzig, Karin R. Sipido, Gudrun Antoons, Antoon F.M. Moorman, Marc A. Vos and Paul G.A. Volders.

Cardiovasc Res. 2006;72:90-100.

\section{CHAPTER 6}

Impact of acute and enduring volume overload on mechanotransduction and cytoskeletal integrity of canine left ventricular myocardium Dirk W. Donker, Jos G. Maessen, Fons Verheyen, Frans C. Ramaekers, Roel L.H.M.G. Spätjens, Helma Kuijpers, Christian Ramakers, Paul M.H. Schiffers, Marc A. Vos, Harry J.G.M. Crijns, Paul G.A. Volders.

Am J Physiol Heart Circ Physiol. 2007;292:H2324-32.

\section{CHAPTER 7}

Non-invasive quantification of complete left-ventricular pressure-volume and stress-strain loops in the human heart - application of the CircAdapt model Dirk W. Donker, Paul G.A. Volders, Harry J.G.M. Crijns, Theo Arts.

In preparation and in part presented at the 2007 Annual Scientific Sessions of the American Heart Association.

Circulation 2007;116:I/622-I/622, 2800 Suppl. S.

CHAPTER 8

Discussion

In preparation and in part presented at the 2001 Annual Scientific Sessions of the American College of Cardiology.

J Am Coll Cardiol. 2001;37:222A-222A Suppl. A. 
CHAPTER 9

209

$\begin{array}{ll}\text { Summary } & 213\end{array}$

$\begin{array}{ll}\text { Samenvatting } & 215\end{array}$

$\begin{array}{ll}\text { Zusammenfassung } & 219\end{array}$

$\begin{array}{ll}\text { Appendix - Full colour illustrations } & 221\end{array}$

Dankwoord 231

$\begin{array}{ll}\text { Publications } & 237\end{array}$ 



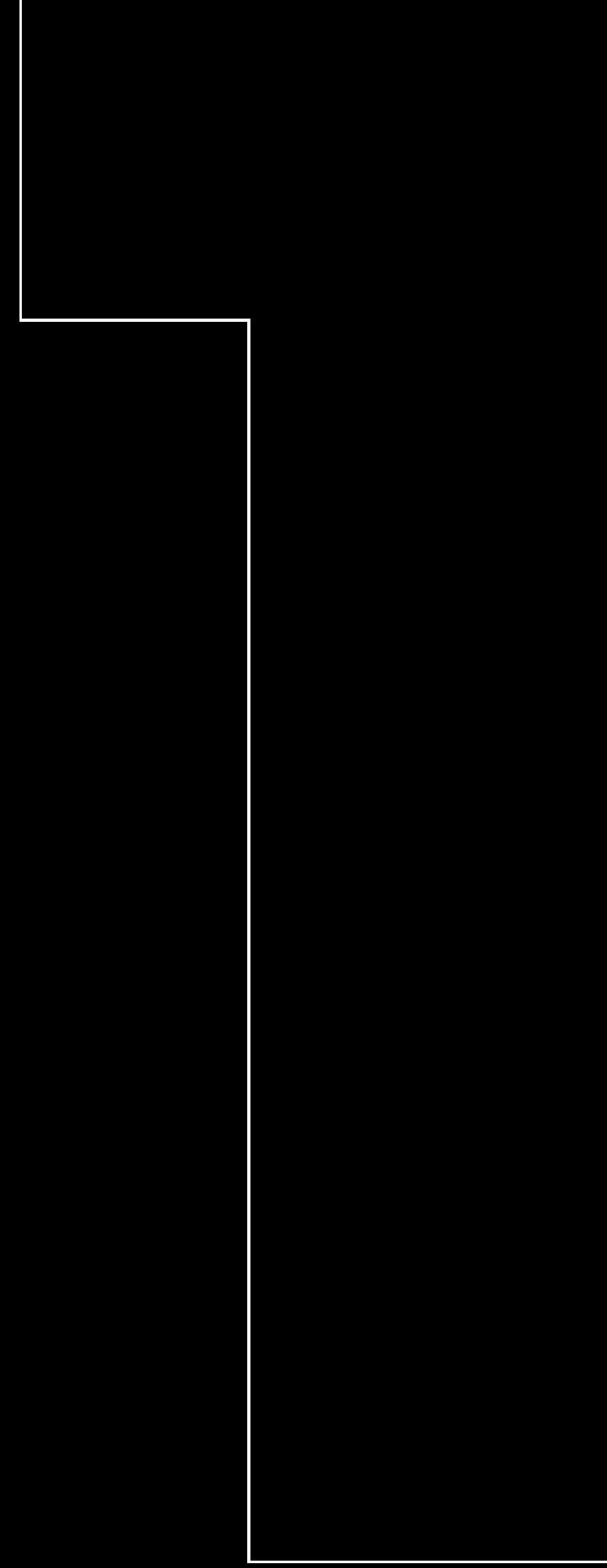



Introduction 


\section{INTRODUCTION}

Integrating the picture ... in medical terms: a comprehensive analysis of an individual phenotype. The word "phenotype" stems from the old Greek "phainein", to bring to light, and "typos", impression. Depending on the scope of the observer, the beating heart literally brings to light seemingly endless impressions. The motor of life has attracted and inspired numerous poets and visual artists throughout the history of mankind (Figure 1).

Doctors and scientists have a broad arsenal to collect impressions of the heart, ranging from interrogation of the patient ("anamnesis") to the use of high-technology diagnostics for cardiac imaging. Despite all available tools, it remains challenging in daily clinical practice to integrate all these impressions to understand the cardiac phenotype of an individual and thereby solve his or her problem.

In the studies that served as the basis for this thesis, the hearts of experimental animals and humans with ventricular hypertrophy due to different types of hemodynamic overload were phenotyped in vivo. To this aim, aspects of mechanical, contractile and electrical function, and dynamically altering myocardial structure were assessed over time.

Our understanding of overload-induced cardiac remodeling is far from complete. A decade ago, Charles J. Homcy, MD, addressed the complexity of unravelled mechanisms by wondering metaphorically 'how many switches and how many wires' would contribute to 'signaling hypertrophy' [61].

'Signaling hypertrophy: how many switches and how many wires'

Charles J. Homcy, 1998 [61]

This issue still remains to be fully elucidated. It becomes clear after analyzing the data collected in this thesis that overload-induced cardiac disease is characterized by a multitude of different aspects encompassing structural and functional myocardial remodeling processes with mutual interrelations and sharing potentially common primary stimuli. In order to consider and integrate all relevant aspects of the overloaded cardiac phenotype, we propose to designate a common denominating term, cardiac mechanomyopathy. This neologism allows to summarize all the remodeling processes as a common pathophysiological entity, thereby meeting its complex nature and aiding to unravel common underlying mechanisms. Importantly, the term indicates a primary role of mechanical stimuli for myocardial remodeling.

In this thesis, we focused on mechanical forces imposing on the heart as potential stimuli for hypertrophic remodeling due to cardiac hemodynamic overload. Although this can occur in the absence of mechanical forces, there is cumulating evidence that during hemodynamic overload mechanical forces play a primary role as stimuli for myocardial remodeling (Table 1). The identifica- 


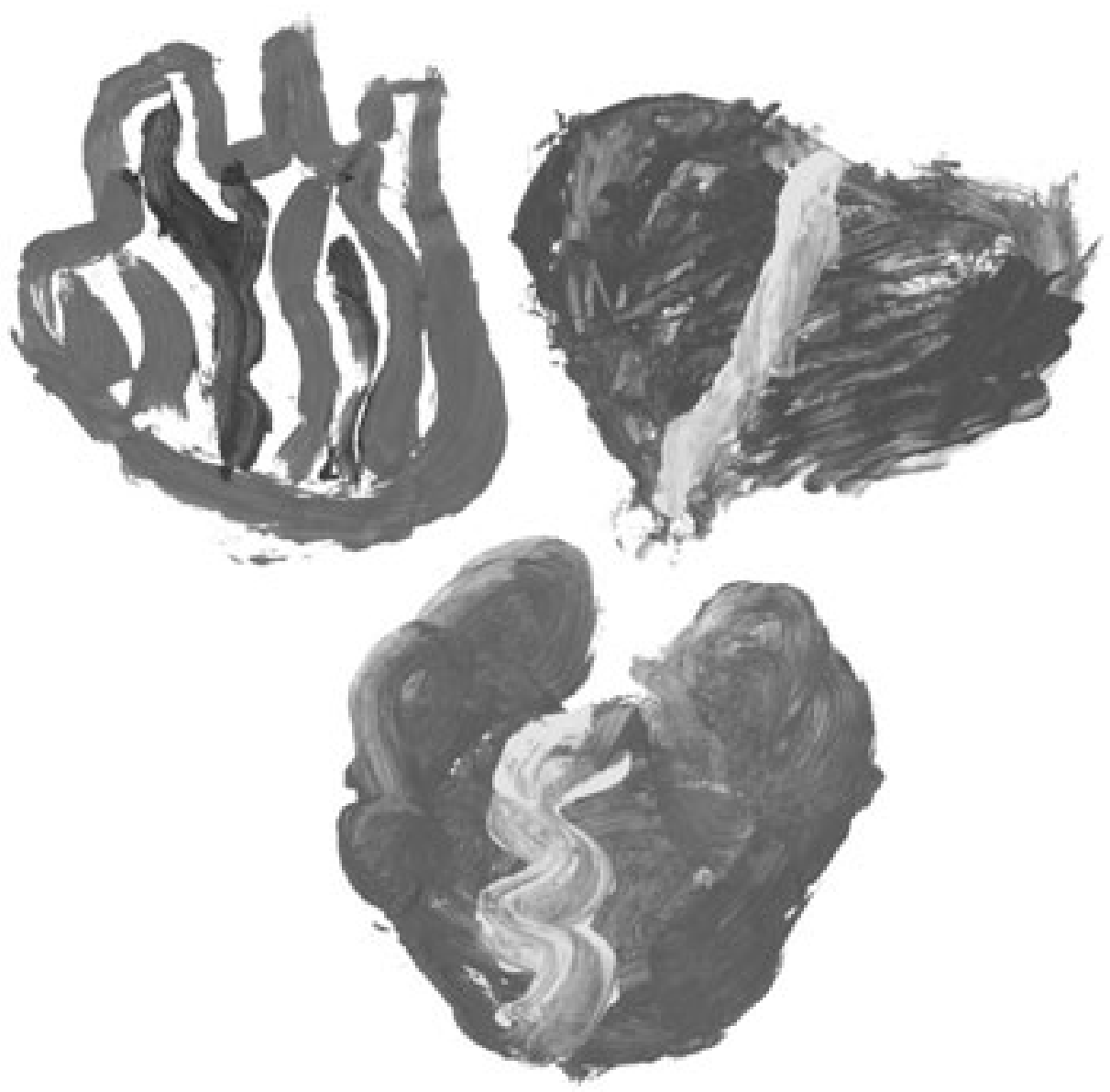

Figure 1 The heart. Artwork by Aaron (7 years), Eline (5 years) and Marit Donker (3 years). From left to right. For full colour illustration, see page 221.

tion of these primary stimuli will improve our understanding and advance novel mechanism-based diagnostic and therapeutic strategies in individual patients.

In the remainder of this Introduction the clinical relevance of cardiac phenotyping in hemodynamic overload is explained. A brief history is given of the heart and circulation, and the historic recognition that certain disease states cause cardiac overload and hypertrophy. Many aspects of the cardiac phenotype have long been known but are still not elucidated mechanistically today. 
Table 1 |PRO's and CON's. Protagonistic and antagonistic views on the primary role of mechanical forces as stimuli for hypertrophic myocardial remodeling during hemodynamic and mechanical overload.

\section{PRO's}

- Hypertrophy occurs in almost all cardiovascular diseases and related animal models in which a form of hemodynamic overload is present, e.g., arterial hypertension, valvular heart disease etc., and can be (at least partially) reversed upon withdrawal of overload. Hypertrophy can be considered a universal response to cardiac overload $[17,44,99,110,137-139]$. Hypertrophy can be induced by direct mechanical stimulation in cardiomyocyte preparations in vitro. Stretching cardiomyocyte preparations stimulates protein synthesis, induces altered gene expression without involvement of neural or humoral factors, yielding similar results as in vivo experiments $[17,19,39,44,79,83,86,94,107,108,117,125,130,137,146]$. In addition, different degrees and directions [132] of stretch, and the timing of its action within the cardiac cycle [151], result in differential hypertrophic responses, which supports primary mechanical influences on phenotypic hypertrophic changes. These aspects of phenotypical determination of cardiomyocyte hypertrophy can be observed in vivo, i.e. pressure overload results in predominant cellular thickening (addition of sarcomeres in parallel), and volume overload in predominant cellular lengthening (addition of sarcomeres in series).

- Direct non-mechanical hypertrophic stimuli, e.g., G protein - coupled receptor agonists (endothelin-1, angiotensin II, phenylephrine), which can induce hypertrophy in the absence of mechanical factors in vitro also play a role in vivo in cardiac disease with hemodynamic overload $[18,56,93,100,116,118,129,134]$. These nonmechanical stimuli are often directly coupled to mechanical stimuli acting as initiators for their release.

- Hypertrophy can be induced by hemodynamic overload in vivo even after adrenoreceptor blockade or sympathectomy [21]. Also, in isolated hearts increased hemodynamic load increases protein synthesis [78].

\section{CON's}

- Hypertrophy can be induced by direct agonist activation in the absence of mechanical stimuli in cardiomyocyte preparations in vitro, e.g., G protein - coupled receptor agonists (endothelin-1, angiotensin II, phenylephrine), growth factors (IGF-I, TGF $\beta$ ), cytokines (TNF $\alpha$ ) and hormones (thyroid hormone, insuline) $[129,134,135]$. It should be realized that the specific effect of a given hypertrophic stimulus may be the result of a complex combined action of various different pathways depending on spatial and temporal abundance of relevant proteins in the cell in question. These aspects are not necessarily determined by mechanical forces but depend on the inherent synchronization and integration of different hypertrophic pathways [130].

- Hypertrophy is importantly mediated by many other different stimuli, e.g., metabolic stress, ischemia, oxidative stress and inflammation [106,136].

- Hypertrophy can be induced by genetic modifications in experimental models under normal loading conditions by either constitutive overexpression of hypertrophy-promoter genes or knock out of hypertrophyrepressor genes.

- Hypertrophy can occur in humans with genetic defects under normal loading conditions, i.e., mutations of cytoskeletal/ sarcomeric proteins. 
In the following sections current controversies in the phenotyping of ventricular hypertrophy are discussed. An overview is provided of modern diagnostic modalities and molecular techniques that generate detailed insights on mechanosensing and mechanotransduction, and mechano-electrical links. The need for an integrated approach towards cardiac mechanomyopathy has led to the aims of the present thesis.

\section{Clinical background}

Cardiac hypertrophy is commonly defined as an increased myocardial mass of the ventricle. Although hypertrophy can affect both the left (LV) and the right ventricle, LV hypertrophy (LVH) poses a much greater problem because it affects more patients. In the general population the prevalence of LVH has been estimated to range between 15 and 20\% using different diagnostic modalities such as electrocardiography, echocardiography, magnetic resonance imaging (MRI) and autopsy $[75,90,104]$. In patients with cardiovascular disease like arterial hypertension, valvular heart disease and myocardial infarction, the prevalence of LVH is considerably higher than in the general population and reaches up to $100 \%$.

Epidemiological studies have shown that cardiac hypertrophy is associated with a high cardiovascular morbidity and mortality independent of its cause $[75,88,89,145]$. This is largely due to the fact that sustained overload will finally result in irreversible pump failure. In addition, the hypertrophied heart has an increased propensity to develop potentially fatal ventricular arrhythmias, by which the relative risk of sudden arrhythmogenic death is highest in patients with a well-preserved functional capacity $[1,141-143,154]$.

In The Netherlands, the impact of the problem of LVH can best be illustrated with data from Statistics Netherlands' and the Netherlands Heart Foundation: In 2004, a total of 136,553 people died in The Netherlands, i.e., 374 per day. Of these, 45,445 died of cardiovascular disease, i.e., 33\% of the total mortality. Of the latter group, 15,272 people died of cardiovascular disorders in which ventricular hypertrophy is generally prevalent and might play a pathological role [2].

\section{Historical notes}

William Harvey (1578-1657) was the first to describe in 1628 that the circulation consists of two separate closed circuits connected to the heart, the pulmonary and the systemic circulation; and he recognized that the heart acts as a pump enabling blood flow in arteries and veins throughout the body [55]. Harvey's description formed the basis for subsequent findings on the link between blood-flow abnormalities and cardiac enlargement, with John Mayow (1634-1679) in 1674 being among the first to publish a case of cardiac overload due to LV inflow obstruction, most likely mitral stenosis, and right-ventricular enlargement $[33,95]$. Mayow speculated already on distending forces

1 Centraal Bureau voor de Statistiek 
as potential stimulus for cardiac dilatation. Giovanni Maria Lancisi (1654-1720) was among the first who distinguished cardiac dilatation in situations of regurgitant blood flow and hypertrophy in situations of narrowing of the aortic orifice [150].

The causal relation between hemodynamic overload and cardiac enlargement was put forward by Giovanni Battista Morgagni (1682-1771) in 1761 [101]. The history of different classifications of cardiac enlargement is rather extensive and has been excellently reviewed and summarized by Katz [76]. In 1801 Jean-Nicolas Corvisart (1755-1821) distinguished in more detail ventricular hypertrophy and dilatation, which led to a general acceptance in the nineteenth century that different patterns of cardiac enlargement are the consequence of distinct hemodynamic abnormalities. Réné-Joseph-Hyacinthe Bertin (1767-1828) refined Corvisart's concept and classified ventricular hypertrophy as 'eccentric' and 'concentric' and also related the morphologic pattern to its prognostic significance. In this tradition, Francois Aran (1817-1861), was among the first to describe a compensated form of ventricular hypertrophy [5]. In the same period, Austin Flint (1812-1886) recognized many compensatory, beneficial aspects of overload-induced ventricular hypertrophy and opposed these findings to the deleterious sequelae of ventricular dilatation [37]. Intuitively, Austin Flint associated hypertrophy and heart failure with an abnormal mechanical load, yet it lasted about 100 years before these thoughts were supported by calculation of mechanical forces acting in the myocardial wall $[49,62,128]$, as outlined below. John Milner Fothergill (1841-1888) was then able to link patterns of ventricular hypertrophy to the underlying mechanical forces, i.e., 'in the distending force... hypertrophy is always combined with dilatation of the cardiac chambers; in obstruction to be overcome ... there is pure hypertrophy, usually without dilatation' [40]. Along with Austin Flint, Fothergill recognized many of the beneficial aspects of certain forms of hypertrophy. A major step forward in the understanding of cardiac pump failure and the transition from compensated hypertrophy to failure was the work of William Osler (1849-1917). He was among the first to describe the natural history of different types of overload and differentiated three stages of disease: development, full compensation and broken compensation [111]. A historical step forward in terms of mechanistic understanding was the recognition of James Mackenzie (1853-1925) that myocardial abnormalities are the major underlying cause of heart failure [92].

Then, hemodynamic and geometric assessment, i.e., intracardiac pressures and volumes, became increasingly important in heart-failure research as introduced by Otto Frank, Ernest H. Starling and Carl J. Wiggers around $1900[41,133,149]$. Owing to the introduction of cardiac catheterization, the ideas of Austin Flint and Fothergill on cardiac (de-)compensated hypertrophy and dilatation could be mechanistically confirmed by the calculation of wall stress $[49,62,128]$.

Thus, based on historical notes, mechanical influences on cardiac structure, contractile function and hemodynamics have long been addressed. In addition, electrophysiological alterations due to mechanical influences imposed on the heart have already been described more than 200 years ago. Already in 1765 it was recognized that the heart's function is sensitive to mechanical forces, when Akenside reported on the occurrence of arrhythmias after a traumatic cardiac injury [3]. In 1882, Riedinger reported on 'commotio cordis' as a trigger for arrhythmic events in the absence of tissue 
trauma [114]. In 1915, it was Bainbridge who recognized a mechanically-induced increase in heart rate [10], and in 1920 Schott reported on the 'pre-cordial percussion' as an effective therapy of Stokes-Adams attacks, which is still an element of resuscitation protocols today [131]. Finally, these and other observations led to the concept of mechano-electrical feedback, originally described by Kaufmann in 1967 as 'mechano-elektrische Rückkoppelung' [77].

\section{Phenotyping ventricular hypertrophy}

"Everyone thinks they know what "cardiac hypertrophy" is: a reactive increase in cardiac size/ myocardial mass in response to hemodynamic stress that, in humans, predisposes to early death [89]. Yet, the term "hypertrophy" has become one of the most misused and inaccurate terms in the cardiovascular basic science literature because of its nonspecificity and, as typically used, lack of mechanistic implication.... we reflect on the appropriate meanings of terms and criteria that can be used to accurately describe cardiac enlargement and myocardial growth, with the anticipation that rigorous mechanistic description of such phenotypes will result in a more coherent appreciation of the parallel and redundant processes that result in "myocardial hypertrophy."

\section{Gerald W. Dorn II, 2003 [32]}

The ventricular geometry of hypertrophied hearts is classically divided into eccentric and concentric forms based on their macroscopic structural shape $[4,49,115]$. The former is characterized by a proportional increase of ventricular cavity and wall, whereas the latter is dominated by a substantial and disproportional wall thickening in the virtual absence of chamber enlargement. The traditional paradigm is that eccentric hypertrophy occurs as a result of volume overload and its cellular basis is cardiomyocyte lengthening due to the serial addition of sarcomeres. It is opposed to a concentric geometry which results from pressure overload and exhibits an increase of the cardiomyocyte cross sectional area due to a parallel addition of sarcomeres $[47,49,4]$. Grossman and co-workers were among the first to describe the potential mechanical stimuli responsible for these differences in hypertrophic remodeling. They postulated that systolic stress imposed on the myocardium leads to wall thickening and concentric hypertrophy, which in turn could reduce or even normalize the systolic stress in a feedback loop. In volume overload Grossman et al. found that diastolic stress was likely responsible for eccentric hypertrophy, whereby the heart "grew" in a nearly physiological way, but was not able to normalize the hypertrophy-stimulating mechanical forces $[48,49]$. These findings were derived from group comparisons between normal control patients, patients with pressure overload and patients with volume overload. In that way Grossman et al. were purely descriptive as they based their conclusions exclusively on observations on systolic and diastolic LV wall stresses which partly differed among the groups. It was Karl Weber who added an important dimension to the discussion on load-dependent patterns of cardiac remodeling. Weber et al. studied extracellular-matrix remodeling and myocardial fibrosis in overload-induced cardiac disease and 
emphasized the relevance of the extracellular matrix for myocardial stiffness and contractile function $[34,148]$.

The clinical applicability of terms as 'eccentric' and 'concentric' LVH is of limited value. The differentiation of just these two types of LVH oversimplifies the many faces myocardial hypertrophy can have in humans with cardiovascular disease. Moreover, clinical studies have indicated that the pattern of hypertrophy may provide only little additional information beyond the assessment of $L V$ mass itself $[85,147]$. In addition, there is no geometry-specific therapy for eccentric volumeoverload-induced versus concentric pressure-overload-induced hypertrophy.

In disease, human hearts are exposed to complex mechanical loading conditions, often over long stretches of time, which results in a diversity of (mal)adaptational myocardial remodeling. Adequate characterization is complicated by an often unknown duration of the disease with varying ventricular loading over time, ongoing functional and structural myocardial remodeling, interference of therapeutic interventions, and genetic constitution. A lot of these aspects are beyond the scope of currently used clinical diagnostic techniques.

In order to advance more individualized, rational therapies, risk stratification and prognostic judgments, there is a need for a more detailed, mechanism-based phenotypic description of the hypertrophied ventricular myocardium in clinical medicine. Novel diagnostic approaches should be directed towards mechanistic understanding of underlying cellular remodeling processes and the causal stimuli involved [32].

\section{Phenotyping ventricular mechanical overload}

It has been recognized for many decades that cardiac overload is the principal stimulus for ventricular hypertrophy and associated myocardial remodeling in numerous cardiovascular diseases. Yet, a detailed identification of the primary stimuli that regulate hypertrophic ventricular remodeling at the level of the myocardium remains elusive. Much evidence points to a prominent role of mechanical factors as primary stimuli for cardiac hypertrophy $[38,49,105,123]$. It has also become clear that besides mechanical influences, neural- and neurohumoral stimulation, growth hormones, cytokines, alterations of metabolism and energy consumption all contribute to myocardial remodeling during ventricular hypertrophy $[68,69,102,123,124,127]$.

Cardiac mechanical load is the integrated sum of distinct elementary quantities and can basically be quantified in terms of stress and strain. This terminology is derived from physics, where stress $\sigma\left(\mathrm{kPa}=\right.$ dyne $\left./ \mathrm{cm}^{2} \times 10^{4}\right)$ is defined as a force acting on the surface of an object, and strain $\varepsilon(-)$ as the resultant deformation of this object. Strain $\varepsilon(-)$ is dimensionless and expressed as the length of the object after the deformation relative to its unstressed baseline length. By definition, positive strain represents lengthening or expansion of an object, and negative strain indicates shortening or compression. 


\section{Quantification of LV myocardial stress}

Direct measurement of stress imposed on the myocardium can be performed by insertion of technical devices, so called "force gauges", into the tissue. These gauges are able to detect the stress indirectly through deformation of a compliant element within the gauge. Yet, the invasiveness of this approach limits reliable measurement of stress due to local tissue damage and can only be applied under basic experimental conditions. Currently, there is no clinically-feasible method to measure LV myocardial stress. Novel approaches to non-invasively determine stress are described in this thesis. This is made possible by mathematical modeling of cardiac mechanics, as reviewed by McCulloch and Mazhari [98]. In computer models of cardiac mechanics, the heart is assumed to have a certain geometric shape and myocardial material properties [6-8,22-25,29,50,51,66,73,96-98]. Geometric assumptions of the LV range from simple thin-walled spheres or cylinders to more realistic thickwalled truncated ellipsoids. The most realistic models apply the three-dimensional finite element method of the heart, which is rather difficult to handle. In all models, the myocardium is assumed to be a continuous, homogenous, elastically deformable and incompressible material. In addition to assumptions on LV shape and myocardial structure, data on LV cavity pressure are incorporated. Ideally, the "model input data" on LV dimensions and pressure are simultaneously recorded to allow for proper synchronization of these two interrelated quantities to calculate myocardial stress and strain.

Most of the models published in the literature calculate LV wall stress. However, while widely used, this has inherent limitations. It is dependent on the site and direction of measurement, and absolute values generated using different models are not comparable since they do not represent true myocardial stress when compared to in-vitro/ intramyocardial recordings [7]. Nguyen et al. supported this notion by showing that fiber stresses differ significantly from wall stresses [105]. Calculation of (myo)fiber stress is preferable to LV wall stress since it represents more accurately the stresses within the myocardium. This is supported by a better comparability between calculated absolute myofiber stresses in vivo and in vitro [30], as outlined in Chapter 3. Moreover, calculation of fiber stress is found to be superior to estimates of wall stress in patients with abnormal ventricular geometry [46].

\section{Quantification of LV myocardial strain}

The invasive gold standard for direct measurement of strain is the intramyocardial application of sonomicrometric piezoelectric crystals. Although this technique is used in acute and chronic experiments to validate non-invasive modalities assessing strain, it is limited by local tissue reaction and is not applicable to humans due to its invasiveness.

Non-invasive assessment of strain is possible using different approaches, including computer modeling, Doppler echocardiography and MRI [59]. Computer modeling of cardiac mechanics has recently been reviewed [98] and is shortly discussed above. The clinical applicability is limited due to the use of complex algorithms. Recent technical developments in echocardiography allow 
assessment of regional myocardial deformation by strain and strain-rate imaging $[26,27]$. To this aim, tissue-Doppler imaging using real-time echocardiography is applied.

Assessment of strain and strain rate (i.e., the temporal derivative of strain being a measure of rate of deformation) by tissue-Doppler imaging (TDI) is mainly regarded as a method to measure regional systolic myocardial function [57], i.e., either LV long-axis shortening strain (longitudinal strain), short-axis thickening strain (radial strain) or short-axis shortening strain (circumferential strain). This technique provides continuous information on changes in regional dimensions. Important limitations are the inherent angle dependency of the ultrasound beam relative to the myocardial wall and the necessity of sufficient image quality, which is critical for the application of this imaging modality $[26,59,84,144]$. Magnetic Resonance Imaging (MRI) tagging is currently accepted as the non-invasive diagnostic reference modality to assess myocardial strain [113]. The clinical use of MRI tagging as a measure of regional contractility is still limited. The data analysis is time consuming and overall relatively costly in comparison to echocardiographic techniques. Moreover, MRI is not available at the bedside. This precludes its use for many routine purposes in a clinical setting $[16,59,91,113,152]$.

\section{Importance of assessing LV myocardial stress and strain}

From in-vitro and in-vivo studies in animal models and humans with mechanical overload it is well recognized that more than two mechanical stimuli, as classically described [49], are causally involved in hypertrophic ventricular remodeling [60]. The traditional assumptions as e.g., the dominant role of systolic load during pressure-overload hypertrophy have not been unequivocally supported: Nguyen et al. [105] suggested that end-diastolic stress is more likely the stimulus for pressure overload hypertrophy than peak systolic stress. This in-vivo finding indicates that the causal role of mechanical stimuli for hypertrophy is much more complex than the traditionally assumed dichotomy of concentric versus eccentric hypertrophic patterns as proposed by Grossman et al., who suggested that hypertrophy develops to normalize systolic but not diastolic wall stress [49]. Many mechanical stimuli are likely involved as hypertrophic stimuli, which deserves further analysis as pointed out by Holmes [60]. In-vitro experiments have been performed to clarify this complexity. In cardiomyocyte preparations, it has been shown that the spatial orientation [132] of externally applied mechanical load, with respect to the cellular axes and its timing within the cardiac cycle [151], differentially influence the biological responses of cardiomyocytes. Also, external loads as applied to passively stretched cardiomyocytes [94], should be contrasted to internal loads as occurring in field-stimulated actively contracting cells [70]. In the light of this in-vitro information, many temporal and spatial aspects of myocardial load may form relevant stimuli for hypertrophic ventricular remodeling as recently reviewed $[60,109]$. Importantly, stress and strain act at the cellular level within the ventricular wall and determine myocardial remodeling [65]. The identification of mechanical stimuli responsible for different aspects of these remodeling processes will likely increase mechanistic understanding. The translation of these basic insights into clinical practice 
would allow the development of diagnostic, prognostic and therapeutic strategies focusing on the underlying mechanisms involved in hypertrophic remodeling.

\section{Mechanosensing and mechanotransduction}

It is well-established that mechanical forces directly imposed on cardiomyocytes in vitro evoke various cellular responses [123], as illustrated in Figure 2. In addition, in-vivo studies in animal models and humans with cardiac overload have described numerous effects on the myocardium. The underlying molecular cascades have been studied extensively and different major pathways, including hormones, vasoactive substances, cytokines which are involved in the induction of myocardial hypertrophy have been reported in the literature, as recently reviewed $[17,19,20,28$, $31,35,36,39,43,44,48,53,54,61,63,65,67,70,80,86,118,137]$. However, mechanistic insights about cellular mechanotransduction are still scarce and it is currently unclear how mechanical signals are sensed and transduced by cardiomyocytes $[8,9]$.

Mechanotransduction can be defined as 'the process of converting physical forces into biochemical signals and integrating these signals into the cellular responses' [65] or as 'the biochemical response of cells to mechanical stimulation' [86]. It should be noted that many authors describe these phenomena as mechanosensing [11]. In the literature, the distinction between the terms mechanotransduction and mechanosensing is not always clear and differences are often ill-defined. Mechanosensing should be reserved only for the initial, proximal cellular response to an externally applied force [80].

Although it is currently unknown how a cardiomyocyte actually senses mechanical load, i.e., stress and strain, much evidence suggests a direct load-sensing mechanism as reviewed by Omens [109]. However, a cellular load-sensor is not definitely identified. Various cellular components have been implicated in mechanosensing and -transduction. It is likely that the cell is capable of detecting stress indirectly as strain by the deformation of cellular elements. Several cytoskeletal elements and downstream protein cascades have been characterized as relevant for mechanotransduction. Furthermore, direct functional connections between membrane-bound mechanosensors and the nucleus have been reported which have been considered to regulate transcription $[45,67,71,118$ 122,140].

\section{Mechano-electrical links}

It has long been recognized that mechanical forces can influence the electrical function of the heart as mentioned above (see Historical notes). The contemporary knowledge of mechano-electrical phenomena has recently been summarized and extensively reviewed [82]. Clinical and experimental aspects of mechano-electrical links during chronic hemodynamic overload and ventricular hypertrophy are described in Chapter 2 [82]. Importantly, chronic mechano-electrical phenomena must be distinguished from acute effects. The latter applies to electrophysiological effects of acute 


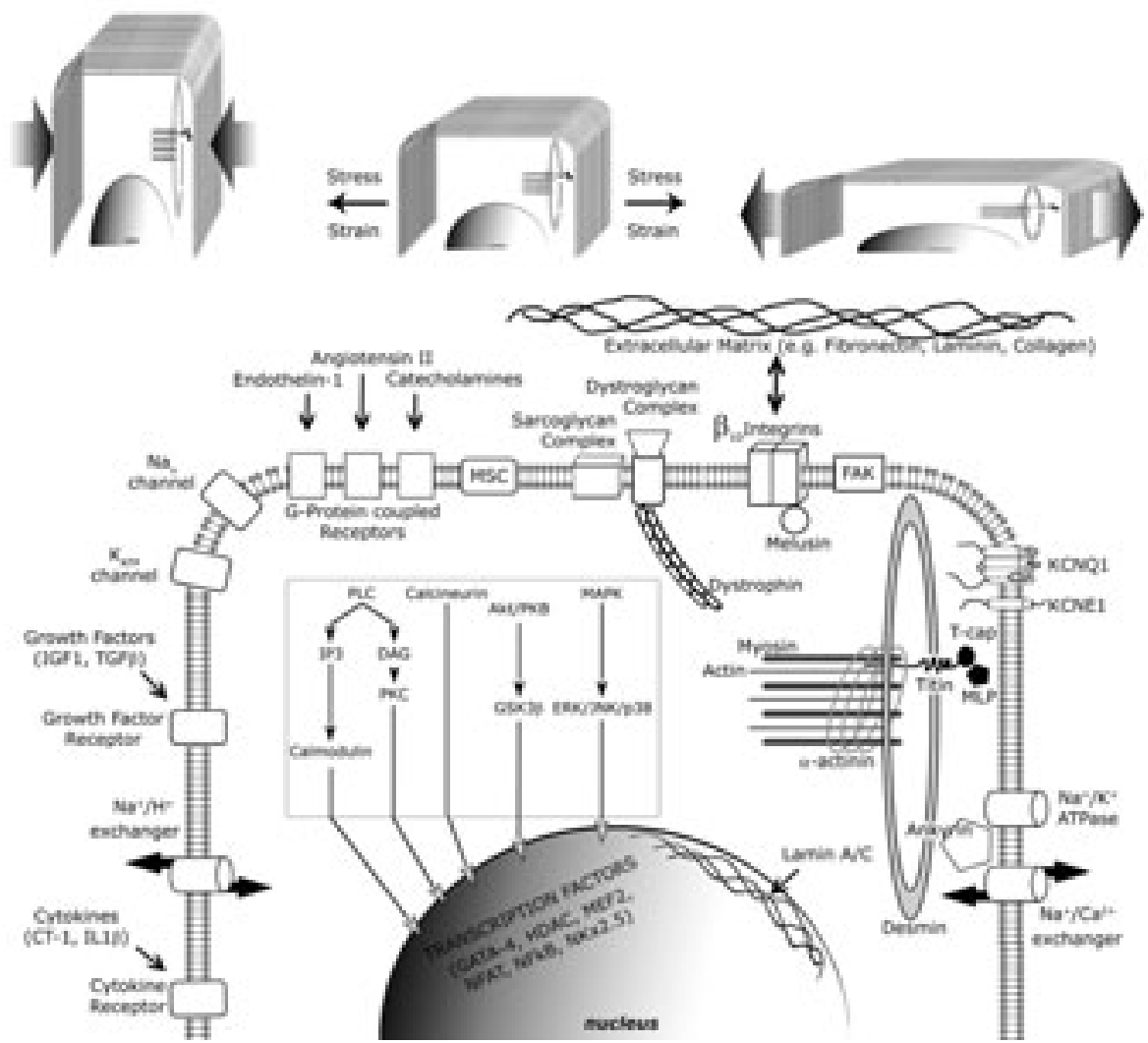

Figure $2 /$ Mechanosensing and mechanotransduction in cardiomyocytes. Simplified scheme incorporating major cardiomyocyte mechanosensing and mechanotransduction elements reported in the literature $[11-15,34-36,39,52,58,63,67,74,80,81,86,87,103,107-$ $109,112,117-122,126,134,137,138,153]$. Note miniaturized schemes on top: illustration of mechanical forces, i.e., stress and strain, imposed on cardiomyocyte that dynamically alter the cellular shape in all directions. This may result in increased broadening and/or elongation of the cell within the cardiac cycle. Abbreviations: Akt/PKB, Akt, protein kinase B; CaMK, $\mathrm{Ca}^{2+} /$ calmodulin-dependent protein kinase, or CaM kinase; CT-1, cardiotrophin; DAG, diacylglycerol; ERK, extracellular signal-regulated kinases, mitogen-activated protein kinases; FAK, focal adhesion kinase; GATA-4, cardiac transcription factor, zinc finger-containing transcription factor; HDAC, histone deacetylase; IGF1, insuline-like growth factor; IL1ß, interleukin; IP3, inositol 1,4,5-trisphosphate; JNK, C-Jun N-terminal kinases, mitogen-activated protein kinases; KCNE1, or minK, $\beta$-subunit of $\mathrm{I}_{\mathrm{Ks}}$ channel (slowly-activating delayed-rectifier $\mathrm{K}^{+}$channel); KCNQ1, or KVLQT1, $\beta$-subunit of $I_{\text {Ks }}$ channel; MAPK, mitogen-activated protein kinases; MEF2, cardiac transcription factor; MSC, mechanosensitive channels including stretch-activated ion channels; MLP, muscle LIM protein; $\mathrm{Na}_{\mathrm{v}}$, voltage-gated sodium channels; NFAT, nuclear factor of activated T-cells, cardiac transcription factor; NF- $\kappa$ B, nuclear factor-kappa B; Nkx 2.5, cardiac transcription factor, vertebrate homologue of tinman transcription factor; p38, mitogen-activated protein kinase; PKC, protein kinase C; PLC, phospholipase C; T-cap, telethonin; TGF $\beta$, transforming growth factor beta. 
stretch onto the myocardium [42], which have been well documented and found to be principally based on the action of stretch-activated ion channels $[64,120]$. Chronic mechano-electrical phenomena, have also been described under the term electrical remodeling. Electrical remodeling could be defined as a 'persistent change in the electrophysiological properties of myocardium' [72] and it is mostly used to describe long-term remodeling of ion channels and other electrogenic ion transporters. The mechanisms that link mechanics to electrical remodeling in a chronic setting are still largely unexplored and deserve further investigation as outlined in Chapter 2 [82].

\section{AIMS OF THE THESIS}

In the studies conducted for this thesis, we investigated mechanical overload-induced ventricular hypertrophy by integrating functional and structural aspects of the remodeling heart over time. To this aim, we developed novel approaches and techniques that allow to 1) quantify mechanical forces acting on the ventricular myocardium by minimally-invasive, and eventually non-invasive means, 2) sample ventricular myocardial tissue from in-situ beating hearts in chronic experiments, and thereby 3 ) integrate mechanical, functional and molecular-biological data to gain more mechanistic insights on mechano-functional and mechano-structural links. Finally, we have translated our experimental experience and insights into clinical studies. 


\section{REFERENCES}

1. Effect of metoprolol CR/XL in chronic heart failure: Metoprolol CR/XL Randomised Intervention Trial in Congestive Heart Failure (MERIT-HF). Lancet 353: 2001-2007, 1999.

2. Hart- en vaatziekten in Nederland 2006, cijfers over ziekte en sterfte. Uitgave van de Nederlandse Hartstichting. Hoofdstuk 1: Hart- en vaatziekten in Nederland. Meest recente gegevens over het jaar 2004, 2006.

3. Akenside M. An account of a blow upon the heart, and of its effects. Philos Trans R Soc Lond 53: 353-355, 1765.

4. Anversa P, Ricci R, and Olivetti G. Quantitative structural analysis of the myocardium during physiologic growth and induced cardiac hypertrophy: a review. J Am Coll Cardiol 7: 1140-1149, 1986.

5. Aran FA. Practical Manual of the Diseases of the Heart and Great Vessels. tr. Harris WA. Barrington and Haswell, Philadelphia, PA., 1843.

6. Arts T, Bovendeerd P, Delhaas T, and Prinzen F. Modeling the relation between cardiac pump function and myofiber mechanics. J Biomech 36: 731-736, 2003.

7. Arts T, Bovendeerd PH, Prinzen FW, and Reneman RS. Relation between left ventricular cavity pressure and volume and systolic fiber stress and strain in the wall. Biophys J 59: 93-102, 1991.

8. Arts T, Prinzen FW, Snoeckx LH, and Reneman RS. A model approach to the adaptation of cardiac structure by mechanical feedback in the environment of the cell. Adv Exp Med Biol 382: 217-228, 1995.

9. Arts T, Prinzen FW, Snoeckx LH, Rijcken JM, and Reneman RS. Adaptation of cardiac structure by mechanical feedback in the environment of the cell: a model study. Biophys J 66: 953-961, 1994.

10. Bainbridge FA. The influence of venous filling upon the rate of the heart. J Physiol 50: 65-84, 1915.

11. Barki-Harrington $L$ and Rockman HA. Sensing heart stress. Nat Med 9: 19-20, 2003.

12. Bennett PB. Anchors Aweigh!: ion channels, cytoskeletal proteins, and cellular excitability. Circ Res 86: 367-368, 2000.

13. Bergmann MW and De Windt LJ. Linking cardiac mechanosensing at the sarcomere M-band, nuclear factor kappaB signaling, and cardiac remodeling. Hypertension 49: 1225-1227, 2007.

14. Bienengraeber $M$, Hodgson $D M$, Zingman $L V$, Alekseev $A E$, and Terzic $A$. Identity and function of cardiac K(ATP) channels. J Mol Cell Cardiol 35: 433-435, 2003.

15. Bloom S, Lockard VG, and Bloom M. Intermediate filament-mediated stretch-induced changes in chromatin: a hypothesis for growth initiation in cardiac myocytes. J Mol Cell Cardiol 28: 2123-2127, 1996.

16. Buchalter MB, Weiss JL, Rogers WJ, Zerhouni EA, Weisfeldt ML, Beyar R, and Shapiro EP. Noninvasive quantification of left ventricular rotational deformation in normal humans using magnetic resonance imaging myocardial tagging. Circulation 81: 1236-1244, 1990.

17. Chien KR. Stress pathways and heart failure. Cell 98: 555-558, 1999.

18. Clerk A, Cullingford TE, Fuller SJ, Giraldo A, Markou T, Pikkarainen S, and Sugden PH. Signaling pathways mediating cardiac myocyte gene expression in physiological and stress responses. J Cell Physiol 212: 311-322, 2007. 
19. Cooper Gt. Basic determinants of myocardial hypertrophy: a review of molecular mechanisms. Annu Rev Med 48: 13-23, 1997.

20. Cooper Gt, Kent RL, and Mann DL. Load induction of cardiac hypertrophy. J Mol Cell Cardiol 21 Suppl 5: 11-30, 1989.

21. Cooper Gt, Kent RL, Uboh CE, Thompson EW, and Marino TA. Hemodynamic versus adrenergic control of cat right ventricular hypertrophy. J Clin Invest 75: 1403-1414, 1985.

22. Costa KD, Hunter PJ, Rogers JM, Guccione JM, Waldman LK, and McCulloch AD. A three-dimensional finite element method for large elastic deformations of ventricular myocardium: I--Cylindrical and spherical polar coordinates. J Biomech Eng 118: 452-463, 1996.

23. Costa KD, Hunter PJ, Wayne JS, Waldman LK, Guccione JM, and McCulloch AD. A three-dimensional finite element method for large elastic deformations of ventricular myocardium: Il--Prolate spheroidal coordinates. J Biomech Eng 118: 464-472, 1996.

24. Costa KD, May-Newman K, Farr D, O'Dell WG, McCulloch AD, and Omens JH. Three-dimensional residual strain in midanterior canine left ventricle. Am J Physiol 273: H1968-1976, 1997.

25. Costa KD, Takayama Y, McCulloch AD, and Covell JW. Laminar fiber architecture and three-dimensional systolic mechanics in canine ventricular myocardium. Am J Physiol 276: H595-607, 1999.

26. D'Hooge J, Heimdal A, Jamal F, Kukulski T, Bijnens B, Rademakers F, Hatle L, Suetens P, and Sutherland GR. Regional strain and strain rate measurements by cardiac ultrasound: principles, implementation and limitations. Eur J Echocardiogr 1: 154-170, 2000.

27. D'Hooge J, Herbots L, and Sutherland GR. Quantitative assessment of intrinsic regional myocardial deformation by Doppler strain rate echocardiography in humans. Circulation 107: e49; author reply e49, 2003.

28. Dambrin G, Bonnet D, and Swynghedauw B. Biology of cardiac overload. A review. J Heart Valve Dis 3: 627-633, 1994.

29. DeAnda A, Jr., Komeda M, Moon MR, Green GR, Bolger AF, Nikolic SD, Daughters GT, 2nd, and Miller DC. Estimation of regional left ventricular wall stresses in intact canine hearts. Am J Physiol 275: H18791885, 1998.

30. Donker DW, Volders PG, Arts T, Bekkers BC, Hofstra L, Spatjens RL, Beekman JD, Borgers M, Crijns HJ, and Vos MA. End-diastolic myofiber stress and ejection strain increase with ventricular volume overload Serial in-vivo analyses in dogs with complete atrioventricular block. Basic Res Cardiol 100: 372-382, 2005.

31. Dorn GW, 2nd and Force T. Protein kinase cascades in the regulation of cardiac hypertrophy. J Clin Invest 115: 527-537, 2005.

32. Dorn GW, 2nd, Robbins J, and Sugden PH. Phenotyping hypertrophy: eschew obfuscation. Circ Res 92: $1171-1175,2003$.

33. East T. Lecture 4: Failure of the circulation and its treatment. The Story of Heart Disease Dawson and Sons, London, 1958.

34. Eghbali M and Weber KT. Collagen and the myocardium: fibrillar structure, biosynthesis and degradation in relation to hypertrophy and its regression. Mol Cell Biochem 96: 1-14, 1990.

35. Epstein ND and Davis JS. Sensing stretch is fundamental. Cell 112: 147-150, 2003. 
36. Ervasti JM. Costameres: the Achilles' heel of Herculean muscle. J Biol Chem 278: 13591-13594, 2003.

37. Flint A. Diseases of the Heart, 2nd Ed. HC Lea, Philadelphia, PA, 1870., 1870.

38. Florenzano $\mathrm{F}$ and Glantz SA. Left ventricular mechanical adaptation to chronic aortic regurgitation in intact dogs. Am J Physiol 252: H969-984, 1987.

39. Force $T$, Michael $A$, Kilter $H$, and Haq S. Stretch-activated pathways and left ventricular remodeling. J Card Fail 8: S351-358, 2002.

40. Fothergill JM. The Heart and Its Diseases, 2nd Ed. Lindsay \& Blakiston, Philadelphia. 1879.

41. Frank 0. Zur Dynamik des Herzmuskels. Z Bio/ 32: 370-447, 1895.

42. Franz MR, Cima R, Wang D, Profitt D, and Kurz R. Electrophysiological effects of myocardial stretch and mechanical determinants of stretch-activated arrhythmias. Circulation 86: 968-978, 1992.

43. Frey N, Katus HA, Olson EN, and Hill JA. Hypertrophy of the heart: a new therapeutic target? Circulation 109: 1580-1589, 2004.

44. Frey N and OIson EN. Cardiac hypertrophy: the good, the bad, and the ugly. Annu Rev Physiol 65: 45-79, 2003.

45. Galbraith CG and Sheetz MP. Forces on adhesive contacts affect cell function. Curr Opin Cell Biol 10: 566-571, 1998.

46. Gentles TL and Colan SD. Wall stress misrepresents afterload in children and young adults with abnormal left ventricular geometry. J Appl Physio/ 92: 1053-1057, 2002.

47. Gerdes AM, Campbell SE, and Hilbelink DR. Structural remodeling of cardiac myocytes in rats with arteriovenous fistulas. Lab Invest 59: 857-861, 1988.

48. Grossman W. Cardiac hypertrophy: useful adaptation or pathologic process? Am J Med 69: 576-584, 1980.

49. Grossman W, Jones D, and McLaurin LP. Wall stress and patterns of hypertrophy in the human left ventricle. J Clin Invest 56: 56-64, 1975.

50. Guccione JM, Costa KD, and McCulloch AD. Finite element stress analysis of left ventricular mechanics in the beating dog heart. J Biomech 28: 1167-1177, 1995.

51. Guccione JM, McCulloch AD, and Waldman LK. Passive material properties of intact ventricular myocardium determined from a cylindrical model. J Biomech Eng 113: 42-55, 1991.

52. Gupta S, Das B, and Sen S. Cardiac hypertrophy: mechanisms and therapeutic opportunities. Antioxid Redox Signal 9: 623-652, 2007.

53. Haq S, Choukroun G, Lim H, Tymitz KM, del Monte F, Gwathmey J, Grazette L, Michael A, Hajjar R, Force $T$, and Molkentin JD. Differential activation of signal transduction pathways in human hearts with hypertrophy versus advanced heart failure. Circulation 103: 670-677, 2001.

54. Hardt SE and Sadoshima J. Negative regulators of cardiac hypertrophy. Cardiovasc Res 63: 500-509, 2004.

55. Harvey W. Exercitatio Anatomica de Motu Cordis et Sanguinis in Animalibus., 1628. 
56. Hefti MA, Harder BA, Eppenberger HM, and Schaub MC. Signaling pathways in cardiac myocyte hypertrophy. J Mol Cell Cardiol 29: 2873-2892, 1997.

57. Heimdal A, Stoylen A, Torp $H$, and Skjaerpe T. Real-time strain rate imaging of the left ventricle by ultrasound. J Am Soc Echocardiogr 11: 1013-1019, 1998.

58. Hein S, Kostin S, Heling A, Maeno Y, and Schaper J. The role of the cytoskeleton in heart failure. Cardiovasc Res 45: 273-278, 2000.

59. Herbots L, Maes F, D'Hooge J, Claus P, Dymarkowski S, Mertens P, Mortelmans L, Bijnens B, Bogaert J, Rademakers FE, and Sutherland GR. Quantifying myocardial deformation throughout the cardiac cycle: a comparison of ultrasound strain rate, grey-scale M-mode and magnetic resonance imaging. Ultrasound Med Biol 30: 591-598, 2004.

60. Holmes JW. Candidate mechanical stimuli for hypertrophy during volume overload. J Appl Physiol 97: 1453-1460, 2004

61. Homcy CJ. Signaling hypertrophy: how many switches, how many wires. Circulation 97: 1890-1892, 1998.

62. Hood WP, Jr., Rackley CE, and Rolett EL. Wall stress in the normal and hypertrophied human left ventricle. Am J Cardiol 22: 550-558, 1968.

63. Hoshijima M. Mechanical stress-strain sensors embedded in cardiac cytoskeleton: Z disk, titin, and associated structures. Am J Physiol Heart Circ Physiol 290: H1313-1325, 2006.

64. $\mathrm{Hu} \mathrm{H}$ and Sachs F. Stretch-activated ion channels in the heart. J Mol Cell Cardiol 29: 1511-1523, 1997.

65. Huang $\mathrm{H}, \mathrm{Kamm} \mathrm{RD}$, and Lee RT. Cell mechanics and mechanotransduction: pathways, probes, and physiology. Am J Physiol Cell Physiol 287: C1-11, 2004.

66. Hunter PJ, McCulloch AD, and ter Keurs HE. Modelling the mechanical properties of cardiac muscle. Prog Biophys Mol Biol 69: 289-331, 1998.

67. Ingber DE. Cellular basis of mechanotransduction. Biol Bull 194: 323-325; discussion 325-327, 1998.

68. Ito H, Hirata Y, Adachi S, Tanaka M, Tsujino M, Koike A, Nogami A, Murumo F, and Hiroe M. Endothelin-1 is an autocrine/paracrine factor in the mechanism of angiotensin II-induced hypertrophy in cultured rat cardiomyocytes. J Clin Invest 92: 398-403, 1993.

69. Ito $H$, Hiroe M, Hirata $Y$, Tsujino M, Adachi S, Shichiri M, Koike A, Nogami A, and Marumo F. Insulin-like growth factor-I induces hypertrophy with enhanced expression of muscle specific genes in cultured rat cardiomyocytes. Circulation 87: 1715-1721, 1993.

70. Ivester CT, Kent RL, Tagawa H, Tsutsui H, Imamura T, Cooper Gt, and McDermott PJ. Electrically stimulated contraction accelerates protein synthesis rates in adult feline cardiocytes. Am J Physiol 265: H666-674, 1993.

71. Janmey PA. The cytoskeleton and cell signaling: component localization and mechanical coupling. Physiol Rev 78: 763-781, 1998.

72. Jeyaraj D, Wilson LD, Zhong J, Flask C, Saffitz JE, Deschenes I, Yu X, and Rosenbaum DS. Mechanoelectrical feedback as novel mechanism of cardiac electrical remodeling. Circulation 115: 3145-3155, 2007.

73. Jones JV, Serafi AS, and James MA. Wall stress and the heart. J Cardiovasc Risk 7: 159-161, 2000.

74. Kamkin A and Kiseleva I. Mechanosensitivity in Cells and Tissues. Academia, Moscow, 2005. 
75. Kannel WB, Gordon T, and Offutt D. Left ventricular hypertrophy by electrocardiogram. Prevalence, incidence, and mortality in the Framingham study. Ann Intern Med 71: 89-105, 1969.

76. Katz AM. Evolving concepts of heart failure: cooling furnace, malfunctioning pump, enlarging muscle. Part II: Hypertrophy and dilatation of the failing heart. J Card Fail 4: 67-81, 1998.

77. Kaufmann R and Theophile U. [Autonomously promoted extension effect in Purkinje fibers, papillary muscles and trabeculae carneae of rhesus monkeys]. Pflugers Arch Gesamte Physiol Menschen Tiere 297: 174-189, 1967.

78. Kira Y, Kochel PJ, Gordon EE, and Morgan HE. Aortic perfusion pressure as a determinant of cardiac protein synthesis. Am J Physiol 246: C247-258, 1984.

79. Kira Y, Nakaoka T, Hashimoto E, Okabe F, Asano S, and Sekine I. Effect of long-term cyclic mechanical load on protein synthesis and morphological changes in cultured myocardial cells from neonatal rat. Cardiovasc Drugs Ther 8: 251-262, 1994.

80. Knoll R, Hoshijima M, and Chien K. Cardiac mechanotransduction and implications for heart disease. J Mol Med 81: 750-756, 2003.

81. Knoll R, Hoshijima M, Hoffman HM, Person V, Lorenzen-Schmidt I, Bang ML, Hayashi T, Shiga N, Yasukawa H, Schaper W, McKenna W, Yokoyama M, Schork NJ, Omens JH, McCulloch AD, Kimura A, Gregorio CC, Poller W, Schaper J, Schultheiss HP, and Chien KR. The cardiac mechanical stretch sensor machinery involves a $Z$ disc complex that is defective in a subset of human dilated cardiomyopathy. Cell 111: 943-955, 2002.

82. Kohl P, Sachs F, and Franz MR. Cardiac mechano-electric feedback and arrhythmias: from pipette to patient. Elsevier Saunders, Philadelphia, PA. 2005.

83. Komuro I, Kaida T, Shibazaki Y, Kurabayashi M, Katoh Y, Hoh E, Takaku F, and Yazaki Y. Stretching cardiac myocytes stimulates protooncogene expression. J Biol Chem 265: 3595-3598, 1990.

84. Kowalski M, Kukulski T, Jamal F, D'Hooge J, Weidemann F, Rademakers F, Bijnens B, Hatle L, and Sutherland GR. Can natural strain and strain rate quantify regional myocardial deformation? A study in healthy subjects. Ultrasound Med Biol 27: 1087-1097, 2001.

85. Krumholz HM, Larson M, and Levy D. Prognosis of left ventricular geometric patterns in the Framingham Heart Study. J Am Coll Cardiol 25: 879-884, 1995.

86. Lammerding J, Kamm RD, and Lee RT. Mechanotransduction in cardiac myocytes. Ann N Y Acad Sci 1015: 53-70, 2004.

87. Lammerding J, Schulze PC, Takahashi T, Kozlov S, Sullivan T, Kamm RD, Stewart CL, and Lee RT. Lamin A/C deficiency causes defective nuclear mechanics and mechanotransduction. J Clin Invest 113: 370-378, 2004.

88. Levy D, Anderson KM, Savage DD, Balkus SA, Kannel WB, and Castelli WP. Risk of ventricular arrhythmias in left ventricular hypertrophy: the Framingham Heart Study. Am J Cardiol 60: 560-565, 1987.

89. Levy D, Labib SB, Anderson KM, Christiansen JC, Kannel WB, and Castelli WP. Determinants of sensitivity and specificity of electrocardiographic criteria for left ventricular hypertrophy. Circulation 81: 815-820, 1990.

90. Levy D, Savage DD, Garrison RJ, Anderson KM, Kannel WB, and Castelli WP. Echocardiographic criteria for left ventricular hypertrophy: the Framingham Heart Study. Am J Cardiol 59: 956-960, 1987. 
91. MacGowan GA, Shapiro EP, Azhari H, Siu CO, Hees PS, Hutchins GM, Weiss JL, and Rademakers FE. Noninvasive measurement of shortening in the fiber and cross-fiber directions in the normal human left ventricle and in idiopathic dilated cardiomyopathy. Circulation 96: 535-541, 1997.

92. Mackenzie J. Diseases of the Heart. Oxford University Press, London., 1908.

93. MacLellan WR and Schneider MD. Genetic dissection of cardiac growth control pathways. Annu Rev Physiol 62: 289-319, 2000.

94. Mann DL, Kent RL, and Cooper Gt. Load regulation of the properties of adult feline cardiocytes: growth induction by cellular deformation. Circ Res 64: 1079-1090, 1989.

95. Mayow J. Tractus Quinque. 1674.

96. McCulloch A, Bassingthwaighte J, Hunter P, and Noble D. Computational biology of the heart: from structure to function. Prog Biophys Mol Biol 69: 153-155, 1998.

97. McCulloch AD. Functionally and structurally integrated computational modeling of ventricular physiology. Jpn J Physiol 54: 531-539, 2004.

98. McCulloch $A D$ and Mazhari R. Regional myocardial mechanics: integrative computational models of flow-function relations. J Nucl Cardiol 8: 506-519, 2001.

99. Modesti PA, Vanni S, Bertolozzi I, Cecioni I, Polidori G, Paniccia R, Bandinelli B, Perna A, Liguori P, Boddi M, Galanti G, and Serneri GG. Early sequence of cardiac adaptations and growth factor formation in pressure- and volume-overload hypertrophy. Am J Physiol Heart Circ Physiol 279: H976-985, 2000.

100. Molkentin JD and Dorn IG, 2nd. Cytoplasmic signaling pathways that regulate cardiac hypertrophy. Annu Rev Physiol 63: 391-426, 2001.

101. Morgagni GB. De sedibus et causis morborum per anatomen indagatis (De sedibus). 1761.

102. Morgan HE and Baker KM. Cardiac hypertrophy. Mechanical, neural, and endocrine dependence. Circulation 83: 13-25, 1991.

103. Morris CE and Juranka PF. Nav channel mechanosensitivity: activation and inactivation accelerate reversibly with stretch. Biophys J 93: 822-833, 2007.

104. Myerson SG, Bellenger NG, and Pennell DJ. Assessment of left ventricular mass by cardiovascular magnetic resonance. Hypertension 39: 750-755, 2002.

105. Nguyen TN, Chagas AC, and Glantz SA. Left ventricular adaptation to gradual renovascular hypertension in dogs. Am J Physiol 265: H22-38, 1993.

106. Nian $M$, Lee $P$, Khaper $N$, and Liu P. Inflammatory cytokines and postmyocardial infarction remodeling. Circ Res 94: 1543-1553, 2004.

107. Ogawa E, Saito Y, Harada M, Kamitani S, Kuwahara K, Miyamoto Y, Ishikawa M, Hamanaka I, Kajiyama N, Takahashi N, Nakagawa O, Masuda I, Kishimoto I, and Nakao K. Outside-in signalling of fibronectin stimulates cardiomyocyte hypertrophy in cultured neonatal rat ventricular myocytes. J Mol Cell Cardiol 32: 765-776, 2000.

108. Ogawa E, Saito Y, Kuwahara K, Harada M, Miyamoto Y, Hamanaka I, Kajiyama N, Takahashi N, Izumi T, Kawakami R, Kishimoto I, Naruse Y, Mori N, and Nakao K. Fibronectin signaling stimulates BNP gene transcription by inhibiting neuron-restrictive silencer element-dependent repression. Cardiovasc Res 53: 451-459, 2002. 
109. Omens JH. Stress and strain as regulators of myocardial growth. Prog Biophys Mol Biol 69: 559-572, 1998.

110. Opie LH, Commerford PJ, Gersh BJ, and Pfeffer MA. Controversies in ventricular remodelling. Lancet 367: 356-367, 2006.

111. Osler W. The Principles and Practice of Medicine. Appleton, New York, NY., 1892.

112. Pyle WG and Solaro RJ. At the crossroads of myocardial signaling: the role of Z-discs in intracellular signaling and cardiac function. Circ Res 94: 296-305, 2004.

113. Rademakers FE and Bogaert J. Left ventricular myocardial tagging. Int J Card Imaging 13: 233-245, 1997.

114. Riedinger F. Uber Brusterschutterung. In: Festschrift zur dritten Saecularfeier der Alam Julia Maximiliana Leipzig. Verlag von F.C.W. Vogel: Leipzig. . 221-234, 1882.

115. Ross J, Jr. Adaptations of the left ventricle to chronic volume overload. Circ Res 35: suppl II:64-70, 1974.

116. Ross RS. Molecular and mechanical synergy: cross-talk between integrins and growth factor receptors. Cardiovasc Res 63: 381-390, 2004.

117. Ross RS, Pham C, Shai SY, Goldhaber Jl, Fenczik C, Glembotski CC, Ginsberg MH, and Loftus JC. Beta1 integrins participate in the hypertrophic response of rat ventricular myocytes. Circ Res 82: 1160-1172, 1998.

118. Ruwhof $\mathrm{C}$ and van der Laarse $\mathrm{A}$. Mechanical stress-induced cardiac hypertrophy: mechanisms and signal transduction pathways. Cardiovasc Res 47: 23-37, 2000.

119. Sachs F and Morris CE. Mechanosensitive ion channels in nonspecialized cells. Rev Physiol Biochem Pharmacol 132: 1-77, 1998.

120. Sachs F, Sigurdson W, and Ruknudin A. Single-channel mechanosensitive currents. Science 253: 800-801, 1991.

121. Sackin H. Mechanosensitive channels. Annu Rev Physiol 57: 333-353, 1995.

122. Sackin H. Stretch-activated ion channels. Kidney Int 48: 1134-1147, 1995.

123. Sadoshima $\mathrm{J}$ and Izumo $\mathrm{S}$. The cellular and molecular response of cardiac myocytes to mechanical stress. Annu Rev Physiol 59: 551-571, 1997.

124. Sadoshima J and Izumo $\mathrm{S}$. Mechanical stretch rapidly activates multiple signal transduction pathways in cardiac myocytes: potential involvement of an autocrine/paracrine mechanism. Embo J 12: 1681-1692, 1993.

125. Sadoshima J, Jahn L, Takahashi T, Kulik TJ, and Izumo S. Molecular characterization of the stretchinduced adaptation of cultured cardiac cells. An in vitro model of load-induced cardiac hypertrophy. $J$ Biol Chem 267: 10551-10560, 1992.

126. Sadoshima J, Takahashi T, Jahn L, and Izumo S. Roles of mechano-sensitive ion channels, cytoskeleton, and contractile activity in stretch-induced immediate-early gene expression and hypertrophy of cardiac myocytes. Proc Natl Acad Sci U S A 89: 9905-9909, 1992.

127. Sadoshima J, Xu Y, Slayter HS, and Izumo S. Autocrine release of angiotensin II mediates stretch-induced hypertrophy of cardiac myocytes in vitro. Cell 75: 977-984, 1993. 
128. Sandler H and Dodge HT. Left Ventricular Tension and Stress in Man. Circ Res 13: 91-104, 1963.

129. Schaub MC, Hefti MA, Harder BA, and Eppenberger HM. Various hypertrophic stimuli induce distinct phenotypes in cardiomyocytes. J Mol Med 75: 901-920, 1997.

130. Schluter KD and Wollert KC. Synchronization and integration of multiple hypertrophic pathways in the heart. Cardiovasc Res 63: 367-372, 2004.

131. Schott E. Uber Ventrikelstillstand (Adams-Stokes'sche Anfalle) nebst Bemerkungen uber andersartige Arhythmien passagerer Natur. Deutsches Archiv fur Klinische medizin 131: 211-229, 1920.

132. Simpson DG, Majeski M, Borg TK, and Terracio L. Regulation of cardiac myocyte protein turnover and myofibrillar structure in vitro by specific directions of stretch. Circ Res 85: e59-69, 1999.

133. Starling EH. The Linacre Lecture on the Law of the Heart. Longmans Green \& Co., London. 1918.

134. Sugden PH and Clerk A. Cellular mechanisms of cardiac hypertrophy. J Mol Med 76: 725-746, 1998.

135. Sugden PH and Clerk A. "Stress-responsive" mitogen-activated protein kinases (c-Jun N-terminal kinases and p38 mitogen-activated protein kinases) in the myocardium. Circ Res 83: 345-352, 1998.

136. Sun M, Chen M, Dawood F, Zurawska U, Li JY, Parker T, Kassiri Z, Kirshenbaum LA, Arnold M, Khokha R, and Liu PP. Tumor necrosis factor-alpha mediates cardiac remodeling and ventricular dysfunction after pressure overload state. Circulation 115: 1398-1407, 2007.

137. Sussman MA, McCulloch A, and Borg TK. Dance band on the Titanic: biomechanical signaling in cardiac hypertrophy. Circ Res 91: 888-898, 2002.

138. Swynghedauw B. Molecular mechanisms of myocardial remodeling. Physiol Rev 79: 215-262, 1999.

139. Swynghedauw B. Remodelling of the heart in response to chronic mechanical overload. Eur Heart J 10: 935-943, 1989.

140. Swynghedauw B and Delcayre C. Biology of cardiac overload. Pathobiol Annu 12: 137-183, 1982.

141. Tomaselli GF and Marban E. Electrophysiological remodeling in hypertrophy and heart failure. Cardiovasc Res 42: 270-283, 1999.

142. Tomaselli GF and Rose J. Molecular aspects of arrhythmias associated with cardiomyopathies. Curr Opin Cardiol 15: 202-208, 2000.

143. Tomaselli GF and Zipes DP. What causes sudden death in heart failure? Circ Res 95: 754-763, 2004.

144. Urheim S, Edvardsen T, Torp H, Angelsen B, and Smiseth OA. Myocardial strain by Doppler echocardiography. Validation of a new method to quantify regional myocardial function. Circulation 102: 1158-1164, 2000.

145. Vakili BA, Okin PM, and Devereux RB. Prognostic implications of left ventricular hypertrophy. Am Heart J 141: 334-341, 2001.

146. Vandenburgh HH, Solerssi R, Shansky J, Adams JW, Henderson SA, and Lemaire J. Response of neonatal rat cardiomyocytes to repetitive mechanical stimulation in vitro. Ann N Y Acad Sci 752: 19-29, 1995.

147. Verdecchia P, Schillaci G, Borgioni C, Ciucci A, Gattobigio R, Zampi I, Santucci A, Santucci C, Reboldi G, and Porcellati $C$. Prognostic value of left ventricular mass and geometry in systemic hypertension with left ventricular hypertrophy. Am J Cardiol 78: 197-202, 1996. 
148. Weber KT, Pick R, Jalil JE, Janicki JS, and Carroll EP. Patterns of myocardial fibrosis. J Mol Cell Cardio/ 21 Suppl 5: 121-131, 1989.

149. Wiggers CJ. The Pressure Pulses in the Cardiovascular System. Longmans Green \& Co. London., 1928.

150. Wright WC. Lancisi's Aneurysms. Macmillan, New York, 1952.

151. Yamamoto K, Dang QN, Maeda Y, Huang H, Kelly RA, and Lee RT. Regulation of cardiomyocyte mechanotransduction by the cardiac cycle. Circulation 103: 1459-1464, 2001.

152. Zerhouni EA, Parish DM, Rogers WJ, Yang A, and Shapiro EP. Human heart: tagging with MR imaging--a method for noninvasive assessment of myocardial motion. Radiology 169: 59-63, 1988.

153. Zingman LV, Alekseev AE, Zingman-Hodgson DM, and Terzic A. ATP-sensitive Potassium Channels: Metabolic Sensing and Cardioprotection. J Appl Physiol, 2007.

154. Zipes DP and Wellens HJ. Sudden cardiac death. Circulation 98: 2334-2351, 1998. 
Dirk W. Donker, Harry J.G.M. Crijns, Paul G.A. Volders.

Cardiac Mechano-Electric Feedback and Arrhythmias: From Pipette to Patient. 2005; Saunders - Elsevier. Peter Kohl, Frederick Sachs, Michael R. Franz (Eds). 


\section{Electro-mechanical remodeling in hypertrophy}




\begin{abstract}
This chapter describes clinical and experimental aspects of electro-mechanical remodeling during long-term hemodynamic overload leading to hypertrophy. The putative role of mechanical stimuli to induce molecular-electrophysiologic changes is discussed. The chapter focuses primarily on the remodeling of ventricular repolarization and its relation to arrhythmogenesis. First, we provide a brief overview of ventricular arrhythmias and sudden cardiac death (SCD) in the general population emphasizing how electrophysiologic changes in the setting of chronic hemodynamic overload can adversely influence these deleterious events.
\end{abstract}




\section{CLINICAL IMPACT OF VENTRICULAR ARRHYTHMIAS AND SUDDEN CARDIAC DEATH}

SCD is responsible for 300,000 to 400,000 victims per year in the United States [1]. In the Maastricht area in The Netherlands, with 182,000 inhabitants, the yearly incidence of out-of-hospital sudden cardiac arrest (death rate, 93\%) at the age of 20 to 75 years is approximately 1 per 1000 people [2]. More than $90 \%$ of these fatalities occur in patients with coronary artery disease or cardiomyopathies. In the remaining $5 \%$ to $10 \%$, no evidence of structural heart disease can be found.

Ventricular arrhythmias are the most frequent cause of sudden death. Often, they are the first manifestation of cardiovascular disease. In a study of patients with sudden arrhythmic death (documented on an ambulatory electrocardiographic [ECG] recorder), ventricular tachyarrhythmias were the terminal event in $83 \%$ of the cases, whereas bradyarrhythmias occurred in 17\% [3]. Three forms of tachyarrhythmia were distinguished: (1) primary ventricular fibrillation (10\% of the $83 \%)$; (2) monomorphic ventricular tachycardia or flutter precipitating ventricular fibrillation (75\%); and (3) polymorphic ventricular tachycardia, including torsades de pointes (15\%) [3]. An example of the latter, from our own registry, is shown in Figure 1. Fortunately, most often ventricular arrhythmias have less detrimental consequences.

Patients with chronic pathologic overload of the heart (e.g., caused by hypertension, valvular disease, chronic ischemia, and infarction) are at increased risk for fatal arrhythmias $[4,5]$ and prone to develop overt heart failure $[6,7]$. There is an increase in the frequency and complexity of ventricular ectopy with the progression of heart failure [8]. Total mortality correlates with left ventricular (LV) function and the presence of complex ventricular ectopy [9]. However, a clear correlation between SCD and LV contractile function or ventricular ectopy has not been found. In fact, data from the Vasodilator Heart Failure Trial (Val-HeFT) Study [10] and the Metoprolol Randomised Intervention Trial in Congestive Heart Failure (MERIT-HF) Trial [11] suggest that death is disproportionately sudden in patients with more modest myocardial dysfunction.

Figure 1 shows an example of sudden arrhythmic death in a patient with compensated ventricular hypertrophy. The recordings are from an 81-year-old woman with a long-term history of severe aortic stenosis, moderate aortic incompetence, and hypertension leading to concentric LV hypertrophy. Aortic valve replacement was strongly recommended but was refused by the patient. Her last medication comprised enalapril, furosemide, carbamazepine, and venlafaxine. She had been in a vital condition with no clinical signs of circulatory decompensation and a normal ejection fraction of $63 \%$ just days before the fatal event.

Pathologic ventricular hypertrophy has long been recognized as an independent risk factor for SCD $[4,6]$. However, the mechanisms of proarrhythmia in conditions with chronic hemodynamic overload are complex. Even in patients with the same pathology, different mechanisms of tachyarrhythmia prevail. In our patient, the presence of a myocardial substrate with electrical and structural altera- 

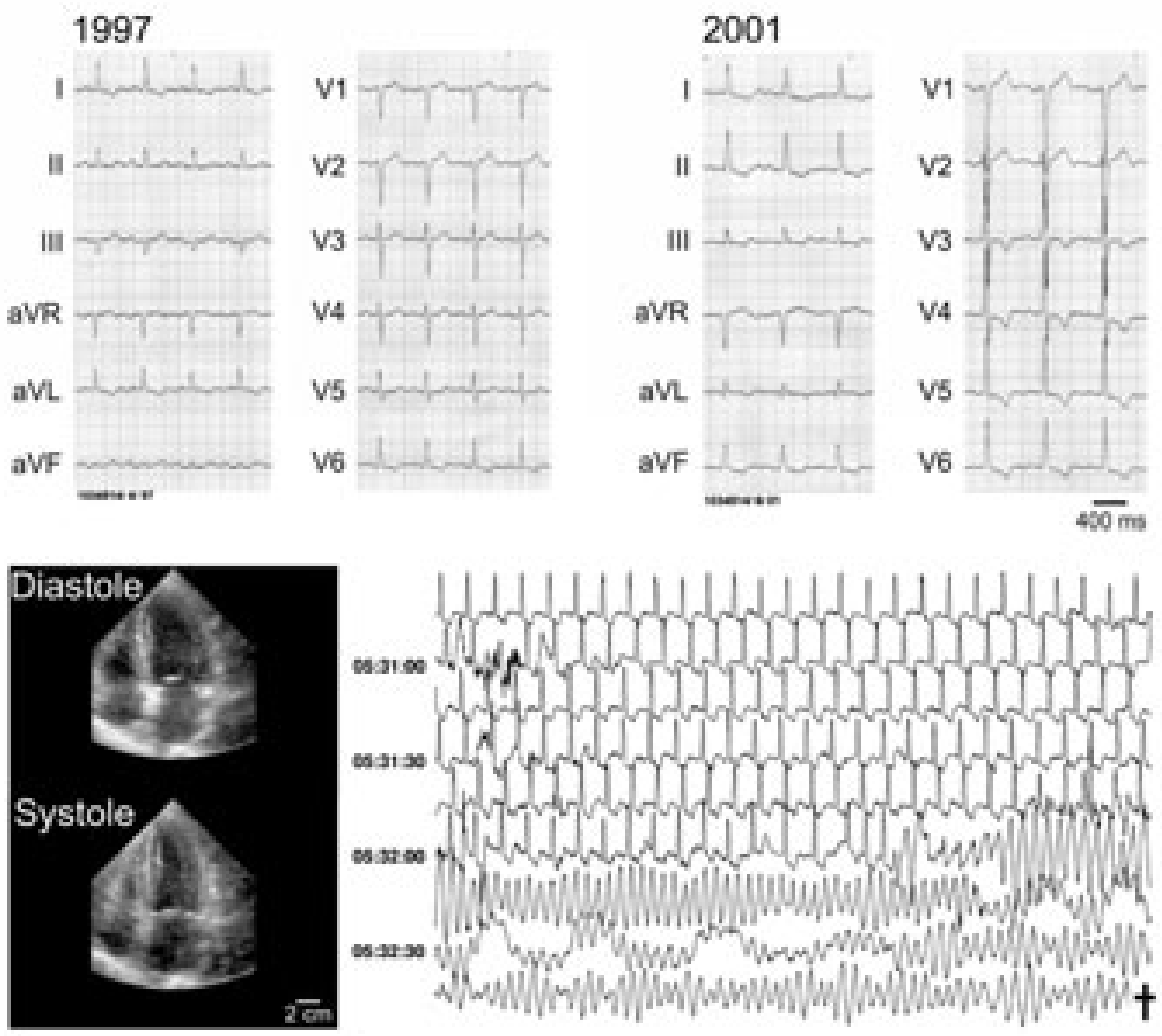

Figure 1 | Sudden arrhythmic death in a patient with compensated ventricular hypertrophy. Top, Twelve-lead electrocardiograms (ECG) in 1997 and 2001 (weeks before death), showing sinus rhythm with normal QT intervals. From 1997 to 2001, the electrical axis changed from horizontal to intermediate, whereas high voltages in the precordial leads and a strain pattern of the ST-T segments developed, compatible with significant hypertrophy. Bottom left, Echocardiogram recorded a few days before the fatal event. Cardiac contractile performance was overall good. Bottom right, Ambulatory ECG showing the sudden onset of polymorphic ventricular tachycardia degenerating into ventricular fibrillation at 05:32 AM. (Courtesy of Joep L.R.M. Smeets, MD, PhD, Department of Cardiology, Academic Hospital Maastricht, Netherlands)

tions (e.g., fibrosis) must be assumed (based on the recordings shown and literature data). The generation of ventricular ectopic beats, triggering the tachycardia, indicated an arrhythmogenic milieu that was possibly influenced by myocardial ischemia, autonomic dysbalance, local conduction disturbances, and/or the side effects of venlafaxine (with a known proarrhythmic risk; for more information, see the University of Arizona Health Sciences Center Web site at: www.torsades.org) 
and furosemide (with a risk for hypokalemia). This case exemplifies the importance of a substrate and triggers for the occurrence of polymorphic ventricular tachycardia in hypertrophy.

\section{ELECTRICAL REMODELING IN CHRONIC CARDIAC OVERLOAD}

\section{General remarks}

The poor understanding of arrhythmias in cardiac hypertrophy and failure has instigated numerous studies, both experimental and clinical. A common finding is the presence of structural and electrical reconstitution of the overloaded myocardium. Many of the molecular safeguards of electrical stability undergo functional or expressional alterations, or both, often by genetic reprogramming. Collectively, these alterations are termed electrical remodeling.

Electrical remodeling is a key characteristic of normal development, expressing functional adaptation to increasing hemodynamic demands during cardiac growth (e.g., see Reference 12). In congenital and acquired heart disease, when hemodynamic load exceeds physiologic limits, the plasticity of the (mal)adaptive response systems determining electrical stability is challenged. The growing list of molecules affected in such conditions comprises ion channels and transporters, components of the $\mathrm{Ca}^{2+}$-handling machinery, and structural proteins of the myocytes and interstitium [13]. Importantly, their alterations may already be significant in an early stage of overload, when contractility is still adequate to maintain cardiac output at (near-)normal levels. This might explain, at least in part, why a relatively high proportion of patients with compensated or mildly decompensated myocardial function is susceptible to arrhythmias and SCD.

A hallmark of electrical remodeling in hypertrophy and failure, independent of the cause, is prolongation of the ventricular action potential (for excellent reviews, refer to Tomaselli and coworkers [5, 13]). For the human heart, action potential prolongation occurs both in compensated hypertrophy [14] and in terminal heart failure $[15,16]$. In the three-dimensional structure of the heart, regional differences in action potential duration (across the ventricular wall, between the ventricles, and from base to apex) may exaggerate during hypertrophy $[17,18]$ and failure, $[19,20]$ and predispose to ventricular arrhythmias. Conversely, a reduction in electrophysiologic gradients may also be proarrhythmic (see Chapter 22)'. Changes in repolarization may present as perturbations of the normal epicardial to endocardial direction of recovery from electrical activation, which can turn arrhythmogenic [21]. OT-interval labilities in patients with ischemic and nonischemic dilated cardiomyopathy, [22] and other disorders with hypertrophy or failure, are another indication that the substrate for arrhythmias is related, at least in part, to impaired repolarization. Importantly, the

1 Cardiac Mechano-Electric Feedback and Arrhythmias: From Pipette to Patient. 2005 
presence of severe repolarization abnormalities can be hidden under a "normal" action potential morphology and duration, or a "normal" OT interval in the surface ECG. In such conditions, a modest challenge of electrical stability can evoke profound proarrhythmic responses.

Repolarization lability in cardiac hypertrophy and failure is commonly based on $\mathrm{K}^{+}$-channel downregulation $[23,24]$ and altered $\mathrm{Ca}^{2+}$ homeostasis [25]. These changes may have a gene-transcriptional basis, $[26,27]$ although some reports indicate the additional importance of post-transcriptional alterations [28]. Information about the exact time course of electrical changes and underlying molecular mechanisms is scarce. Also, the identity of mechanical or biochemical stimuli that trigger electrical remodeling is largely unknown.

\section{Proarrhythmia in compensated ventricular hypertrophy: the dog model with chronic complete atrioventricular block}

A dog model of volume overload caused by complete atrioventricular block (AVB) has been extensively studied. Important electrical, contractile, and structural alterations of the myocardium are found in this model $[18,29]$. Myocardial adaptation is associated with an enhanced susceptibility to torsades de pointes $[18,29]$ and SCD. Most dogs exhibit compensated contractile function in the chronic stage of AVB (CAVB). Figure 2 illustrates the key features of this model as currently known. In vivo electrophysiologic recordings reveal significant QT prolongation with broad-based T waves in CAVB. Monophasic action potential recordings from the endocardium indicate that repolarization prolongation is more prominent in the LV than the right ventricle (RV), leading to exaggerated interventricular dispersion of repolarization. Torsades de pointes occur often spontaneously or can be readily induced by programmed electrical stimulation, repolarization-prolonging drugs, or both, which makes this animal model suitable for chronic studies of proarrhythmia.

At the cellular level, significant down-regulation of the $\mathrm{K}^{+}$currents $\mathrm{I}_{\mathrm{Ks}}$ and $\mathrm{I}_{\mathrm{Kr}}$ [24], in combination with enhanced $\mathrm{Na}^{+}-\mathrm{Ca}^{2+}$ exchange [31], contribute to the alteration of spatial and temporal action potential heterogeneities. In addition, enhanced sarcoplasmic reticulum $\mathrm{Ca}^{2+}$ release [31] and an increased $\mathrm{Na}^{+}$concentration [32], together with enhanced $\mathrm{Na}^{+}-\mathrm{Ca}^{2+}$ exchange, can cause $\mathrm{Ca}^{2+}$ dependent abnormal impulse formation [33].

Regarding the $\mathrm{K}^{+}$channel down-regulation, important information comes from a recent study of KCNO1 and KCNE1, the genes encoding for the $\alpha$ and $\beta$ subunit of the $I_{\mathrm{Ks}}$ channel [27]. Results are shown in Figure 3. In CAVB, KCNQ1 complementary DNA (cDNA; reverse-transcribed from messenger RNA) decreases by $80 \%$ and $90 \%$ in the LV and RV, respectively. KCNE1 cDNA diminishes by $70 \%$ and $75 \%$. KCNQ1 protein expression in CAVB is decreased by $60 \%$ and $45 \%$ in the LV and RV, whereas the expression of KCNE1 decreases by $60 \%$ and $70 \%$, respectively. The earlier finding of a $50 \%(\mathrm{LV})$ and $55 \%(\mathrm{RV})$ reduction in $\mathrm{I}_{\mathrm{Ks}}$ density [24] is in good agreement with this molecular data. Taken together, these findings (and additional results not described here [27]) indicate that 


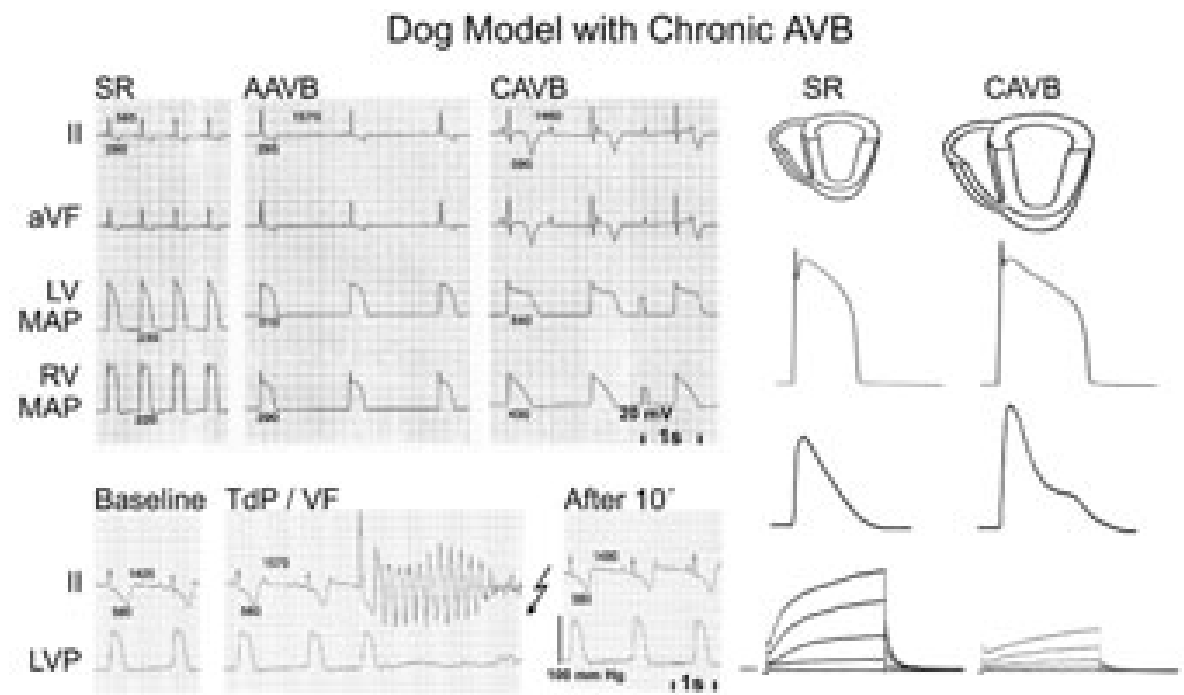

Figure 2 | Dog model with chronic atrioventricular block (CAVB). Top left, Acquired QT prolongation. ECG leads II and aVF and simultaneous monophasic action potential recordings from the endocardium of the left (LV MAP) and right ventricle (RV MAP). Bottom left, Spontaneous torsades de pointes (TdP) deteriorating into ventricular fibrillation (VF) with hemodynamic collapse. ECG lead II and LV pressure (LVP) recordings during anesthesia. After successful defibrillation, electrical and hemodynamic stability was regained within minutes. Right, Cellular basis of proarrhythmia in dilative ventricular hypertrophy after CAVB. Transmembrane action potential prolongation and enhanced $\mathrm{Ca}^{2+}$ release from the sarcoplasmic reticulum (second and third row from the top, respectively) are typical features. Enhanced $\mathrm{Na}^{+}-\mathrm{Ca}^{2+}-$ exchange activity and down-regulation of the delayed-rectifier $\mathrm{K}^{+}$current $_{\mathrm{Ks}}$ (bottom traces) contribute to action potential prolongation. SR, sinus rhythm; AAVB, Acute atrioventricular block. (Top left, Reproduced from Volders PGA, Sipido KR, Vos MA, et al: Downregulation of delayed rectifier $\mathrm{K}^{+}$currents in dogs with chronic complete atrioventricular block and acquired torsades de pointes. Circulation 100:24552461, 1999, by permission of the American Heart Association; bottom left, acknowledgment to Jet D. M. Beekman, Department of Medical Physiology, University Medical Center Utrecht, Netherlands.). 

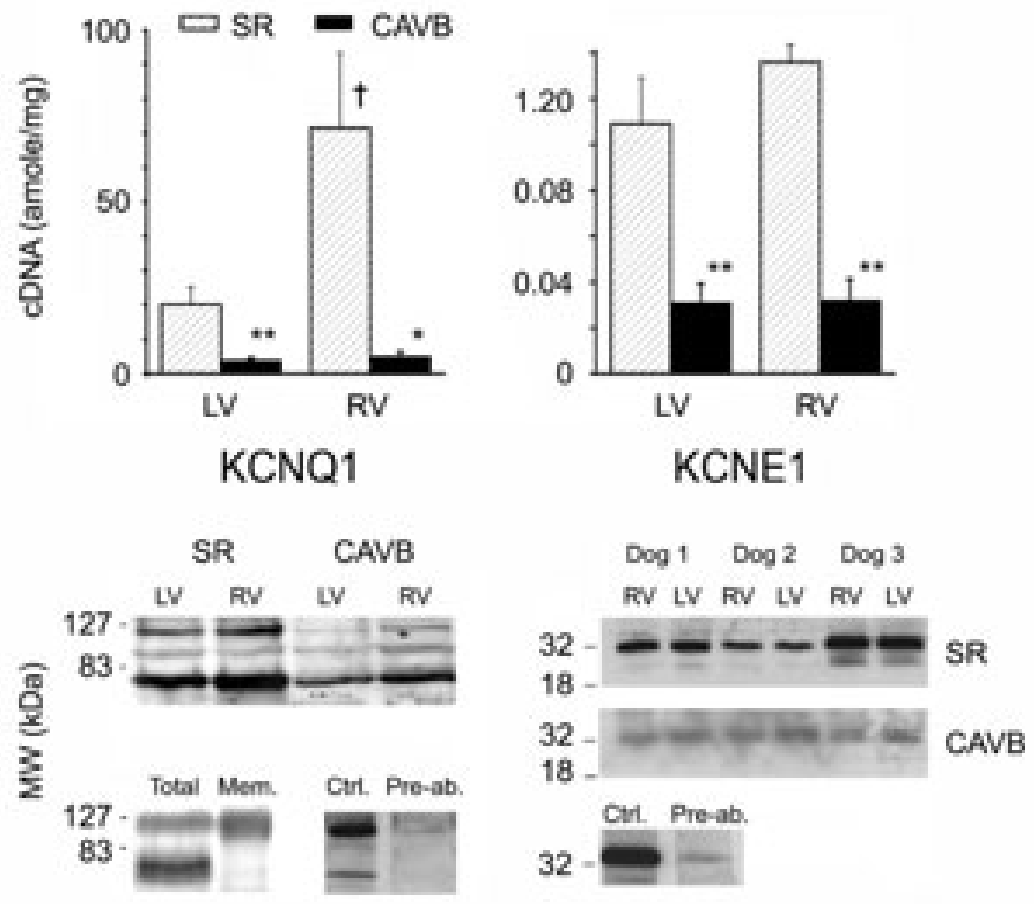

Figure $3 \mid$ Reduction of $\mathrm{I}_{\mathrm{Ks}}$ in chronic atrioventricular block (CAVB) is caused by down-regulation of KCNQ1 and KCNE1 mRNA and protein expression. Top, Competitive multiplex reverse transcription polymerase chain reaction (PCR) showed CAVB-induced reduction of KCNQ1 and KCNE1 complementary DNA (messenger RNA). Middle left, Western blot of myocardial proteins from left ventricle (LV) and right ventricle (RV) of a sinus rhythm (SR) and CAVB dog. Probing with KCNQ1 polyclonal antibody identified two bands with molecular weights (MW) of $\sim 72$ and $\sim 120 \mathrm{kDa}$ at SR. Bottom left, Experiments repeated on total proteins (Total) and membrane proteins (Mem.) isolated from LV cardiac myocytes at SR. Both 72- and 120-kDa bands were recognized in the total protein preparation, but only the 120-kDa band was predominant in proteins isolated from the membrane fraction. At the right, to verify that both bands were linked to KCNQ1, immunoblots were performed using preabsorbed (Pre-ab.) KCNQ1 antibodies. Middle right, Western blot of proteins extracted from the LV and RV of SR and CAVB dogs (Dogs 1 to 3) and probed with KCNE1 polyclonal antibody. The KCNE1 antibody recognized a band $\sim 35 \mathrm{kDa}$ and a faint band $\sim 20 \mathrm{kDa}$ in SR. In both blots (left and right), densitometric analysis revealed significant down-regulation of KCNQ1 and KCNE1 proteins during CAVB in both ventricles. Bottom right, Control Western blots using proteins probed with anti-KCNE1 antibodies (Ctrl.) or with antibodies preabsorbed against the KCNE1 antigen (Pre-ab.), showing that the 35-KDa antigen contained the KCNE1-specific epitope. ${ }^{*} \mathrm{P}<0.05$; ${ }^{* *} \mathrm{P}<0.01$ (SR vs. CAVB); $\uparrow \mathrm{P}<0.05$ (RV vs. LV). (Modified from Ramakers $\mathrm{C}$, Vos MA, Doevendans $\mathrm{PA}$, et al: Coordinated downregulation of KCNQ1 and KCNE1 expression contributes to reduction of $\mathrm{I}_{\mathrm{Ks}}$ in canine hypertrophied hearts. Cardiovasc Res 57:486-496, 2003, with permission). 
the decrease of $\mathrm{I}_{\mathrm{Ks}}$ involves the regulation of KCNQ1 and KCNE1 gene expression at the transcriptional and translational levels.

\section{MISSING LINKS BETWEEN CHRONIC MECHANICAL OVERLOAD AND ELECTRICAL REMODELING}

In the previous section, we described the molecular down-regulation of KCNQ1 and KCNE1 proteins that coassemble to form the $I_{\mathrm{Ks}}$ channel. For all acquired channelopathies currently known to us, the primary stimuli for down-regulation are unknown. However, mechanical stimuli are obvious candidates.

Recently, we have quantified mechanical overload in the dog model with AVB and found that diastolic stress, diastolic strain (i.e., stretch), systolic strain, and stroke work are all significantly increased at the myocardial level [34]. The imposition of bradycardia-induced volume overload is followed by the development of ventricular hypertrophy and QT prolongation. Whether and how any (or all) of the mechanical changes could influence $\mathrm{I}_{\mathrm{Ks}}$ channel expression through transcriptional or translational regulation, or both (or other molecular mechanisms), is an intriguing question.

In contemporary working models of mechanosensing and -transduction by the cardiac myocyte, a major role is attributed to a protein complex of integrin $\beta_{10} /$ talin/melusin at the sarcolemma/Z-disc, whose importance in pressure overload has been demonstrated [35]. In addition, some cardiac mechanical stretch sensor machinery incorporates a protein complex of titin, muscle LIM protein (MLP), and telethonin (T-cap) interacting at the Z-disc (Figure 4A) [36]. Defects in the MLP/T-cap complex lead to dilative cardiomyopathy in MLP-/- transgenic mice and a subset of human dilative cardiomyopathy [36]. Furukawa and colleagues [37] have described that the extreme C-terminal region of T-cap binds to the cytoplasmic domain of KCNE1 (=minK; see Figure 4B). It is attractive to speculate that this molecular chain of titin/MLP/T-cap/KCNE1 serves as conduit for mechanoelectric remodeling. Whether and how the findings of gene-transcriptional and translational alterations [27] fit into this hypothetical scheme remains to be determined.

Mechanical influences could also alter transcriptional activity through the intermediate filament protein desmin. Bloom and colleagues [38] have shown that changes in the spatial arrangement of both the desmin-lamin intermediate filament network and the nuclear envelope-associated chromatin can be induced by mechanical stretch. We have recently found that desmin expression decreases early after the induction of AVB in dogs [39], parallel to $\mathrm{I}_{\mathrm{KS}}$ down-regulation, which could also indicate a mechano-electric link.

More research is needed to answer these important questions of mechano-electric coupling during chronic overload. 


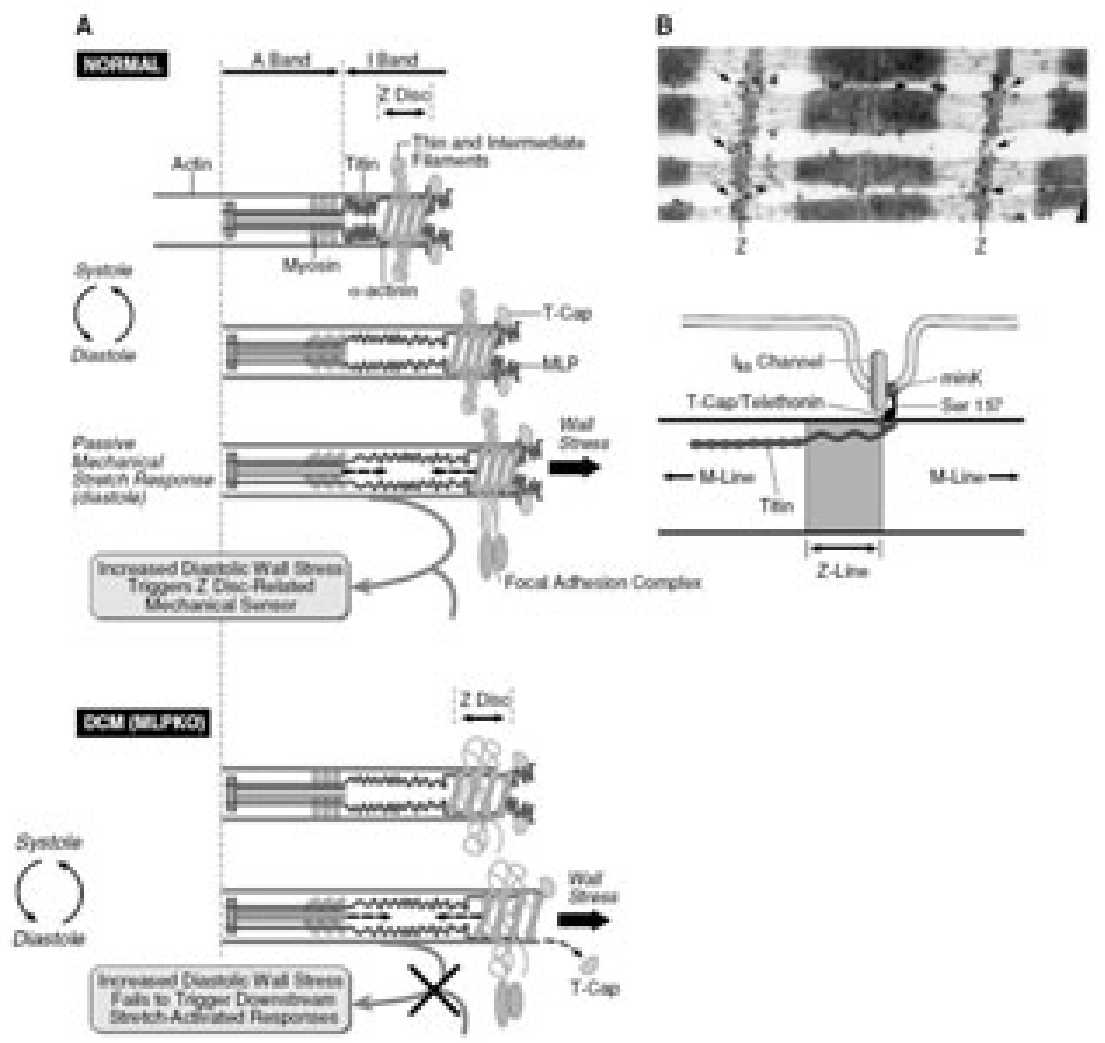

Figure $4 \mid$ A Proposed working model of the cardiac stretch sensor machinery. Top, Normal conditions. Titin, anchored between Z-disc and M-line, exhibits elastic "molecular springs" (I band). Titin is able to store force generated by systolic compression (systole), and it largely determines passive myocardial stiffness (diastole). With increasing diastolic wall stress, the titin/MLP/telethonin (T-cap) complex is stretched and mechanical load is sensed. Bottom, Loss of the MLP/T-cap complex in knockout mice (MLPKO) causes a defective stretch-sensor function, ultimately leading to dilative cardiomyopathy (DCM), which, in turn, enhances wall stress and mechanical stretch stimuli. B, Model for the titin/T-cap/minK complex in cardiac myocytes. Top, Detection of T-cap by immunoelectron microscopy in skeletal muscle. T-cap is clustered where membrane structures corresponding to T-tubules and terminal cisternae contact the Z-line periphery (arrows). Bottom, Summary of known interactions of titin, T-cap, and minK (=KCNE1). T-cap connects the myofibrillar apparatus to the sarcolemma at the Z-line periphery. Phosphorylation of the C-terminal tail of the T-cap by yet unidentified kinases may regulate the stability of the complex. (A, Reproduced from Knöll R, Hoshijima M, Hoffman HM, et al: The cardiac mechanical stretch sensor machinery involves a Z disc complex that is defective in a subset of human dilated cardiomyopathy. Cell 111:943-955, 2002, with permission; B, reproduced from Furukawa T, Ono Y, Tsuchiya $\mathrm{H}$, et al: Specific interaction of the potassium channel $\beta$-subunit minK with the sarcomeric protein T-cap suggests a T-tubule-myofibril linking system. J Mol Biol 313:775-784, 2001, with permission.). 


\section{Reversibility of electrical remodeling by mechanical unloading?}

New insights in the role of mechanical overload derive from studies of cardiac unloading. Different strategies have been successfully proven to decrease LV hypertrophy and improve cardiac contractility after relief from chronically increased mechanical load [40]. Thus, contractile remodeling is potentially reversible, which could also apply to electrical remodeling. Patients treated for hypertension with enalapril [41] and losartan [42] exhibited decreased rate-corrected QT intervals. Several experimental studies showed related findings $[43,44]$. It is conceivable that the treatment with drugs affects signaling cascades independent of a reduction of mechanical load. However, pure mechanical unloading can also lead to a normalization of altered electrical properties $[45,46]$. This underscores the importance of mechano-transduction for the reversal of electrical remodeling during unloading, even after months and years of overload.

In contrast, other studies have reported that reverse electrical remodeling is absent during drug treatment [47], surgical intervention [48], or ventricular pacing (in CAVB) [49]. These issues clearly are not settled (see Chapters 34 to 37$)^{2}$. Further investigations are warranted.

\section{Electro-mechanical remodeling}

It can be concluded from the previous sections that chronic mechanical overload might well instigate and modulate electrical remodeling, and vice versa, that altered electrical properties of hypertrophied cardiac myocytes can also influence contraction, and thereby myocardial mechanics.

In rodents, action potential prolongation may set the stage for increased $\mathrm{Ca}^{2+}$ transients [50]. thus enhancing contraction and systolic mechanical load. Sah and colleagues [51] have shown that membrane repolarization by the transient outward current, $I_{\text {to }}$, modulates the recruitment and synchronization of sarcoplasmic reticulum $\mathrm{Ca}^{2+}$ release through the L-type $\mathrm{Ca}^{2+}$ current. Interestingly, the same group demonstrated in quiescent neonatal rat ventricular myocytes that a reduction of Kv4.2/3 (responsible for $I_{\text {to }}$ ) is followed by hypertrophy, with a proposed causal role for $\mathrm{Ca}^{2+}$-dependent activation of calcineurin [52]. Although the causality has been questioned [53], these data suggest that the relation between altered electrical properties and cellular hypertrophy may not always incorporate mechanical stimuli.

Based on studies with the $\mathrm{Ca}^{2+}$ indicators aequorin [54] and Indo-1 [55], $\mathrm{Ca}^{2+}$-dependent diastolic dysfunction may also directly influence diastolic mechanics.

In the canine model with CAVB, yet another mechanism of electro-mechanical remodeling applies. Action potential prolongation and altered cellular $\mathrm{Ca}^{2+}$ and $\mathrm{Na}^{+}$handling contribute to enhanced contractile performance, but also to proarrhythmia [31]. Systolic strain, representing myofiber deformation during contraction, was significantly increased in AVB, in addition to a greater diastolic mechanical load [34]. This can be interpreted as a direct mechanical consequence of the described electrical alterations.

2 Cardiac Mechano-Electric Feedback and Arrhythmias: From Pipette to Patient. 2005 
Taken together, the available data suggest that chronic mechano-electric feedback forms a closedloop system with electro-mechanical remodeling, which was presented in detail in the introductory chapters of this book (see Preface) ${ }^{3}$.

\section{NEED FOR SERIAL IN VIVO ASSESSMENT OF MYOCARDIAL MECHANICS DURING CHRONIC OVERLOAD}

Quantification of the mechanical load imposed on the myocardium during chronic overload could offer mechanistic insights and improve clinical decision making. Recent techniques of tagged magnetic resonance imaging [56] and ultrasound strain-rate imaging [57] may provide this information. We have developed an alternative method to quantify myocardial mechanics, combining trans thoracic echocardiography, invasive LV pressure recordings, and mathematical modeling (Figure 5) $[34,58]$. This method is feasible in anesthetized experimental animals and also in patients. Myofiber stress $\left(\sigma_{\mathrm{f}} ; \mathrm{kPa}\right)$ is defined as mechanical force per myofiber cross-sectional area and is calculated as:

$$
\sigma_{f}=L V P\left(\frac{1+3 V_{L V}}{V_{W}}\right)
$$

with $V_{L V}$ and $V_{W}$ representing LV cavity and wall volume. Natural myofiber strain ( $e_{f} ;$ dimensionless), representing deformation, is defined as the natural logarithm of myofiber length (I) relative to its reference length $\left(\mathrm{I}_{\text {ref }}\right)$ at beginning of ejection during sinus rhythm:

$$
e_{f}=\operatorname{In}\left(\frac{I}{I_{r e f}}\right)=\frac{1}{3} \operatorname{In}\left(\frac{1 / 3 V_{W}+V_{L V}}{1 / 3 V_{W, r e f}+V_{L V, r e f}}\right)
$$

Myofiber stroke work $\left(\mathrm{w}_{\mathrm{f}} ; \mathrm{kJ} / \mathrm{m}^{3} /\right.$ beat $)$ is computed as the area of the $\sigma_{\mathrm{f}}-\mathrm{e}_{\mathrm{f}}$ loop, a local analog of the LV pressure-volume loop:

$$
w_{f}=\int_{\text {cycle }} \sigma_{f} d e_{f}
$$

3 Cardiac Mechano-Electric Feedback and Arrhythmias: From Pipette to Patient. 2005 


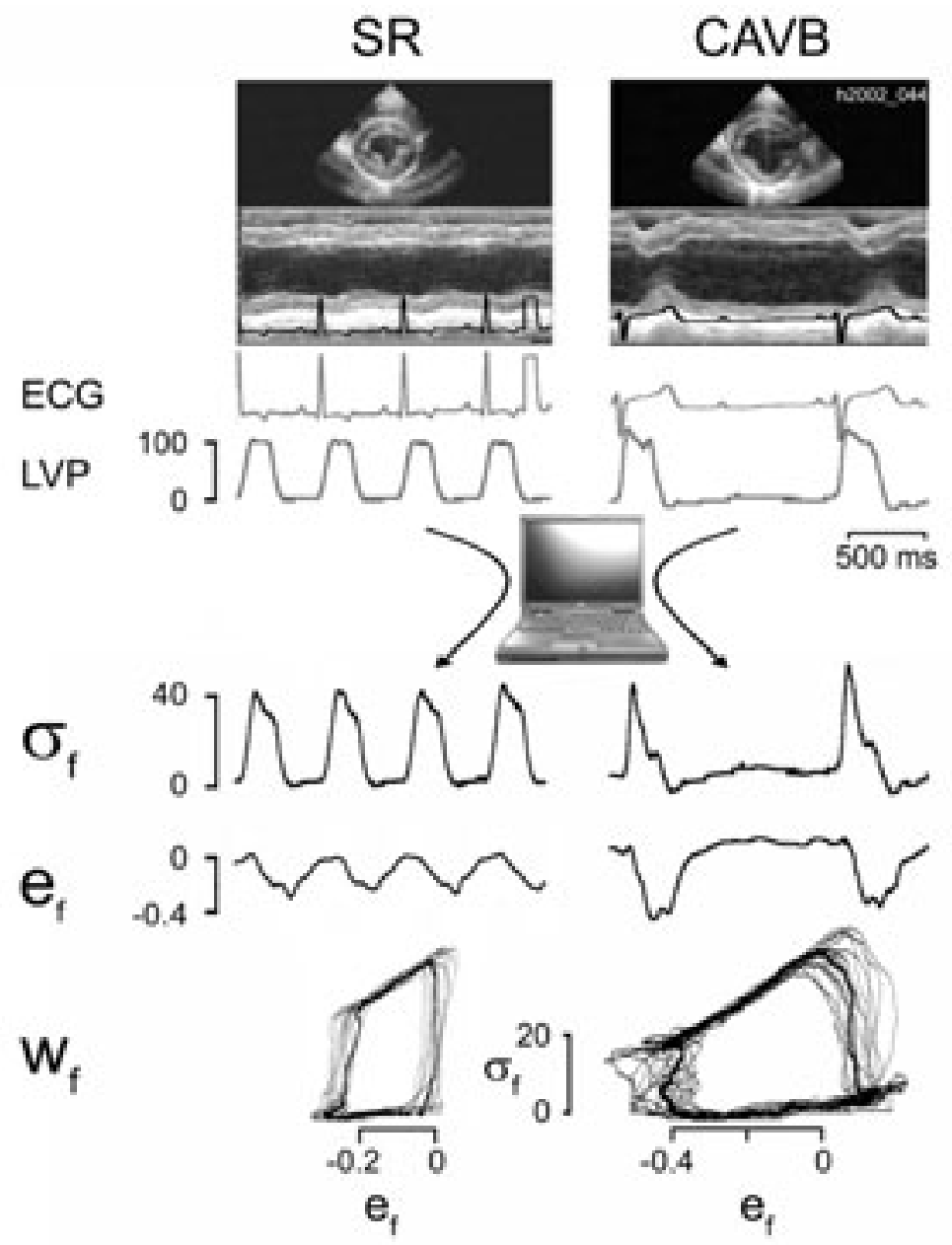

Figure $\mathbf{5}$ |Serial quantification of myofiber mechanics throughout the cardiac cycle during chronic ventricular overload. Anesthetized dogs, before (sinus rhythm [SR]) and in chronic atrioventricular block (CAVB; 6 weeks). Top to bottom, Echographic M-mode (left ventricular [LV] mid-papillary plane). Endocardial and epicardial borders were delineated to calculate LV cavity and wall volume, incorporating longitudinal axes. LV dimensions during the cardiac cycle were electrocardiogram (ECG)-synchronized with LV pressure (LVP). Note time marker on ECG tracing in SR. A validated mathematical model [58] was used to calculate myofiber stress $\left(\sigma_{f}\right)$, natural myofiber strain ( $e_{f}$; dimensionless), and myofiber stroke work $\left(w_{f}\right)$ represented by the area of the $\sigma_{f}-e_{f}$ loop. Note that a negative $e_{f}$ during systole indicates myofiber shortening. A positive $e_{f}$ during diastole designates outward deformation; both occur during CAVB. 
Our approach allows serial mechanical phenotyping during long-term cardiac overload to: (1) characterize the nature and timing of mechanical stimuli throughout the cardiac cycle (see Figure 5); (2) discern the effects of mechanical versus other stimuli of remodeling; (3) define the transition from a functionally compensated to a decompensated state; and (4) evaluate the effects of medical and surgical interventions influencing cardiac overload.

Widespread experimental and clinical application of the aforementioned techniques is awaited and may help to characterize serial mechanical phenotypes in chronic heart disease and their link to electrical and structural remodeling.

\section{CONCLUSIONS}

Mechanical load is chronically increased in common cardiac diseases such as hypertension, valvular heart disease, and myocardial infarction. Ventricular hypertrophy often ensues as an adaptational response, but it is associated with significant morbidity and mortality. Electrical remodeling, notably impaired ventricular repolarization, can pose significant problems. It can manifest as prolongation of the ventricular action potential, but it may also be hidden under a "normal" action potential morphology and duration. The underlying alterations of $\mathrm{K}^{+}$current expression and $\mathrm{Ca}^{2+}$ handling often are based on changes of genetic transcription or post-transcriptional modification.

Currently, the role of pathologic mechanical load to the molecular mechanisms initiating and maintaining electrical remodeling is not understood. Recent findings indicate that myofiber stress or strain, or both, are primary mechanical stimuli sensed by the myocytes through protein complexes of integrin $\beta_{10} /$ talin/melusin at the sarcolemma/Z-disc and/or titin/MLP/T-cap at the Z-disc. A physical connection between the titin/T-cap complex and the $B$ subunit of the $I_{\mathrm{Ks}}$ channel, KCNE1, has been demonstrated and might be involved in the transduction of mechanical overload to $\mathrm{K}^{+}$ channel down-regulation. The translation of these elementary findings to mechano-transduction in the intact heart of humans and animal models requires detailed knowledge of the characteristics of myocardial mechanics during chronic overload. In the dog model with CAVB, increased diastolic stress, diastolic strain, systolic strain, and stroke work are associated with transcriptional changes of the $\mathrm{I}_{\mathrm{Ks}}$ channel subunits KCNO1 and KCNE1. A causal role for the mechanical stimuli is speculative and should be investigated at the molecular-genetic level.

The available data on cardiac electrophysiology and mechanics in chronic overload convince us that, as in acute studies, electro-mechanical signaling forms a closed-loop system with mechanoelectric feedback. Our new approach of calculating mechanics in the intact beating heart may prove useful for understanding how mechano-transduction may lead to remodeling. 
CHAPTER 2 | ELECTRO-MECHANICAL REMODELING IN HYPERTROPHY

\section{Acknowledgments}

Dr. Volders is supported by The Netherlands Organization for Health Research and Development (ZonMw 906-02-068). The authors are grateful to Theo Arts, PhD (Departments of Biophysics, Cardiovascular Research Institute Maastricht and Biomedical Engineering, Eindhoven University of Technology, Netherlands) for his excellent scientific contribution. Roel L.H.M.G. Spätjens, BS (Department of Cardiology, Cardiovascular Research Institute Maastricht) prepared the figures. 


\section{REFERENCES}

1. Zipes DP, Wellens HJJ: Sudden cardiac death. Circulation 98:2334-2351, 1998.

2. de Vreede-Swagemakers JJM, Gorgels APM, Dubois-Arbouw WI, et al: Out-of-hospital cardiac arrest in the 1990's: A population-based study in the Maastricht area on incidence, characteristics and survival. J Am Coll Cardiol 30:1500-1505, 1997.

3. Bayés de Luna A, Coumel P, Leclercq JF: Ambulatory sudden cardiac death: Mechanisms of production of fatal arrhythmia on the basis of data from 157 cases. Am Heart J 117:151-159, 1989.

4. Haider AW, Larson MG, Benjamin EJ, et al: Increased left ventricular mass and hypertrophy are associated with increased risk for sudden death. J Am Coll Cardiol 32:1454-1459, 1998.

5. Tomaselli GF, Marbán E: Electrophysiological remodeling in hypertrophy and heart failure. Cardiovasc Res 42:270-283, 1999

6. Kannel WB, Gordon T, Offutt D: Left ventricular hypertrophy by electrocardiogram. Prevalence, incidence, and mortality in the Framingham study. Ann Intern Med 71:89-105, 1969.

7. Rame JE, Ramilo M, Spencer $N$, et al: Development of a depressed left ventricular ejection fraction in patients with left ventricular hypertrophy and a normal ejection fraction. Am J Cardiol 93:234-237, 2004.

8. Kjekshus J: Arrhythmias and mortality in congestive heart failure. Am J Cardiol 65:42I-48I, 1990.

9. Wilson JR, Schwartz JS, Sutton MS, et al: Prognosis in severe heart failure: Relation to hemodynamic measurements and ventricular ectopic activity. J Am Coll Cardiol 2:403-410, 1983.

10. Cohn JN, Archibald DG, Ziesche S, et al: Effect of vasodilator therapy on mortality in chronic congestive heart failure. Results of a Veterans Administration Cooperative Study. N Engl J Med 314:1547-1552, 1986.

11. Effect of metoprolol CR/XL in chronic heart failure: Metoprolol CR/XL Randomised Intervention Trial in Congestive Heart Failure (MERIT-HF). Lancet 353:2001-2007, 1999.

12. Jeck CD, Boyden PA: Age-related appearance of outward currents may contribute to developmental differences in ventricular repolarization. Circ Res 71:1390-1403, 1992.

13. Armoundas AA, Wu R, Juang G, et al: Electrical and structural remodeling of the failing ventricle. Pharmacol Ther 92:213-230, 2001.

14. Bailly $P$, Bénitah JP, Mouchoniàre $M$, et al: Regional alteration of the transient outward current in human left ventricular septum during compensated hypertrophy. Circulation 96: 1266-1274, 1997.

15. Beuckelmann DJ, Näbauer M, Erdmann E: Alterations of $\mathrm{K}^{+}$currents in isolated human ventricular myocytes from patients with terminal heart failure. Circ Res 73:379-385, 1993.

16. Vermeulen JT, McGuire MA, Opthof T, et al: Triggered activity and automaticity in ventricular trabeculae of failing human and rabbit hearts. Cardiovasc Res 28:1547-1554, 1994.

17. Keung ECH, Aronson RS: Non-uniform electrophysiological properties and electrotonic interaction in hypertrophied rat myocardium. Circ Res 49:150-158, 1981. 
18. Volders PGA, Sipido KR, Vos MA, et al: Cellular basis of biventricular hypertrophy and arrhythmogenesis in dogs with chronic complete atrioventricular block and acquired torsade de pointes. Circulation 98:1136-1147, 1998.

19. Näbauer $M$, Beuckelmann DJ, Überfuhr $P$, et al. Regional differences in current density and ratedependent properties of the transient outward current in subepicardial and subendocardial myocytes of human left ventricle. Circulation 93:168-177, 1996.

20. Akar FG, Rosenbaum DS: Transmural electrophysiological heterogeneities underlying arrhythmogenesis in heart failure. Circ Res 93:638-645, 2003.

21. Fish JM, Di Diego JM, Nesterenko V, et al: Epicardial activation of left ventricular wall prolongs QT interval and transmural dispersion of repolarization: Implications for biventricular pacing. Circulation 109:2136-2142, 2004.

22. Berger RD, Kasper EK, Baughman KL, et al: Beat-to-beat $\mathrm{OT}$ interval variability: Novel evidence for repolarization lability in ischemic and nonischemic dilated cardiomyopathy. Circulation 96:1557-1565, 1997.

23. Näbauer M, Kääb S: Potassium channel down-regulation in heart failure. Cardiovasc Res 37:324-334, 1998.

24. Volders PGA, Sipido KR, Vos MA, et al: Downregulation of delayed rectifier $\mathrm{K}^{+}$currents in dogs with chronic complete atrioventricular block and acquired torsades de pointes. Circulation 100:2455-2461, 1999.

25. Sipido KR, Volders PGA, Vos MA, et al: Altered $\mathrm{Na} / \mathrm{Ca}$ exchange activity in cardiac hypertrophy and heart failure: A new target for therapy? Cardiovasc Res 53:782-805, 2002.

26. Kääb S, Dixon J, Duc J, et al: Molecular basis of transient outward potassium current downregulation in human heart failure: A decrease in Kv4.3 mRNA correlates with a reduction in current density. Circulation 98:1383-1393, 1998.

27. Ramakers C, Vos MA, Doevendans PA, et al: Coordinated down-regulation of KCNQ1 and KCNE1 expression contributes to reduction of IKs in canine hypertrophied hearts. Cardiovasc Res 57:486-496, 2003.

28. Zicha S, Maltsev VA, Nattel S, et al: Post-transcriptional alterations in the expression of cardiac $\mathrm{Na}^{+}$ channel subunits in chronic heart failure. J Mol Cell Cardiol 37:91-100, 2004.

29. Vos MA, de Groot SHM, Verduyn SC, et al: Enhanced susceptibility for acquired torsade de pointes arrhythmias in the dog with chronic, complete AV block is related to cardiac hypertrophy and electrical remodeling. Circulation 98:1125-1135, 1998.

30. van Opstal JM, Verduyn SC, Leunissen HDM, et al: Electrophysiological parameters indicative of sudden cardiac death in the dog with chronic complete AV-block. Cardiovasc Res 50:354-361, 2001.

31. Sipido KR, Volders PGA, de Groot SHM, et al: Enhanced $\mathrm{Ca}^{2+}$ release and $\mathrm{Na} / \mathrm{Ca}$ exchange activity in hypertrophied canine ventricular myocytes: Potential link between contractile adaptation and arrhythmogenesis. Circulation 102:2137-2144, 2000.

32. Verdonck F, Volders PGA, Vos MA, et al: Increased $\mathrm{Na}^{+}$concentration and altered $\mathrm{Na} / \mathrm{K}$ pump activity in hypertrophied canine ventricular cells. Cardiovasc Res 57:1035-1043, 2003.

33. Sipido KR, Volders PGA, Schoenmakers $\mathrm{M}$, et al: Role of the $\mathrm{Na} / \mathrm{Ca}$ exchanger in arrhythmias in compensated hypertrophy. Ann N Y Acad Sci 976:438-445, 2002. 
34. Donker DW, Volders PGA, Borgers M, et al: Increases of systolic fiber strain and end-diastolic fiber stress are the primary local mechanical changes in chronic heart block [abstract]. Eur Heart J 25(suppl): 555, 2004.

35. Brancaccio $M$, Fratta $L$, Notte $A$, et al: Melusin, a muscle-specific integrin B1-interacting protein, is required to prevent cardiac failure in response to chronic pressure overload. Nat Med 9:68-75, 2003.

36. Knöll R, Hoshijima M, Hoffman HM, et al: The cardiac mechanical stretch sensor machinery involves a Z disc complex that is defective in a subset of human dilated cardiomyopathy. Cell 111:943-955, 2002.

37. Furukawa $\mathrm{T}, \mathrm{Ono} \mathrm{Y}$, Tsuchiya $\mathrm{H}$, et al: Specific interaction of the potassium channel B-subunit minK with the sarcomeric protein T-cap suggests a T-tubule-myofibril linking system. J Mol Biol 313:775-784, 2001.

38. Bloom S, Lockard VG, Bloom M: Intermediate filament-mediated stretch-induced changes in chromatin: A hypothesis for growth initiation in cardiac myocytes. J Mol Cell Cardiol 28:2123-2127, 1996.

39. Donker DW, Volders PGA, Maessen JG, et al: Time frame of myocardial dedifferentiation in canine ventricular hypertrophy [abstract]. J Mol Cell Cardiol 34:A20, 2002.

40. Zafeiridis A, Jeevanandam V, Houser SR, et al: Regression of cellular hypertrophy after left ventricular assist device support. Circulation 98:656-662, 1998.

41. Gonzalez-Juanatey JR, Garcia-Acuna JM, Pose A, et al: Reduction of QT and QTc dispersion during longterm treatment of systemic hypertension with enalapril. Am J Cardiol 81:170-174, 1998.

42. Oikarinen L, Nieminen MS, Toivonen L, et al: Relation of QT interval and QT dispersion to regression of echocardiographic and electrocardiographic left ventricular hypertrophy in hypertensive patients: The Losartan Intervention For Endpoint Reduction (LIFE) study. Am Heart J 145:919-925, 2003.

43. Rials SJ, Wu Y, Xu X, et al: Regression of left ventricular hypertrophy with captopril restores normal ventricular action potential duration, dispersion of refractoriness, and vulnerability to inducible ventricular fibrillation. Circulation 96:1330-1336, 1997.

44. Cerbai E, De Paoli P, Sartiani L, et al: Treatment with irbesartan counteracts the functional remodeling of ventricular myocytes from hypertensive rats. J Cardiovasc Pharmacol 41:804-812, 2003.

45. Rials SJ, Wu Y, Ford N, et al: Effect of left ventricular hypertrophy and its regression on ventricular electrophysiology and vulnerability to inducible arrhythmia in the feline heart. Circulation 91:426-430, 1995.

46. Harding JD, Piacentino V 3rd, Gaughan JP, et al: Electrophysiological alterations after mechanical circulatory support in patients with advanced cardiac failure. Circulation 104:1241-1247, 2001.

47. Kreher P, Ristori MT, Corman B, et al: Effects of chronic angiotensin I-converting enzyme inhibition on the relations between ventricular action potential changes and myocardial hypertrophy in aging rats. $J$ Cardiovasc Pharmacol 25:75-80, 1995.

48. Botchway AN, Turner MA, Sheridan DJ, et al: Electrophysiological effects accompanying regression of left ventricular hypertrophy. Cardiovasc Res 60:510-517, 2003.

49. Peschar M, Vernooy K, Vanagt WY, et al: Absence of reverse electrical remodeling during regression of volume overload hypertrophy in canine ventricles. Cardiovasc Res 58:510-517, 2003.

50. Wickenden AD, Kaprielian R, Kassiri Z, et al: The role of action potential prolongation and altered intracellular calcium handling in the pathogenesis of heart failure. Cardiovasc Res 37:312-323, 1998. 
51. Sah R, Ramirez RJ, Backx PH: Modulation of $\mathrm{Ca}^{2+}$ release in cardiac myocytes by changes in repolarization rate: Role of phase-1 action potential repolarization in excitation-contraction coupling. Circ Res 90:165-173, 2002.

52. Kassiri Z, Zobel C, Nguyen T-TT, et al: Reduction of Ito causes hypertrophy in neonatal rat ventricular myocytes. Circ Res 90:578-585, 2002.

53. Sanguinetti MC: Reduced transient outward $\mathrm{K}^{+}$current and cardiac hypertrophy: Causal relationship or epiphenomenon? Circ Res 90:497-499, 2002.

54. Gwathmey JK, Morgan JP: Sarcoplasmic reticulum calcium mobilization in right ventricular pressureoverload hypertrophy in the ferret: Relationships to diastolic dysfunction and a negative treppe. Pflügers Arch 422:599-608, 1993.

55. Maier LS, Brandes R, Pieske B, et al: Effects of left ventricular hypertrophy on force and $\mathrm{Ca}^{2+}$ handling in isolated rat myocardium. Am J Physiol 274:H1361-H1370, 1998.

56. Hu Z, Metaxas D, Axel L: In vivo strain and stress estimation of the heart left and right ventricles from MRI images. Med Image Anal 7:435-444, 2003.

57. Herbots L, Maes F, D'Hooge J, et al: Quantifying myocardial deformation throughout the cardiac cycle: a comparison of ultrasound strain rate, grey-scale M-mode and magnetic resonance imaging. Ultrasound Med Biol 30:591-598, 2004.

58. Arts T, Bovendeerd PH, Prinzen FW, et al: Relation between left ventricular cavity pressure and volume and systolic fiber stress and strain in the wall. Biophys J 59:93-102, 1991. 



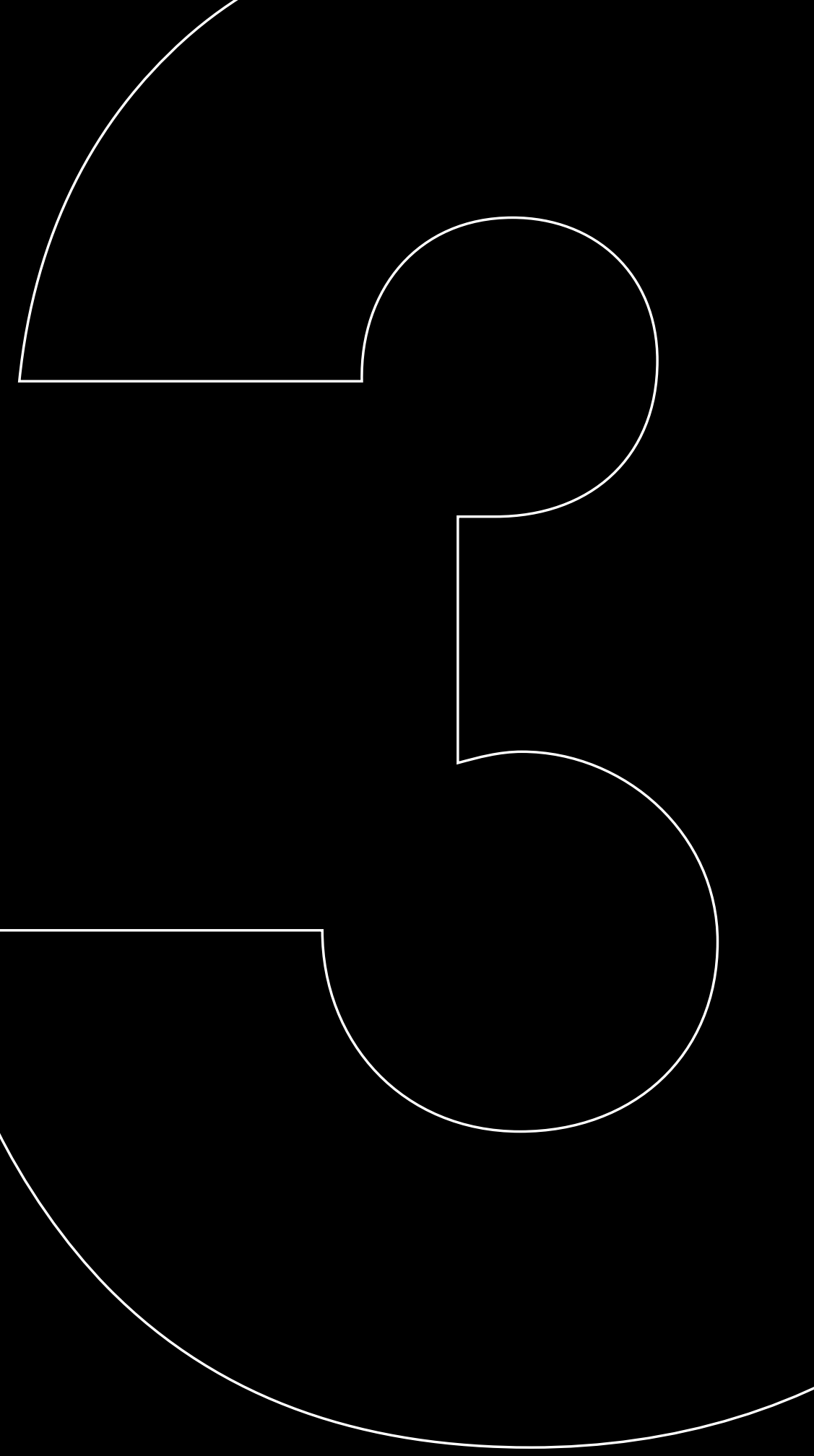


Dirk W. Donker, Paul G.A. Volders, Theo Arts, Bas C.M. Bekkers, Leo Hofstra, Roel L.H.M.G. Spätjens, Jet D. Beekman, Marcel Borgers, Harry J.G.M. Crijns, Marc A. Vos.

Basic Res Cardiol. 2005;100:372-82

Eur Heart J. 2004;25:555-555 Suppl. S. 
End-diastolic myofiber stress and ejection strain increase with ventricular volume overload

Serial in-vivo analyses in dogs with complete atrioventricular block 


\section{ABSTRACT}

Background Myocardial stress and strain are considered primary mechanical stimuli for hypertrophic remodeling. Their values and significance in the intact beating heart during chronic overload remain poorly characterized.

Methods and Results Left-ventricular (LV) dimensions (echocardiography) and pressure (invasive) were simultaneously recorded in anesthetized dogs at sinus rhythm (SR), acute and 1, 2, 6, 12 weeks of atrioventricular block (AVB), leading to structural, electrical and contractile remodeling. Mechanical load of the myocardium was quantified as myofiber stress $\left(\sigma_{f}\right)$, being force along myofiber orientation per cross-sectional area, and natural myofiber strain $\left(\mathrm{e}_{\mathrm{f}}\right)$, being change in natural logarithm of myofiber length (I) divided by its reference length: $e_{f}=\ln \left(I / I_{\text {ref }}\right)$. Time courses of $\sigma_{f}$ and $e_{f}$ were calculated from LV pressure and dimensions, using a validated mathematical model of cardiac mechanics. End-diastolic $\sigma_{f}$ increased from $2.0 \pm 0.1 \mathrm{kPa}$ at $\mathrm{SR}$ to $3.4 \pm 0.3 \mathrm{kPa}$ at acute $A V B$, remaining elevated for $>6$ weeks. Systolic $\sigma_{f}$ was not affected by AVB. Ejection strain rose instantly upon $A V B$, reaching a maximum at 2 weeks: $0.24 \pm 0.02$ vs. $0.10 \pm 0.01$ at $S R$. The increase of myofiber stroke work $\left(\sigma_{f}-e_{f}\right.$ loop area) from $3.1 \pm 0.3$ at $S R$ to $6.0 \pm 0.5 \mathrm{~kJ} / \mathrm{m}^{3} /$ beat at 1 week AVB was attributed mainly to an increase of strain during ejection. Stroke work and ejection strain remained elevated up to 12 weeks. The rate of LV-mass increase was maximal $(2.2 \pm 0.4 \mathrm{~g} /$ day $)$ at 1 week AVB.

Conclusions Serial mechanical phenotyping is feasible in the intact anesthetized dog with chronic ventricular overload. Our new approach yields values of mechanical load that are comparable to those found in isolated myocardium by others. In chronic AVB, both end-diastolic myofiber stress and ejection strain are increased. Early increases of both parameters coincide with peak hypertrophic growth, suggesting their important role for mechanotransduction. Peak systolic $\sigma_{f}$ is likely not important for hypertrophy in this model, since it does not change throughout the experiment. 


\section{INTRODUCTION}

It has long been recognized that cardiac hypertrophy is the adaptive response to altered hemodynamic loading and that it is load-specific (e.g., pressure versus volume overload) $[9,17]$. In most, if not all, experimental models of overload, stress and strain are primary mechanical stimuli for hypertrophic remodeling $[11,21,24]$. Myofiber stress is defined as mechanical force along the myofiber direction per cross-sectional area and natural myofiber strain as the change in the logarithm of myofiber length divided by its reference length. In cultured neonatal myocytes, stress and strain trigger mitogen-activated protein- and other kinase cascades, protooncogene expression and increased protein turnover $[15,27,38]$. Based on these elementary studies, the nature, degree, direction and timing of mechanical load during the cardiac cycle all appear crucial input factors for the activation of biochemical signals $[15,21,27,38]$.

Despite the plethora of in vitro data on mechanotransduction over the last 15 years, exact quantification of mechanical stimuli at the tissue level and their significance for remodeling in the intact beating heart remain poorly identified, mainly due to methodological constraints. Calculation of wall stress finds widespread use, but is often based on isotropic tissue behavior or is limited to the analysis of meridional or circumferential components of stress. Thereby, the assessment of myocardial mechanics strongly depends on ventricular geometry and the site of measurement. Invasive analysis of stress and strain with intramyocardially applied probes is cumbersome and limited to acute experiments or ex vivo studies only, which makes them unsuitable for serial analyses in the same patient or experimental animal.

In recent years, we and others have extensively studied a canine model of volume overload due to complete atrioventricular block (AVB). Important electrical, contractile and structural alterations of the myocardium are found in this model $[28,34,35,37]$. Myocardial adaptation is associated with an enhanced susceptibility to torsades-de-pointes ventricular arrhythmias $[25,34,35]$ and sudden cardiac death [32]. Most dogs exhibit compensated contractile function in the chronic stage of AVB [37]. The impetus to the present study was the lack of quantitative information on the mechanical stimuli, imposed on the myocardium in $A V B$, which potentially trigger structural and functional adaptations. Therefore, we sought to circumvent the constraints of intramyocardial measurements in vivo by factoring ventricular dimensions and pressure into a validated mathematical model of myocardial mechanics [1]. By doing so, we were able to estimate stress and strain in the myofibers of the tissue in a serial manner before and after induction of AVB. Thus, cardiac mechanical phenotyping was made possible in the intact anesthetized dog with chronic ventricular overload. 


\section{METHODS}

Experiments were conducted in accordance with the Dutch Law on Animal Experimentation and the European Directive for the Protection of Vertebrate Animals Used for Experimental and Other Scientific Purposes (86/609/EU). The Committee for Experiments on Animals of Maastricht University approved the experiments.

\section{Experimental model}

Adult mongrel dogs of either gender $(27 \pm 1 \mathrm{~kg})$ were used. After overnight fasting, premedication ( $0.15 \mathrm{mg} / \mathrm{kg}$ acepromazine, $0.4 \mathrm{mg} / \mathrm{kg}$ methadon, $0.06 \mathrm{mg} / \mathrm{kg}$ atropine i.m.) was administered. Animals were anesthetized with pentobarbital $(20 \mathrm{mg} / \mathrm{kg}$ i.v.) and mechanically ventilated with a mixture of oxygen (40\%), nitrous oxide $(60 \%)$ and halothane $(0.5-1 \%$ vapor concentration). A complete description of the perioperative care has been given before [35]. In 8 dogs AVB was induced by radio-frequency catheter ablation of the His bundle. All animals were studied serially at sinus rhythm (SR; control), acute AVB and 1, 2, 6 and 12 weeks thereafter. A standard 6-lead ECG was registered to synchronize left-ventricular (LV) echocardiographic and pressure recordings. At the end of the experiments the animals were sacrificed, their hearts excised and weighed. LV myocytes were enzymatically isolated to determine cell length and width in 50 randomly selected cells per animal. In addition, 7 dogs were sacrificed at SR for control purposes to compare cellular dimensions and heart weights at autopsy.

\section{LV pressure}

A 7F catheter (Sentron, Roden, Netherlands) was introduced into the LV cavity via the femoral artery to monitor pressure $\left(\mathrm{p}_{\mathrm{LV}}\right)$. End-diastolic $\left(\mathrm{p}_{\mathrm{LV}, \mathrm{ed}}\right)$, peak-systolic pressure $\left(\mathrm{p}_{\mathrm{LV}, \mathrm{sys}}\right)$, and the maximum rate of pressure rise $\left(\mathrm{dp}_{\mathrm{LV}} / \mathrm{dt}_{\text {max }}\right)$ were determined off-line using custom-made software (IDEEO, Maastricht University).

\section{LV dimensions}

Transthoracic echocardiography was performed (3.5 MHz transducer, Megas Ultrasound System, Esaote, Genoa, Italy). LV long axis inner diameter was measured from left parasternal long-axis views as the minimum (systole) and maximum (diastole) distance between the inner wall of the apex and the mitral-valve plane. LV M-mode recordings were derived from right parasternal short-axis views in the mid-papillary plane. Video frames were digitized and processed using custom-made software (IDEEQ, Maastricht University), allowing for the determination of endo- and epicardial borders, and short-axis diameters and wall thickness. Mean wall thickness was the average of 
posterior and septal wall thickness. LV mass was estimated according to Reichek et al. [23]. The estimation of apical wall thickness and the calculation of LV cavity $\left(\mathrm{V}_{\mathrm{LV}}\right)$ and wall volume $\left(\mathrm{V}_{\mathrm{w}}\right)$ are described in the Appendix.

\section{Myofiber mechanics}

Conventionally, mechanical load of the cardiac wall is quantified by hoop stress, i.e.,the stress in circumferential direction, using models of wall mechanics $[6,16]$, based on Laplace's law. Hoop stress provides an underestimate of myofiber stress by about 40\% [1]. Myofiber stress is defined as the force of the myofibers in the wall, as normalized per cross-sectional area. For most clinical applications that underestimation is not a problem, because the related correction factor is about fixed for a given heart. Changes in hoop stress follow changes in myofiber stress. In the present study, myofiber stress is quantified because this stress compares directly to the stress measured in experiments on isolated cardiac muscle [3], thus indicating its physiological importance. Myofiber stress is considered to be related to physiological processes in the neighborhood of the cells in the tissue, such as adaptation and remodeling. Complicated finite element models may provide the best estimates of myofiber stress [14,30], but they are difficult to handle. As a compromise between accuracy and simplicity a model [1] has been used to calculate average myofiber stress $\left(\sigma_{f}\right)$ from LV pressure $\left(\mathrm{p}_{\mathrm{LV}}\right)$ and the ratio of cavity volume $\left(\mathrm{V}_{\mathrm{LV}}\right)$ to wall volume $\left(\mathrm{V}_{\mathrm{w}}\right)$ as illustrated in Figure 1:

Myofiber stress:

$$
\sigma_{f}=p_{L V}\left(\frac{1+3 V_{L V}}{V_{W}}\right)
$$

In a theoretical evaluation [1], the accuracy of the myofiber stress thus calculated was approximately $5 \%$, showing that the major geometric determinant was the ratio $V_{L V} / V_{w^{\prime}}$, whereas all other geometric properties appeared of secondary importance. In the present study, the term "myofiber" designates a tissue bundle of interconnected cardiomyocytes embedded in an extracellular matrix. During systole, cardiomyocytes account for the active stress development, and during diastole, the passive stress development is partitioned between the extracellular matrix (collagen) and the cytoskeletal components contributing to the intrinsic passive properties of the cardiomyocyte (titin) [8]. The load bearing myofibers that contribute to cavity pressure are assumed to be mainly parallel to the curved wall surface. Average natural strain of the myofibers $\left(e_{f}\right)$ has been defined as the natural logarithm of myofiber length $(I)$, relative to its reference length $\left(I_{\text {ref }}\right)$. Using the physical principle of conservation of energy, it is derived from Eq. 1 that [1]: 

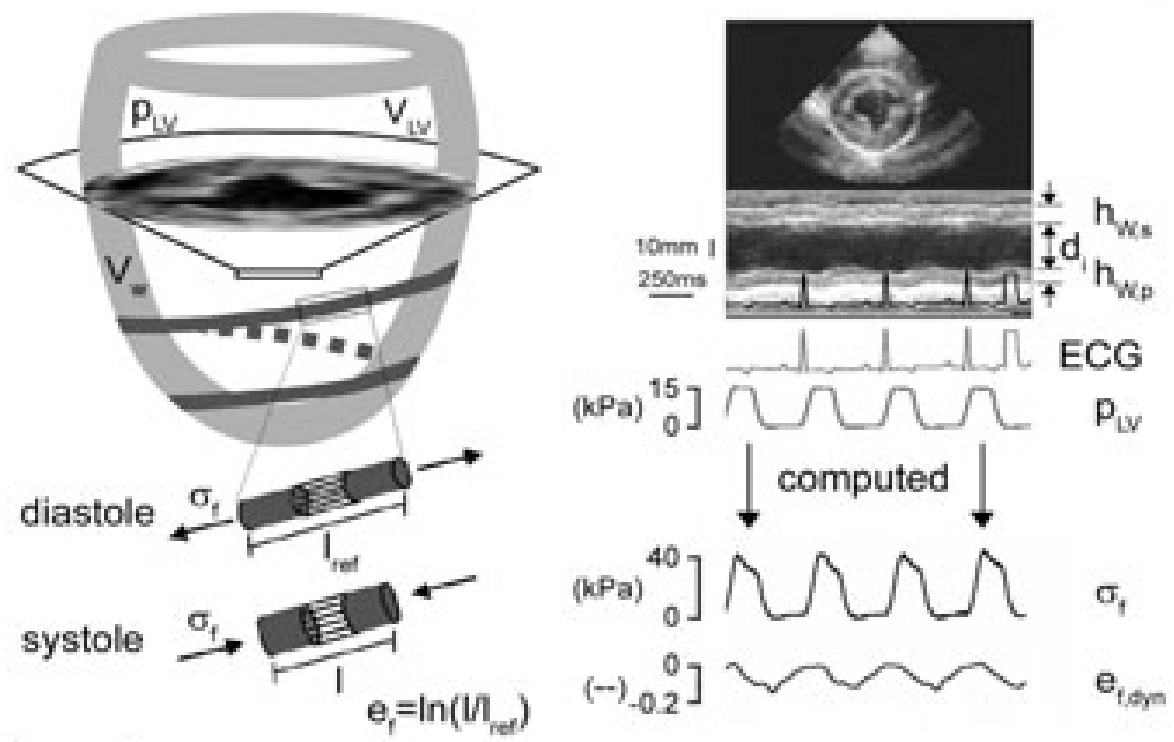

Figure 1 | Model of myofiber mechanics. Left side, myofibers (dark gray) are embedded in a truncated ellipsoid representing the LV. Dynamic changes of myofiber strain $\left(e_{f}\right)$ defined as the ratio of instantaneous myofiber length (I) (e.g., systole) compared to diastolic length $\left(\left.\right|_{\text {ref }}\right)$ are largely determined by myofiber stress $\left(\sigma_{f}\right)$. Echocardiographic LV dimensions are assessed in the mid-papillary plane (short-axis scan). Right side, from top to bottom, echocardiographic M-mode serves to determine LV septal $\left(h_{w . s}\right)$, posterior wall thickness $\left(h_{w, p}\right)$ and inner diameter $\left(\mathrm{d}_{\mathrm{i}}\right)$ in order to calculate LV-cavity $\left(\mathrm{V}_{\mathrm{LV}}\right)$ and wall volume $\left(\mathrm{V}_{\mathrm{w}}\right)$, incorporating long axes (not depicted). $\mathrm{LV}$ dimensions are ECG synchronized with LV-cavity pressure $\left(\mathrm{p}_{\mathrm{LV}}\right)$. Mathematical modeling of these global LV quantities provides values of $\sigma_{f}$ and $e_{f}$ throughout the cardiac cycle.

\section{Myofiber strain:}

$$
e_{f}=\operatorname{In}\left(\frac{I}{I_{r e f}}\right)=\frac{1}{3} \operatorname{In}\left(\frac{1 / 3 V_{W}+V_{L V}}{1 / 3 V_{W, \text { ref }}+V_{L V, \text { ref }}}\right)
$$

This equation for myofiber strain has been validated in the open-chest canine heart [4], where fiber strain was measured directly on the epicardial surface by video.

For dynamic strain changes during the cardiac cycle $\left(e_{\mathrm{f}, \mathrm{dyn}}\right)$, we used end-diastole as the reference state. Ejection strain $\left(\Delta \mathrm{e}_{\mathrm{f}, \mathrm{e}}\right)$, representing myofiber shortening during systole, was defined as the decrease of $e_{f, d y n}$ during ejection: 


$$
e_{f, d y n}=\operatorname{In}\left(\frac{I}{I_{e d}}\right)=1 / 3 \operatorname{In}\left(\frac{1 / 3 V_{W}+V_{L V}}{1 / 3 V_{W}+V_{L V, e d}}\right)
$$

Ejection strain:

$$
\Delta e_{f, e j}=\operatorname{In}\left(\frac{I_{e d}}{I_{e s}}\right)=1 / 3 \operatorname{In}\left(\frac{1 / 3 V_{W}+V_{L V, e d}}{1 / 3 V_{W}+V_{L V, e s}}\right)
$$

The subscripts 'ed' and 'es' refer to end diastole and end systole, respectively. To compare the enddiastolic states of cardiac cycles between different experimental time points, myofiber strain of the end-diastolic midwall circumference is compared to its length at $\mathrm{SR}\left(\mathrm{e}_{\mathrm{f}, \mathrm{ed}}\right)$. It is used:

End-diastolic strain:

$$
e_{f, e d}=\operatorname{In}\left(\frac{I_{e d}}{I_{e d, S R}}\right)=1 / 3 \operatorname{In}\left(\frac{1 / 3 V_{W}+V_{L V, e d}}{1 / 3 V_{W, S R}+V_{L V, e d, S R}}\right)
$$

During the cardiac cycle (Eq. 3), wall volume remains constant. Note that, as compared to LV-cavity volume $\left(\mathrm{V}_{\mathrm{LV}, \text { ed,SR }}\right)$ and LV-wall volume $\left(\mathrm{V}_{\mathrm{W}, \mathrm{SR}}\right)$ at end-diastole during SR (Eq. 4), both LV-cavity volume at end-diastole $\left(\mathrm{V}_{\mathrm{LV}, \mathrm{ed}}\right)$ and $\mathrm{V}_{\mathrm{W}}$ may change due to structural remodeling after $\mathrm{AVB}$.

During diastole, the short-axis cross-section of the LV deforms to non-circularity due to gravity. Thus, M-mode-derived diastolic cavity diameter is not appropriate for determination of cavity cross-sectional area. During isovolumic contraction, cavity volume, cavity area and cavity circumference all remain practically constant, whereas the cross-section becomes practically circular. Thus, M-mode-determined diameter during the isovolumic contraction phase is used to estimate end-diastolic volume. In this analysis, the latter diameter is determined at the moment $p_{\mathrm{Lv}}$ reaches half of its systolic peak value.

Total LV stroke work, represented by the area of the $\mathrm{p}_{\mathrm{LV}}-\mathrm{V}_{\mathrm{LV}}$ loop, equals wall volume times myofiber stroke work. Myofiber stroke work $w_{f}$ is computed as:

Myofiber stroke work:

$$
w_{f}=\int_{\text {cycle }} \sigma_{f} d e_{f}
$$




\section{Statistical analysis}

Data are presented as mean \pm SEM. Differences by repeated measures ANOVA or unpaired Student's t-test (post-mortem heart weights and micromorphometry) are considered significant when $\mathrm{P}<0.05$.

\section{RESULTS}

\section{Hemodynamics}

Induction of AVB reduced the heart rate from $111 \pm 5$ at SR to $56 \pm 3$ beats/min at idioventricular rhythm $(P<0.05$; Table 1). Acute bradycardia decreased cardiac output from $2.8 \pm 0.4$ to $1.6 \pm 0.2$ $L / m i n(P<0.05)$ and was accompanied by an elevation of $p_{L V, e d}(0.7 \pm 0.0$ to $1.1 \pm 0.1 \mathrm{kPa} ; P<0.05)$ [35]. Systolic $p_{L V}$ remained unaltered (Figure 2). Total LV stroke work $\left(\mathrm{p}_{\mathrm{LV}}-\mathrm{V}_{\mathrm{LV}}\right.$-loop area in Figure 2) showed an increase that became significant from acute AVB onwards (from $0.30 \pm 0.04 \mathrm{~J} /$ beat at $S R$ to $0.56 \pm 0.06 \mathrm{~J} /$ beat at 1 week; $\mathrm{P}<0.05$ ).

LV contractile performance was enhanced during AVB, as evident from the instantaneous increase of fractional shortening and the more delayed, but significant rise of $\mathrm{dp}_{\mathrm{Lv}} / \mathrm{dt}_{\max }$ (Table 1). Both

Table 1 Hemodynamic and electrocardiographic measurements

\begin{tabular}{|c|c|c|c|c|c|c|}
\hline & SR & AAVB & 1 week & 2 weeks & 6 weeks & 12 weeks \\
\hline \multicolumn{7}{|l|}{ LV pressure } \\
\hline Peaksystole, $\mathrm{kPa}$ & $12.8 \pm 0.4$ & $12.8 \pm 0.7$ & $13.0 \pm 0.4$ & $13.4 \pm 0.3$ & $14.0 \pm 0.2$ & $11.9 \pm 0.2$ \\
\hline Enddiastole, $\mathrm{kPa}$ & $0.7 \pm 0.0$ & $1.1 \pm 0.1^{\star}$ & $1.1 \pm 0.1^{*}$ & $1.0 \pm 0.1^{*}$ & $1.1 \pm 0.1^{\star}$ & $0.8 \pm 0.1$ \\
\hline Maximum rise, $\mathrm{kPa} / \mathrm{s}$ & $243 \pm 28$ & $239 \pm 30$ & $371 \pm 37^{\star}$ & $364 \pm 26^{\star}$ & $321 \pm 19^{\star}$ & $230 \pm 6$ \\
\hline Cardiac output, L/min & $2.8 \pm 0.4$ & $1.6 \pm 0.2^{\star}$ & $1.5 \pm 0.3^{*}$ & $2.2 \pm 0.2$ & $2.7 \pm 0.2$ & $2.5 \pm 0.4$ \\
\hline Fractional shortening, $\%$ & $20.4 \pm 1.8$ & $26.9 \pm 2.1^{*}$ & $37.2 \pm 3.9^{\star}$ & $37.7 \pm 4.3^{\star}$ & $35.7 \pm 3.6^{\star}$ & $32.0 \pm 3.3^{\star}$ \\
\hline Total LV stroke work, J/beat & $0.30 \pm 0.04$ & $0.43 \pm 0.09^{\star}$ & $0.56 \pm 0.06^{\star}$ & $0.61 \pm 0.05^{\star}$ & $0.78 \pm 0.07^{\star}$ & $0.72 \pm 0.13^{\star}$ \\
\hline Heart rate, beats/min & $111 \pm 5$ & $56 \pm 3^{\star}$ & $42 \pm 4^{\star}$ & $42 \pm 3^{\star}$ & $43 \pm 2^{\star}$ & $39 \pm 2^{\star}$ \\
\hline PP interval, ms & $549 \pm 26$ & $416 \pm 30^{*}$ & $577 \pm 23$ & $600 \pm 29$ & $620 \pm 46$ & $586 \pm 29$ \\
\hline
\end{tabular}

SR sinus rhythm; AAVB acute complete atrioventricular block; LV left ventricle; ${ }^{*} \mathrm{P}<0.05$ vs. SR 


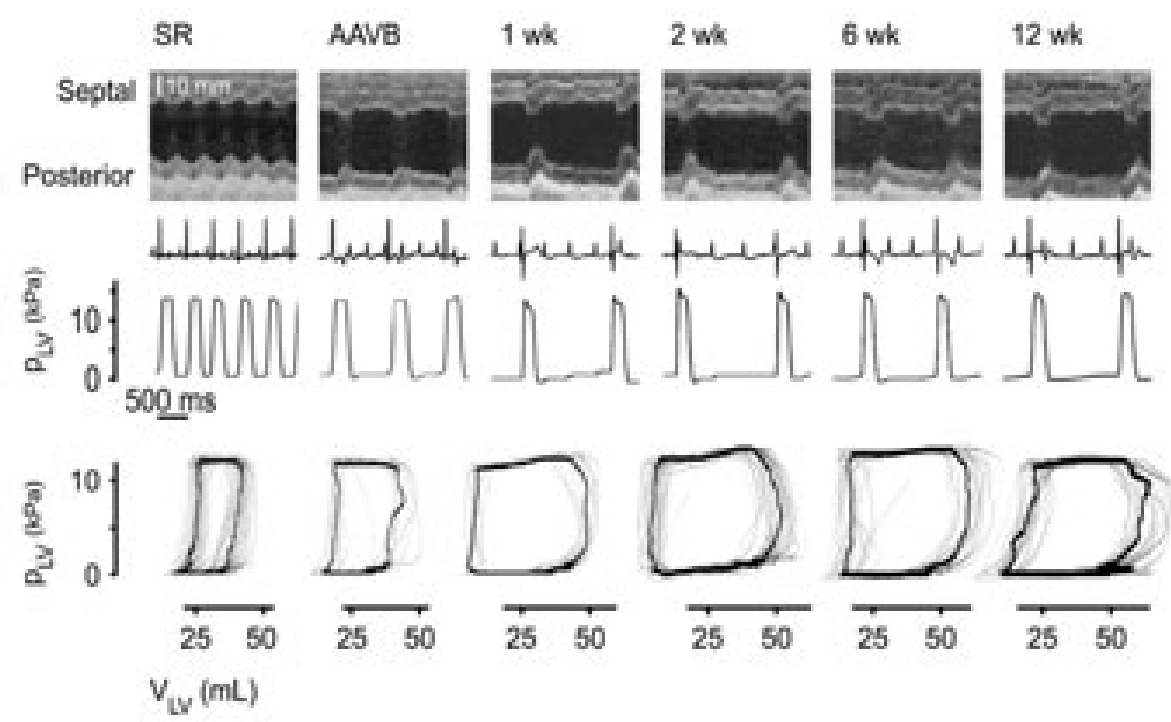

Figure 2 | Upper panels, M-mode echocardiograms of the LV septal and posterior wall synchronized with $p_{L V}$ in a representative anesthetized dog. Upon the creation of AVB, a narrow QRS-complex idioventricular rhythm ensued. Bradycardia was most pronounced at $\geq 1$ week AVB. Note that the PP interval was shortened in the acute phase (AAVB). Lower panels, $p_{L V}-V_{L V}$ loops at the same time points. The area bounded by the $p_{L V}-V_{L V}-$ loop trajectory represents total LV stroke work.

parameters reached their maximal values at 1-2 weeks AVB. This contractile enhancement could be due to an elevated preload and neurohumoral activation [35]. The latter is reflected in the decreased PP interval during acute AVB ( $416 \pm 30$ vs. $549 \pm 26$ ms at $S R ; P<0.05$; Table 1 ; Figure 2). The early decline of cardiac output, expressing incomplete circulatory compensation, was largely overcome by 2 weeks AVB. Hemodynamic status stabilized in the chronic phase thereafter despite a further slowing of heart rate (Table 1; Figure 2).

\section{LV dimensions and hypertrophy}

Serial echocardiography revealed that diastolic LV-cavity dimensions and wall thickness remained unchanged in the acute phase after induction of AVB (Figure 2). During chronic AVB, diastolic dimensions increased mainly along the longitudinal axis, whereas wall thickness did not significantly alter (Table 2). Echocardiographic LV mass had already increased after 1 week. LV-mass increment per day was most prominent in the first week after AVB. Thereafter, it still increased, but far more gradual (Figure 3). 
Table 2 | Left-ventricular dimensions

\begin{tabular}{|c|c|c|c|c|c|c|}
\hline & SR & AAVB & 1 week & 2 weeks & 6 weeks & 12 weeks \\
\hline \multicolumn{7}{|c|}{ Short axis inner diameter } \\
\hline Diastole, $\mathrm{mm}$ & $39.8 \pm 1.4$ & $40.9 \pm 1.6$ & $41.6 \pm 1.5$ & $42.4 \pm 1.4$ & $44.4 \pm 1.7$ & $45.9 \pm 2.0$ * \\
\hline Systole, mm & $31.7 \pm 1.3$ & $29.8 \pm 0.9$ & $26.1 \pm 1.9$ * & $26.5 \pm 1.8^{*}$ & $28.5 \pm 1.8^{*}$ & $31.1 \pm 1.7$ \\
\hline \multicolumn{7}{|c|}{ Long axis inner diameter } \\
\hline Diastole, mm & $70.3 \pm 2.5$ & $68.4 \pm 2.7$ & $75.2 \pm 1.9^{*}$ & $75.7 \pm 1.6^{*}$ & $86.7 \pm 1.8^{*}$ & $90.3 \pm 1.4$ * \\
\hline Systole, mm & $63.9 \pm 2.4$ & $55.7 \pm 2.1^{*}$ & $54.8 \pm 4.4^{*}$ & $52.5 \pm 3.7^{*}$ & $64.2 \pm 2.1$ & $69.3 \pm 2.9$ * \\
\hline \multicolumn{7}{|c|}{ Mean wall thickness } \\
\hline Diastole, $\mathrm{mm}$ & $9.6 \pm 0.5$ & $9.3 \pm 0.7$ & $9.5 \pm 0.4$ & $9.4 \pm 0.4$ & $9.3 \pm 0.3$ & $9.2 \pm 0.4$ \\
\hline Systole, mm & $13.1 \pm 0.8$ & $13.9 \pm 1.0$ & $15.2 \pm 1.2$ & $14.9 \pm 1.1$ & $14.3 \pm 1.1$ & $13.8 \pm 0.6$ \\
\hline
\end{tabular}

SR indicates sinus rhythm; AAVB acute complete atrioventricular block. ${ }^{*} \mathrm{P}<0.05$ vs. SR

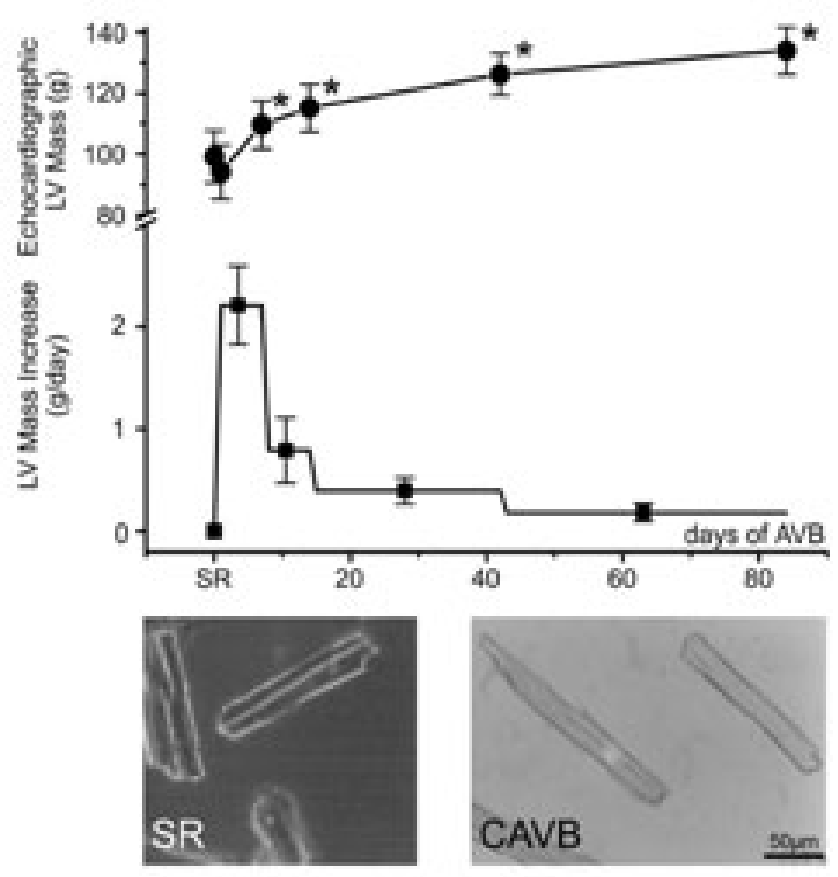

Figure $3 \mid$ Time course of hypertrophy after AVB. Note that the LV-mass increase per day (lower trace) was most pronounced in the first week. Photomicrographs illustrate predominant lengthening of LV cardiomyocytes during chronic AVB. Note that the scale bar applies to both photomicrographs. ${ }^{*} P<0.05$ vs. SR 

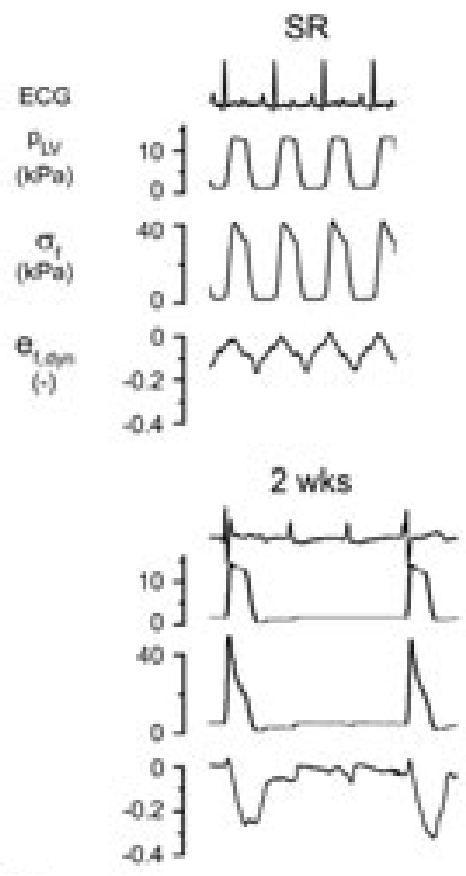
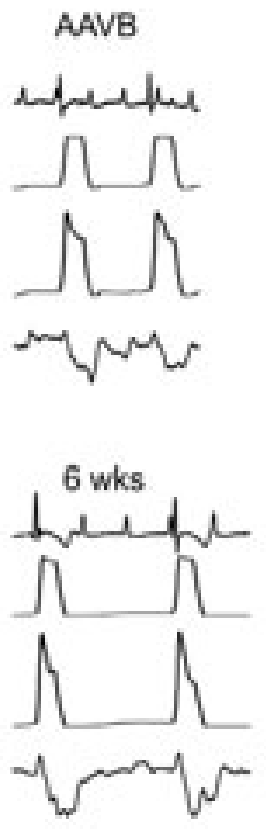

1 wk
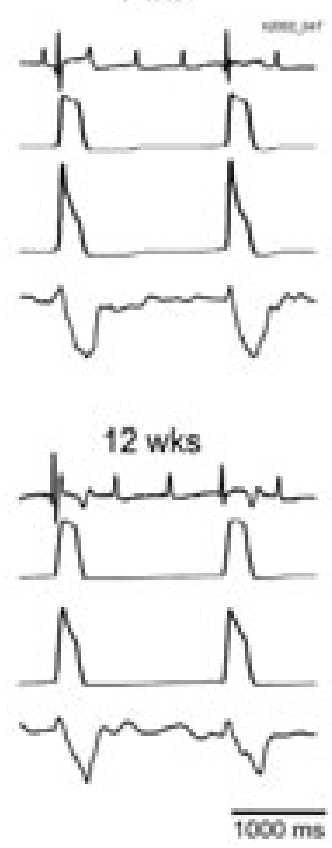

Figure $4 \mid$ Representative examples of myofiber mechanics throughout the cardiac cycle in an individual dog before and after AVB. $\sigma_{f}$ and $e_{f}$ are time-linked to $E C G$ and $p_{L v}$.

At sacrifice after 12 weeks AVB, total heart weights $(323 \pm 13 \mathrm{~g})$ and heart-weight-to-body-weight ratios $(12.0 \pm 0.3 \mathrm{~g} / \mathrm{kg})$ were significantly larger than those of the control population of dogs with normal SR $(216 \pm 8 \mathrm{~g}$ and $8.1 \pm 0.3 \mathrm{~g} / \mathrm{kg}$, respectively; both $\mathrm{P}<0.05)$.

Micromorphometry showed predominant cardiomyocyte lengthening: $161 \pm 4 \mu \mathrm{m}$ at 12 weeks AVB vs. $139 \pm 5 \mu \mathrm{m}$ at $S R ;+16 \% ; P<0.05)$. Cellular width changed to a lesser degree $(+11 \% ; 25.8 \pm 0.7$ vs. $23.3 \pm 0.3 \mu \mathrm{m}$, respectively; $\mathrm{P}<0.05$; Figure 3).

\section{Myofiber mechanics}

Stable ECG-synchronized tracings of $\sigma_{f}$ and $e_{f}$ throughout the cardiac cycle were reproducibly obtained in all animals at control, acute and chronic AVB. Examples for a representative dog are shown in Figure 4.

Peak-systolic $\sigma_{f}$ was not significantly affected by the presence of AVB. In contrast, end-diastolic $\sigma_{f}$ increased instantaneously from $2.0 \pm 0.1 \mathrm{kPa}$ at $\mathrm{SR}$ to $3.4 \pm 0.3 \mathrm{kPa}$ at acute $A V B$, remained as high 

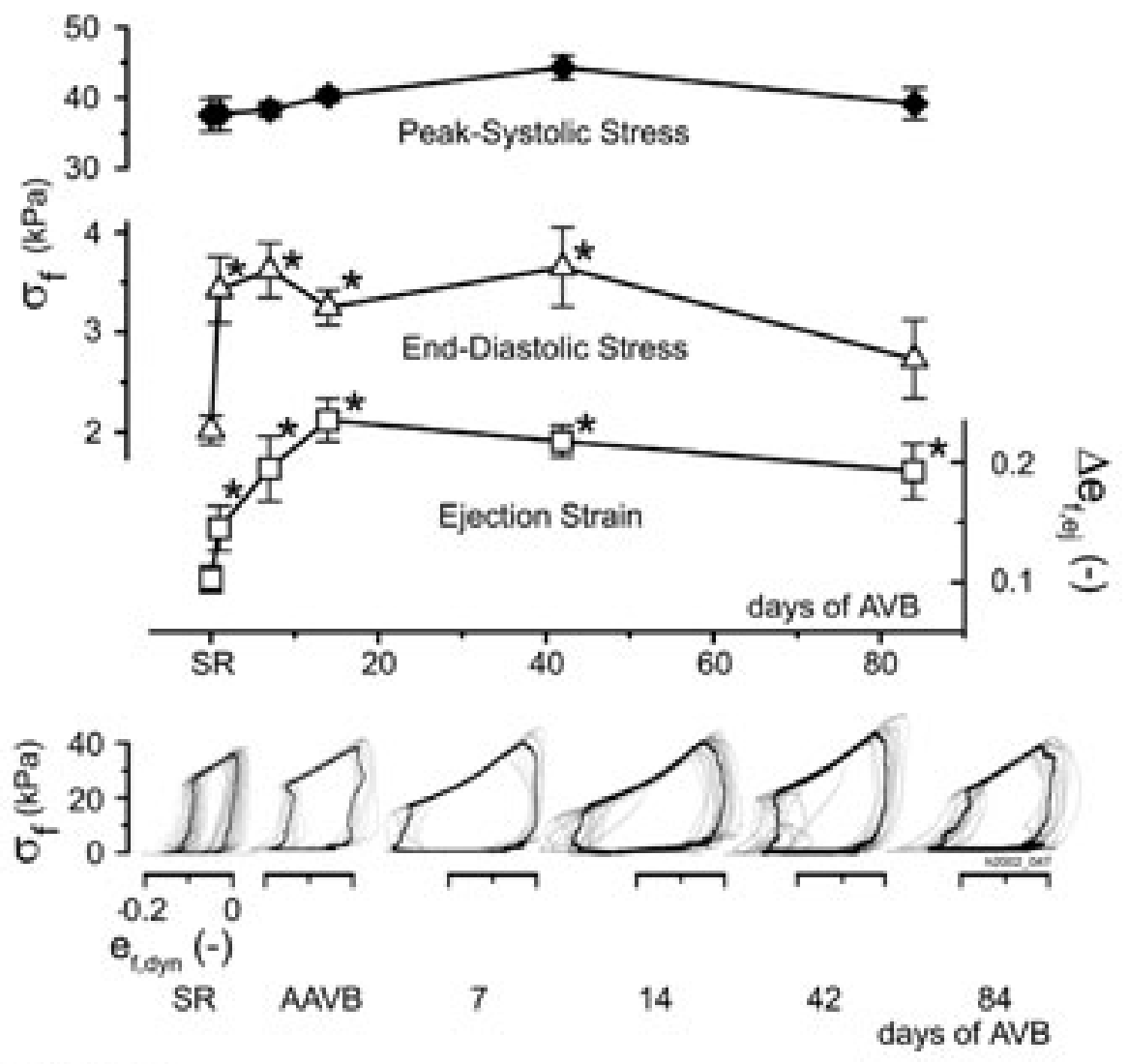

Figure $5 \mid$ Time course of myofiber mechanics before and after AVB. Upper panel, average values $\left(n_{\text {dogs }}=8\right)$ for $\sigma_{f}$ at peak systole and end diastole, and ejection strain. Lower panel, representative time-dependent changes of $\sigma_{f}-e_{f}$ loops in an individual dog. The area bounded by the $\sigma_{f}-e_{f}$ loop trajectory represents myofiber stroke work per beat, standardized per unit myocardial volume. ${ }^{*}<<0.05$ vs. SR.

until 6 weeks, with little change thereafter $(3.7 \pm 0.4$ to $2.9 \pm 0.4 \mathrm{kPa}$; 6 to 12 weeks, respectively; $\mathrm{P}=n \mathrm{~s} ;$ Figure 5). Upon $A V B, \Delta \mathrm{e}_{\mathrm{f}, \mathrm{j}}$ rose rapidly, reaching a maximum at 2 weeks: $0.24 \pm 0.02 \mathrm{vs}$. $0.10 \pm 0.01$ at $S R(P<0.05 ;$ Figure 5$)$, and was persistently high throughout the chronic phase.

Myofiber stroke work, represented by the area bounded by the $\sigma_{f}-e_{f}$ loop, also increased rapidly after AVB: $4.3 \pm 0.7$ vs. $3.1 \pm 0.3 \mathrm{~kJ} / \mathrm{m}^{3} /$ beat at $S R(P<0.05)$. This parameter attained its maximum at 1 week AVB $\left(6.0 \pm 0.5 \mathrm{~kJ} / \mathrm{m}^{3} /\right.$ beat) and remained elevated up to 12 weeks after AVB (Figure 5). 
We also examined end-diastolic strain, which represents the resultant of end-diastolic stretch plus hypertrophic myofiber growth over time. In the acute phase of AVB, so when only stretch (and no hypertrophy) was present, end-diastolic strain measured $0.01 \pm 0.01$. At 1 week, it had already increased to $0.04 \pm 0.02$. Thereafter, it increased steadily to $0.15 \pm 0.03$ at 12 weeks AVB in major part by the influence of hypertrophy.

\section{DISCUSSION}

In the present study we serially quantified the mechanical stimuli stress and strain in intact anesthetized dogs with chronic volume overload and hypertrophy. Synchronized measurements of LV dimensions and pressure were factored into a mathematical model of myocardial mechanics [1]. This approach allows serial intra-individual quantification of tissue mechanics in vivo. The values we obtained are comparable to values derived from preparations of isolated myocardium [3].

After the induction of $A V B$, increases of end-diastolic stress, end-diastolic strain and ejection strain were found to be the main mechanical changes, whereas peak systolic stress remained unaltered. Early increases of the former quantities coincided with peak hypertrophic growth in the first week after AVB.

\section{Mechanical changes and ventricular remodeling in dogs with AVB}

The acute decrease of heart rate imposed by AVB elevated end-diastolic pressure by the prolonged diastolic filling phase. As an immediate consequence, end-diastolic $\sigma_{f}$ almost doubled. Also directly after $A V B$, a subtle, but significant increase of the end-diastolic strain was observed by 0.01 at acute $A V B$ and by 0.04 at 1 week, respectively. At this latter time-point, the contribution of stretch could not be quantitatively distinguished from myofiber deformation by growth, which is inherent to the serial assessment of diastolic strain during hypertrophic growth. However, these data suggest that end-diastolic stretch of the LV myocardium after AVB can increase to somewhere between 0.01 and 0.04 above control levels.

Stretch applied to cardiomyocyte preparations, ranging between 0.005 and 0.10 , has been reported to induce significant hypertrophic responses $[27,38]$. Others have described the transmural distribution of end-diastolic growth strains ranging between 0.10 (circumferential) and 0.15 (longitudinal) in dogs after 3.6 weeks of aortocaval fistula, which is similar to our data on end-diastolic strain at 6 weeks AVB (0.12) [22]. In that study [22], the composite nature (i.e., stretch and growth) of diastolic strain during developing hypertrophy has been circumvented by matching preload either by volume suppletion or bleeding, thereby setting the stretch component to a fixed value. In our study, stretch likely follows a similar temporal pattern as end-diastolic $\sigma_{f}$ in the chronic phase of AVB. This assumption is supported by the finding that diastolic stiffness, calculated from the slope 
of the $\sigma_{\mathrm{f}}-\mathrm{e}_{\mathrm{f}}$ loop at end diastole, remained unaltered after AVB $(16 \pm 2 \mathrm{kPa}$ at 12 weeks AVB vs. $24 \pm 7 \mathrm{kPa}$ at $S R ; P=n s)$. This is in line with previous work in dogs with AVB [20] and suggests that stress can directly be transduced into strain, not hampered by increased myocardial stiffness. In dogs with aortocaval fistula, this concept does not hold, since increased myocardial stiffness and diastolic dysfunction influence diastolic stress-strain relations [2].

The increased preload during AVB did not only alter diastolic mechanics, but also influenced the systolic phase. In acute $A V B$, increased preload enhanced contractility by the Frank-Starling mechanism. Contractility was further stimulated by elevated serum levels of norepinephrine [35]. Independent from preload and neurohumoral stimulation, increased $\mathrm{Ca}^{2+}$ release from the sarcoplasmic reticulum adds to the contractile performance in chronic $A V B$, especially during bradycardia [28]. As a mechanical corollary of enhanced contractility, ejection strain rose rapidly after AVB. At 1-2 weeks, it had more than doubled to approximately $27 \%$ myofiber shortening during ejection (compared to $11 \%$ at SR), despite an unchanged afterload. At 12 weeks, ejection strain was still elevated (approximately 21\%) and likely influenced by the apposition of new sarcomeres. For comparison, in dogs with compensated hypertrophy due to aortocaval fistula, systolic strains only tend to increase above normal values [22]. Although volume overload has traditionally been associated with alterations of diastolic mechanics [9], it has been recognized that the myocardium may be 'sensing' the stimulus to hypertrophic growth throughout the cardiac cycle, including systole $[11,21,36]$.

Further experiments are needed to compare the time course of myofiber mechanics to changes of load-dependent biomarkers. On theoretical grounds, ejection strain (which incorporates both short- and long-axis dimensions) has advantages over commonly used one-dimensional parameters like fractional shortening. It is a better representative of myocardial load during systole and relates more closely to load-dependent myocardial alterations.

Based on the temporal relationship between increasing myofiber mechanics and LV mass, enddiastolic $\sigma_{f}$ and strain, and ejection strain are candidate mechanical stimuli for ventricular hypertrophy after AVB, as suggested for other models of volume overload $[7,11,22]$. To date a cellular sensor of $\sigma_{f}$ is not known to exist, and it could well be that any cellular transducer is in fact an $e_{f}$ transducer.

It is clear that the primary stimuli for hypertrophy may also be non-mechanical. Whereas neurohumoral stimuli and cardiac growth factors are obvious candidates, it remains to be determined whether they act independently from mechanical load in the beating heart. Studies in humans [26] and pigs [18] have confirmed the intricate relationships between ventricular mechanical stress, growth-factor formation and hypertrophy, showing that the contributive roles of angiotensin II, endothelin-1 and insulin-like growth factor 1 are selectively linked to both the type of hemodynamic overload and to ventricular function. Future studies should elucidate the differential role of diastolic and systolic $\sigma_{f}$ and $e_{f}$ for hypertrophy programs during chronic cardiac overload in vivo. 
Based on the present finding of a persistent doubling of myofiber stroke work in AVB, which is related to local oxygen demand [5], we propose that a change in energetics could be yet another mechanism that dictates distinct gene-expression programs in chronic overload.

\section{Mechanical phenotyping: perspectives and clinical implications}

Patients with hypertrophy are well known to be at increased risk for fatal arrhythmias and prone to develop overt heart failure. The basic causes for electrical dysfunction and contractile deterioration in hypertrophy are still unclear and at the molecular-functional level they vary with the type of overload and the genetic coping mechanisms of the affected individual [13]. Mechanistic understanding could be significantly improved by mechanical phenotyping of the chronically overloaded heart, as we have done in this study, to 1) determine the precise nature and cyclic occurrence of mechanical stimuli, 2) distinguish between the contribution of mechanical and other stimuli of remodeling, 3) monitor the transition from compensated to decompensated circulatory function, or 4) evaluate the effects of interventions. Our approach combines transthoracic echocardiography, invasive LV-pressure recordings and mathematical modeling, which is feasible in anesthetized experimental animals and potentially also in conscious patients. In the analysis of myocardial mechanics, we consider the LV to be thick-walled with anisotropic material containing myofibers. As a unique consequence, our levels of stress and strain are comparable to direct measurements in isolated cardiac muscle [3].

The incremental values of the present approach are 1) its minimally invasive character allowing serial intraindividual mechanical phenotyping at the tissue level, 2) the precise physical quantification of tissue mechanics, acting as an in vivo stress/strain 'measuring' setup that avoids myocardial damage, and 3) the simplicity of the model requiring just a few global LV quantities.

Others have applied three-dimensional motion reconstructions using tagged magnetic resonance imaging (MRI) to estimate stress and strain in vivo [12], or used ultrasound strain-rate imaging as a validated alternative for MRI-based methods in order to quantify myocardial deformation throughout the cardiac cycle [10]. These valuable techniques have so far only been used in a limited number of patients to serially estimate myocardial mechanics in chronic cardiac pathology $[19,31]$ and, like our approach, await application in larger populations.

\section{Limitations}

All experiments were conducted under general anesthesia to allow for stable, reproducible conditions. Anesthetic regimens included pentobarbital, halothane and nitrous oxide, which have been reported to depress contractile performance $[29,33]$. While this could have influenced our results to some extent, the serial analyses in individual dogs before and after AVB would still permit an accurate assessment of relative mechanical changes. 
We demonstrated that myofiber mechanics and LV mass underwent considerable changes already within the first 2 weeks of AVB. Preferably, future studies should include more time-points of analysis during this early phase, but the number of experiments (requiring anesthesia) within such a short period is limited for ethical reasons.

\section{Conclusions}

Serial mechanical phenotyping at the tissue level is practicable in the intact heart of anesthetized dogs to monitor the time-course of chronic overload. In dogs with AVB, increased end-diastolic myofiber stress $\left(\sigma_{\mathrm{f}}\right)$, and ejection strain $\left(\Delta \mathrm{e}_{\mathrm{f}, \mathrm{j}}\right)$ during systole are the most prominent mechanical changes, resulting in the elevation of myofiber stroke work. Early changes of these local mechanics coincide with peak hypertrophic growth, suggesting their important role for mechanotransduction. Our new approach, which combines transthoracic echocardiography, invasive LV-pressure recordings and mathematical modeling of myofiber mechanics allows a direct comparison of values for $\sigma_{f}$ and $e_{f}$ with those obtained in experiments on isolated cardiac muscle. This proper quantification may prove useful to further enhance understanding of mechanotransduction in the beating heart, and has potential clinical implications as an individualized approach to diagnosis and tailoring of therapy. 


\section{APPENDIX}

\section{LV cavity and wall volume}

For the calculation of cavity and wall volume, the LV is assumed to be a truncated ellipsoid. LV long axis inner diameter $\left(\mathrm{l}_{\mathrm{ba}}\right)$ is herein a $75 \%$ truncation of the longitudinal length of the ellipsoid. Outer LV short-axis diameter $\left(d_{0}\right)$ was calculated as the sum of the inner diameter $\left(d_{i}\right)$, posterior and septal wall thickness. Inner and outer volumes $\left(V_{i^{\prime}}, V_{0}\right)$ depend on inner and outer short-axis diameters $\left(d_{i}\right.$, $d_{0}$ ) and length $I_{b a}$ by:

$$
\begin{aligned}
& V_{i}=\left(\frac{3 \pi}{16}\right) I_{b a} d_{i}^{2} \\
& V_{o}=\left(\frac{3 \pi}{16}\right)\left(I_{b a}+\frac{3}{2 h_{a W}}\right) d_{o}^{2}
\end{aligned}
$$

The variable $h_{a w}$ represents wall thickness at the apex. Using that for the reference state (subscript 'ref'), local wall thickness is proportional to wall tension (T) [1]. Apical wall thickness $h_{\text {aw,ref }}$ is estimated as a fraction of equatorial wall thickness, depending on ellipsoid eccentricity E. (See also below: 'Estimation of apical wall thickness'.)

With

$$
h_{a w, r e f}=\left(d_{o, r e f}-d_{i, r e f}\right) \frac{E}{3 E^{2}-1}
$$

$$
E=\frac{(8 / 3) I_{b a}}{d_{o, \text { ref }}+d_{i, \text { ref }}}
$$

Reference diameters $\left(d_{o, \text { ref' }} d_{i, r e f}\right)$ are obtained by averaging diameters $\left(d_{0}, d_{i}\right)$ over all systolic values. Per measuring moment the ratio $\mathrm{I}_{\mathrm{ba}} /\left(\mathrm{d}_{\mathrm{i}, \text { ref }}+\mathrm{d}_{\mathrm{o}, \text { ref }}\right)$ is averaged over the group of measurements. Length $\mathrm{I}_{\mathrm{ba}}$ for a given measurement is obtained by multiplication of this averaged ratio with $\left(\mathrm{d}_{\mathrm{i}, \text { ref }}+\mathrm{d}_{\mathrm{o}, \text { ref }}\right)$ of the particular experiment. Substituting the result of Eq. 2 in Eq. 1, wall volume $V_{w}$ is (by subtraction of inner from outer volume in the reference state):

$$
V_{w}=V_{o, r e f}-V_{i, r e f}
$$

For calculation of the dynamically varying $L V$ volume $V_{L V^{\prime}}$ dynamically varying $h_{a w}$ is corrected for wall thinning with diameter increase:

$$
h_{a w}=h_{a W, \text { ref }} \frac{\left(d_{o, r e f}+d_{i, r e f}\right)^{2}}{\left(d_{o}+d_{i}\right)^{2}}
$$

Thus, $V_{L V}$ is expressed as a function of $h_{a W^{\prime}} d_{0}$ and $d_{i^{\prime}}$ while using Eqs. 1,3: 


$$
V_{L V}=\frac{\left(V_{o}-V_{W}+V_{i}\right)}{2}
$$

Eq. 5

The use of $V_{L V}$ is preferred over $V_{i}$ because of efficient noise reduction by appropriate averaging.

\section{Estimation of apical wall thickness}

Wall thickness is considered to be proportional with total wall tension $T$, being the sum of two perpendicular components. Assuming a thin-walled ellipsoid with long to short-axis ratio $E_{1}$ the radius of curvature at equator $\left(\rho_{\mathrm{ez}}\right)$ and apex $\left(\rho_{\mathrm{a}}\right)$ depends on short-axis length $2 r$ and $E$ by:

$$
\rho_{e z}=r E^{2} \text { and } \rho_{a}=\frac{r}{E}
$$

Eq. 6

Using Laplace's law on membrane tension, for equator and apex it holds that

$$
p_{L V}=\frac{T_{e c}}{r}+\frac{T_{e z}}{\rho_{e z}} \text { and } p_{L V}=\frac{2 T_{a}}{\rho_{a}}
$$

Subscripts 'a', 'ec', and 'ez' refer to apical, equatorial-circumferential, and equatorial-axial, respectively. For axial tension at the equator $\left(\mathrm{T}_{\mathrm{ez}}\right)$ it holds, like in a pressurized cylinder:

$$
T_{e z}=p_{L V} \frac{r}{2}
$$

Eq. 8

Solving Eqs. 6-8 by expressing tensions as a function of $\rho_{\mathrm{LV}}, \mathrm{r}$ and $\mathrm{E}$, for the ratio of wall thickness at apex to that of base, it is found:

$$
\frac{h_{a w}}{h_{e}}=\frac{2 T_{a}}{T_{e c}+T_{e z}}=\frac{2 E}{3 E^{2}-1}
$$

Eq. 9 has been applied to obtain Eq. 2 .

\section{Acknowledgments}

P.G.A.V. is supported by The Netherlands Organization for Health Research and Development (ZonMw 906-02-068). The authors wish to thank Iwan de Jong, BS, IDEE, Maastricht University, and Theo van der Nagel, Department of Cardio-Thoracic Surgery, Cardiovascular Research Institute Maastricht, Academic Hospital Maastricht, Netherlands, for technical assistance. 


\section{REFERENCES}

1. Arts T, Bovendeerd PH, Prinzen FW et al. (1991) Relation between left ventricular cavity pressure and volume and systolic fiber stress and strain in the wall. Biophys J 59:93-102

2. Ashikaga H, Covell JW, Omens JH (2005) Diastolic dysfunction in volume overload hypertrophy is associated with abnormal shearing of myolaminar sheets. Am J Physiol Heart Circ Physiol (Epub ahead of print)

3. de Tombe PP, ter Keurs HE (1991) Sarcomere dynamics in cat cardiac trabeculae. Circ Res 68:588-596

4. Delhaas T, Arts T, Bovendeerd PH et al. (1993) Subepicardial fiber strain and stress as related to left ventricular pressure and volume. Am J Physiol 264:H1548-H1559

5. Delhaas T, Arts T, Prinzen FW et al. (1998) Estimates of regional work in the canine left ventricle. Prog Biophys Mol Biol 69:273-287

6. Falsetti HL, Mates RE, Grant C et al. (1970) Left ventricular wall stress calculated from one-plane cineangiography. Circ Res 26:71-83

7. Florenzano F, Glantz SA (1987) Left ventricular mechanical adaptation to chronic aortic regurgitation in intact dogs. Am J Physiol 252:H969-H984

8. Granzier HL, Irving TC (1995) Passive tension in cardiac muscle: contribution of collagen, titin, microtubules, and intermediate filaments. Biophys J 68:1027-1044

9. Grossman W, Jones D, McLaurin LP (1975) Wall stress and patterns of hypertrophy in the human left ventricle. J Clin Invest 56:56-64

10. Herbots L, Maes F, D'Hooge J et al. (2004) Quantifying myocardial deformation throughout the cardiac cycle: a comparison of ultrasound strain rate, grey-scale $\mathrm{M}$-mode and magnetic resonance imaging. Ultrasound Med Biol 30:591-598

11. Holmes JW (2004) Candidate mechanical stimuli for hypertrophy during volume overload. J Appl Physiol 97:1453-1460

12. Hu Z, Metaxas D, Axel L (2003) In vivo strain and stress estimation of the heart left and right ventricles from MRI images. Med Image Anal 7:435-444

13. Hunter JJ, Chien KR (1999) Signaling pathways for cardiac hypertrophy and failure. N Engl J Med 341:1276-1283

14. Kerckhoffs RC, Bovendeerd PH, Kotte JC et al. (2003) Homogeneity of cardiac contraction despite physiological asynchrony of depolarization: a model study. Ann Biomed Eng 31:536-547

15. Komuro I, Kaida T, Shibazaki Y et al. (1990) Stretching cardiac myocytes stimulates protooncogene expression. J Biol Chem 265:3595-3598

16. Mirsky I (1973) Ventricular and arterial wall stresses based on large deformation analyses. Biophys J 13:1141-1159

17. Miyamoto T, Takeishi Y, Takahashi H et al. (2004) Activation of distinct signal transduction pathways in hypertrophied hearts by pressure and volume overload. Basic Res Cardiol 99:328-337

18. Modesti PA, Vanni S, Bertolozzi I et al. (2000) Early sequence of cardiac adaptations and growth factor formation in pressure- and volume-overload hypertrophy. Am J Physiol Heart Circ Physiol 279:H976-H985

19. Moustakidis P, Cupps BP, Pomerantz BJ et al. (2004) Noninvasive, quantitative assessment of left ventricular function in ischemic cardiomyopathy. J Surg Res 116:187-196 
20. Nomura S (1986) Diastolic property of left ventricle under experimental volume overload. Jpn Circ J 50:426-432

21. Omens JH (1998) Stress and strain as regulators of myocardial growth. Prog Biophys Mol Biol 69:559-572

22. Omens JH, Covell JW (1991) Transmural distribution of myocardial tissue growth induced by volumeoverload hypertrophy in the dog. Circulation 84:1235-1245

23. Reichek N, Helak J, Plappert T et al. (1983) Anatomic validation of left ventricular mass estimates from clinical two-dimensional echocardiography: initial results. Circulation 67:348-352

24. Sadoshima J, Izumo S (1997) The cellular and molecular response of cardiac myocytes to mechanical stress. Annu Rev Physiol 59:551-571

25. Schreiner KD, Voss F, Senges JC et al. (2004) Tridimensional activation patterns of acquired torsade-depointestachycardias in dogs with chronic AV-block. Basic Res Cardiol 99:288-298

26. Serneri GG, Modesti PA, Boddi M et al. (1999) Cardiac growth factors in human hypertrophy. Relations with myocardial contractility and wall stress. Circ Res 85:57-67

27. Simpson DG, Majeski M, Borg TK et al. (1999) Regulation of cardiac myocyte protein turnover and myo. brillar structure in vitro by specific directions of stretch. Circ Res 85:e59-e69

28. Sipido KR, Volders PG, de Groot SH et al. (2000) Enhanced $\mathrm{Ca}(2+)$ release and $\mathrm{Na} / \mathrm{Ca}$ exchange activity in hypertrophied canine ventricular myocytes: potential link between contractile adaptation and arrhythmogenesis. Circulation 102:2137-2144

29. Snyder DS, Harasawa Y, Sagawa K et al. (1993) Effects of pentobarbital on inotropic state of isolated canine left ventricle. Heart Vessels 8:128-135

30. Stevens C, Remme E, LeGrice I et al. (2003) Ventricular mechanics in diastole: material parameter sensitivity. J Biomech 36:737-748

31. Van der Toorn A, Barenbrug P, Snoep G et al. (2002) Transmural gradients of cardiac myofiber shortening in aortic valve stenosis patients using MRI tagging. Am J Physiol Heart Circ Physiol 283:H1609-1615

32. Van Opstal JM, Verduyn SC, Leunissen HD et al. (2001) Electrophysiological parameters indicative of sudden cardiac death in the dog with chronic complete AV-block. Cardiovasc Res 50:354-361

33. Van Trigt P, Christian CC, Fagraeus L et al. (1984) Myocardial depression by anesthetic agents (halothane, enflurane and nitrous oxide): quantitation based on end-systolic pressure-dimension relations. Am J Cardiol 53:243-247

34. Volders PG, Sipido KR, Vos MA et al. (1998) Cellular basis of biventricular hypertrophy and arrhythmogenesis in dogs with chronic complete atrioventricular block and acquired torsade de pointes. Circulation 98:1136-1147

35. Vos MA, de Groot SH, Verduyn SC et al. (1998) Enhanced susceptibility for acquired torsade de pointes arrhythmias in the dog with chronic, complete AV block is related to cardiac hypertrophy and electrical remodeling. Circulation 98:1125-1135

36. Watson PA, Hannan R, Carl LL et al. (1996) Desmin gene expression in cardiac myocytes is responsive to contractile activity and stretch. Am J Physiol 270:C1228-C1235

37. Wusten B, Flameng W, Winkler B et al. (1977) Role of cardiac contractility in hypertrophy from chronic volume loading. Cardiovasc Res 11:132-140

38. Yamamoto K, Dang QN, Maeda Y et al. (2001) Regulation of cardiomyocyte mechanotransduction by the cardiac cycle. Circulation 103:1459-1464 
Dirk W. Donker, Jos G. Maessen, Roel L.H.M.G. Spätjens, Theo van der Nagel, Monique de Jong, Frans C. Ramaekers, Harry J.G.M. Crijns, Marc A. Vos, Paul G.A. Volders.

Pflugers Arch. 2007;454:1043-51 


\section{Serial left-ventricular}

biopsy sampling using a

minimally invasive trans-thoracic approach in adult dogs 


\begin{abstract}
Myocardial biopsies are an increasingly important tool to unravel the molecular mechanisms of cardiac disease. We evaluate a novel minimally invasive transthoracic approach for left-ventricular (LV) intra-mural biopsies, which enables repetitive individual sampling in adult dogs. Forty three generally anaesthesised dogs were studied during sinus rhythm (SR, control) and multiple times after the induction of volume overload hypertrophy (complete atrioventricular block [AVB]). Through a small cutaneous incision, an automatic biopsy needle was advanced into the apicolateral LV, guided by fluoroscopy. Electrocardiography (ECG), LV pressure and echocardiography served to monitor the procedure. One hundred eighty-eight intra-mural LV biopsies were obtained in 82 serial experiments (usually SR, 1, 2 and 6 weeks AVB) with a maximum of 8 repeated biopsies. All biopsies $\left(\sim 10 \mathrm{~mm}^{3}\right)$ were suitable for simultaneous application of different cell-biological (light and electron microscopy, immunohistochemistry) and molecular techniques (PCR, Western blotting). In chronic experiments, repeated biopsy sampling did not influence hemodynamics, mechanics, electrocardiographic parameters or myocardial remodelling during SR or AVB. The rate of significant complications was as low as $4 \%$ of experiments. Minimally invasive sampling of LV needle biopsies enables serial assessment of myocardial remodelling using different molecular techniques in individual animals. The technique is safe and has no long-term effects on cardiac function or structure.
\end{abstract}




\section{INTRODUCTION}

Myocardial biopsy sampling offers indispensable diagnostic options to unravel the mechanisms of myocardial disease in different conditions $[3,18]$. Currently, cardiac biopsies are mostly obtained using a trans-venous catheter-based bioptome enabling to sample sub-endomyocardial tissue from the right ventricle (RV), as introduced in 1962 [13]. This trans-vascular approach is also used trans-arterially to sample from the left ventricle (LV) [9]. In experienced hands, the catheter-based technique is relatively safe with complication rates of 1-4\% [10] and is routinely applied in clinical referral centers [9]. It is important to note that the limited invasiveness of the technique makes it possible to sample cardiac biopsies in a serial manner to monitor specific disease processes in individual patients over time. Yet, (sub)endomyocardial tissue samples have significant drawbacks relating to (1) sampling errors [15] and lack of representativity, which requires to take at least 5 biopsies per session and even more for focal processes [19]; (2) small sample size (usually $<1 \mathrm{~mm}^{3}$ ) [15] and (3) poor tissue quality due to squeezing and cutting artefacts in a relatively small tissue sample [11], which often render the biopsies unsuitable for histology, immunohistochemistry and electron microscopy [15].

An alternative way to sample ventricular myocardial tissue is by the use of a biopsy needle, which enables to obtain larger, intra-mural samples of superior quality compared to endomyocardial biopsies. This technique was first described using limited thoracotomy [7], but needle biopsies have generally been confined to the use in patients and animals undergoing standard thoracotomy for direct vision of the puncture site and potential bleeding [17]. Today, needle biopsies can be safely and quickly obtained during open-heart surgery using automatic biopsy devices $[14,20]$.

In the study reported here, we developed a novel transthoracic approach to sample LV needle biopsies from individual anaesthetised dogs in a serial manner and evaluated its application in chronic experiments after the induction of cardiac hypertrophy.

\section{MATERIALS AND METHODS}

Experiments were conducted in accordance with the 'European Directive for the Protection of Vertebrate Animals Used for Experimental and Other Scientific Purposes (86/609/ EU)'. The Committee for Experiments on Animals of Maastricht University approved the experiments.

\section{Serial trans-thoracic sampling of LV intra-mural biopsies}

We studied a total number of 43 adult mongrel dogs of either gender weighing $28 \pm 1 \mathrm{~kg}$ at baseline during normal sinus rhythm (SR, control). All experiments were performed under general anaesthesia. After overnight fasting, pre-medication $(0.15 \mathrm{mg} / \mathrm{kg}$ acepromazine, $0.4 \mathrm{mg} / \mathrm{kg}$ methadone, 
$0.06 \mathrm{mg} / \mathrm{kg}$ atropine i.m.) was administered. Complete anaesthesia was induced by thiopental (20 $\mathrm{mg} / \mathrm{kg}$ i.v.) and maintained with halothane (0.5-1\%) and $\mathrm{O}_{2}: \mathrm{N}_{2} \mathrm{O}(1: 2)$.

The dogs were positioned in a right-sided supine position, the left anterolateral thorax was shaved, disinfected with iodine and draped with sterile towels. The apex was palpated on the left-sided chest. The X-ray tube was set to a vertical position $\left(0^{\circ}\right)$ to identify the apicolateral LV in the sagittal plane (Figure 1a), and visualised in an oblique left-lateral fluoroscopic view (Figure 1b). In the sagittal plane, the biopsy needle forms an angle of $\sim 60^{\circ}$ with the longitudinal axis of the sternum. In the transverse plane (Figure 1c), the apicolateral LV was approached by holding the biopsy needle in an angle of $\sim 60^{\circ}$ to an imaginary horizontal line, in parallel to the table (Figure 1c). Mechanical ventilation was briefly stopped during sampling to minimise the risk of pulmonary injury. A $14 \mathrm{G}$ automatic biopsy needle (Acecut, TSK Laboratory, Japan) was opened and slowly advanced trans-thoracically through a small $(\sim 5 \mathrm{~mm})$ cutaneous incision in the inter-costal space overlying the LV apex (Figure 1d). This was done under continuous fluoroscopic guidance whilst approaching the apicolateral LV wall (Figure 1b). Contact with the epicardial surface was recognised by sensing a mild resistance and cardiac pulsations as pulse-synchronous motion of the needle shaft, often accompanied by ventricular extra-systoles on the electrocardiogram (ECG) (Figure 2a). Subsequently, the needle was quickly advanced into the epicardial layers at an angle of $\sim 60^{\circ}$ to the surface. Then, it was forwarded by $\sim 10 \mathrm{~mm}$ as controlled by centimetre markers on the shaft to assure deep intra-mural sampling. The oblique angle to the epicardial surface was applied to minimise the risk of hemopericardium due to trans-mural puncture. After puncture, the automatic cutting mechanism of the biopsy needle was immediately released and the device was retracted. We aimed at completing the intra-myocardial insertion of the needle, the actual sampling and the retraction of the device within 2-3 heartbeats to avoid unnecessary movements of the device, potentially causing additional damage or bleeding of the myocardium or arrhythmias. At least two cylindrical transmural samples were taken per experiment. For further processing, biopsies were either immediately frozen in isopentane, pre-cooled with liquid nitrogen and stored at $-80^{\circ} \mathrm{C}$ or fixed in $3 \%$ glutaraldehyde and stored at $4^{\circ} \mathrm{C}$. Light and electron microscopy, PCR and Western blotting were performed according to standard protocols $[4,16]$.

Throughout the procedure, a standard 6-lead ECG was registered and the LV intra-cavitary blood pressure was monitored in a sub-set of experiments (Figure 2a and Figure 2b). After sampling, all animals were followed-up echocardiographically (Figure 2c) for at least 60 min to monitor LV contractility and potential occurrence of pericardial effusion. Echocardiograms were usually performed every 5 min during the first 20 min after sampling. If pericardial effusion was absent or had not increased to a maximum of more than $\sim 5 \mathrm{~mm}$, anaesthesia was terminated. After detubation, additional echocardiograms were performed before and after transportation to the cage, usually at 30, 45 and 60 min after sampling. When pericardial effusion increased to more than $\sim 10 \mathrm{~mm}$ in the first hour after sampling, we decided to intervene surgically by left-lateral thoracotomy, pericardiotomy, evacuation of blood from the pericardial space and local hemostasis 
a

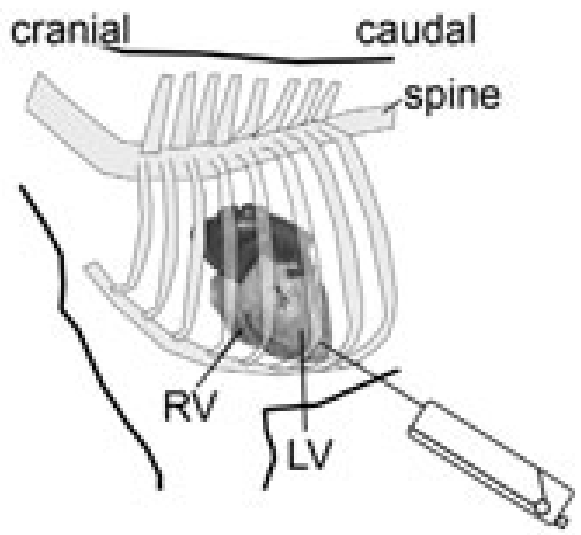

C

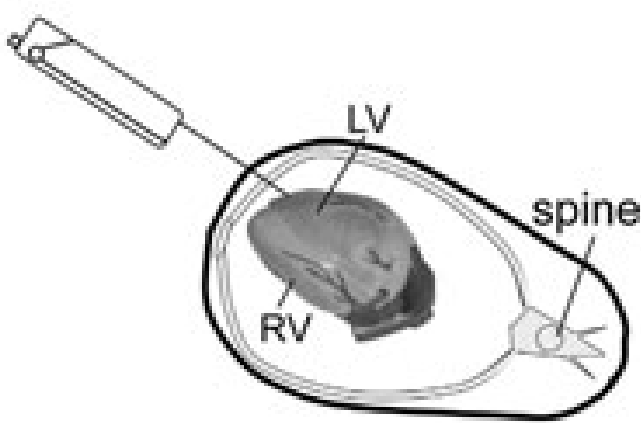

b

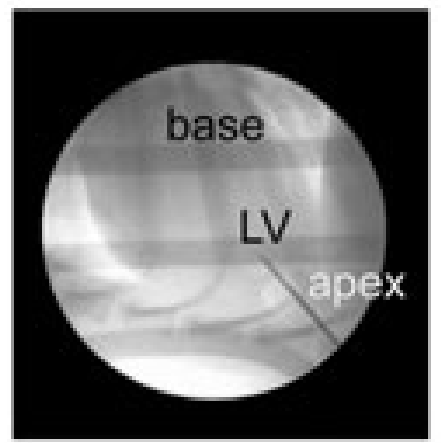

Q

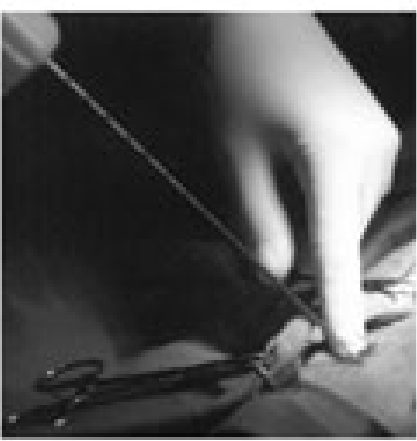

Figure 1 | Serial percutaneous sampling of LV intra-mural biopsies. a Schematic illustration of optimal biopsy needle positioning in the sagittal plane in relation to the intra-thoracic location of the heart in the adult dog. $\mathbf{b}$ Under fluoroscopic guidance, the needle is advanced into the apicolateral LV wall. c Schematic illustration of optimal biopsy needle positioning in the transverse plane (caudal view) in relation to the intra-thoracic location of the heart. $\mathbf{d}$ A small incision facilitates percutaneous introduction of the biopsy needle into the left anterolateral inter-costal space overlying the LV apex. See text for detailed description. For full colour illustration, see page 222. 

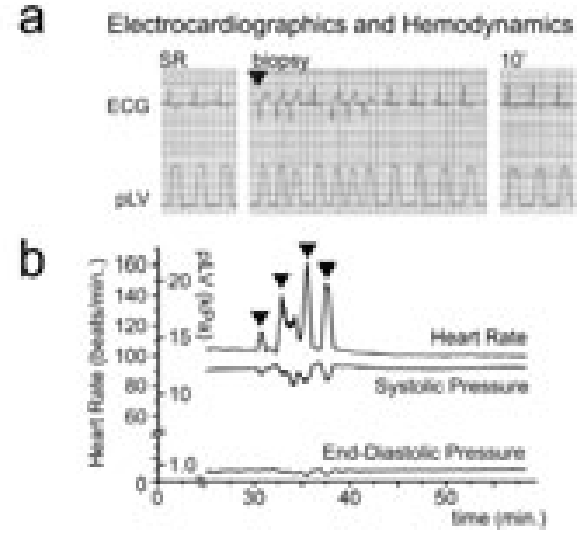
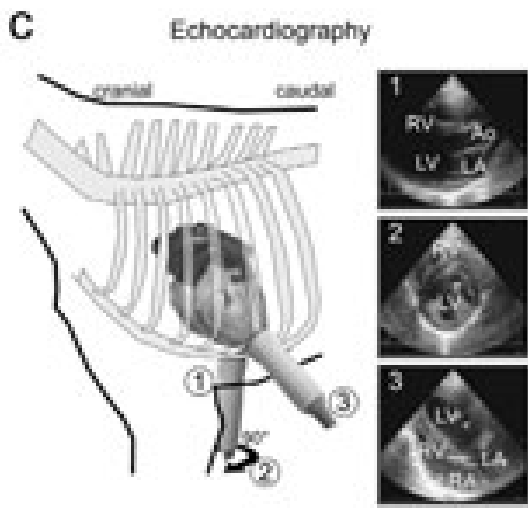

Figure 2 Procedural monitoring during biopsy sampling. a Electrocardiographic and hemodynamic monitoring shows ventricular extrasystoles (arrowhead) during intra-myocardial insertion of the biopsy needle. SR, sinus rhythm, pLV, intra-cavitary LV pressure. Ten minutes after sampling, the ECG and LV pressure tracings are similar to the baseline (SR). $\mathbf{b}$ Monitoring of heart rate and LV systolic and diastolic pressure trends reveals stable tracings after sequential sampling of four biopsies accompanied by multiple ventricular extra-systoles (arrowheads). c Trans-thoracic echocardiographic approach in the adult dog. The right parasternal transducer positions allows long axis (1) and, after rotation, short axis views (2), whereas left parasternal apical scanning provides four-chamber views (3). Note that besides B-mode also M-mode and Doppler-flow measurements (not shown) can be easily performed. Ao, ascending aorta; LA, left atrium; RA, right atrium. For full colour illustration, see page 223.

of the bleeding site. LV hemodynamics, mechanics and echocardiographic estimates of LV mass were assessed as described before $[4,5]$. The complete procedure is described as a practical stepby-step approach in Flowchart 1.

All dogs were studied serially during SR and after the induction of complete atrioventricular block (AVB). AVB was induced by radiofrequency (RF) catheter ablation of the His bundle. Sampling was performed under baseline conditions at sinus rhythm (SR; control) and, in the same animals, at different time points after AVB ranging from 2 days up to 6 weeks. The dogs were sacrificed several weeks later and the hearts were excised and inspected macroscopically.

\section{STATISTICS}

Data are presented as the mean \pm standard error of the mean (SEM). Data were compared using Student's t-test for unpaired data. Differences were considered statistically significant if $\mathrm{P}<0.05$. 


\section{RESULTS}

The biopsies measured $\sim 1 \times 1 \times 10 \mathrm{~mm}$ and weighed $\sim 15 \mathrm{mg}$ (Figure 3a). The tissue was of good quality and suitable for subsequent analysis in all biopsies. To assess the intra-mural extension of the biopsies, we performed light-microscopic analyses of complete specimens (Figure 3b) and (sub) endocardial pieces (Figure 3c), demonstrating that (sub)epicardial and (sub)endocardial myocardium is present in, at least, a subset of the biopsies. Macroscopic inspection of the sampling sites revealed proper sampling from the apicolateral LV wall and only minimal local fibrosis with a diameter of $\sim 1-2 \mathrm{~mm}$ limited to the puncture site (Figure 3d).

Light and electron microscopy revealed a normal homogeneous myocardial texture in the absence of contraction bands allowing detailed analyses of the cardiomyocyte (ultra) structure and of cell-cell contacts (Figure $4 a$ and Figure $4 b$ ). Representative immunohistochemical double labelling allows to study intra-myocardial protein localisation and their spatial distribution pattern in cardiomyocytes, noncardiomyocytes and extra-cellular matrix (Figure 4c).

\section{Serial LV function and structure in bioptized vs non-bioptized dogs}

Serial analysis of LV systolic and diastolic hemodynamics, mechanics and LV mass increase in chronic experiments revealed no significant differences between repeatedly bioptized and non-bioptized individual dogs after induction of bradycardia-induced volume overload due to AVB (Figure 5). Also, electrocardiographic parameters were comparable in both groups over time (Figure 6).

To control for influences of repeated biopsy sampling itself on protein expression, we studied cardiomyocytespecific proteins known to change after AVB, such as the $\mathrm{K}^{+}$channel sub-unit KCNQ1 and the $B-1$ adrenergic receptor [16]. The expression level of both proteins remained unaltered in repeated biopsies of three control dogs (Figure 7).

\section{Procedural experiences and complications}

Generally, the animals recovered from the procedure within a few hours and post-operative wound infection was not observed. In the first 34 experiments, we observed cardiac tamponade in $5(15 \%)$ experiments. Four dogs died (12\%) and 1 underwent successful left-lateral thoracotomy, pericardiotomy and operative evacuation of the hemopericardium. It is important to note that all these dogs were heparinized (1,000 IU i.v.) around biopsy sampling, as routinely applied in our operation room to avoid catheter-induced thromboembolism and only 3 dogs had been hemodynamically monitored during the experiment.

To reduce the initial complication rate, we adapted the procedure: heparin was omitted without negative sequelae, and echocardiography was introduced to monitor the procedure, especially the occurrence of hemopericardium. The latter occurred in only $2(4 \%)$ of 48 experiments and could be immediately diagnosed and treated surgically in 1 dog having tamponade, whereas the other dog 

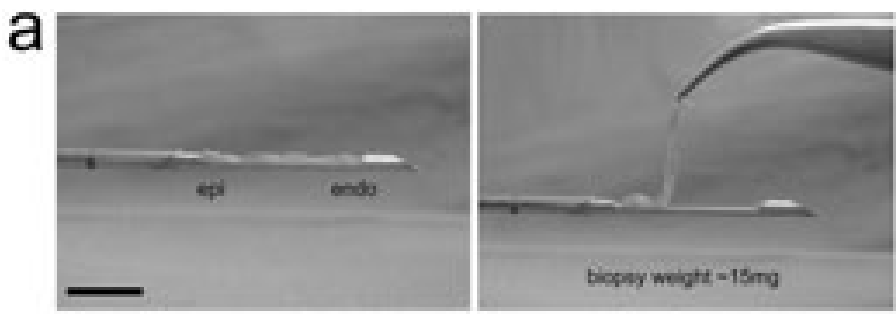

\section{(cryo)sections}
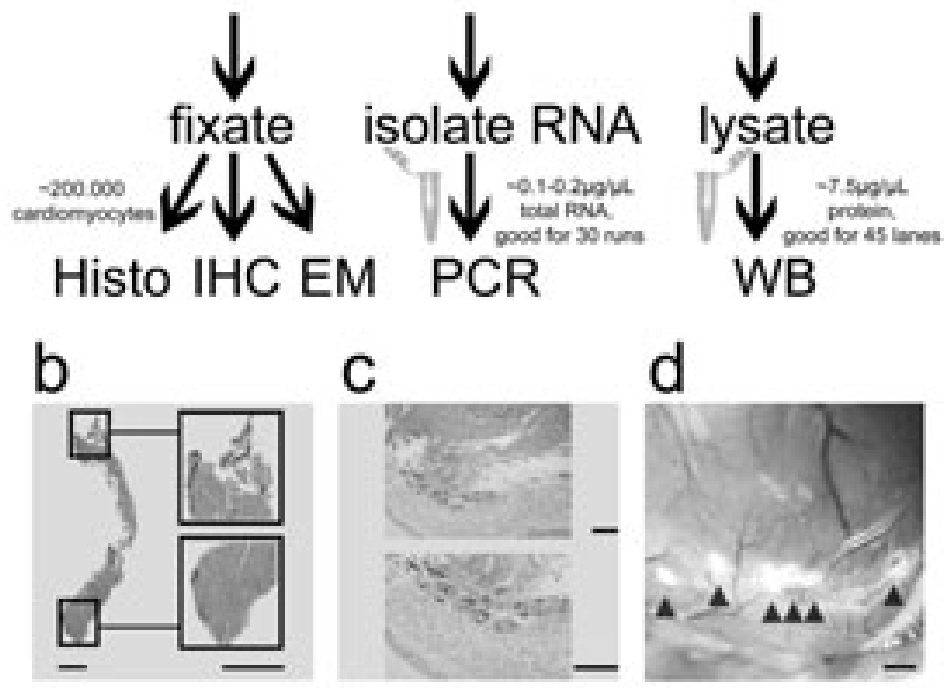

Figure $3 \mid$ Processing of LV myocardial needle biopsies. a Biopsies can be easily lifted from the needle with tweezers. The specimen can be processed as a complete intra-mural cylinder or can be dissected into smaller pieces. After freezing, cryosections can be prepared, allowing simultaneous application of different analytical methods as e.g., Histo histology, IHC immunohistochemistry, EM electron microscopy, PCR polymerase chain reaction and WB Western blotting. Scale bar indicates $1 \mathrm{~cm}$. b The LM photomicrograph illustrates the good quality of the myocardial samples using Masson's Trichrome staining (cardiomyocytes [red]/fibrous tissue [blue]). This example of an intra-mural biopsy extends from the epicardium (top inset) to the endocardium (bottom inset). Scale bars indicate $1 \mathrm{~mm}$. c Intra-mural extension of the biopsy sample can also be confirmed by visualisation of the (sub)endocardial layers as illustrated in the light-microscopic photograph using toluidine blue counterstained with periodic acid-Schiff; endocardium (pale blue), working myocardium (blue) and glycogen (magenta)containing Purkinje fibres. Scale bars indicate $100 \mu \mathrm{m}$. d At autopsy, only limited scar formation occurred at the sampling sites (arrowheads) after repeated biopsies. Scale bar indicates $2 \mathrm{~mm}$. For full colour illustration, see page 224. 
CHAPTER 4 | SERIAL LEFT-VENTRICULAR BIOPSY SAMPLING
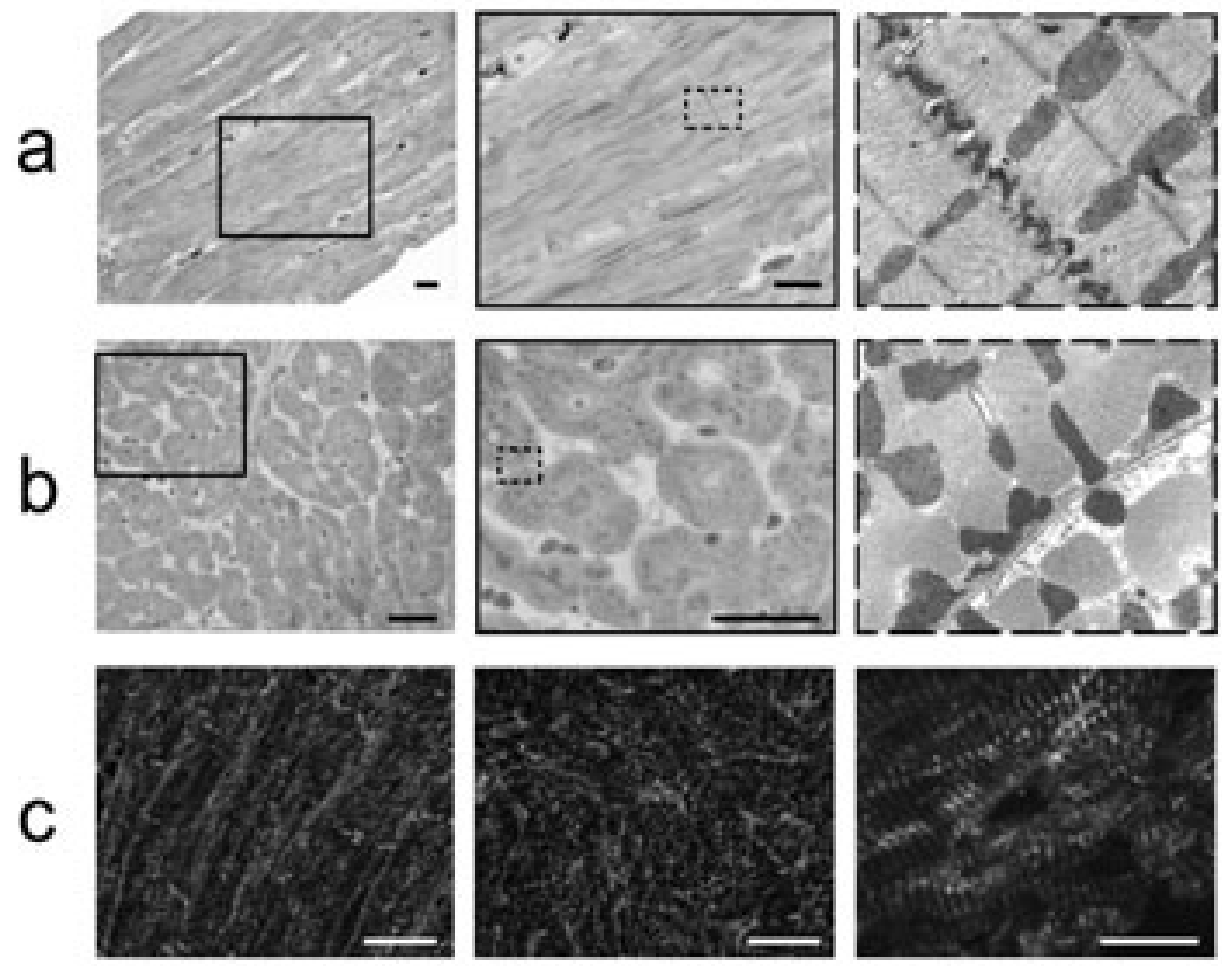

Figure $4 \mid$ Cell-biological myocardial analysis in LV needle biopsies. a Representative light-microscopic (toluidine blue counterstained with periodic acid Schiff) and electron-microscopic photographs showing longitudinally sectioned cardiomyocytes within a normal myocardial texture from a dog bioptized during SR, demonstrating the excellent tissue quality. Scale bars indicate $20 \mu \mathrm{m}$. b Light-microscopic and electron-microscopic photographs corresponding to a showing cross-sectioned cardiomyocytes. Scale bars indicate $20 \mu \mathrm{m}$. c Immunofluorescent histochemistry in LV myocardium double-labelling the cardiomyocyte intermediate filament desmin (green), the fibroblast intermediate filament vimentin (red; left and middle) and alpha smooth muscle actin present in the vessel wall (red, right), in longitudinally (left, right) and transversally sectioned myocardium (middle). Scale bars indicate $20 \mu \mathrm{m}$. For full colour illustration, see page 225. 

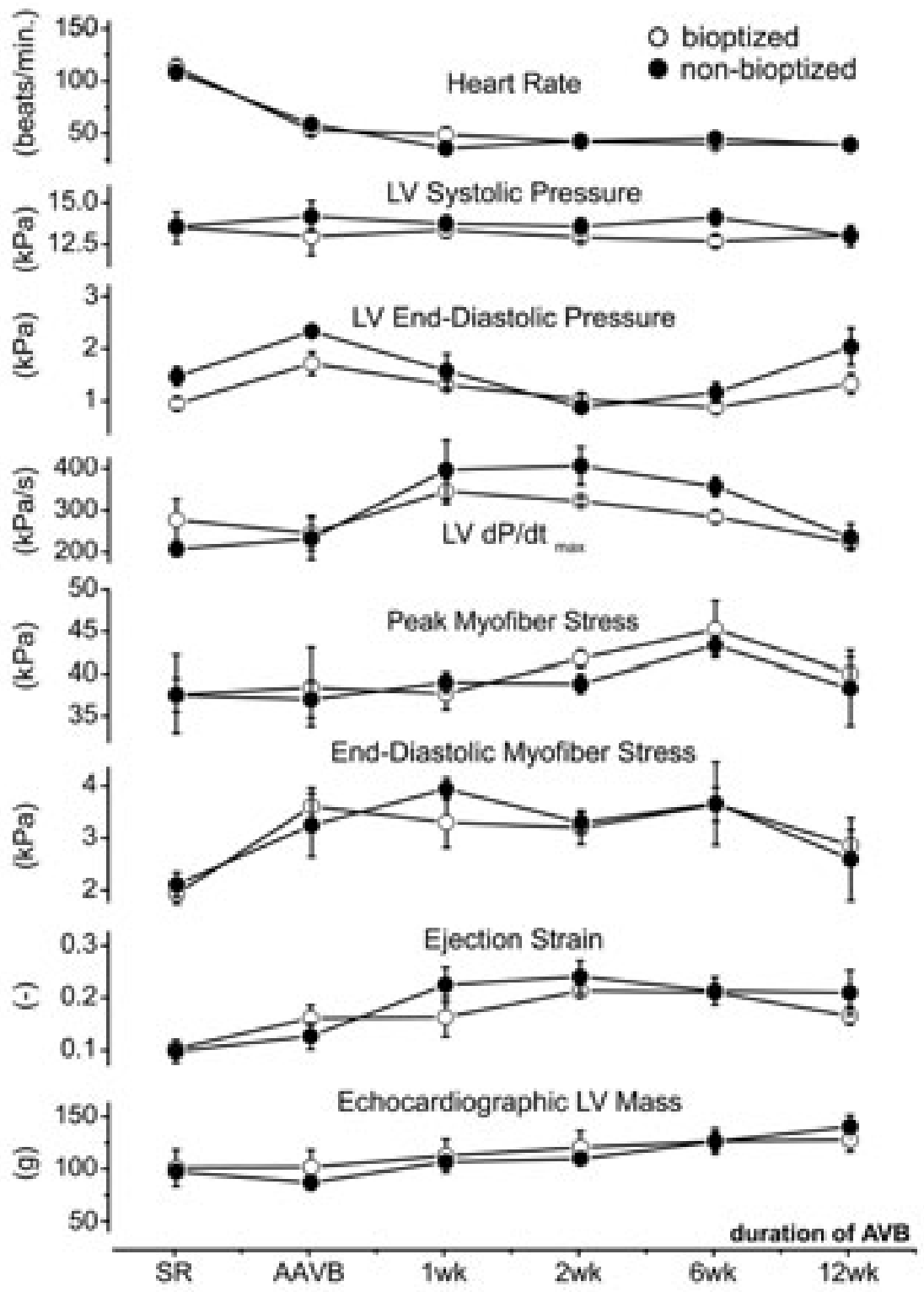

Figure $\mathbf{5} \mid$ Serial analysis of LV hemodynamics, mechanics and hypertrophy in bioptized vs non-bioptized animals. Group mean values of 4 dogs bioptized at SR, 1, 2 and 6 weeks AVB compared to 4 non-bioptized dogs at identical experimental timepoints. $P=n s$ for any parameter between the two groups. 

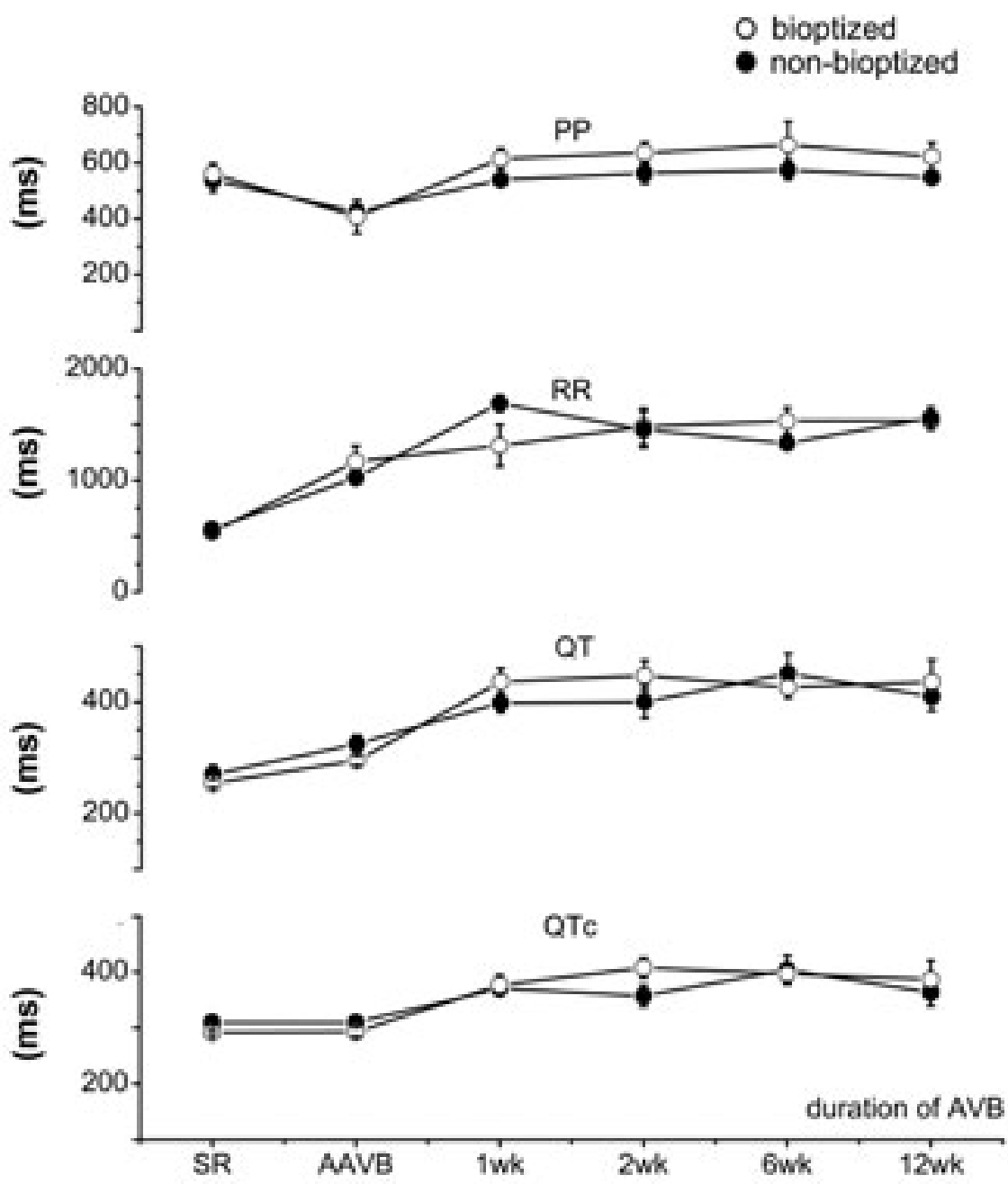

Figure 6 | Serial analysis of electrocardiographic parameters in bioptized vs non-bioptized animals. Group mean values of 4 dogs bioptized at SR, 1, 2 and 6 weeks AVB compared to 4 non-bioptized dogs at similar experimental timepoints. $P=n$ for any parameter between the two groups. 

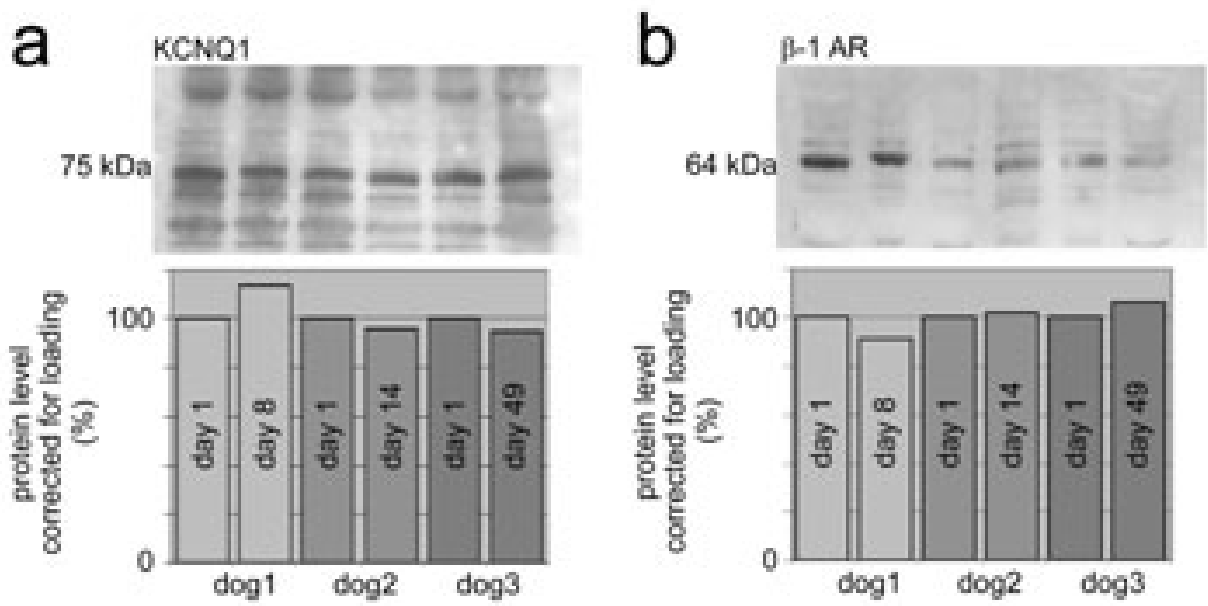

Figure 7 | Serial analysis of myocardial protein expression before and after biopsy sampling. a Western blot analysis shows that the $\mathrm{K}^{+}$-channel subunit KCNQ1 and $\mathbf{b}$ the $\mathrm{B}-1$ adrenergic receptor remained unaltered in repeated biopsies of control dogs in SR.

Table 1 Practical considerations

\begin{tabular}{|c|c|c|c|}
\hline Parameter & Technique & Recommendation & Complications / Comments \\
\hline $\begin{array}{l}\text { Identification of } \\
\text { sampling site }\end{array}$ & $\begin{array}{l}\text { Palpation/ } \\
\text { fluoroscopy }\end{array}$ & $\begin{array}{l}\text { Sample in apicolateral LV, avoid thin-walled } \\
\text { apex and coronaries in the lateral LV }\end{array}$ & Cardiac tamponade, coronary perforation \\
\hline \multirow[t]{3}{*}{$\begin{array}{l}\text { Intramyocardial } \\
\text { sampling }\end{array}$} & Operator & $\begin{array}{l}\text { Feel mild resistance and cardiac pulsations } \\
\text { on needle shaft before intra-myocardial } \\
\text { insertion }\end{array}$ & Extra-myocardial biopsy, no biopsy \\
\hline & ECG & $\begin{array}{l}\text { Monitor the occurrence of ventricular extra- } \\
\text { systoles originating from the biopsy site* }\end{array}$ & Extra-myocardial biopsy, no biopsy \\
\hline & Microscopy & $\begin{array}{l}\text { Use standard histological staining to ensure } \\
\text { tissue quality and intra-myocardial extent, } \\
\text { e.g., hematoxylin/eosin, toluidin blue, } \\
\text { Masson's trichrome }\end{array}$ & $\begin{array}{l}\text { Light microscopy allows to visualize } \\
\text { endocardium/epicardium and myocardium in } \\
\text { the same section }\end{array}$ \\
\hline \multirow[t]{3}{*}{ Cardiac function } & ECG & $\begin{array}{l}\text { Monitor standard leads throughout the } \\
\text { experiment }\end{array}$ & $\begin{array}{l}\text { Sinus tachycardia and signs of ischemia can } \\
\text { aid to diagnose a cardiac tamponade in time }\end{array}$ \\
\hline & Echocardiography & $\begin{array}{l}\text { Assess cardiac contractility and pericardial } \\
\text { effusion directly after sampling and repeat } \\
\text { frequently in the first hour }\end{array}$ & Cardiac tamponade can be diagnosed in time \\
\hline & $\begin{array}{l}\text { LV intra-cavitary } \\
\text { pressure catheter } \\
\text { (optional) }\end{array}$ & $\begin{array}{l}\text { Monitor LV systolic blood pressure } \\
\text { throughout the experiment (optional) }\end{array}$ & $\begin{array}{l}\text { Progressive decrease of systolic LV } \\
\text { blood pressure can aid to diagnose a cardiac } \\
\text { tamponade in time }\end{array}$ \\
\hline
\end{tabular}

${ }^{*}$ Arrhythmic ventricular responses may incidentally be absent. LV: left ventricle 
was found dead in the cage $>12 \mathrm{~h}$ after the experiment. Autopsy revealed a hemopericardium due to puncture of a small coronary side-branch. We summarized our experiences in Table 1.

\section{DISCUSSION}

In the present study, we demonstrate how LV needle biopsies can be repeatedly obtained in dogs undergoing chronic experiments. A trans-thoracic approach together with optimal safety monitoring ensures a low complication rate, quick post-operative recovery and optimal tissue quality. Serial myocardial sampling using needle biopsies in a chronic experimental setting is generally limited by the invasiveness of a thoracotomy, which results in only few sampling points, e.g., at the control stage and before sacrifice of the dogs [17]. We circumvented this by choosing a transthoracic, percutaneous approach, thus avoiding the thoracotomy. Historically, different closedchest approaches have been reported in canine and human hearts to sample ventricular needle biopsies from the LV anterior wall, apical area [2] and the inter-ventricular septum [1] under local or general anaesthesia. After the 1970s, percutaneous needle biopsy techniques have not been widely applied in patients or experimental animals, and no further technical developments have been reported. This might be largely due to the advent of catheter-based techniques, which are less invasive, relatively easy to use and safe, and, moreover, suitable for repeated biopsies.

The novel trans-thoracic approach we described in this study shares many advantages with the trans-vascular technique: (1) it is minimally invasive; (2) it has a low rate of potentially life-threatening complications $(\sim 4 \%)$ despite the limited number of experiments we performed, which is comparable to the clinically accepted trans-vascular techniques [10]; (3) it is suitable for repeated biopsies and thereby allows individual follow-up studies; (4) it can be performed within a few minutes; (5) it can be easily performed in a standard experimental animal laboratory (see Flowchart 1) with experienced bio-technicians reaching a sufficient level of competence after having performed $\sim 25$ biopsies. Moreover, the approach can likely be applied in other large animal species. This assumption is based on comparative anatomical considerations and reports of experiments with minimally invasive thoracoscopic cardiac access in sheep [6], pigs [21] and goats.

Multiple serial intra-mural biopsies from the same LV area did not influence functional and structural remodelling processes that occur after AVB. All analysed mechanical parameters were compatible with our earlier work $[4,5]$. Also, under control conditions, repeated biopsy sampling from the apicolateral LV wall did not significantly influence the expression of cardiomyocyte-specific proteins that have been shown to be altered in remodelling processes early after the induction of AVB [16]. These findings support the minimally invasive character of the technique and are in line with the macroscopic finding of only slight local fibrosis at barely visible puncture sites. Thus, it can be concluded that myocardial damage is confined to the puncture site. However, the total number of biopsies taken in individual animals might be relevant in this regard. We obtained a maximum 
of eight serial needle biopsies in up to four consecutive closed-chest experiments per dog. Higher numbers of serial ventricular needle biopsies in chronic experiments have only been reported in studies using cardiac allograft biopsies in dogs undergoing orthotopic [12] or heterotopic [8] heart transplantation. In these studies, post-operative fibrosis and adhesions after open-heart surgery likely play a role in reducing the risk of hemopericardium and tamponade, but may also increase the probability of biopsy-induced functional and structural myocardial changes.

Needle biopsies offer ample opportunities for a detailed myocardial analysis (Figure 3, Figure 4 and Figure 7). The tissue specimens are relatively large, cutting artefacts are limited to the border zones and they represent a cross-section through the ventricular wall potentially extending into the (sub) endocardium (Figure 3). Our serial approach allows to discern even mildly fluctuating expression patterns in long-term studies because individual dogs serve as their own controls $[4,16]$. These subtle changes are likely missed in group comparisons due to inherent inter-individual differences. These aspects meet contemporary issues in cardiac research, aimed at a comprehensive multi-level molecular analysis in individual subjects.

\section{CONCLUSIONS}

A novel minimally invasive trans-thoracic approach for serial LV intra-mural needle biopsies has been successfully developed in adult dogs. The procedure is safe and allows repeated sampling in individual animals without influencing cardiac function or structure in long-term experiments. Different cell-biological and molecular techniques can be simultaneously applied in these biopsy specimens. The approach is potentially applicable to other animal models of cardiac disease and offers the opportunity to study molecular mechanisms relevant for myocardial remodelling in chronic experiments relating to various pathological conditions.

\section{Acknowledgments}

This study was financially supported by the 'Stichting Hartsvrienden Rescar', Maastricht, The Netherlands. Dirk W. Donker was supported by Medtronic, The Netherlands. The authors wish to thank Jet Beekman (Dept. of Med. Physiol., Utrecht), Helma Kuijpers and Fons Verheyen (Dept. of Mol. Cell Biol., Maastricht) for technical assistance. 


\section{APPENDIX}

Flowchart 1 Procedure—minimally invasive biopsy technique

Induce general anesthesia, intubate and start mechanical ventilation

\begin{tabular}{|r|}
\hline Assure stable right-sided supine position of the animal * \\
\hline Shave and disinfect left-lateral thorax and assure sterile conditions \\
\hline Set X-ray tube to vertical position $\left(0^{\circ}\right)$ \\
\hline $\begin{array}{r}\text { Identify left-lateral thoracic area overlying apex } \\
\text { - Palpate apex pulsations (mark area) } \\
\text { - Adjust fluoroscopy to visualize apex (Figure } 1 \text { a, b) }\end{array}$ \\
\hline Make small ( 5 mm) cutaneous incision above apex \\
\hline
\end{tabular}

Find optimal spatial orientation of biopsy needle before insertion

- Sagittal plane: adjust needle at $\sim 60^{\circ}$ to sternum (Figure $1 \mathrm{a}, \mathrm{b}$ )

- Transverse plane: adjust needle at $\sim 60^{\circ}$ to table (Figure $1 \mathrm{c}, \mathrm{d}$ )

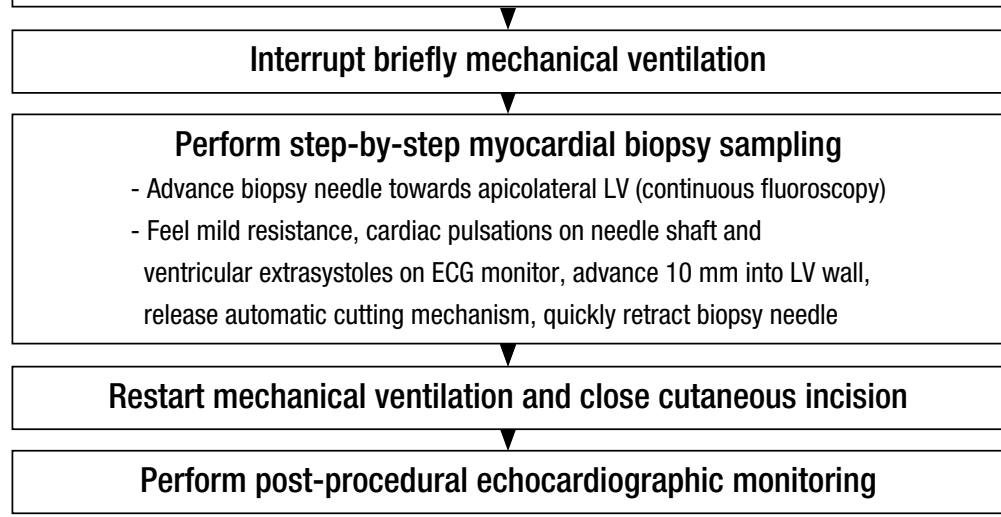

* Perform baseline echocardiograms for comparison to post-procedural echocardiograms 


\section{REFERENCES}

1. Bercu B, Heinz J, Choudhry AS, Cabrera P (1964) Myocardial biopsy. A new technic utilizing the ventricular septum. Am J Cardiol 14:675-678

2. Casten GG, Marsh JB (1953) Metabolic studies on cardiac tissue obtained by needle biopsy in the intact unanesthetized dog. Circ Res 1:226-229

3. Cunningham KS, Veinot JP, Butany J (2006) An approach to endomyocardial biopsy interpretation. J Clin Pathol 59:121-129

4. Donker DW, Maessen JG, Verheyen F, Ramaekers FC, Spätjens RLHMG, Kuijpers H, Ramakers C, Schiffers PMH, Vos MA, Crijns HJGM, Volders PGA (2007) Impact of acute and enduring volume overload on mechanotransduction and cytoskeletal integrity of canine left-ventricular myocardium. Am J Physiol Heart Circ Physiol 292:H2324-H2332 DOI 10.1152/ajpheart.00392.2006

5. Donker DW, Volders PGA, Arts T, Bekkers BCAM, Hofstra L, Spätjens RLHMG, Beekman JD, Borgers M, Crijns HJGM, Vos MA (2005) End-diastolic myofiber stress and ejection strain increase with ventricular volume overload-serial in-vivo analyses in dogs with complete atrioventricular block. Basic Res Cardiol 100:372-382

6. Joudinaud TM, Kegel CL, Gabster AA, Sanz ML, MacDonald A, Propp D, Callaghan E, Weber PA, Hvass U, Duran CM (2005) An experimental method for the percutaneous induction of a posterolateral infarct and functional ischemic mitral regurgitation. J Heart Valve Dis 14:460-466

7. Kent G, Sutton DC, Sutton GC (1956) Needle biopsy of the human ventricular myocardium. 0 Bull Northwest Univ Med Sch 30:213-214

8. Leedham PW, Baum M, Cullum PA (1971) Acute and modified rejection of heterotopic canine cardiac allotransplants studied by serial needle biopsy. Thorax 26:534-542

9. Mason JW (1978) Techniques for right and left ventricular endomyocardial biopsy. Am J Cardiol 41:887892

10. Mills AS, Hastillo A, Thompson JA, Hess ML (1985) Expectations and limitations of endomyocardial biopsy. Can J Cardiol 1:358-362

11. Olmesdahl PJ, Gregory MA, Cameron EW (1979) Ultrastructural artefacts in biopsied normal myocardium and their relevance to myocardial biopsy in man. Thorax 34:82-90

12. Penn OC, McDicken I, Bos E, Nauta J (1975) Serial percutaneous biopsies from canine orthotopic cardic allografts. J Surg Res 18:615-618

13. Sakakibara S, Konno S (1962) Endomyocardial biopsy. Jpn Heart J 3:537-543

14. Salerno TA, Lince DP, Wasan SM, Charrette EJ (1979) Intraoperative transmural myocardial biopsy: a simple technique. Can J Surg 22:487

15. Shu LG, de Geest H, Kesteloot H, van de Werf F, Aubert A, Borgers M, Janssen PA (1986) Closed chest myocardial biopsies: clinical uses and related problems. Acta Cardiol 41:79-87 
16. Stengl M, Ramakers C, Donker DW, Nabar A, Rybin AV, Spatjens RLHMG, van der Nagel T, Wodzig WKWH, Sipido KR, Antoons G, Moorman AF, Vos MA, Volders PGA (2006) Temporal patterns of electrical remodeling in canine ventricular hypertrophy: focus on IKs downregulation and blunted beta-adrenergic activation. Cardiovasc Res 72:90-100

17. Tagawa H, Koide M, Sato H, Zile MR, Carabello BA, Cooper G 4th (1998) Cytoskeletal role in the transition from compensated to decompensated hypertrophy during adult canine left ventricular pressure overloading. Circ Res 82:751-761

18. Veinot JP (2002) Diagnostic endomyocardial biopsy pathology-general biopsy considerations, and its use for myocarditis and cardiomyopathy: a review. Can J Cardiol 18:55-65

19. Veinot JP (2002) Diagnostic endomyocardial biopsy pathology: secondary myocardial diseases and other clinical indications-a review. Can J Cardiol 18:287-296

20. Verduyn SC, Jungschleger JG, Stengl M, Spätjens RLHMG, Beekman JD, Vos MA (2004) Electrophysiological and proarrhythmic parameters in transmural canine left-ventricular needle biopsies. Pflügers Arch 449:115-122

21. Zenati MA, Bonanomi G, Chin AK, Schwartzman D (2003) Left heart pacing lead implantation using subxiphoid videopericardioscopy. J Cardiovasc Electrophysiol 14:949-953 
Milan Stengl, Christian Ramakers, Dirk W. Donker, Ashish Nabar, Andrew V. Rybin, Roel L.H.M.G. Spätiens, Theo van der Nagel, Will K.W.H. Wodzig, Karin R. Sipido, Gudrun Antoons, Antoon F.M. Moorman, Marc A. Vos and Paul G.A. Volders.

Cardiovasc Res. 2006;72:90-100

Circulation. 2004;110:319-320, 1531 Suppl. S. 


\section{Temporal patterns of electrical remodeling in}

canine ventricular hypertrophy

Focus on $I_{K s}$ downregulation and blunted B-adrenergic activation 


\section{ABSTRACT}

Objectives Electrical remodeling in cardiac hypertrophy often involves the downregulation of $\mathrm{K}^{+}$currents, including $B$-adrenergic $(B-A)$ sensitive $I_{\mathrm{Ks}}$. Temporal patterns of ion-channel downregulation are poorly resolved. In dogs with complete atrioventricular block (AVB), we examined (1) the time course of molecular alterations underlying $I_{\mathrm{Ks}}$ downregulation from acute to chronic hypertrophy; and (2) concomitant changing responses of repolarization to $B$-adrenergic receptor (B-AR) stimulation.

Methods and Results Serial left-ventricular (LV) biopsies were collected from anesthetized dogs during sinus rhythm (SR; control) and at 3, 7 and 30 days of AVB. KCNQ1 mRNA and protein decreased within 3 days (protein expression $58 \pm 10 \%$ of control), remaining low thereafter. B1-AR mRNA and protein decreased more gradually to $53 \pm 8 \%$ at 7 days. In chronic-AVB LV myocytes, $\mathrm{I}_{\mathrm{Ks}}$-tail density was reduced: $1.4 \pm 0.3 \mathrm{pA} / \mathrm{pF}$ versus $2.6 \pm 0.4 \mathrm{pA} / \mathrm{pF}$ in controls. B-A enhancement of $\mathrm{I}_{\mathrm{ks}}$ was reduced. Isoproterenol shortened action-potential duration in control cells, while causing heterogeneous repolarization responses in chronic AVB. B-A early afterdepolarizations were induced in 4 of 13 chronic-AVB cells, but not in controls. In intact conscious dogs, isoproterenol shortened QT $_{c}$ at SR (by $-8 \pm 3 \%$ from $\left.295 \mathrm{~ms}\right)$, left it unaltered at 3 days AVB $(+1 \pm 3 \%$ from $325 \mathrm{~ms})$ and prolonged $\mathrm{QT}_{\mathrm{c}}$ at 30 days $(+6 \pm 3 \%$ from $365 \mathrm{~ms})$.

Conclusions Profound decrease of KCNQ1 occurs within days after AVB induction and is followed by a more gradual decrease of B1AR expression. Downregulation and blunted B-A activation of $\mathrm{I}_{\mathrm{Ks}}$ contribute to the loss of $B-A$-induced shortening of ventricular repolarization, favoring proarrhythmia. Provocation testing with isoproterenol identifies repolarization instability based on acquired channelopathy. 


\section{INTRODUCTION}

Electrical remodeling in cardiac hypertrophy often comprises the downregulation of sarcolemmal $\mathrm{K}^{+}$ currents, including the delayed-rectifier $\mathrm{K}^{+}$current $\mathrm{I}_{\mathrm{Ks}}[1,2]$. $\mathrm{K}^{+}$-current downregulation may act to prolong the action-potential duration and enhance excitation-contraction coupling during chronic overload. However, it can confer exaggerated spatial and temporal heterogeneities of ventricular repolarization, rendering the heart more susceptible to deleterious tachyarrhythmia [3]. Temporal patterns of ion-channel downregulation during chronic overload are still poorly resolved.

In the canine model of chronic complete atrioventricular block (AVB) and ventricular hypertrophy, increased spatial $[1,4]$ and temporal dispersion of repolarization [5] predispose to acquired torsades de pointes and sudden cardiac death [6]. At the cellular level, $\mathrm{K}^{+}$currents $\mathrm{I}_{\mathrm{Ks}}$ and $\mathrm{I}_{\mathrm{Kr}}$ are reduced [1], whereas sarcoplasmic reticulum $\mathrm{Ca}^{2+}$ release and $\mathrm{I}_{\mathrm{NaCa}}$ are enhanced, especially at slow heart rates [7]. An increase in subsarcolemmal $\mathrm{Na}^{+}$concentration underlies the altered $\mathrm{Ca}^{2+}$ handling [8]. Previous work has shown that the reduction of $\mathrm{I}_{\mathrm{Ks}}$ in chronic AVB can be due to a down-regulation of KCNQ1-and KCNE1-gene transcription in the basal and midlateral parts of the left-(LV) and right-ventricular wall [9]. In the interventricular septum only KCNO1 mRNA is decreased [10]. These findings indicate regional heterogeneity of ion-channel remodeling in the overloaded myocardium. Until now the time courses of electromolecular changes have remained unclear, especially in the early phase of overload.

Modern insights support the contention that an intact basal $I_{\mathrm{Ks}}$ expression plus B-adrenergic receptor ( $B-A R$ ) stimulation are both essential for $I_{\mathrm{ks}}$ to figure in repolarization, which has been demonstrated in the rabbit $[11]$, dog $[12,13]$ and human heart [14]. This suggests that the separate or combined loss of $I_{K s}$-channel subunits and $I_{K s}$-relevant $B$-adrenergic (B-A) signaling molecules in acquired cardiac pathology carries a risk of repolarization instability and arrhythmia. In relation to this, failure of appropriate action-potential shortening to B-AR stimulation, and torsades-like arrhythmias have previously been reported in LV wedge preparations under pharmacological $I_{\mathrm{Ks}}$ inhibition $[15,16]$.

The present study was designed to examine the time course of molecular alterations underlying $I_{\mathrm{Ks}}$ downregulation in the canine LV after AVB induction. We quantified the mRNA and protein expression of KCNO1 and KCNE1 subunits and B-AR subtypes in serial myocardial biopsies, and measured catecholamine levels in blood plasma. Concomitant changes of cellular and in-vivo repolarization were closely examined. We hypothesized that the propensity of repolarization to shorten upon B-AR stimulation would be undermined after $A V B$, favoring proarrhythmia during maintained overload. 


\section{METHODS}

Animal handling was in accordance with the 'European Directive for the Protection of Vertebrate Animals Used for Experimental and Other Scientific Purposes (86/609/EU)' .The experiments were approved by the Committee for Experiments on Animals of Maastricht University and conformed with the Guide for the Care and Use of Laboratory Animals published by the US National Institutes of Health (NIH Publication No. 85-23, revised 1996). Adult mongrel dogs of either gender were used. Premedication consisted of $10 \mathrm{mg}$ oxycodone $\mathrm{HCl}, 1 \mathrm{mg}$ acepromazine maleate and $0.5 \mathrm{mg}$ atropine sulphate i.m. Complete anesthesia was induced by thiopental $(20 \mathrm{mg} / \mathrm{kg}$ i.v.) and maintained by inhalation of halothane (0.5-1.0\%) and $\mathrm{N}_{2} \mathrm{O}$ and $\mathrm{O}_{2}$ (2:1). Ampicilline (1000 mg) was administered before and after the experiment. For additional analgesia, buprenorphine was given $(0.015 \mathrm{mg} / \mathrm{kg}$ i.m.) after the operation.

\section{In-vivo studies}

AVB was induced by radiofrequency catheter ablation of the His bundle under anesthesia $\left(n_{\text {dogs }}=31\right)$. In-vivo experiments were performed serially at sinus rhythm (SR) and at 0, 3, 8 and 30 days of $A V B$, using each dog as its own control. Venous blood samples were collected from these trained animals at conscious state (after minutes of quiescence) for determination of plasma catecholamines (see below). Premedication was subsequently administered to achieve conscious sedation, and a standard 6-lead ECG was registered along with a unipolar recording at the chest position corresponding to $\mathrm{V} 4$. Under these conditions, a short-lasting B-A challenge with isoproterenol ( $1 \mu \mathrm{g} / \mathrm{kg}$, i.v.; provocation testing) was given to increase heart rate $>25 \%$ during SR or idioventricular rhythm, followed by relaxation $\left(\mathrm{n}_{\text {dogs }}=6\right)$. The QT interval was measured and corrected for heart rate by Van-de-Water's formula: $\mathrm{OT}_{c}=\mathrm{OT}-0.087$ (RR - 1000) [17]. Beat-to beat variability of QT interval was quantified as short-term variability of 30 consecutive beats: $S T V=\Sigma\left(O T_{n}-O T_{n}\right.$ $+1) / 30 \times \sqrt{ } 2[5]$.

In 12 animals, a minimally-invasive procedure was used for biopsy sampling under closed-chest conditions, allowing fast recovery of the dogs and permitting repetitive sampling at SR and 3, 7 and 30 days of AVB. Intramural needle biopsies $(1 \times 1 \times 10 \mathrm{~mm})$ extending from the epicardium into the LV wall were obtained from the apicolateral region under fluoroscopic guidance with a commercially-available automated biopsy device (14-G, Acecut, TSK Laboratory, Japan). This device was percutaneously inserted into the thorax, i.e., through a small dermal incision near the processus xyphoideus. At least 2 biopsies were obtained per experiment. Tissue quality was excellent in nearly all samples ( $>98 \%)$. Histology confirmed minimal injury to the myocardium at the sampling sites. Biopsies were frozen to $-80^{\circ} \mathrm{C}$ in isopentane chilled with liquid nitrogen and then stored until molecular analysis. Dogs were monitored by echocardiography after this procedure to exclude cardiac tamponade, although in our experience this closed-chest approach is generally uncomplicated $(>95 \%)$ and fatal outcome is rare $(<3 \%)$. Such complications did not occur in this study. 
Table $1 \mid$ Primers

\begin{tabular}{|c|c|c|c|c|}
\hline Transcript & Forward $(\mathrm{F}) /$ reverse $(\mathrm{R})$ & $\mathrm{T}_{\text {Anneal }}\left({ }^{\circ} \mathrm{C}\right)$ & Amplicon length (bp) & NCBI Accession number \\
\hline \multirow[t]{2}{*}{ KCNQ1 } & F: GTCGAGTTTGGCAGCTATGCA & 60 & 274 & Not available \\
\hline & R: CGCAAAGAAGGAGATGGCAA & & & \\
\hline \multirow[t]{2}{*}{ KCNE1 } & F: ATGCTGAGTTACATCCGCTC & 57 & 98 & Not available \\
\hline & R: TACGCCTTGTCCTTCTCCT & & & \\
\hline \multirow[t]{2}{*}{$\beta 1$-adrenergic receptor } & F: TACAACGACCCCAAGTGCT & 55 & 100 & U73207 \\
\hline & R: AGGTACACGAAGGCCATGA & & & \\
\hline \multirow[t]{2}{*}{$\beta 2$-adrenergic receptor } & F: АATCCСCTTATCTACTGCCG & 55 & 93 & U73206 \\
\hline & R: ATTCCCATAGGCCTTCAGG & & & \\
\hline \multirow[t]{2}{*}{$\beta 3$-adrenergic receptor } & F: TTCTGTCCCTGACTCCATCA & 55 & 106 & U92468 \\
\hline & R: AAGTCCTCCTGACACTGCTGT & & & \\
\hline
\end{tabular}

\section{Analysis of plasma catecholamines}

Blood samples $(10 \mathrm{~mL})$ were collected into tubes containing lithium heparin as the anticoagulant and transported to the laboratory on ice within $30 \mathrm{~min}$. Samples were centrifuged at $3000 \mathrm{rpm}$ for $10 \mathrm{~min}$ at $4^{\circ} \mathrm{C}$. $100 \mu \mathrm{L}$ of glutathione $(95 \mathrm{~g} / \mathrm{L})$ was added to $2 \mathrm{~mL}$ plasma before storage at $-20^{\circ} \mathrm{C}$. For analysis, plasma was mixed with an internal standard (3,4-dihydroxybenzylamine-hydrobromide) followed by liquid- liquid extraction with $99 \% \mathrm{n}$-heptane and 1-octanol/acetic acid. After evaporation of the organic phase, $100 \mu \mathrm{L}$ of the acetic acid layer was injected and analyzed by reversed phase-high performance liquid chromatography with electrochemical detection.

\section{mRNA and protein expression studies}

Total RNA was isolated from the biopsies using the RNEasy Mini kit (Qiagen/Westburg, Leusden, The Netherlands) according to the manufacturer's protocol. DNAsel-digestion was performed after elution of the total RNA from the column. First-strand cDNA was synthesized using $1 \mu \mathrm{g}$ total RNA in combination with an anchored oligo-dT14VN and reverse 18S rRNA primer [18]. Fluorescencebased kinetic real-time PCR was performed using a LightCycler ${ }^{\text {TM }}$ system (Roche Diagnostics Nederland, Almere, The Netherlands) in combination with the intercalating fluorescent dye SYBR Green I [10]. Amplicons (KCNQ1, KCNE1, and B1-, B2-and B3-ARs) were quantified using the LinRegPCR method [19] and were expressed relative to the constitutively active $18 \mathrm{~S}$ rRNA gene. For primers used see Table 1.

Protein expression was analyzed by Western blotting and densitometric quantification. Proteins were extracted from total homogenates derived from unfixed 5 - $\mu$ m-thick cryosections dissolved in equal amounts of lysis buffer containing $62 \mathrm{mmol} / \mathrm{L}$ Tris- $\mathrm{HCl}, 1.25 \mathrm{mmol} / \mathrm{L}$ EDTA, 2\% NP40, 2.5 
$\mathrm{mmol} / \mathrm{L}$ PMSC, $12.5 \mu \mathrm{g} / \mathrm{mL}$ leupeptin, 12.5\% glycerol, $100 \mu \mathrm{g} / \mathrm{mL}$ aprotinin, 2.3\% SDS for $30 \mathrm{~min}$ on ice and sample buffer containing $62 \mathrm{mmol} / \mathrm{L}$ Tris-HCl, 2.3\% SDS, 10\% glycerol, 5\% B-mercaptoethanol, $0.05 \%$ bromophenol blue for $4 \mathrm{~min}$ at $95^{\circ} \mathrm{C}$. Equal amounts of total protein $(30 \mu \mathrm{g} /$ lane), determined by protein assay, were loaded onto each lane of Tris- $\mathrm{HCl}$ precast polyacrylamide gradient gels (4-20\%) (BioRad Laboratories, Hercules, CA, USA). After separation, proteins were transferred to PVDF membranes (BioRad Laboratories, Hercules, CA, USA). Transfer and loading was controlled by Ponceau $S$ and Coomassie brilliant blue staining. Immunostaining was performed with primary antibodies directed against KCNQ1 (1:50, Santa Cruz Biotechnology, Santa Cruz, CA, USA), KCNE1 (1:100, Alomone Labs, Jerusalem, Israel), B1-AR (1:250, Calbiochem, Merck Biosciences, Darmstadt, Germany), and B2AR (1:500, Chemicon, Temecula, CA, USA). Membranes were blocked for at least $15 \mathrm{~min}$ in 2\% nonfat dry milk (NFDM) in Tween-buffer (TWB), containing 0.13\% Tween 20 in phosphate-buffered saline (PBS). Incubation with the primary antibodies was done for $2 \mathrm{~h}$ in $1 \%$ NFDM or $5 \%$ BSA. After $3 \times 5$ min washing in TWB, blots were incubated for $1 \mathrm{~h}$ using the appropriate peroxidase-conjugated secondary antibodies: swine anti-rabbit (1:5000, Dako Cytomation, Glostrup, Denmark), goat anti-chicken (1:10,000, Jackson Immuno Research, West Grove, PA, USA) and bovine anti-goat (1:250, Santa Cruz Biotechnology, Santa Cruz, CA, USA), washed in TWB ( $3 \times 5 \mathrm{~min})$ and visualized on X-ray films (Fujifilm, Rotterdam, The Netherlands) using ECL or ECLplus (Amersham Biosciences, Amersham, UK). Densitometric quantification was performed with Quantity One ${ }^{\circledR}$ software using a GS 800 scanner (BioRad Laboratories, Hercules, CA, USA).

\section{Cellular experiments}

Hearts were quickly excised from anesthetized SR ( $n_{\text {dogs }}=20$; controls) and AVB ( $\left.n_{\text {dogs }}=22\right)$ dogs. For the cell-isolation method see Ref. [1]. A collagenase-digested part of the apicolateral LV wall was dissected and myocytes were isolated from most of the transmural wall except for a rim $(=1.5 \mathrm{~mm})$ of epicardial and endocardial tissue. Only quiescent rod-shaped cells with clear cross-striations were used for the experiments.

Transmembrane potentials were recorded using high-resistance (30-60 M $\Omega$ ) glass microelectrodes filled with $3 \mathrm{~mol} / \mathrm{L} \mathrm{KCl}$. Whole-cell currents were measured using patch pipettes with resistances of 1.0-3.0 M $\Omega$ when filled with internal solution. Experiments were performed at $37 \pm 1^{\circ} \mathrm{C}$. Cell capacitances were $186 \pm 6 \mathrm{pF}\left(\mathrm{n}_{\text {cells }}=54\right)$ in control and $192 \pm 9 \mathrm{pF}\left(\mathrm{n}_{\text {cells }}=36\right)$ in AVB cells.

The external solution contained (in $\mathrm{mmol} / \mathrm{L}$ ): $\mathrm{NaCl} 145, \mathrm{KCl} 4.0, \mathrm{CaCl}_{2} 1.8, \mathrm{MgCl}_{2} 1.0$, glucose 11, HEPES 10, pH 7.4 with $\mathrm{NaOH}$ at $37^{\circ} \mathrm{C}$. In most patch-clamp experiments $\mathrm{KCl}$ was omitted from the external solution to increase $I_{\mathrm{Ks}}$ amplitude and suppress $\mathrm{I}_{\mathrm{K} 1}[1,20]$. The patch-pipette solution contained (in mmol/L): potassium aspartate $125, \mathrm{KCl} 20, \mathrm{MgCl}_{2} 1.0, \mathrm{MgATP} 5, \mathrm{HEPES} 5$, EGTA 10 , $\mathrm{pH} 7.2$ with $\mathrm{KOH}$. L-type $\mathrm{Ca}^{2+}$ current $\left(\mathrm{I}_{\mathrm{Cal}}\right)$ was blocked with nifedipine $(5 \mu \mathrm{mol} / \mathrm{L})$. Almokalant was dissolved in the superfusate $(2 \mu \mathrm{mol} / \mathrm{L})$ to block $\mathrm{K}_{\mathrm{Kr}}$. Isoproterenol was first dissolved in distilled water containing $30 \mu \mathrm{mol} / \mathrm{L}$ ascorbic acid, kept at $5^{\circ} \mathrm{C}$ and in the dark until use. HMR1556 ((3R,4S)-(+)-N[3hydroxy-2,2-dimethyl-6-(4,4,4-trifluorobutoxy)chroman-4yl]-N-methylmethanesulfonamide, 
Aventis Pharma Deutschland $\mathrm{GmbH}$, Sanofi-Aventis, Frankfurt am Main, Germany) was used to block $I_{\mathrm{Ks}}$. This compound is highly selective for $\mathrm{I}_{\mathrm{Ks}}$ : at a concentration of $500 \mathrm{nmol} / \mathrm{L}$ it blocks the current completely [13], whereas other currents are unaffected [21]. HMR1556 was dissolved in dimethyl sulfoxide as $100 \mathrm{mmol} / \mathrm{L}$ stock solution and diluted in the superfusate to achieve a final concentration immediately before experimental application. The final concentrations of dimethyl sulfoxide (maximally $0.1 \%$ ) in the superfusate had no measurable effects on ion currents and action potentials.

\section{Statistics}

The data are expressed as mean \pm SEM. Data comparisons were made with the Student's t-test for unpaired or paired data groups. Serial changes (mRNA, protein expressions; OT intervals) were tested using linear-regression analysis and repeated-measures ANOVA. Differences were considered statistically significant if $\mathrm{P}<0.05$.

\section{RESULTS}

\section{Temporal patterns of mRNA and protein expression of $\mathrm{I}_{\mathrm{Ks}}$ subunits and B-adrenergic receptors during emerging hypertrophy}

The rate of hypertrophic growth was maximal within 1 week of AVB induction, when echographic LV mass increased by $2.2 \pm 0.4 \mathrm{~g} /$ day [22]. At chronic AVB, heart weights (upon sacrifice) were $>30 \%$ heavier than SR controls (306 $\pm 17 \mathrm{~g}$ versus $233 \pm 11 \mathrm{~g}$, respectively; $\mathrm{P}<0.05)$. When corrected for body weight, this difference remained significant: $10.7 \pm 0.3 \mathrm{~g} / \mathrm{kg}$ versus $8.7 \pm 0.5 \mathrm{~g} / \mathrm{kg}$, respectively $(\mathrm{P}<0.05)$, confirming the presence of hypertrophy.

KCNQ1 mRNA decreased to $58 \pm 13 \%$ of control $(\mathrm{P}<0.05)$ already after 3 days of AVB and further down to $42 \pm 9 \%$ at 30 days. KCNQ1 protein paralleled this decline: $58 \pm 10 \%$ of control after 3 days, $53 \pm 10 \%$ at 30 days $(P<0.05$; Figure 1). Expression levels of KCNO1 at chronic AVB match with previous findings [10]. KCNE1 mRNA and protein did not change significantly after AVB at any of the time points (Figure 1).

B1-AR mRNA decreased more gradually to $73 \pm 5 \%$ of control $(P<0.05)$ at 7 days and $63 \pm 8 \%$ at 30 days. B1-AR protein showed a similar significant decline, as illustrated in Figure 1. B2-AR mRNA and protein remained unaltered (Figure 1). Levels of B3-AR mRNA were too low to be detected and quantified reliably, and were not examined by Western blotting.

For control purposes, we collected serial biopsies (7, 14 and 49 days apart) in 3 dogs with sinus rhythm. Western-blot analysis revealed no significant differences in protein expression of KCNQ1 

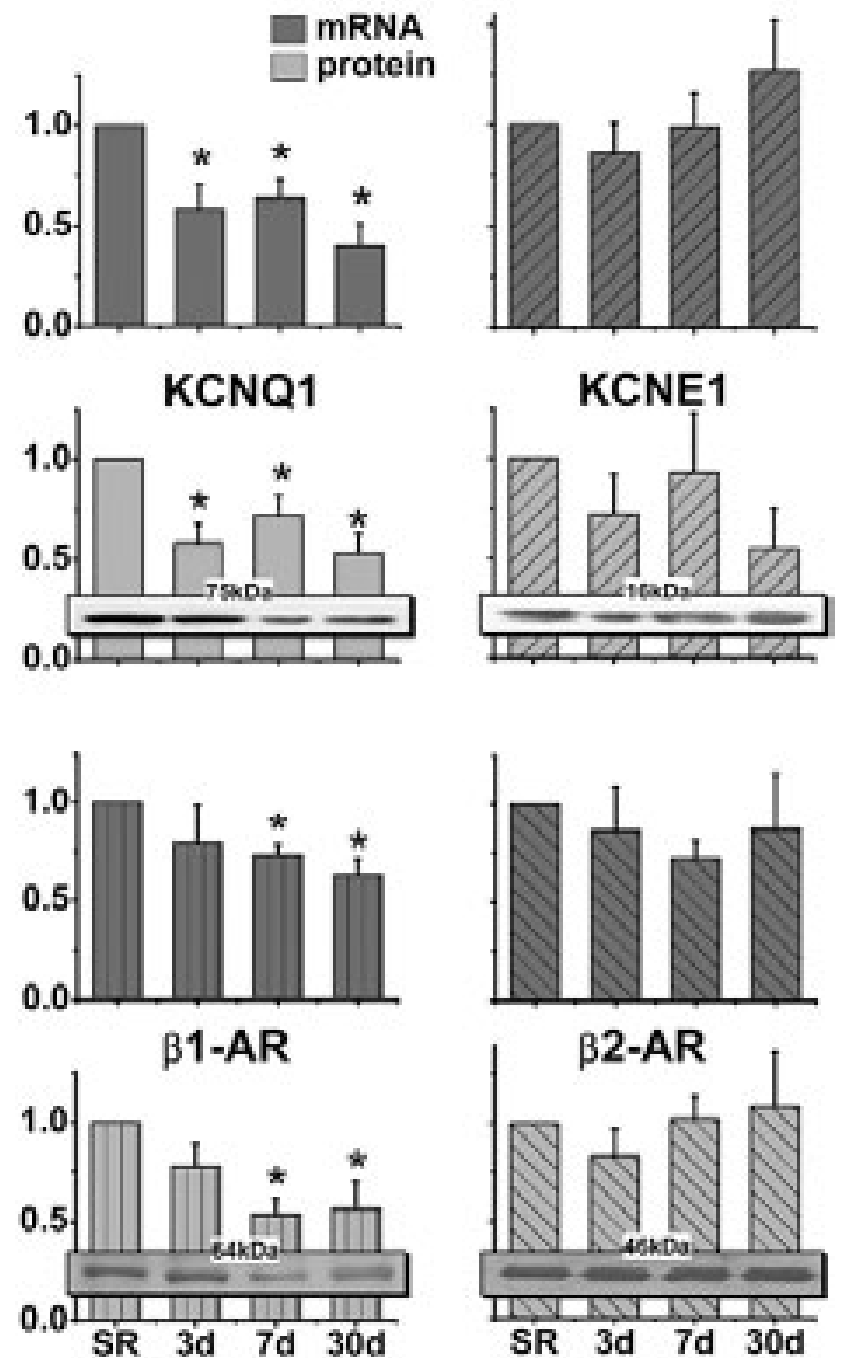

duration of AVB

Figure $1 \mid$ Temporal patterns of mRNA and protein expression of IKs subunits and $\beta$-ARs after AVB. Left upper panels, KCNQ1. Right upper panels, KCNE1. Left lower panels, $\beta 1$-AR. Right lower panels, $\beta 2-A R$. Serial myocardial biopsies were obtained at $S R$ and 3,7 and 30 days of AVB in 12 dogs. ${ }^{*}, \mathrm{P}<0.05$ versus $S R$. 
and B1-AR, indicating that the observed changes after AVB (in the other dogs) were not contaminated by the biopsy sampling itself.

\section{Biophysical properties of downregulated $\mathrm{I}_{\mathrm{Ks}}$}

Biophysical properties of $I_{\mathrm{Ks}}$ in myocytes from dogs with chronic AVB ( $n_{\text {cells }}=15 ; n_{\text {dogs }}=7$ ) were compared to controls $\left(n_{\text {cells }}=19 ; n_{\text {dogs }}=12\right)$. Consistent with protein downregulation, $I_{\mathrm{Ks}}$ density was significantly smaller in chronic AVB: $1.4 \pm 0.3 \mathrm{pA} / \mathrm{pF}$ versus $2.6 \pm 0.4 \mathrm{pA} / \mathrm{pF}$ in controls $(\mathrm{P}<0.05$; tail currents at $-25 \mathrm{mV}$ after $3-\mathrm{s}$ steps to $50 \mathrm{mV}$; Figure $2 \mathrm{~A}$ ). The kinetics of both activation and deactivation of $\mathrm{I}_{\mathrm{Ks}}$ were not different between chronic-AVB and control myocytes (Figure 2B, Table 2). Voltage dependence of $I_{\mathrm{Ks}}$ activation was also similar in chronic-AVB and control cells (Figure 2C). The sensitivity of $I_{\mathrm{Ks}}$ to pharmacological block by HMR1556 remained unchanged in chronic-AVB myocytes $\left(\mathrm{n}_{\text {cells }}=6\right)$ with an IC50 of $81 \mathrm{nmol} / \mathrm{L}$ versus $78 \mathrm{nmol} / \mathrm{L}$ in controls $\left(\mathrm{n}_{\text {cells }}=6\right.$; Figure $\left.2 \mathrm{D}\right)$. $\mathrm{I}_{\mathrm{KS}}$ showed a decreased responsiveness to isoproterenol in chronic-AVB myocytes. SC50 values were $63 \mathrm{nmol} / \mathrm{L}$ for AVB and $30 \mathrm{nmol} / \mathrm{L}$ for control cells. Maximal stimulation at $300 \mathrm{nmol} / \mathrm{L}$ isoproterenol enhanced $I_{K s} 4.0 \pm 0.7$ fold in AVB versus $7.9 \pm 0.7$ fold at control $(P<0.05$; Figure 3$)$. The kinetics of $\mathrm{I}_{\mathrm{Ks}}$ were affected similarly by $B-A R$ stimulation in AVB and control cells (Table 2). For voltage steps from $-50 \mathrm{mV}$ to $20 \mathrm{mV}$, halftimes for activation decreased by approximately $100 \mathrm{~ms}$, whereas halftimes for deactivation increased by about $50 \mathrm{~ms}$.

Activation, depolarizing step from $-50 \mathrm{mV}$ to $+20 \mathrm{mV}$. Deactivation, repolarization to $-25 \mathrm{mV}$. Numbers: $n_{\text {cells }}=10, n_{\text {dogs }}=8$ for chronic $A V B ; n_{\text {cells }}=10, n_{\text {dogs }}=6$ for control. Iso, isoproterenol. ${ }^{*} P<0.05$ versus baseline.

\section{Consequences of $\mathrm{I}_{\mathrm{Ks}}$ downregulation for repolarization response to isoproterenol}

Isoproterenol (100 nmol/L) shortened action-potential duration (APD95) significantly in control cells $\left(n_{\text {cells }}=6 ; n_{\text {dogs }}=3\right.$; Figure $4 A$ ). This was mainly due to the stimulation of $I_{\mathrm{Ks}^{\prime}}$ because block of $\mathrm{I}_{\mathrm{Ks}}$ by HMR1556 $(500 \mathrm{nmol} / \mathrm{L})$ in the presence of isoproterenol reversed it [13]. Heterogeneous responses of repolarization were found in chronic AVB: APD95 remained unchanged in 7, decreased in 4 and increased in 2 myocytes $\left(n_{\text {dogs }}=10 ;\right.$ Figure $4 B$ ). Despite the limited ability of chronic-AVB cells to shorten APD in response to isoproterenol $(100 \mathrm{nmol} / \mathrm{L}) \mathrm{I}_{\mathrm{Ks}}$ still contributed to some degree under these conditions, as evident from the (extra) APD prolongation observed after application of HMR1566 (Figure 4A and B). In the absence of isoproterenol, HMR1556 did not change APD $\left(\mathrm{n}_{\text {cells }}=5\right)$, in line with recent findings in normal dog [13] and human [14].

Early afterdepolarizations (EADs) were induced in 4 of the 13 chronic-AVB cells with isoproterenol (100 nmol/L) alone, and in 6 of 13 myocytes during isoproterenol plus HMR1556 (Figure 5). EADs developed at short (500 ms) and long (1000 and $2000 \mathrm{~ms})$ pacing cycle lengths. They were not observed at baseline. In control myocytes, no EADs were observed under any of these conditions (Figure 5). 
A

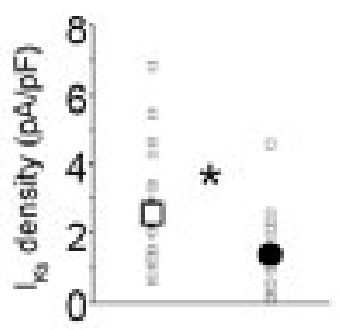

SR CAVB

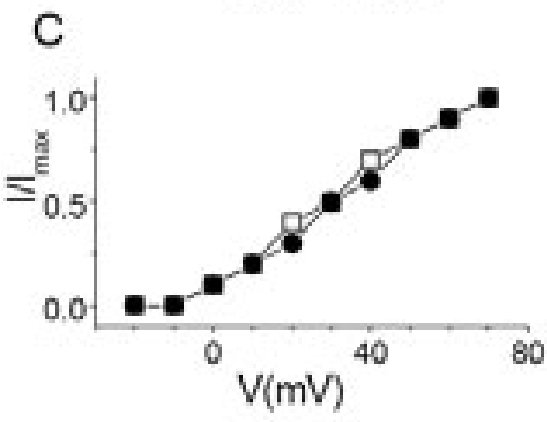

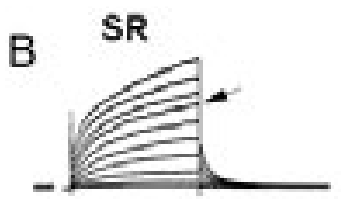

AVB

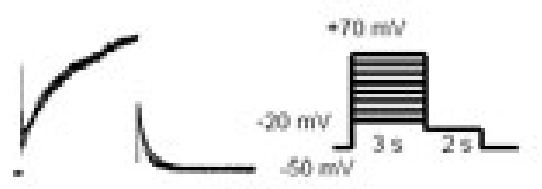

$\mathrm{D}$ 产

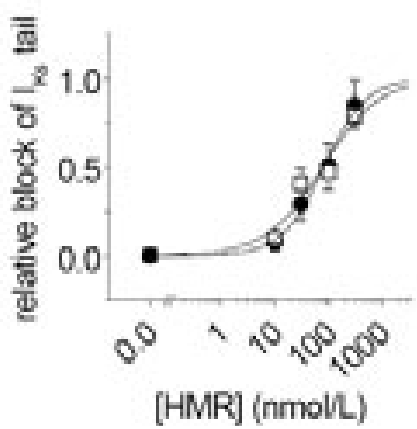

Figure 2 Downregulation of $I_{\mathrm{Ks}}$ in chronic AVB cells. A Chronic AVB (CAVB) led to a decrease of $\mathrm{I}_{\mathrm{Ks}}$ density. B Kinetics of both activation and deactivation of $\mathrm{I}_{\mathrm{Ks}}$ were not different between AVB and control myocytes. Upper panels, arrows indicate $\mathrm{I}_{\mathrm{Ks}}$ traces at depolarizations to $40 \mathrm{mV}$ (SR versus CAVB), which were used for comparison of kinetics after artificial equalization of amplitude (in left panel below; see also Table 2). Vertical bar, 500 pA. Horizontal bar, $1 \mathrm{~s}$. Inset, voltage-clamp protocol. C Voltage dependence of $\mathrm{I}_{\mathrm{Ks}}$ activation was similar in CAVB (filled circles) and control cells (open squares). D Sensitivity of $\mathrm{I}_{\mathrm{Ks}}$ to pharmacological block by HMR1556 remained unchanged in CAVB cells (filled circles). *, $\mathrm{P}<0.05$.

Table $2 \mid$ Kinetics of $\mathrm{I}_{\mathrm{Ks}}$ activation and deactivation

\begin{tabular}{lllll}
\hline & \multicolumn{2}{l}{ Control, halftimes $(\mathrm{ms})$} & \multicolumn{2}{l}{ Chronic AVB, halftimes $(\mathrm{ms})$} \\
\hline \multirow{3}{*}{ Baseline } & Activation & Deactivation & Activation & Deactivation \\
\cline { 2 - 5 } Iso $(100 \mathrm{nmol} / \mathrm{L})$ & $511 \pm 63$ & $128 \pm 13$ & $509 \pm 36$ & $121 \pm 9$ \\
\hline
\end{tabular}

Activation, depolarizing step from $-50 \mathrm{mV}$ to $+20 \mathrm{mV}$. Deactivation, repolarization to $-25 \mathrm{mV}$. Numbers: $\mathrm{n}_{\text {cells }}=10, \mathrm{n}_{\text {dogs }}=8$ for chronic AVB; $n_{\text {cells }}=10, n_{\text {dogs }}=6$ for control. Iso, isoproterenol. ${ }^{*} P<0.05$ versus baseline. 


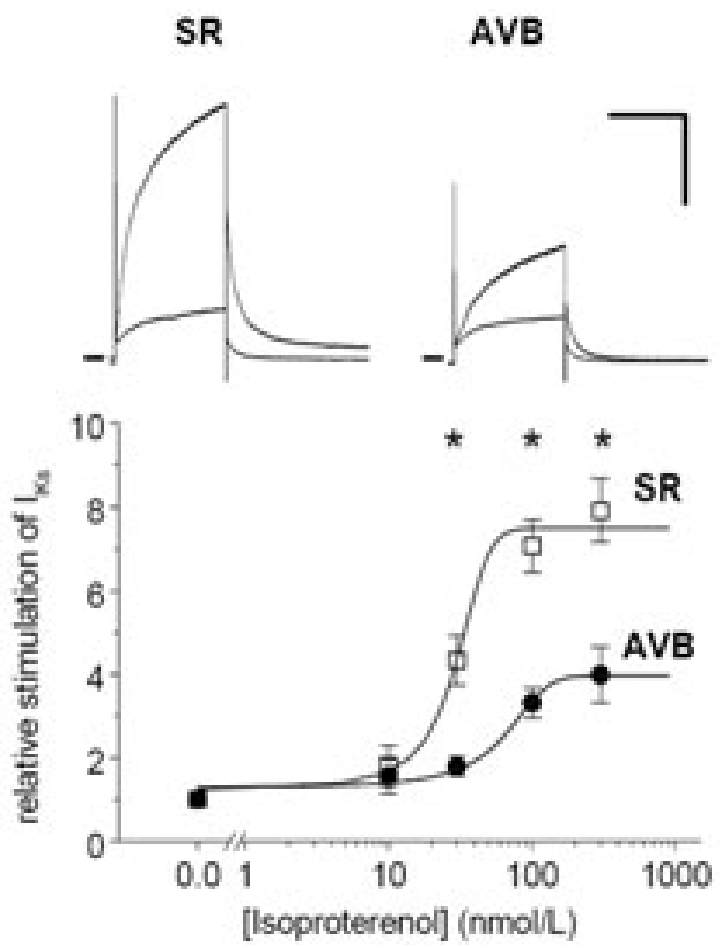

Figure $3 \mid$ Decreased responsiveness of $I_{\text {Ks }}$ to $B-A$ stimulation in chronic-AVB (CAVB) cells. Upper panel, representative examples of $\mathrm{I}_{\mathrm{Ks}}$ at baseline and when increased by B-A stimulation (isoproterenol, 100 $\mathrm{nmol} / \mathrm{L}$ ) in a control (SR) and a CAVB cell. Relative data shown are based on $\mathrm{K}_{\mathrm{Ks}}$-tail amplitudes. Vertical bar, 5 pA/pF. Horizontal bar, 2 s. Lower panel, dose dependence of $I_{\mathrm{Ks}}$ stimulation by isoproterenol at control and CAVB. Inset, voltage-clamp protocol. ${ }^{\star}, \mathrm{P}<0.05$.

A cAMP-activated $\mathrm{Cl}^{-}$current $\left(\mathrm{I}_{\mathrm{C}}\right)$ could also contribute to the action-potential shortening in response to $B-A$ stimulation. In dog ventricle however, such a current is reportedly absent [23], whereas it was also shown that anthracene increased the incidence of EADs by isoproterenol suggesting the presence of cAMP-activated $\mathrm{I}_{\mathrm{Cl}}$ [24]. We performed additional experiments and measured currents during wash-in of $3 \mu \mathrm{mol} / \mathrm{L}$ isoproterenol in conditions that should reveal this $I_{C I}$ $\left(\mathrm{K}^{+}\right.$-and $\mathrm{Na}^{+}$-free solutions with symmetrical $\left[\mathrm{Cl}^{-}\right]$; current measured at $-80 \mathrm{mV}$ ). Current density at baseline was $-0.32 \pm 0.06 \mathrm{pA} / \mathrm{pF}$ in control $\left(\mathrm{n}_{\text {cells }}=7\right)$ and $-0.30 \pm 0.05 \mathrm{pA} / \mathrm{pF}$ in chronic-AVB myocytes $\left(n_{\text {cells }}=7, P=N S\right)$. In the presence of isoproterenol $(3 \mu \mathrm{mol} / \mathrm{L})$ the current was $-0.36 \pm 0.06 \mathrm{pA} / \mathrm{pF}$ and $-0.35 \pm 0.04 \mathrm{pA} / \mathrm{pF}$, respectively $(\mathrm{P}=\mathrm{NS})$. In 4 cells, this current was not sensitive to the $\mathrm{Cl}^{-}$channel blocker niflumic acid $(100 \mu \mathrm{mol} / \mathrm{L})$. These data suggest that if present, the cAMP-dependent $\mathrm{I}_{\mathrm{Cl}}$ is very small and does not contribute to differences in repolarization between control and chronic AVB. 


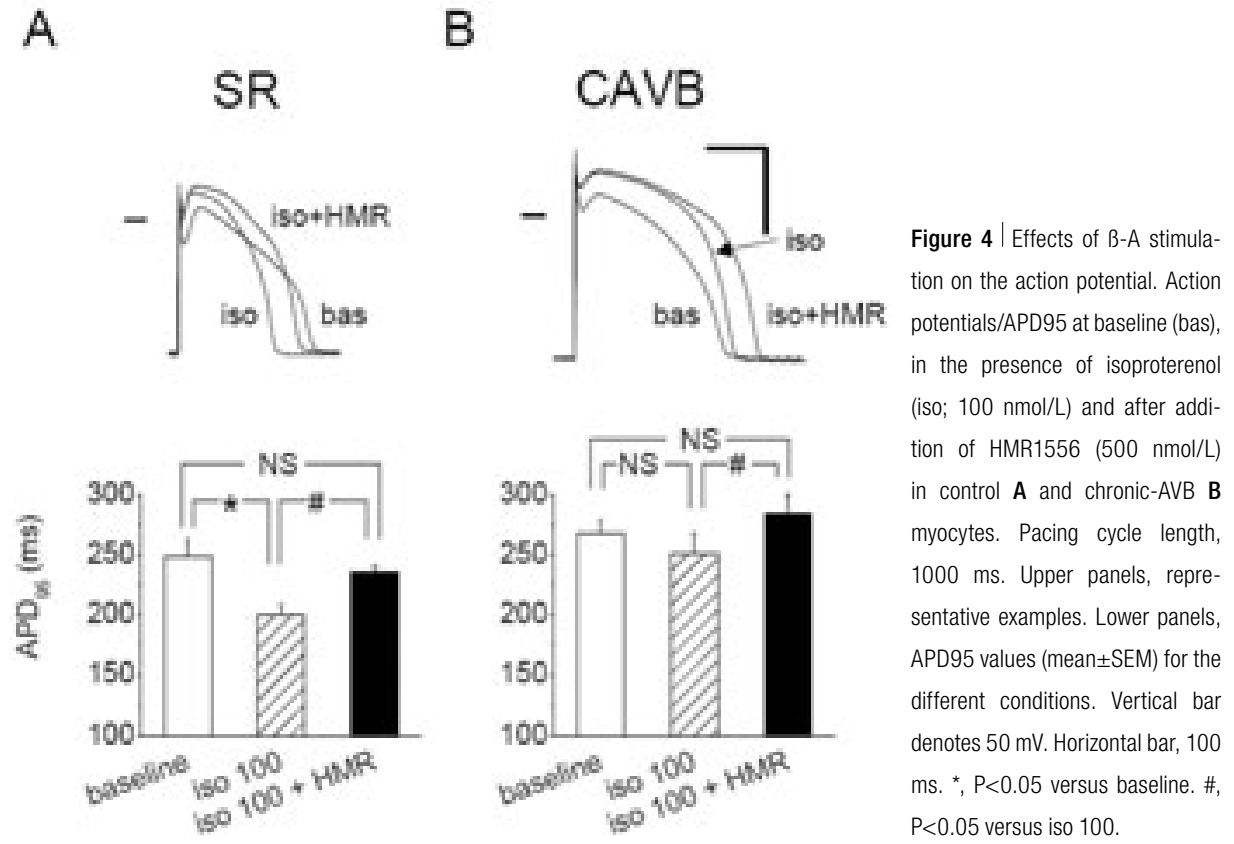

\section{QT responses to isoproterenol in conscious AVB dogs}

To investigate whether repolarization responses to B-AR stimulation were also altered in vivo, we subjected 6 dogs to short-lasting challenges with isoproterenol $(1 \mu \mathrm{g} / \mathrm{kg}$ i.v.; $>25 \%$ increase of heart rate) at SR control and during idioventricular rhythm at 3, 8 and 30 days of AVB.

The heart rate dropped from $128 \pm 12 \mathrm{bpm}$ at $S R$ to $41 \pm 4$ bpm at 3 days $A V B$, remaining low thereafter (Figure 6). At baseline during conscious sedation, QT time (chest lead V4) measured $253 \pm 10$ $\mathrm{ms}$ at SR. It increased thereafter from $371 \pm 12 \mathrm{~ms}$ at 3 days to $414 \pm 7 \mathrm{~ms}$ at 30 days AVB $(P<0.05$ versus 3 days), indicating electrical remodeling [1].

Next we investigated the actions of isoproterenol $(1 \mu \mathrm{g} / \mathrm{kg}$ i.v.). Both in SR and idioventricular rhythm a strong chronotropic effect was noted, as expected: heart rates increased from $128 \pm 12$ bpm to $201 \pm 9 \mathrm{bpm}(\mathrm{SR})$ and from $41 \pm 4 \mathrm{bpm}$ to $58 \pm 5 \mathrm{bpm}$ (3 days AVB), respectively. These chronotropic responses remained proportionally similar at the later stages of AVB (Figure 6B). QT-interval measurements were performed at 2 time points during each experiment: (1) when heart rate increased $25 \%$ above the baseline value (for comparability between experiments; hatched bars Figure $6 \mathrm{~B}$ and $\mathrm{C}$ ); and (2) during peak chronotropic response (black bars Figure 6B and C). 

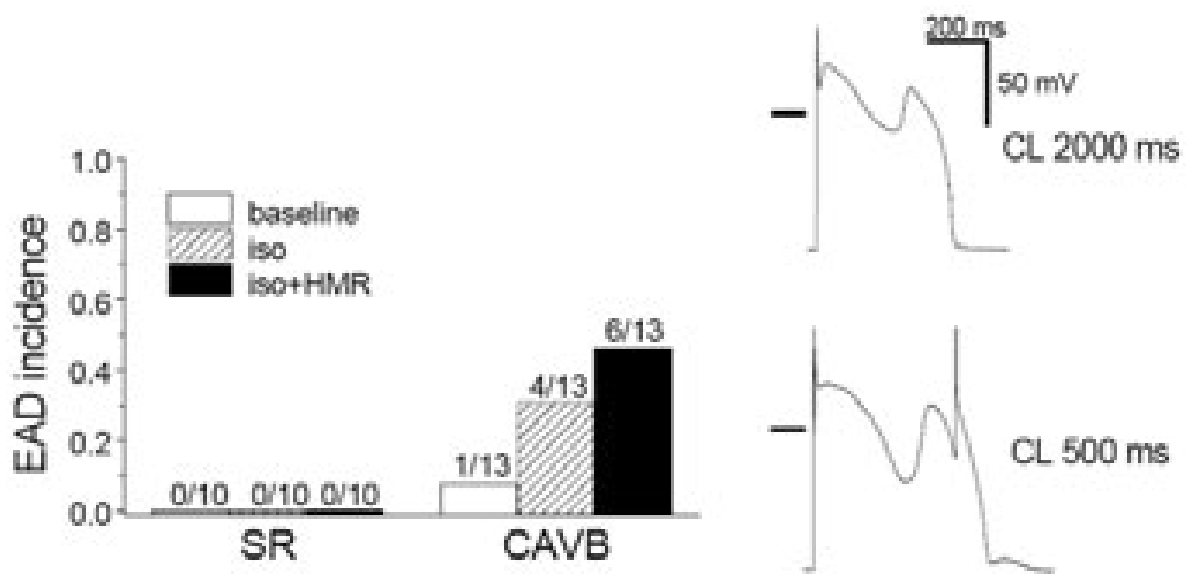

Figure $5 \mid$ EADs in the presence of B-A stimulation in chronic-AVB (CAVB) cells. Incidence of EADs at baseline, in the presence of isoproterenol (iso; $100 \mathrm{nmol} / \mathrm{L}$ ) and after addition of HMR1556 (500 nmol/L) in control (SR) and CAVB cells. Right panels, representative action potentials with EADs in the presence of isoproterenol at pacing cycle lengths (CL) of $2000 \mathrm{~ms}$ and $500 \mathrm{~ms}$.

A profound shortening of repolarization accompanied the peak heart-rate increase at SR: OT decreased from $253 \pm 10$ to $211 \pm 5 \mathrm{~ms}(\mathrm{OT}-17 \% ; \mathrm{P}<0.05)$. At 3 days $A V B, 0 T$ shortened from $371 \pm 12$ ms to $347 \pm 6$ ms (heart rate $+25 \%$; $\mathrm{QT}-6 \%$; $\mathrm{P}<0.05$ ) to $324 \pm 4$ ms (peak response; $\mathrm{QT}-13 \% ; \mathrm{P}<0.05$ ). At 30 days AVB, however, $\mathrm{QT}$ changes during isoproterenol became much less pronounced despite similar increases in heart rate: OT altered from $414 \pm 7$ ms to $409 \pm 11$ ms (heart rate $+25 \%$; OT $-1 \%$; $P=N S$ ) to $392 \pm 10$ ms (peak response; OT $-5 \% ; P<0.05$ ). Individual $0 T$ times were often longer than their baseline values and they varied considerably on a beat-to-beat basis, indicating in-vivo repolarization instability (Figure 6A and D). The latter was not observed at SR or acute AVB. When corrected for heart rate, $\mathrm{QT}_{c}$ was shortened by isoproterenol during $S R$, unchanged at 3 and 8 days $A V B$, and overtly prolonged in chronic AVB (Figure 6C). Beat-to-beat variability of QT (quantified as STV) decreased from $2.5 \pm 0.4 \mathrm{~ms}$ to $1.6 \pm 0.3 \mathrm{~ms}(\mathrm{P}<0.05)$ in response to isoproterenol during $\mathrm{SR}$. At 3 days AVB OT STV remained unchanged ( $4.0 \pm 0.4 \mathrm{~ms}$ to $4.8 \pm 0.7 \mathrm{~ms} ; \mathrm{P}=\mathrm{ns})$, whereas at 30 days it actually increased from $5.4 \pm 0.6 \mathrm{~ms}$ to $7.4 \pm 0.7 \mathrm{~ms}(P<0.05)$ during $B-A$ stimulation (Figure $6 \mathrm{D})$. Ventricular tachycardia was not observed, supposedly because local myocardial B-A stimulation was not intense enough.

\section{Plasma levels of catecholamines}

To assess the influence of natural catecholamines on repolarization, plasma levels of dopamine, epinephrine and norepinephrine were measured in venous blood samples at all time points, including 
A
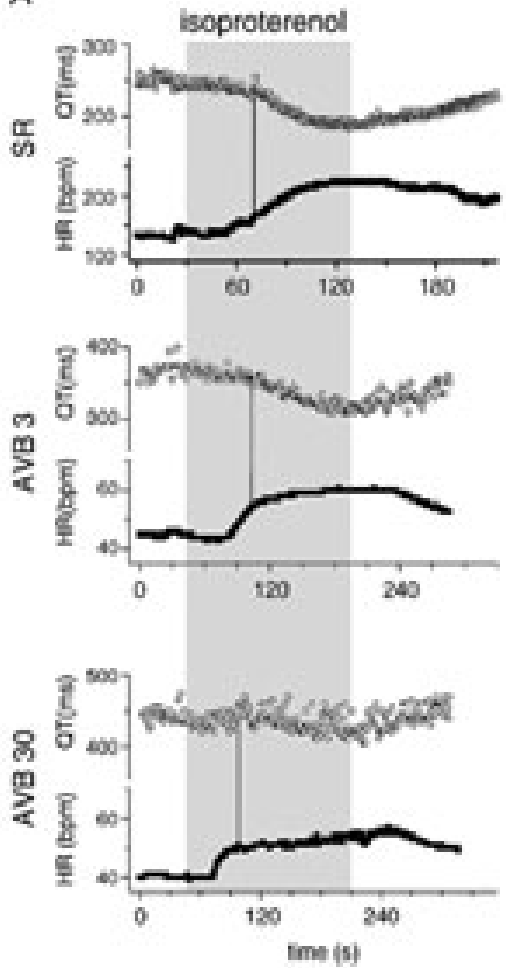

B
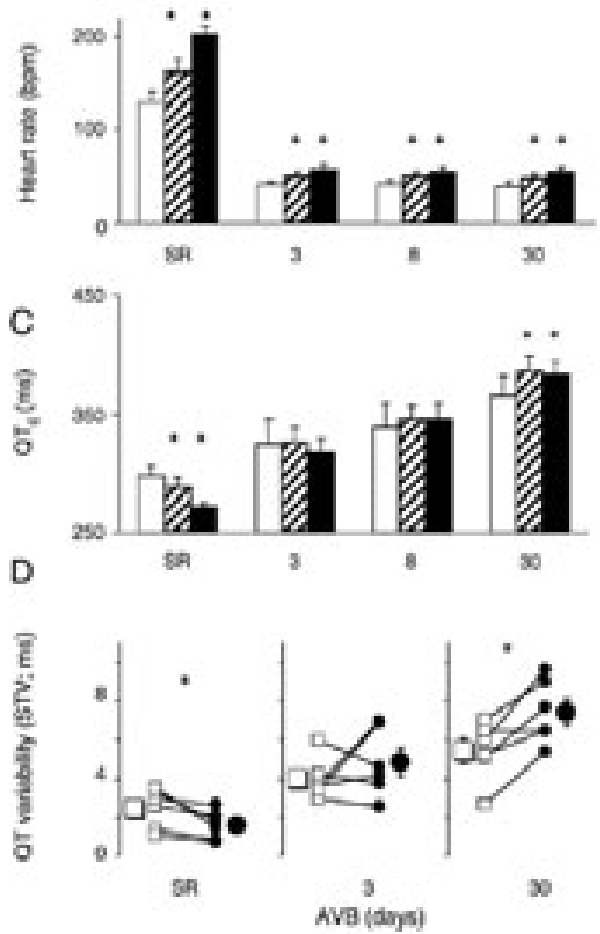

Figure 6 | QT responses to isoproterenol in conscious AVB dogs. A Heart rates (HR; filled squares) and corresponding QT times (open circles) during infusion of isoproterenol ( $1 \mu \mathrm{g} / \mathrm{kg}$, i.v.) in a representative dog during SR (upper panel), and at 3 days (middle panel) and 30 days of AVB (lower panel). The grey zone indicates the period of isoproterenol infusion. Vertical lines indicate the time points of $25 \%$ heart-rate increase (inserted for comparability). B Heart-rate responses to isoproterenol. White bars indicate baseline conditions during SR and after 3, 8, 30 days of AVB. Hatched bars, 25\% heart-rate increase above baseline. Black bars, peak responses. C QT responses to isoproterenol at baseline (white bars) and during isoproterenol infusion (hatched bars, at $25 \%$ heart-rate increase above baseline; black bars, during peak responses of heart rate). D Beat-to-beat variability of QT (quantified as short-term variability; STV) at baseline (open squares) and during isoproterenol (filled circles) in individual dogs (connected by lines) during SR and after 3 and 30 days of AVB. Same symbols at the side indicate mean \pm SEM. *, $\mathrm{P}<0.05$ for isoproterenol versus baseline. 


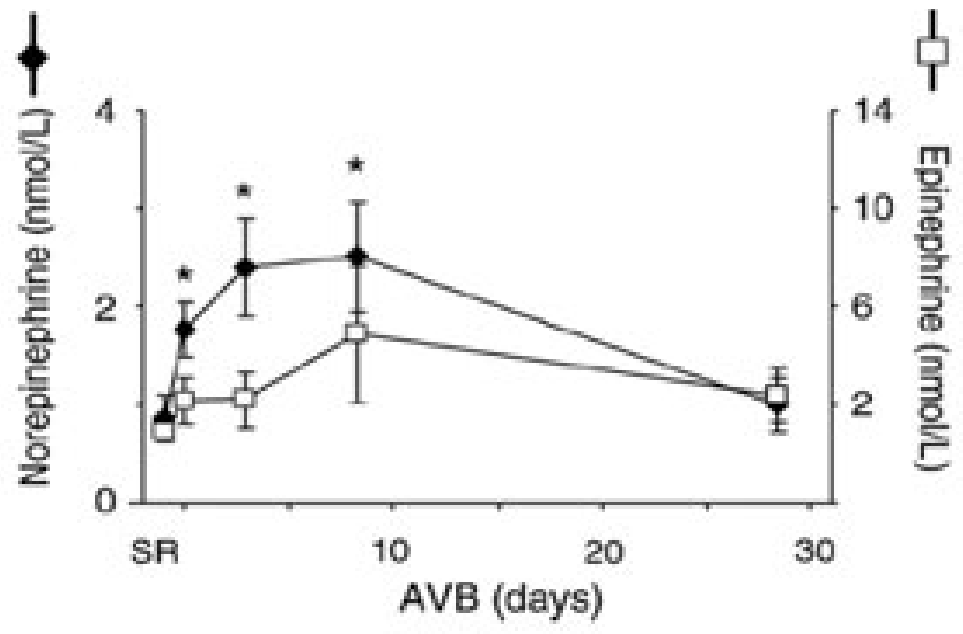

Figure 7 Plasma levels of natural catecholamines during AVB. Concentrations of epinephrine and norepinephrine in venous blood plasma at SR and $0,3,8$ and 30 days of AVB. ${ }^{*}, P<0.05$ versus $S R$.

the recovery phase after induction of AVB ( 0 days). Measured levels of dopamine ranged from 'not detectable' to $0.32 \mathrm{nmol} / \mathrm{L}$ under control conditions (median

$0.12 \mathrm{nmol} / \mathrm{L}$ ) and did not alter during AVB. Likewise, epinephrine concentrations did not change significantly (Figure 7; open squares). Norepinephrine, however, (control concentration $0.87 \pm 0.23$ $\mathrm{nmol} / \mathrm{L}$ ) increased significantly upon induction of AVB ( 0 days) and peaked at $2.50 \pm 0.56 \mathrm{nmol} / \mathrm{L}$ around 8 days AVB. In the chronic phase of AVB thereafter ( $>8$ days), norepinephrine returned to control levels (Figure 7 ; filled diamonds). We speculate that the early rise of this catecholamine is a response mechanism to overcome incomplete circulatory compensation in the first 2 weeks of AVB [22].

\section{DISCUSSION}

The present study demonstrates that a profound decrease of KCNO1 mRNA and protein occurs within days after induction of AVB in the apicolateral region of the canine LV. It is followed by a more gradual decrease of B1-ARs. The kinetics of downregulated $I_{\mathrm{Ks}}$ and its responsiveness to the selective blocker HMR1556 remain unaltered in AVB myocytes. However, B-AR stimulation of $I_{K s}$ is decreased. At the level of the cellular action potential these molecular and biophysical characteristics translate to the loss of $\mathrm{B}$-A-induced shortening of repolarization, favoring the occurrence 
of EADs. In conscious dogs, $\mathrm{QT}$ and $\mathrm{QT}_{\mathrm{c}}$ responses to $\mathrm{B}-\mathrm{A}$ challenges confirm the $\mathrm{I}_{\mathrm{Ks}}$-related loss of ventricular repolarization shortening in chronic $A V B$, when beat-to-beat $Q T$ instability is also eminent.

\section{Downregulation of $\mathrm{I}_{\mathrm{Ks}}$ and $B$-adrenergic regulation}

Downregulation of $I_{\mathrm{KS}}$ at the chronic stage of AVB has been reported previously in the dog $[1,9]$ and rabbit [25]. Decreases in $I_{\mathrm{Ks}}$ were also found in other animal models of cardiac hypertrophy and/or failure $[2,26,27]$, and in humans [28]. In the present study we closely analyzed the biophysical properties of downregulated $I_{\mathrm{Ks}}$ in chronic-AVB myocytes. The unaltered kinetics and responsiveness to HMR1556 indicated that reductions in channel numbers, rather than conformational changes, underlie the diminution of this current. Another aspect of $\mathrm{I}_{\mathrm{Ks}}$ downregulation was the finding, that, in contrast to the reduction of KCNQ1, KCNE1 did not change. We observed a similar differential response in the canine interventricular septum after chronic AVB [10]. However, reductions of both KCNQ1 and KCNE1 mRNA and protein were found in the basal and midlateral parts of the LV and right ventricle [9]. In the latter study, mRNA expression was quantified using a combination of Northern blot and multiplex competitive real-time PCR, whereas in the recent septum study [10] and the present one fluorescence-based kinetic real-time PCR was employed. The latter is a more accurate method. To exclude a methodological reason for the apparent discrepancies in KCNE1 results, we redetermined KCNE1 mRNA in tissue from the basal and midlateral LV of normal and chronic-AVB dogs (as in Ref. [9]) using the fluorescence-based kinetic real-time PCR (as in the present study), and found a similar decrease of expression as described previously [9]. Based on these data, we consider regional heterogeneity of $I_{\mathrm{Ks}}$-subunit remodeling in the LV, even among $\alpha$-and $\beta$-channel subunits, the most likely explanation for the different results in the three studies.

The question arises why the decrease of KCNO1 did not change the kinetics of $\mathrm{I}_{\mathrm{Ks}}$. Wang et al. [29] examined the influence of various ratios of KCNQ1 : KCNE1 on the current kinetics of the assembled $I_{\mathrm{Ks}}$ channel. Ratios of 2:1 and 1:1 resulted in similar current characteristics, and both closely resembled the activation kinetics and voltage dependence of native $I_{\mathrm{Ks}}$ [29]. Thus, a unilateral decrease of KCNQ1 of $~ 50 \%$ (as in AVB) would not be expected to affect the kinetics of native $\mathrm{I}_{\mathrm{Ks}}$ significantly, in line with the present findings.

Stimulation of $I_{\mathrm{Ks}}$ by B1-ARs depends predominantly (if not exclusively) on protein-kinase-A stimulation of KCNQ1 subunits [30]. Modulation via B2-ARs has also been suggested, mainly by the finding that cardiac $I_{\mathrm{Ks}}$ density is increased in transgenic mice expressing fusion proteins of $I_{\mathrm{Ks}}$ subunits and human B2-ARs [31]. B2-A signaling could modulate the function of these $I_{\mathrm{Ks}}$ channels even in the absence of exogenous B-A agonists [31]. B3-A regulation of $I_{\mathrm{Ks}}$ has also been reported, although with different results in different models: B3-AR stimulation enhanced $I_{\mathrm{Ks}}$ in Xenopus oocytes expressing human KvLOT1/MinK channels [32], but inhibited the current in guinea-pig ventricular myocytes [33]. From the three receptor subtypes ( $B 1, B 2$ and $B 3)$ examined in the present study, only $B 1$ expression showed a clear and sustained decrease compatible with the decreased $B-A$ 
responsiveness of $I_{\mathrm{Ks}}$ in AVB cells. We cannot exclude that, apart from the decreases of KCNQ1 and $\mathrm{B1}-\mathrm{AR}$, other elements of the $I_{K s}$-macromolecular signaling complex [34] or B-A cascade are also involved in the downregulation of $\mathrm{I}_{\mathrm{Ks}}$.

The effect of B-AR downregulation is probably not the same for all ion channels. We found that the maximal response of $\mathrm{I}_{\mathrm{CaL}}$ during depolarizing steps to $0 \mathrm{mV}$ was the same in control and chronic AVB (manuscript under revision). Differences in B-A responsiveness between $I_{C a L}$ and $I_{K s}$ could be explained by different coupling of these channels to their receptors and/or signaling cascades. Isoproterenol stimulation of L-type $\mathrm{Ca}^{2+}$ channels results mainly from a local activation of the signaling cascade [35]. At the present time there is no such data available for $\mathrm{I}_{\mathrm{Ks}}$ channels.

\section{Temporal patterns of remodeling at the molecular and in-vivo level: do they match?}

Using a minimally-invasive procedure under closed-chest conditions, we obtained LV myocardial biopsies in a serial manner. Thus, follow-up of the transcriptional expression of $\mathrm{I}_{\mathrm{Ks}}$-channel subunits and other $I_{K s}$-relevant proteins (including B-AR subtypes) was possible using each dog as its own control, thereby minimizing problems of biological variability. Such problems are often encountered in group comparisons when different animals are used. To the best of our knowledge, this is the first study to describe serial molecular-expression patterns in LV myocardial tissue from individual dogs. We believe that this approach holds great promise for future studies of myocardial remodeling in chronic pathological conditions.

When we align the patterns of KCNQ1 and B1-A downregulation with the altering QT responses in vivo and the changes of plasma norepinephrine, an interesting picture emerges. Considering that $\mathrm{I}_{\mathrm{Ks}}$ downregulation is a major determinant of the AVB-induced repolarization prolongation [1], the fast decrease of KCNQ1 (at 3 days; Figure 1) apparently contrasts with the much slower response of OT and $\mathrm{OT}_{\mathrm{c}}$ prolongation by remodeling (after $>8$ days of AVB; Figure 6 ). However, the contribution of $I_{\mathrm{Ks}}$ to repolarization depends critically on B-AR stimulation [13] and therefore this system must be taken into account when explaining the temporal differences. We find it likely that the early rise of norepinephrine (Figure 7) 'neutralizes' the loss of KCNQ1, effectively maintaining a pseudonormal $\mathrm{I}_{\mathrm{Ks}}$ in the first week of AVB. In the period thereafter, the waning of norepinephrine levels and the additional downregulation of B1-ARs (Figure 1) gradually uncovers the QT prolongation. In a similar time-dependent manner, the loss of B-A-induced shortening of ventricular repolarization could be explained.

The lack of an effect of isoproterenol on the $\mathrm{QT}_{\mathrm{c}}$ interval at 3 and 7 days AVB (Figure $6 \mathrm{C}$ ) could arise from the influence of high levels of endogenous norepinephrine in the ventricles at this stage. This could have obscured the effect of the exogenous B-A agonist.

Different mechanisms control the chronotropic responses to isoproterenol during $S R$ and $A V B$, i.e., via stimulation of the sinus node and via stimulation of distal His-bundle cells, Purkinje cells or even ventricular working myocardium, respectively. The sensitivity of these pacemaking tissues to isoproterenol may not be the same in the two conditions. 


\section{Consequences of $\mathrm{I}_{\mathrm{Ks}}$ downregulation}

In control myocytes, B-A activation shortened the APD consistently, which indicated that the stimulation of outward currents (mainly $I_{\mathrm{Ks}}$ ) prevailed over the stimulation of inward currents (mainly $I_{\text {CaL }}$ ). In chronic-AVB cells, heterogeneous APD responses were observed, likely as the result of $I_{\mathrm{Ks}}$ downregulation in the presence of an unchanged $I_{\mathrm{CaL}}$ and enhanced $I_{\mathrm{NaCaX}}$ [7]. The reduced $I_{\mathrm{Ks}}$ still contributed to repolarization during B-AR stimulation, as evident from the (extra) APD prolongation after administration of HMR1566 (Figure 4). Under these conditions (isoproterenol plus HMR1566) the frequent generation of EADs in chronic-AVB cells, and their absence in control myocytes (Figure 5), strongly suggested that other ionic changes (e.g., enhanced $\mathrm{Na}^{+}-\mathrm{Ca}^{2+}$ exchange [7]) also contributed to the proarrhythmia. Taken together our results indicate that the already labile ventricular repolarization in chronic AVB is adversely challenged during episodes of intense $B-A$ activation. The generation of $B-A$ EADs would exaggerate this repolarization lability and also trigger abnormal impulses.

Approximately $10 \%$ of the dogs with chronic AVB die suddenly. In some of these, sudden death occurred during excitement, as witnessed by animal technicians. In other animals, ambulatory electrocardiographic recordings revealed polymorphic ventricular tachycardia as the mode of death [6]. Tachycardia was immediately preceded by an accelerated idioventricular rhythm and short-coupled ventricular beats from different foci [6]. The data suggest a significant contribution of the sympathetic nervous system to the initiation of these arrhythmic events. Given the findings of KCNO1 downregulation and B-A proarrhythmia in this dog model, there is an interesting similarity with the human congenital long-OT 1 syndrome. Sympathetic triggers of torsades de pointes, as during exercise and emotion, have long been recognized in long-0T-1 patients [36]. In latent carriers of KCNO1 mutations, intravenous epinephrine can unmask the abnormal repolarization [37], much like our findings with isoproterenol in dogs with AVB. To our knowledge, the present study is the first to describe provocation testing using isoproterenol in acquired cardiac channelopathy.

\section{Acknowledgments}

This study was supported by The Netherlands Organization for Health and Development, The Hague (ZonMw 90602-068 to P.G.A.V.), The Netherlands Heart Foundation, The Hague (NHS 98.042 to C.R.), the 'Stichting Hartsvrienden Rescar', Maastricht, The Netherlands (to P.G.A.V.), and the United States National Heart, Lung, and Blood Institute (USPHS-NHLBI grant HL-67101 to A.V.R.). Monique M.J. de Jong, Departments of Cardiology and CardioThoracic Surgery, Maastricht, and Jet D.M. Beekman, Department of Medical Physiology, University Medical Center Utrecht, The Netherlands provided excellent technical assistance. Michel E.F.H. Pereboom, Department of Clinical Chemistry, Academic Hospital Maastricht, performed the catecholamine analyses. The primers for canine KCNQ1 and KCNE1 genes were kindly provided by Robert Dumaine, PhD, Department of Physiology and Biophysics, Faculty of Medicine and Health Sciences, University of Sherbrooke, OC, Canada. 
CHAPTER 5 | TEMPORAL PATTERNS OF ELECTRICAL REMODELING IN VENTRICULAR HYPERTROPHY

The authors are grateful to Jos G. Maessen, MD, PhD, Department of CardioThoracic Surgery, Cardiovascular Research Institute Maastricht and Academic Hospital Maastricht for his role in establishing the serial-biopsy technique. 


\section{REFERENCES}

1. Volders PGA, Sipido KR, Vos MA, Spätjens RLHMG, Leunissen JDM, Canmeliet E, et al. Downregulation of delayed rectifier $\mathrm{K}^{+}$currents in dogs with chronic complete atrioventricular block and acquired torsades de pointes. Circulation 1999;100:2455-61.

2. Tsuji Y, Opthof T, Kamiya K, Yasui K, Liu W, Lu Z, et al. Pacing-induced heart failure causes a reduction of delayed rectifier potassium currents along with decreases in calcium and transient outward currents in rabbit ventricle. Cardiovasc Res 2000;48:300-9.

3. Tomaselli GF, Zipes DP. What causes sudden death in heart failure? Circ Res 2004;95:754-63.

4. Kozhevnikov D0, Yamamoto K, Robotis D, Restivo M, El-Sherif N. Electrophysiological mechanism of enhanced susceptibility of hypertrophied heart to acquired torsade de pointes arrhythmias: tridimensional mapping of activation and recovery patterns. Circulation 2002;105:1128-34.

5. Thomsen MB, Verduyn SC, Stengl M, Beekman JDM, de Pater G, van Opstal J, et al. Increased shortterm variability of repolarization predicts $d$-sotalol-induced torsades de pointes in dogs. Circulation 2004;110:2453-9.

6. van Opstal JM, Verduyn SC, Leunissen HDM, de Groot SHM, Wellens HJJ, Vos MA. Electrophysiological parameters indicative of sudden cardiac death in the dog with chronic complete AV-block. Cardiovasc Res 2001;50:354-61.

7. Sipido KR, Volders PGA, de Groot SHM, Verdonck F, Van de Werf F, Wellens HJJ, et al. Enhanced Ca ${ }^{2+}$ release and $\mathrm{Na} / \mathrm{Ca}$ exchange activity in hypertrophied canine ventricular myocytes: potential link between contractile adaptation and arrhythmogenesis. Circulation 2000;102:2137-44.

8. Verdonck F, Volders PGA, Vos MA, Sipido KR. Increased $\mathrm{Na}^{+}$concentration and altered $\mathrm{Na} / \mathrm{K}$ pump activity in hypertrophied canine ventricular cells. Cardiovasc Res 2003;57:1035-43.

9. Ramakers C, Vos MA, Doevendans PA, Schoenmakers M, Wu YS, Scicchitano S, et al. Coordinated downregulation of KCNQ1 and KCNE1 expression contributes to reduction of $\mathrm{I}_{\mathrm{Ks}}$ in canine hypertrophied hearts. Cardiovasc Res 2003;57:486-96.

10. Ramakers C, Stengl M, Spätjens RLHMG, Moorman AFM, Vos MA. Molecular and electrical characterization of the canine cardiac ventricular septum. J Mol Cell Cardiol 2005;38:153-61.

11. Lei M, Cooper PJ, Camelliti P, Kohl P. Role of the 293b-sensitive, slowly activating delayed rectifier potassium current, $\mathrm{I}_{\mathrm{Ks}}$ in pacemaker activity of rabbit isolated sino-atrial node cells. Cardiovasc Res 2002;53:68-79.

12. Han W, Wang Z, Nattel S. Slow delayed rectifier current and repolarization in canine cardiac Purkinje cells. Am J Physiol 2001;280: H1075-80.

13. Volders PGA, Stengl M, van Opstal JM, Gerlach U, Spätjens RLHMG, Beekman JDM, et al. Probing the contribution of $\mathrm{I}_{\mathrm{Ks}}$ to canine ventricular repolarization: key role for $B$-adrenergic receptor stimulation. Circulation 2003;107:2753-60.

14. Jost N, Virág L, Bitay M, Takács J, Lengyel C, Biliczki P, et al. Restricting excessive cardiac action potential and QT prolongation: a vital role for $\mathrm{I}_{\mathrm{Ks}}$ in human ventricular muscle. Circulation 2005;112: 1392-9. 
15 Shimizu W, Antzelevitch C. Cellular basis for the ECG features of the LQT1 form of the long-QT syndrome: effects of $B$-adrenergic agonists and antagonists and sodium channel blockers on transmural dispersion of repolarization and torsade de pointes. Circulation 1998;98:2314-22.

16. Shimizu W, Antzelevitch C. Differential effects of beta-adrenergic agonists and antagonists in LQT1, LQT2 and LQT3 models of the long QT syndrome. J Am Coll Cardiol 2000;35:778-86.

17. Van de Water A, Verheyen J, Xhonneux R, Reneman RS. An improved method to correct the QT interval of the electrocardiogram for changes in heart rate. J Pharmacol Methods 1989;22:207-17.

18. Lekanne Deprez RH, Fijnvandraat AC, Ruijter JM, Moorman AFM. Sensitivity and accuracy of quantitative real-time polymerase chain reaction using SYBR green I depends on cDNA synthesis conditions. Anal Biochem 2002;307:63-9.

19. Ramakers C, Ruijter JM, Lekanne Deprez RH, Moorman AFM. Assumption-free analysis of quantitative real-time polymerase chain reaction (PCR) data. Neurosci Lett 2003;339:62-6.

20. Sanguinetti MC, Jurkiewicz NK. Role of external $\mathrm{Ca}^{2+}$ and $\mathrm{K}^{+}$in gating of cardiac delayed rectifier $\mathrm{K}^{+}$ currents. Pflügers Arch 1992;420:180-6.

21. Thomas GP, Gerlach U, Antzelevitch C. HMR 1556, a potent and selective blocker of slowly activating delayed rectifier potassium current. J Cardiovasc Pharmacol 2003;41:140-7.

22. Donker DW, Volders PGA, Arts T, Bekkers BCAM, Hofstra L, Spätjens RLHMG, et al. End-diastolic myofiber stress and ejection strain increase with ventricular volume overload: serial in-vivo analyses in dogs with complete atrioventricular block. Basic Res Cardiol 2005;100:372-82.

23. Sorota S, Siegal MS, Hoffman BF. The isoproterenol-induced chloride current and cardiac resting potential. J Mol Cell Cardiol 1991;23:1191-8.

24. Fülöp L, Fiák E, Szentandrássy N, Magyar J, Nánási PP, Bányász T. The role of transmembrane chloride current in afterdepolarisations in canine ventricular cardiomyocytes. Gen Physiol Biophys 2003;22: 341-53.

25. Tsuji $Y$, Opthof T, Yasui $\mathrm{K}$, Inden $\mathrm{Y}$, Takemura $\mathrm{H}$, Niwa N, et al. Ionic mechanisms of acquired QT prolongation and torsades de pointes in rabbits with chronic complete atrioventricular block. Circulation 2002;106:2012-8.

26. Xu X, Rials SJ, Wu Y, Salata JJ, Liu T, Bharucha DB, et al. Left ventricular hypertrophy decreases slowly but not rapidly activating delayed rectifier potassium currents of epicardial and endocardial myocytes in rabbits. Circulation 2001;103:1585-90.

27. Li GR, Lau CP, Leung TK, Nattel S. Ionic current abnormalities associated with prolonged action potentials in cardiomyocytes from diseased human right ventricles. Heart Rhythm 2004;1:460-8.

28. Li GR, Sun H, Feng J, Nattel S. Ionic mechanisms of the action potential prolongation in failing human ventricular cells. Pacing Clin Electrophysiol 1998;21:877 Abstract.

29. Wang W, Xia J, Kass RS. MinK-KvLOT1 fusion proteins, evidence for multiple stoichiometries of the assembled IsK channel. J Biol Chem 1998;273:34069-74.

30. Yang T, Kanki H, Roden DM. Phosphorylation of the $I_{\mathrm{Ks}}$ channel complex inhibits drug block: novel mechanism underlying variable antiarrhythmic drug actions. Circulation 2003;108:132-4. 
31. Dilly KW, Kurokawa J, Terrenoire C, Reiken S, Lederer WJ, Marks AR, et al. Overexpression of B2-adrenergic receptors CAMP-dependent protein kinase phosphorylates and modulates slow delayed rectifier potassium channels expressed in murine heart: evidence for receptor/ channel co-localization. J Biol Chem 2004;279:40778-87.

32. Kathöfer S, Zhang W, Karle C, Thomas D, Schoels W, Kiehn J. Functional coupling of human B3-adrenoreceptors to the KvLOT1/MinK potassium channel. J Biol Chem 2000;275:26743-7.

33. Bosch RF, Schneck AC, Kiehn J, Zhang W, Hambrock A, Eigenberger BW, et al. B3-Adrenergic regulation of an ion channel in the heart-inhibition of the slow delayed rectifier potassium current $I_{\mathrm{Ks}}$ in guinea pig ventricular myocytes. Cardiovasc Res 2002;56:393-403.

34. Marx SO, Kurokawa J, Reiken S, Motoike H, D'Armiento J, Marks AR, et al. Requirement of a macromolecular signaling complex for $ß$ adrenergic receptor modulation of the KCNQ1-KCNE1 potassium channel. Science 2002;295:496-9.

35. Jurevicius J, Fischmeister R. cAMP compartmentation is responsible for a local activation of cardiac $\mathrm{Ca}^{2+}$ channels by B-adrenergic agonists. Proc Natl Acad Sci U S A 1996;93:295-9.

36. Schwartz PJ, Priori SG, Spazzolini C, Moss AJ, Vincent GM, Napolitano C, et al. Genotype-phenotype correlation in the long-QT syndrome: gene-specific triggers for life-threatening arrhythmias. Circulation 2001;103:89-95.

37. Shimizu W, Noda T, Takaki H, Kurita T, Nagaya N, Satomi K, et al. Epinephrine unmasks latent mutation carriers with LQT1 form of congenital long-QT syndrome. J Am Coll Cardiol 2003;41:633-42. 
Dirk W. Donker, Jos G. Maessen, Fons Verheyen, Frans C. Ramaekers, Roel L.H.M.G. Spätjens, Helma Kuijpers, Christian Ramakers, Paul M.H. Schiffers, Marc A. Vos, Harry J.G.M. Crijns, Paul G.A. Volders.

Am J Physiol Heart Circ Physiol. 2007;292:H2324-32

Circulation. 2005;112:U467-U467, 1982 Suppl. S.

J Mol Cell Cardiol. 2002;34:A20-A20 


\section{Impact of acute and}

enduring volume overload on mechanotransduction and cytoskeletal integrity of canine left ventricular myocardium 


\section{ABSTRACT}

It is poorly understood how mechanical stimuli influence in vivo myocardial remodeling during chronic hemodynamic overload. Combined quantitation of ventricular mechanics and expression of key proteins involved in mechanotransduction can improve fundamental understanding. Adult anesthetized dogs $(n=20)$ were studied at sinus rhythm (SR) and $0,3,10$, and 35 days of complete atrioventricular block (AVB). Serial left ventricular (LV) myofiber mechanics were measured. Repeated LV biopsies were analyzed for mRNA and/or protein expression of $B_{10}$-integrin, melusin, Akt, GSK3B, muscle LIM protein (MLP), four-and-a-half LIM protein (fhl2), desmin, and calpain. Upon AVB, increased ejection strain $(0.29 \pm 0.01$ vs. $0.13 \pm 0.02, S R)$ and end-diastolic stress $(4.8 \pm 1.1$ vs. $2.7 \pm 0.4 \mathrm{kPa}$ ) dominated mechanical changes. Brain natriuretic peptide plasma levels were correspondingly high $(33 \pm 4$ vs. $19 \pm 1 \mathrm{pg} / \mathrm{ml}, \mathrm{SR})$. $B_{10}$-Integrin protein expression increased chronically after AVB. Melusin was temporarily overexpressed ( $+33 \pm 9 \%, 3$ days AVB vs. SR), followed by elevated ratios of phosphorylated (P)-Akt to Akt and P-GSK3B to GSK3B $(+26 \pm 6 \%$ and $+30 \pm 8 \%$ at 10 days AVB vs. SR). These changes corresponded to peak hypertrophic growth at 3 to 10 days. MLP increased gradually to maxima at chronic AVB $(+36 \pm 7 \%)$. In contrast, fhl $2(-22 \pm 3 \%, 3$ days $)$ and desmin $(-30 \pm 9 \%, 10$ days AVB) transiently declined but recovered at chronic AVB. Calpain protein expression remained unaltered.

In conclusion, volume overload after AVB causes a transient compromise of cytoskeletal integrity based, at least partly, on transcriptional downregulation. Subsequent cytoskeletal reorganization coincides with the upregulation of melusin, P-Akt, P-GSK3B and MLP, indicating strong drive to compensated hypertrophy. 


\section{INTRODUCTION}

It is still poorly understood how mechanical signals are sensed by the myocardium and transduced into cellular-biological responses. Mechanical load imposed on the myocardium can be dissected into stress, which is the force per cross-sectional area of tissue, and strain, the resultant cellular deformation. Stress and strain exhibit cyclic beat-to-beat variations in the normal heart, and they are both stimuli for and responders to remodeling induced by pathological overload.

Recently, we described the time course of left ventricular (LV) myocardial mechanics in dogs with bradycardia-induced volume overload due to complete atrioventricular block (AVB) [11]. Early after $A V B$, increased end-diastolic myofiber stress and ejection strain are imposed on the myocardium, and they coincide with peak hypertrophic growth, suggesting their role as primary stimuli for mechanotransduction.

Besides ventricular hypertrophy, the AVB model is characterized by important electrical, contractile, and structural remodeling, yet an exact relation with mechanical overload is currently unknown. In the present study, we examined the time-dependent effects of increased load on myocardial remodeling during $A V B$, focusing specifically on the expression of key proteins involved in mechanotransduction and cytoskeletal integrity, i.e., laminin, $B_{1 D}$-integrin, $B_{1 D}$-integrin-interacting protein melusin, Akt (protein kinase B), GSK3B, muscle LIM protein (MLP), four-and-a-half LIM protein 2 (fhl2), and desmin. Serial analysis of myofiber mechanics was combined with the determination of plasma levels of brain natriuretic peptide (BNP), a biochemical marker of load. Repetitive sampling of intramural LV biopsies was applied to analyze protein expressions in individual dogs over time.

\section{METHODS}

Experiments were conducted in accordance with the 'European Directive for the Protection of Vertebrate Animals Used for Experimental and Other Scientific Purposes (86/609/EU)'. The Committee for Experiments on Animals of Maastricht University approved the experiments.

\section{Model of complete atrioventricular block}

Twenty adult mongrel dogs of either sex weighing $25 \pm 1 \mathrm{~kg}$ were used. After dogs were fasted overnight, premedication $(1 \mathrm{ml} / 10 \mathrm{~kg}$ i.m. Acetadon containing $1.5 \mathrm{mg} / \mathrm{ml}$ acepromazine, $4 \mathrm{mg} / \mathrm{ml}$ methadone, and $0.6 \mathrm{mg} / \mathrm{ml}$ atropine) was administered. Complete anesthesia was induced by thiopental sodium (20 mg/kg iv) and maintained with halothane (0.5-1\%) and $\mathrm{O}_{2}-\mathrm{N}_{2} \mathrm{O}(1: 2)$. AVB was induced by radio-frequency catheter ablation of the His bundle. Animals were studied serially at sinus rhythm (SR; control) and at 0, 3, 10, and 35 days AVB. A standard six-lead ECG was registered. After the animals were killed ( 35 days AVB), the hearts were excised and weighed. 


\section{LV mechanics}

LV mechanics were quantified as myofiber stress and strain by applying serial transthoracic echocardiography synchronized with recordings of LV cavity pressure using a validated mathematical model [2], as we have recently described in detail [11]. Plasma levels of BNP were determined in peripheral venous blood, collected in chilled EDTA tubes, centrifuged $\left(1,600 \mathrm{~g}, 10 \mathrm{~min}, 4^{\circ} \mathrm{C}\right)$, and stored at $-80^{\circ} \mathrm{C}$ until measured in duplicate by a radioimmunoassay specific for canine BNP-32 (Phoenix Pharmaceuticals, Mountain View, CA).

\section{Serial percutaneous sampling of LV intramural biopsies}

Intramural LV needle biopsies were taken serially in dogs at SR and after 3, 10, and 35 days AVB. A 16-gauge biopsy needle (Acecut, TSK Laboratory) was percutaneously inserted into the apicolateral LV wall, under fluoroscopic guidance. At least two samples $\left(\sim 10 \mathrm{~mm}^{3}\right)$ were obtained per experiment. Tissue quality was optimal in most biopsies (>98\%). All animals were followed by transthoracic echocardiography after biopsy sampling to exclude cardiac tamponade, although in our experience the closed-chest approach is generally uncomplicated (>95\%) and fatal outcome is rare $(<3 \%)$. Such complications did not occur in this study.

\section{Light and electron microscopy}

For light (LM) and electron microscopy (EM), biopsies were processed and analyzed as described before [4]. Briefly, tissue was fixed in 3\% glutaraldehyde and embedded in epoxy resin. LM analysis was performed on periodic acid-Schiff (PAS)-/Toluidine blue-stained sections to depict cardiomyocyte glycogen content, sarcomeres, mitochondria, and nuclei. PAS positivity and absence of contractile filaments in $>10 \%$ of the cell surface area of transversely sectioned cardiomyocytes were considered abnormal.

\section{Immunohistochemistry}

Indirect immunohistochemistry was performed on briefly fixed tissue (3\% glutaraldehyde or 3.7\% formaldehyde) using primary antibodies directed against desmin (DE-R-11, 1:10) (Dako Cytomation, Glostrup, Denmark), MLP (1:80) [1], vimentin (K36, 1:10) (Frans C. Ramaekers, Maastricht University), and laminin (L9393, 1:150) (Sigma, St. Louis, MO). Specific labeling was visualized using appropriate secondary antibodies applying immunoperoxidase or immunofluorescence techniques. Nuclei were stained using Mayers hemalum or propidium iodide. For negative control, the primary antibodies were omitted. Immunofluorescent labeling was analyzed using confocal laser scanning microscopy (Bio-Rad MRC600, Bio-Rad, Hercules, CA). 


\section{Real-time PCR}

Total RNA was isolated from the biopsies with the use of the RNEasy Mini kit (Qiagen/Westburg, Leusden, The Netherlands). Fluorescence-based kinetic real-time PCR was performed with a LightCycler system (Roche Diagnostics, Almere, The Netherlands), and amplicons were quantified relative to the constitutively active $18 \mathrm{~S}$ rRNA by using the LinRegPCR method [31].

\section{Western blotting}

Total homogenates were derived from unfixed cryosections $(5 \mu \mathrm{m})$ dissolved for $30 \mathrm{~min}$ on ice in lysis buffer (62 mmol/l Tris, $1.25 \mathrm{mmol} / /$ EDTA, 2\% Nonidet P-40, $2.5 \mathrm{mmol} / /$ phenylmethylsulfonyl chloride, $12.5 \mathrm{mg} / \mathrm{ml}$ leupeptin, $12.5 \%$ glycerol, $100 \mathrm{mg} / \mathrm{ml}$ aprotinin, $0.5 \mathrm{mM}$ sodium orthovanadate, and 2.3\% SDS) and sample buffer for $4 \mathrm{~min}$ at $95^{\circ} \mathrm{C}(62 \mathrm{mmol} / \mathrm{l}$ Tris, $2.3 \% \mathrm{SDS}, 10 \%$ glycerol, $5 \%$ B-mercaptoethanol, and $0.05 \%$ bromophenol blue). Equal amounts of total protein (7.5-30 $\mu \mathrm{g} /$ lane), determined by protein assay (Bio-Rad), were loaded on 10\% SDS-PAGE gels, transferred to nitrocellulose membranes, and controlled by Coomassie brilliant blue, Ponceau $S$ staining, and GAPDH immunoblotting. Membranes were blocked in BSA $(3 \%, 1 \mathrm{~h})$ in Tween buffer (TWB), containing PBS and Tween 20 (0.05\%), and incubated with the primary antibody in BSA $(0.5 \%, 1 \mathrm{~h})$ against desmin (DE-R-11, 1:5,000), $B_{10}$-integrin (2B1, 1:100) (Mubio, Maastricht, The Netherlands), melusin (1:500) [7], MLP (1:500) [1], fhl2 (1:500) [24], Akt (9272, 1:1000), phosphorylated (P)-Akt (Ser473, 1:1000), GSK3ß. (9315, 1:1000), P-GSK3ß. (Ser9, 1:1000) (Cell Signaling Technology, Danvers, $M A)$, and the regulatory subunit of calpain I and II (P1, 1:1100) (Chemicon, Temecula, CA). After blots were washed in TWB, they were incubated $(1 \mathrm{~h})$ using the appropriate secondary antibodies, washed again, and visualized on X-ray films (Fujifilm, Rotterdam, The Netherlands) with enhanced chemiluminescence (Amersham Biosciences, Amersham, UK). Densitometric quantification was performed with Quantity One software using a GS-800 scanner (Bio-Rad). All immunoblots were performed in duplicate.

\section{Statistical analysis}

Data are presented as means \pm SE. Data were compared by using Student's t-test for unpaired or paired data. Serial data were tested by repeated-measures ANOVA using Bonferroni's post test comparison. Differences were considered statistically significant if $\mathrm{P}<0.05$. 


\section{RESULTS}

\section{Temporal aspects of mechanical overload and BNP expression}

For information regarding hemodynamic changes after AVB in this study, we refer to Table 1. Consistent with our previous study [11], LV mechanical load was characterized by early increases of end-diastolic myofiber stress ( $4.8 \pm 1.1$ vs. $2.7 \pm 0.4 \mathrm{kPa}$ at $\mathrm{SR}, \mathrm{P}<0.01)$ and ejection strain $(0.29 \pm 0.01$ vs. $0.13 \pm 0.02$ [no units] at $S R, P<0.01$ ). End-diastolic stress normalized after 35 days AVB (Figure $1, A$ and $B)$. Systolic myofiber stress did not change during AVB (37.3 \pm 3.6 vs. $38.0 \pm 4.3 \mathrm{kPa}$ at $S R$, $P=$ not significant $(n s)$ ).

The impact of increased stress and strain on the myocardium was reflected by maximally elevated plasma levels of BNP as early as 3 days after AVB $(32.9 \pm 3.5$ vs. $19.1 \pm 1.0 \mathrm{pg} / \mathrm{ml}$ at $S R ; P<0.01)$. BNP remained high throughout the experimental period (Figure 1C). Temporal changes of plasma BNP correlated well with ejection strain $\left(r^{2}=0.75\right)$, whereas only a weak correlation with end-diastolic stress was found $\left(r^{2}=0.004\right)$ (Figure 2D).

\section{Dynamic cardiomyocyte remodeling after AVB}

The presence of cardiac hypertrophy was confirmed at autopsy after 35 days. Total heart weight $(270 \pm 12 \mathrm{~g})$ and heart weight-to-body weight ratio $(10.7 \pm 0.6 \mathrm{~g} / \mathrm{kg})$ were larger than in a matching control population of dogs with SR $(202 \pm 12 \mathrm{~g}$ and $8.0 \pm 0.4 \mathrm{~g} / \mathrm{kg}$, respectively; both $\mathrm{P}<0.05)$.

Key proteins involved in mechanotransduction.

Western blot analysis revealed a significant increase of $B_{1 D}$-integrin expression after AVB when compared with SR (Figure 2A), whereas its extracellular ligand laminin did not alter (Figure 3A). Time-dependent expression of melusin, a protein directly interacting with the cytoplasmic domain of $B_{1 D}$-integrin at the $Z$ disk, was characterized by an early peak at 3 days AVB $(+33 \pm 9 \%$ vs. SR, $\mathrm{P}<0.05$ ), which gradually declined thereafter (Figure $2 \mathrm{~B}$ ).

The P-Akt-to-Akt (P-Akt/Akt) ratio increased at 10 days AVB $(+26 \pm 6 \%$ vs. SR, $P<0.05)$ (Figure $4 A)$. The Akt substrate GSK3 $\beta$ was phosphorylated along with Akt, reaching peak values of the P-GSK3 $\beta$-to-GSK3 $\beta$ (P-GSK3 $\beta / G S K 3 \beta)$ ratio at 10 days $(+30 \pm 8 \%$ vs. SR, $P<0.05)$ (Figure 4B). This higher degree of Akt and GSK3 $\beta$ phosphorylation was transient, because the P-Akt/Akt and P-GSK3B/GSK3B ratios at 35 days AVB were not different from control.

The cardiac LIM domain proteins MLP and fhl2, which both act at the $Z$ disk downstream from $B_{1 D}$-integrin-melusin, showed divergent expression patterns during AVB. MLP gradually increased to maxima at chronic AVB $(+36 \pm 7 \%$ at 35 days vs. $S R, P<0.01$; Figure $2 C)$, whereas fhl2 exhibited an early decline $(-22 \pm 3 \%$ vs. $S R, P<0.01)$ (Figure $2 D)$. Immunohistochemical studies revealed 
Table 1 Hemodynamic and electrocardiographic measurements

\begin{tabular}{llllll}
\hline & SR & AAVB & 3 days & 10 days & 35 days \\
\hline LV pressure & & & & & \\
Peak systole, $\mathrm{kPa}$ & $12.5 \pm 1.1$ & $10.0 \pm 0.7$ & $12.4 \pm 2.0$ & $12.1 \pm 0.6$ & $12.3 \pm 0.8$ \\
End diastole, $\mathrm{kPa}$ & $0.8 \pm 0.1$ & $1.5 \pm 0.3^{\star}$ & $1.3 \pm 0.2$ & $1.1 \pm 0.2$ & $0.8 \pm 0.1$ \\
Maximum rise, $\mathrm{kPa} / \mathrm{s}$ & $142 \pm 9$ & $141 \pm 20$ & $244 \pm 44^{\star}$ & $238 \pm 21^{\star}$ & $236 \pm 41^{\star}$ \\
Heart rate, beats/min & $107 \pm 5$ & $44 \pm 9^{*}$ & $45 \pm 4^{\star}$ & $46 \pm 4^{\star}$ & $48 \pm 4^{\star}$ \\
Cardiac output, $/ \mathrm{min}$ & $3.1 \pm 0.4$ & $1.4 \pm 0.4^{*}$ & $1.8 \pm 0.3$ & $2.0 \pm 0.3$ & $2.2 \pm 0.1$ \\
Ejection fraction, $\%$ & $48 \pm 4$ & $62 \pm 5$ & $70 \pm 2^{\star}$ & $77 \pm 3^{\star}$ & $75 \pm 3^{\star}$ \\
\hline
\end{tabular}

Values are means \pm SE. SR, sinus rhythm (control); AAVB, acute atrioventricular block; LV, left ventricular. ${ }^{*} P<0.05$ vs. SR.

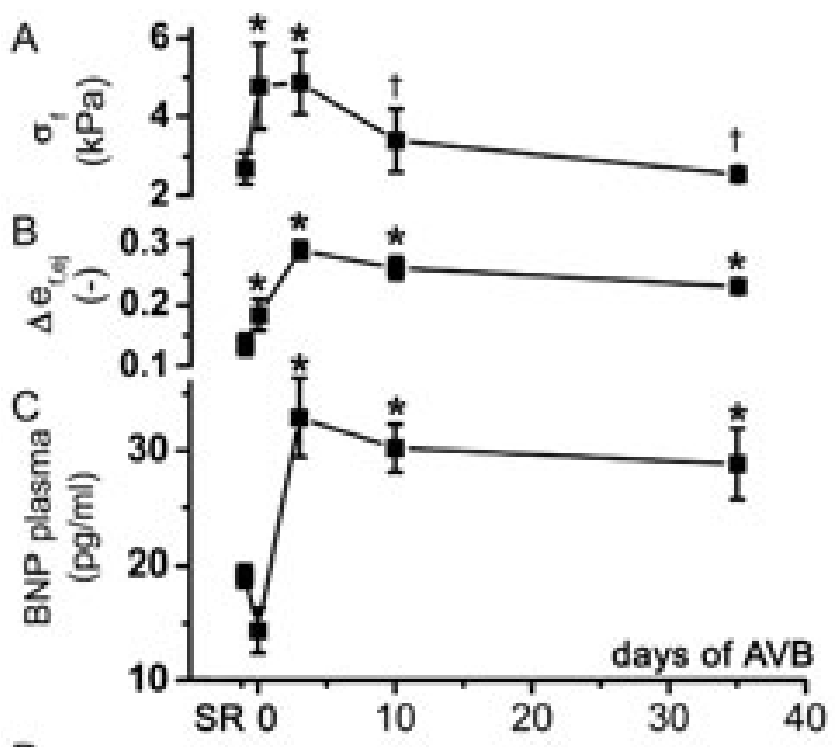

D
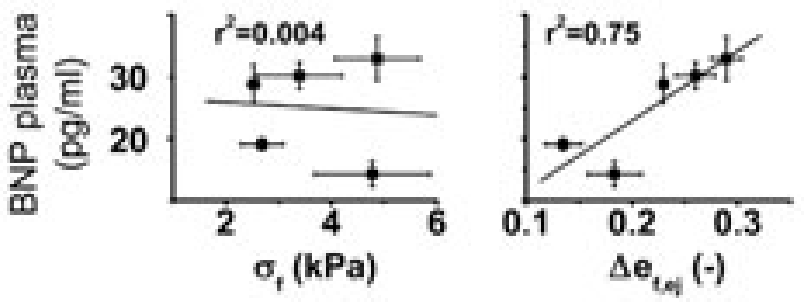

Figure 1 | Time course of mechanical overload before and after atrioventricular block (AVB). Average curves ( $n=5$ dogs) of end-diastolic stress $\left(\sigma_{f}\right)$ A, ejection strain $\left(\Delta \mathrm{e}_{\mathrm{t}, \mathrm{e}}\right) \mathbf{B}$, and plasma levels of brain natriuretic peptide (BNP) $\mathbf{C}$ are shown. Changes of ejection strain D (right), and not of end-diastolic stress D (left), correlate well with altering BNP plasma levels. ${ }^{*} P<0.05$ vs. sinus rhythm (SR); $\uparrow P<0.05$ vs. 3 days AVB. 

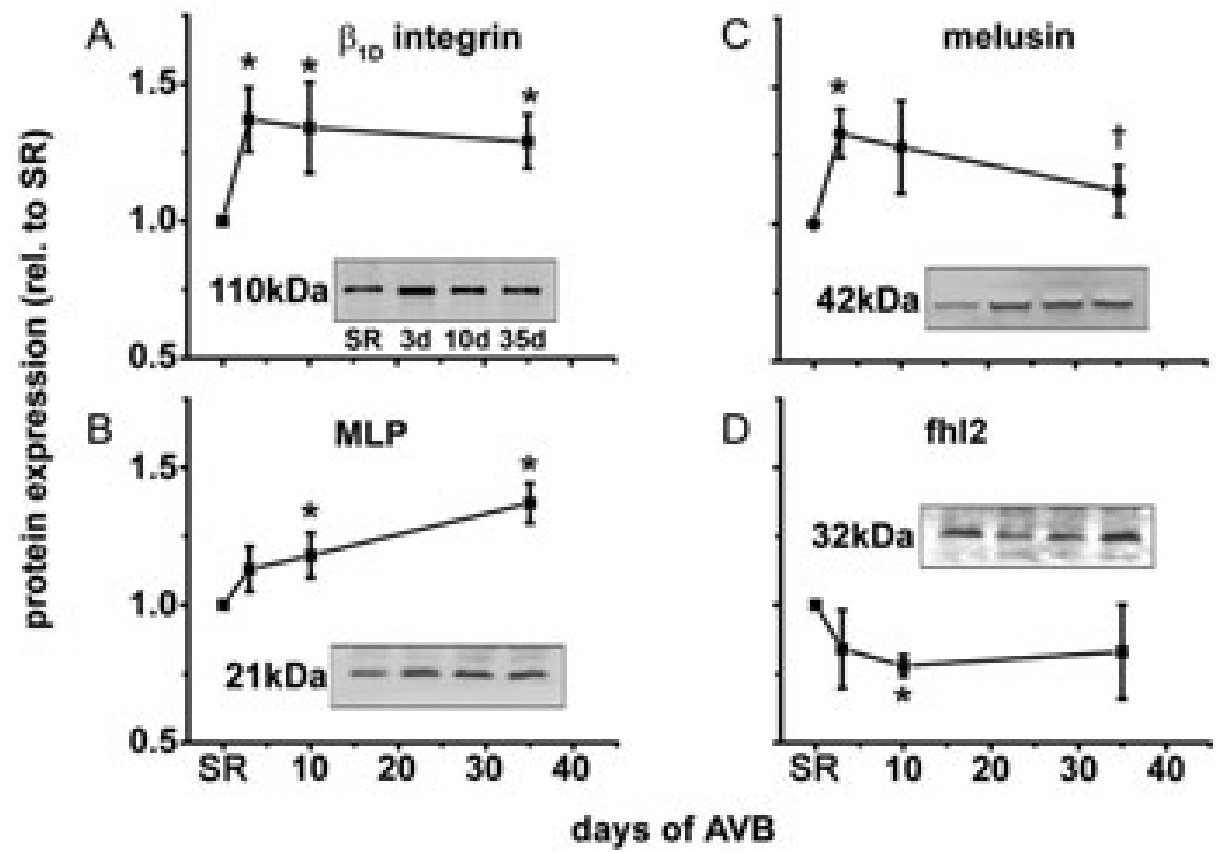

Figure 2 | Time course of Z-disk protein expression before and after AVB. Serial Western blot analysis of $B_{10}$-integrin $\mathbf{A}$, melusin $\mathbf{B}$, muscle LIM protein (MLP) C, and four-and-a-half LIM protein 2 (fhl2) $\mathbf{D}$ is shown. Average values ( $\mathrm{n}=7$ dogs) are standardized to SR; rel, relative. ${ }^{*} P<0.05$ vs. $S R ; \uparrow P<0.05$ vs. 3 days (3d) AVB.

that, during AVB, MLP expression increased throughout the cytoplasm and was also prominently expressed in the nucleus (Figure 5). The temporal expression of MLP correlated highly with increasing echographic LV mass (in grams) over the weeks after AVB (Figure 6). In addition, we found a good correlation between the expression of melusin, the ratiometric expression of P-Akt/Akt and P-GSK3B/GSK3B, and the degree of hypertrophic growth (LV mass increase per time in $\mathrm{g} /$ day; Figure 6).

Time course of cytoskeletal and sarcomeric changes.

(Immuno)histological analysis revealed a normal compact myocardial texture and no alteration of the extracellular space during AVB (Figure 3) in line with earlier work [37]. The extracellular space was characterized by unaltered expressions of the $B_{10}$-integrin-interacting extracellular protein laminin (Figure $3 \mathrm{~A}$ ) and the fibroblast intermediate filament vimentin (Figure 3B). Micromorpho- 

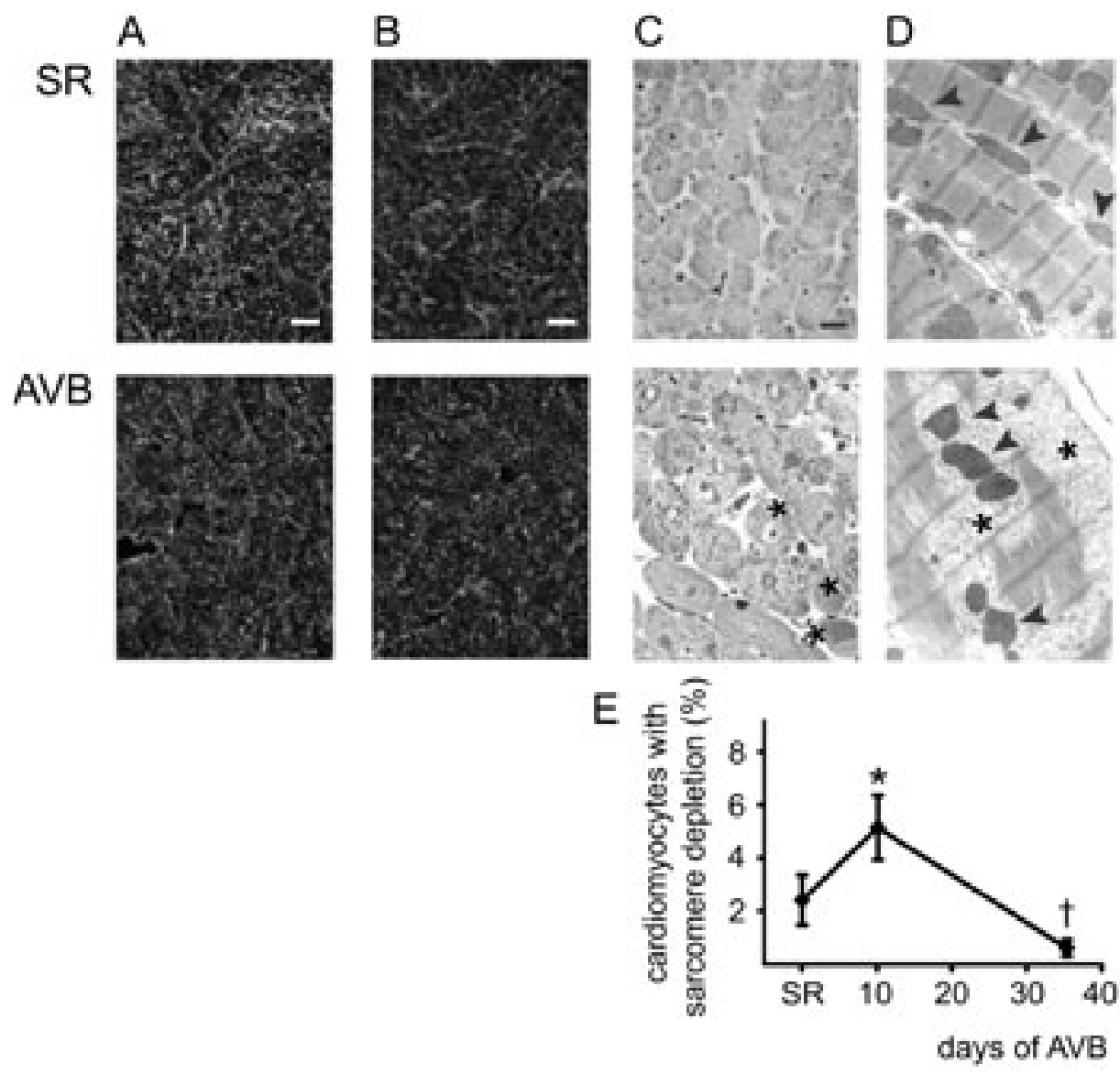

Figure $3 \mid$ Time course of structural remodeling before and after AVB. Immunofluorescent double staining for desmin (green) and laminin (red) A and desmin (green) and vimentin (red) B showing no differences between SR and AVB. C light microscopy (LM) photomicrographs of periodic acid-Schiff-/Toluidine blue-stained left ventricular (LV) myocardium from serial biopsies of one individual dog showing increased cytoplasmic glycogen content during AVB (asterisks), which was absent at SR. D electron microscopy photomicrographs illustrating ultrastructural details, as glycogen accumulation in areas with depletion of sarcomeres and remnants of myofibrils (asterisks) altered mitochondrial shape and size as compared with SR (arrowheads). E relative number of cardiomyocytes showing depletion of sarcomeres. Scale bars in $A-C$ indicate $10 \mu \mathrm{m}$. ${ }^{*} \mathrm{P}<0.05$ vs. $S R ; \mathrm{P}<0.05$ vs. 10 days AVB. For full colour illustration, see page 226. 

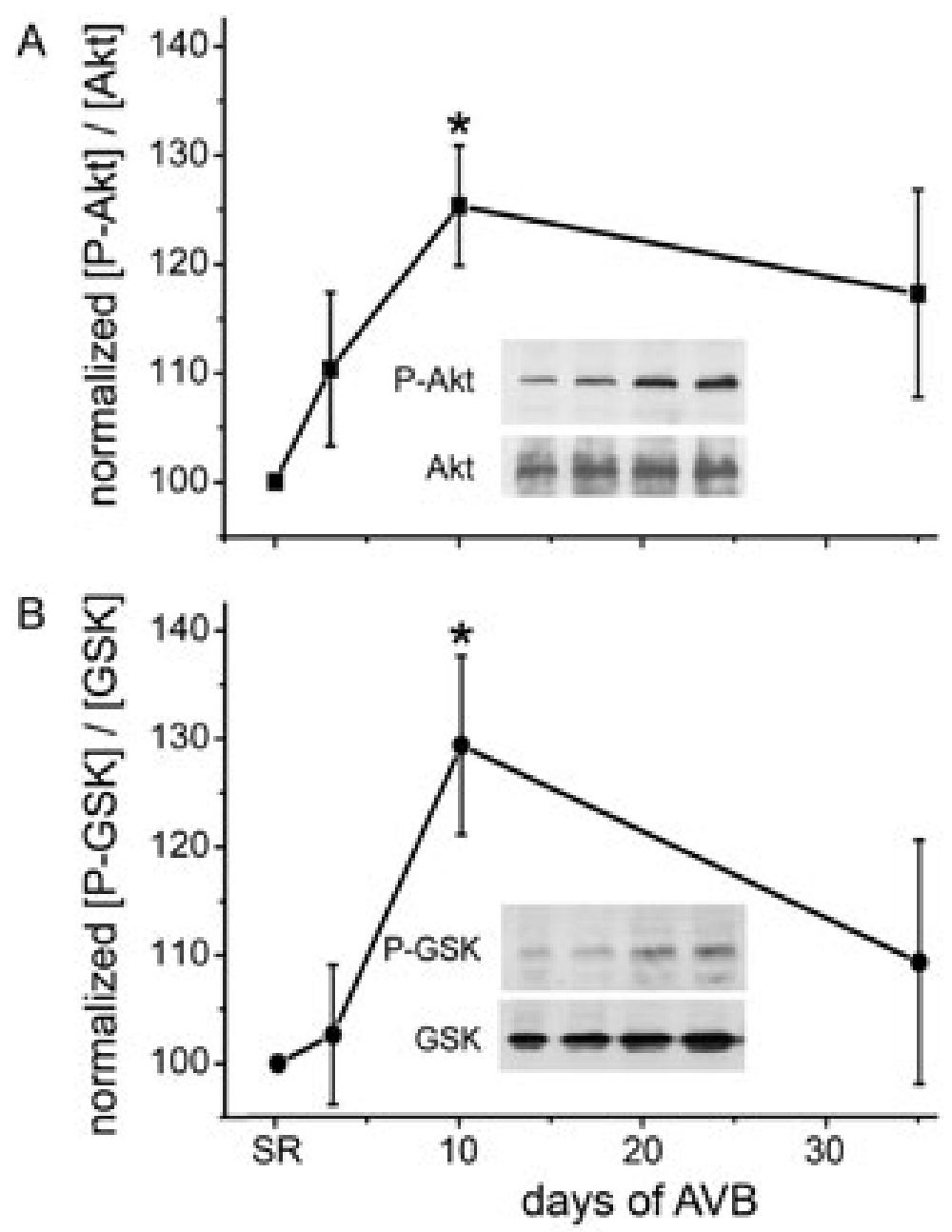

Figure $4 \mid$ Time course of Akt and GSK3B protein expression and phosphorylation before and after AVB. Serial Western blot analysis and normalized ratio of phosphorylated $(P)$ to total protein expression of Akt $\mathbf{A}$ and GSK3B B are shown. Average values ( $n=5$ dogs) are standardized to $S R$. ${ }^{*} P<0.05$ vs. SR. 


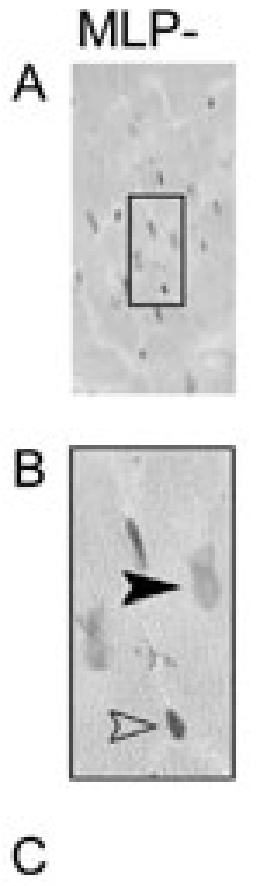

\section{SR}
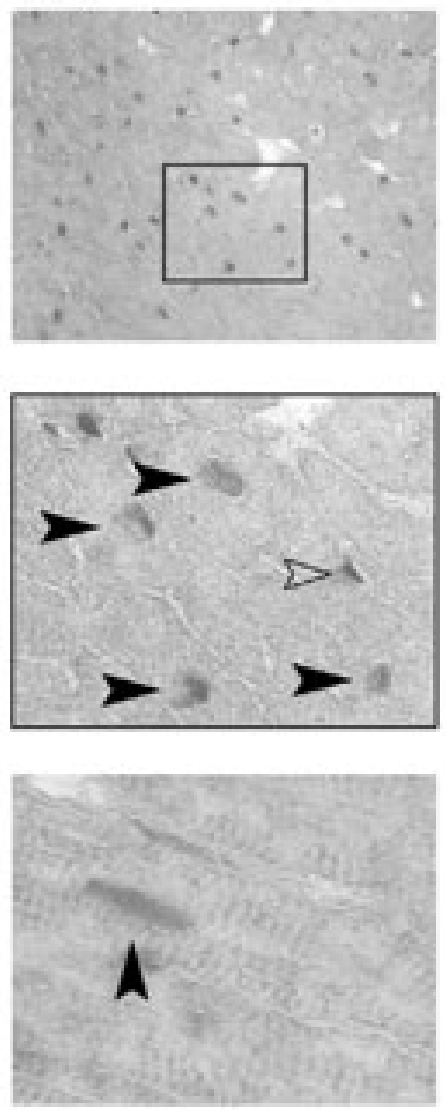

AVB
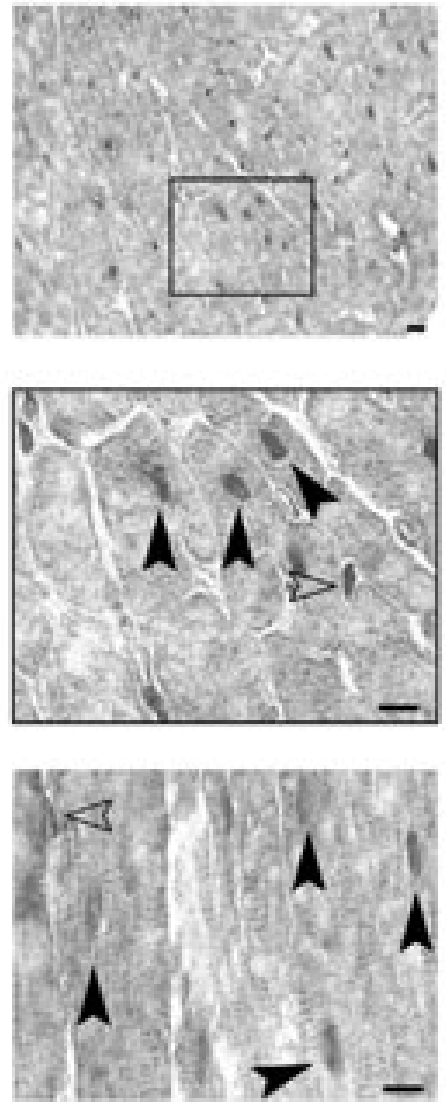

Figure $5 \mid$ Cardiomyocyte expression pattern of MLP before and after AVB. LM photomicrographs show immunohistochemical expression of MLP in LV myocardium at low $\mathbf{A}$ and high $\mathbf{B}$ and $\mathbf{C}$ magnification, in transversely $\mathbf{A}$ and $\mathbf{B}$ and longitudinally $\mathbf{C}$ sectioned cardiomyocytes. At control (SR), a mild MLP expression was observed within the cytoplasm and the nucleus B and C; solid arrowheads. Note that noncardiomyocyte nuclei B and C, open arrowhead stain blue, whereas cardiomyocyte nuclei show MLP labeling B and C, solid arrowhead. In negative control (MLP.), both cardiomyocyte nuclei $\mathbf{B}$ and $\mathbf{C}$, solid arrowhead and noncardiomyocyte nuclei $\mathbf{B}$ and $\mathbf{C}$, open arrowheads stain blue, indicating the absence of MLP labeling. During AVB, both cardiomyocyte cytoplasmic and nuclear expression of MLP are increased B and C. Scale bars indicate $10 \mu \mathrm{m}$. For full colour illustration, see page 227. 
A
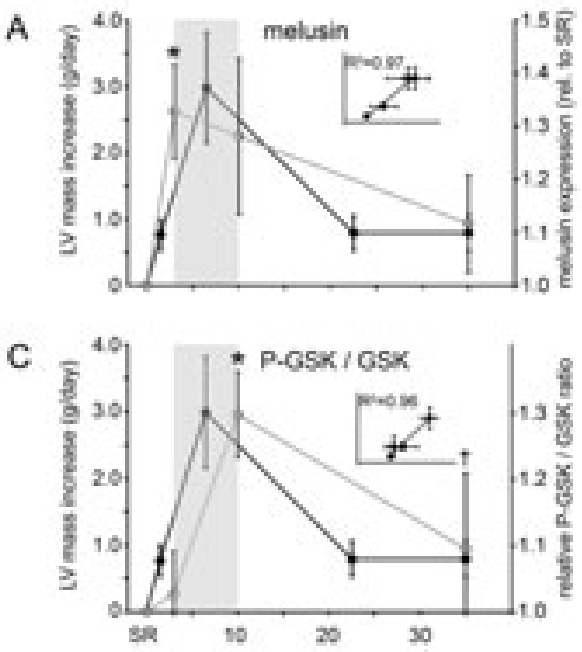

B

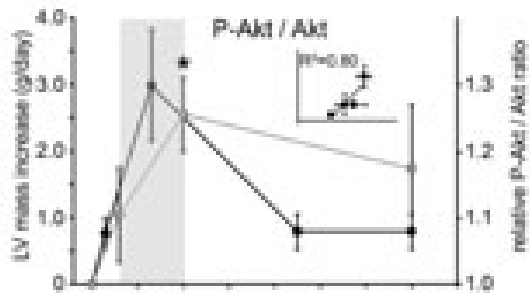

D

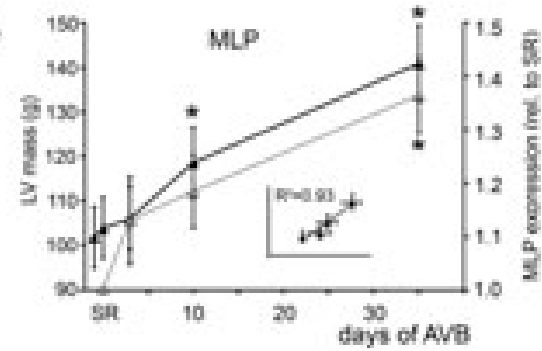

Figure 6 | Temporal expression of melusin, P-Akt/Akt, P-GSK3 $\beta /$ GSK3 $\beta$, and MLP related to LV hypertrophy. The time course of melusin, P-Akt/Akt, and P-GSK3 $3 /$ GSK3 $\beta$ expression A-C, shaded lines correlated (insets) highly with the echocardiographically determined increase of LV mass per time, a measure of hypertrophic growth $\mathbf{A}-\mathbf{C}$, solid lines, whereas the time course of MLP expression $\mathbf{D}$, shaded line correlated (inset) highly with the time course of the echographic LV mass after AVB D, solid line ( $n=5$ dogs). Shaded bars $\mathbf{A}-\mathbf{C}$ indicate time interval of peak hypertrophic growth. ${ }^{*} P<0.05$ vs. $S R ; ~ † P<0.05$ vs. 3 days AVB.

metry of cardiomyocyte cross sections showed comparable diameters between SR $(23 \pm 1 \mu \mathrm{m})$, 10 days $(24 \pm 1 \mu \mathrm{m}, \mathrm{P}=\mathrm{ns})$, and 35 days $A V B(25 \pm 1 \mu \mathrm{m}, \mathrm{P}=\mathrm{ns})$, supporting previous data showing predominant cellular lengthening after AVB $[11,37]$.

Cytoskeletal integrity was further explored by analysis of the intermediate filament desmin (Figure 7). During SR, quantitative analysis of mRNA and protein expression showed a downregulation at 3 and 10 days AVB and subsequent normalization (Figure 7, A and B). Desmin immunolabeling was in agreement with the mRNA and protein data and exhibited a normal cross-striated pattern at the $Z$ line and prominent expression at the intercalated disk. At 3 and 10 days AVB, desmin labeling at the intercalated disk clearly decreased compared with the Z-line labeling. After 35 days, desmin expression was restored, particularly at the intercalated disks (Figure 7, C and D).

To determine whether the early decrease of desmin was also reflected by derangements of sarcomeric structures, we analyzed the cardiomyocyte ultrastructure by EM (Figure 3D). We found cytoplasmic areas largely devoid of sarcomeric structures, notably at 10 days AVB. These areas exhibited remnants of myofibrils and were filled with glycogen and with healthy-appearing, abnormally large-and small-sized mitochondria. We did not observe mitochondrial swelling, loss of intra- 


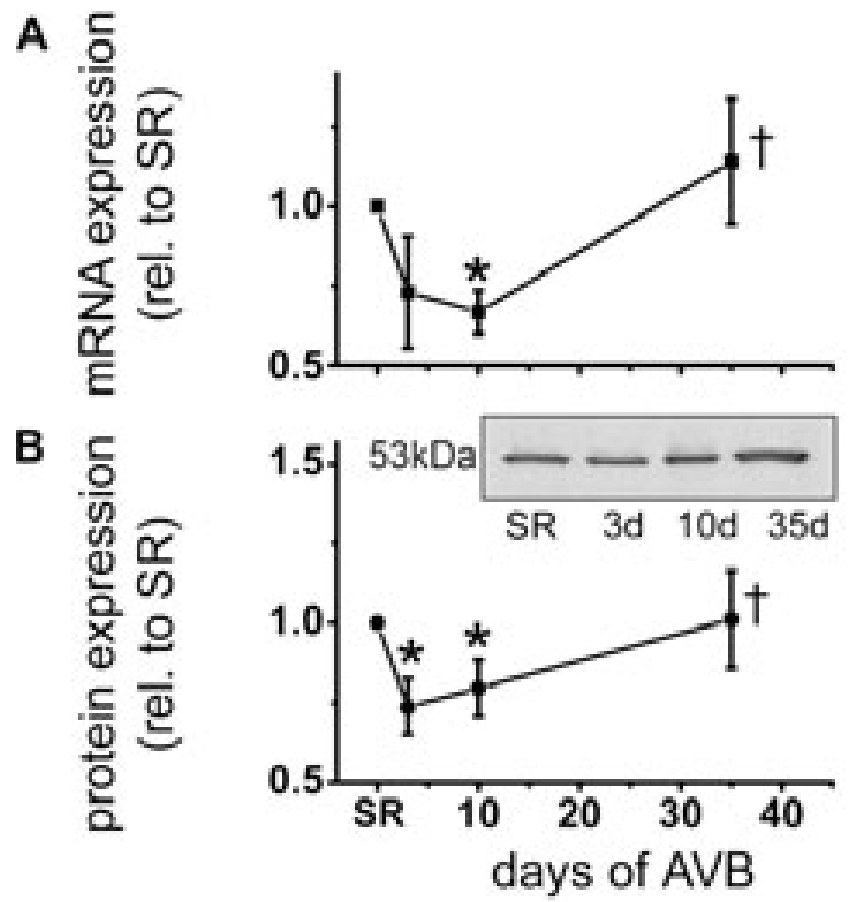

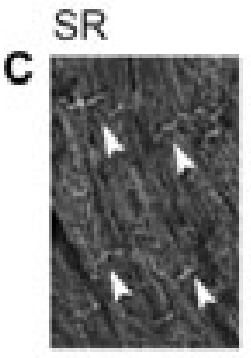
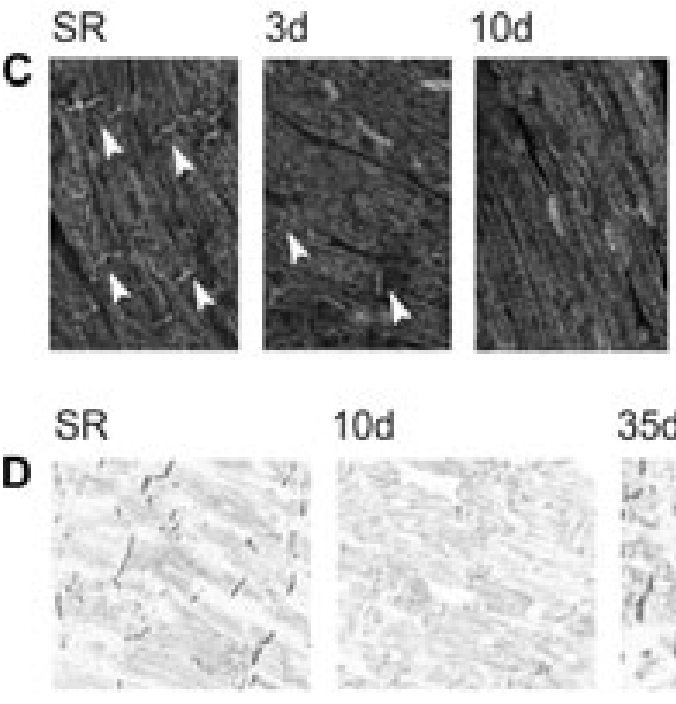

10d

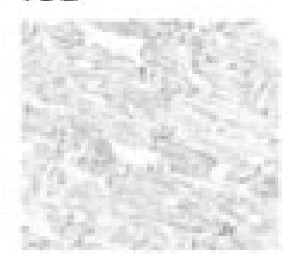

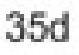

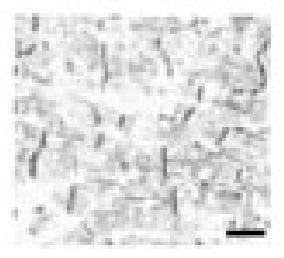

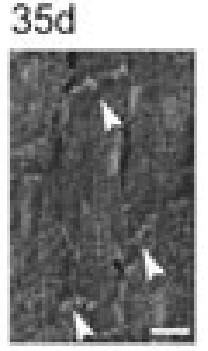

Figure $7 \mid$ Time course of desmin expression before and after AVB. Serial real-time PCR $\mathrm{a}$ and Western blot analysis b show average values standardized to $S R(n=9$ dogs). Immunofluorescent labeling of desmin (green) and nuclei (red) c and LM photomicrographs $\mathbf{d}$ show immunohistochemical expression of desmin in serial biopsies. Scale bars indicate $25 \mu \mathrm{m} .{ }^{*} \mathrm{P}<0.05$ vs. SR; $\uparrow \mathrm{P}<0.05$ vs. 3 days AVB. For full colour illustration, see page 228. 
matricial granules, disrupted mitochondrial cristae, mega-mitochondria with lipid-like inclusion, cytoplasmic vacuolization or edema, tortuous nuclei, intramatricial glycogen clumps, membrane disruption, or whorl-like myelin structures indicating the absence of ischemia or degeneration during AVB [4] (Figure 3D).

To quantify these abnormalities by LM, we performed PAS staining to depict the glycogen accumulation observed during EM analysis. The number of abnormal glycogen-stuffed cardiomyocytes per microscopic section area was increased at 10 days AVB $(5 \pm 2 \%$ vs. $2 \pm 1 \%$ at $S R, P<0.05)$ and had normalized again at 35 days AVB (Figure 3E).

Finally, to examine whether the $\mathrm{Ca}^{2+}$-dependent protease calpain could be involved in the loss of cytoskeletal and contractile elements, serial Western blot analyses of the regulatory subunits of calpain I and II were performed, which showed an unaltered protein expression throughout the experimental period.

\section{DISCUSSION}

\section{Time course of myofiber mechanics and plasma BNP}

In the present study we have confirmed previous results [11] that both end-diastolic stress and ejection strain are driven to maximal amplitudes within the first week of AVB. As a first new finding, this acute and enduring volume overload is reflected by a parallel release of the load biomarker BNP. Absolute plasma levels of BNP after AVB were comparable to those in dogs with compensated overload of different cause, varying roughly between 25 [16] and $40 \mathrm{pg} / \mathrm{ml}$ [3]. Correlation analysis suggests that BNP release after AVB is mainly driven by systolic ejection strain. In vitro studies support that mechanical stimuli applied during systole can be even more capable of increasing BNP transcription than diastolic stimuli [40]. Nevertheless, diastolic load may well contribute to the BNP release after AVB, despite a weak temporal correlation. Diastolic mechanical stimuli have been shown to trigger BNP release in other animal models [15] and in humans with overload [25].

\section{Dynamic expression of key mechanotransduction proteins after AVB}

Key proteins located at the Z-disk level have been proposed to sense and transduce mechanical stimuli $[13,22,30]$. Among those, integrins are obvious candidates that span the sarcolemma [32]. In cardiomyocytes, $B_{10}$-integrin is dominantly expressed [36] and has been shown to be relevant for mechanotransduction under basal (i.e., normal load) conditions and pathological overload [33]. Its increased expression has been observed during hypertrophy after adrenergic stimulation in vitro $[28,32]$ and during chronic aortic constriction in mice [5]. During $A V B, B_{1 D}$-integrin expression 
was increased from 3 days onward. This early and sustained upregulation is in line with findings in other models of hypertrophy $[5,28,32]$. Interestingly, the immunoexpression of laminin, the primary extracellular $\beta_{10}$-integrin ligand [32], and vimentin, a fibroblast marker, were found unaltered (Figure 3B). These findings suggest that the cardiomyocyte protein $\beta_{10}$-integrin, and not components of the extracellular matrix, is among the first upstream elements ("initiators") activated by mechanical stimuli in this model.

At its cytoplasmic domain, $\beta_{1 D}$-integrin binds to melusin, a striated muscle-specific protein involved in mechanical signaling, promoting cardiac compensation and preventing failure $[6,10]$. The latter was concluded from experiments in mice after aortic constriction. Melusin-null animals exhibited less hypertrophy and more rapid LV dilation and failure [6], whereas transgenic mice overexpressing melusin showed a prolonged phase of compensated hypertrophy in the absence of fibrosis as compared with wild-type animals [10]. In wild-type mice, aortic constriction led to an overexpression of melusin after $1 \mathrm{wk}$ during compensated hypertrophy, whereas melusin returned to baseline, when LV dilation and impaired contractility ensued after 12 wk [10]. Interestingly, we found a similar temporal expression pattern of melusin after $A V B$, with an upregulation at 3 days $A V B$ and a gradual decline thereafter. It is tempting to speculate that, early after $A V B$, increased melusin expression promotes compensated hypertrophy through mechanisms that remain to be elucidated. This pro-compensatory effect of melusin appeared closely related to the stimulation of hypertrophic growth [6], which could also hold for the AVB model, where melusin overexpression coincides with measures of peak hypertrophic growth (Figure 6A).

In downstream hypertrophic signaling, melusin is involved in the phosphorylation of Akt and GSK3B in response to mechanical load $[6,10]$. We found that the overexpression of melusin coincided with the ratiometric increase of P-Akt/Akt and P-GSK3B/GSK3B during a phase of maximal mechanical load (Figure 1, A and B) and hypertrophic growth (Figure 6).

Phosphorylation inhibits the constitutively active antihypertrophic effect of GSK3 $\beta$ and, thereby, likely stimulates different transcriptional events [14].

$M L P$ is another key Z-disk protein involved in mechanotransduction and -sensing. It interacts with $B_{10}$-integrin via $B$-actinin, is associated with the actin cytoskeleton, and was recently identified as a crucial element of the "cardiac mechanical stretch sensor machinery" consisting of a MLP-titintelethonin protein chain [22]. It has been postulated that a defective chain, as in MLP-null mice and a subset of patients with reduced MLP levels and heart failure [41], leads to dilated cardiomyopathy due to malfunctioning mechanosensing [22]. In dogs with AVB, MLP expression gradually increased to significant levels at chronic AVB, which suggests that the MLP-titin-telethonin chain is likely not defective in this model. This is further supported by the finding of BNP release after AVB, which requires, according to a recent study [22], an intact MLP-titin-telethonin chain as prerequisite for BNP increase during mechanical overload. It has recently been shown that MLP plays an important role in the stimulation of cardiomyocyte hypertrophy via activation of the calcineurin-nuclear factor of activated T-cell pathway $[17,18]$. MLP may act as a scaffold protein to facilitate sarcomere assembly [17] and stimulate further downstream signaling [22]. These findings of a procompensa- 
tory role of MLP in other models of hypertrophy $[17,18,22]$ are in agreement with the high correlation of the temporal expression patterns of MLP and increased LV mass after AVB (Figure 6). We found that, after AVB, MLP expression increased in both the cardiomyocyte cytoplasm and nucleus (Figure 5). These subcellular localizations have been described for MLP and other LIM proteins [21]. Nuclear relocation in conjunction with MLP downregulation has been linked to a "phenoconversion" toward heart failure [12]. However, in our model with compensated hypertrophy, the nuclear translocation is associated with increased MLP levels, suggesting increased transcriptional activity, as also postulated for other LIM proteins [21].

The other Z-disk LIM domain protein that we studied, fhl2, also colocalizes with $\beta_{10}$-integrin, $B$-actinin, and the actin cytoskeleton and is relevant for cardiomyocyte differentiation and myofibrillogenesis [20], as described for MLP. In contrast, fhl2 expression during AVB is transiently reduced around 10 days of AVB. This implicates a different role for fhl 2 in the AVB model, which should be analyzed in more detail in future experiments. Importantly, fhl2 and MLP have a divergent protein structure, which is compatible with a different functional role [21]. In contrast with MLP, fhl2 is regarded as a repressor of hypertrophy [29]. It interacts with ERK2, an element of the MAP kinase signaling pathway. Hypertrophic responses induced by adrenergic stimulation, and mediated via ERK2, are partially antagonized by fhl2, which attenuates transcription [29]. In line with this finding, fhl2-null mice, which exhibit a normal cardiac phenotype, show increased hypertrophic growth after catecholamine infusion, supporting repressor-like activity of fhl2 in the overloaded myocardium [23]. ERK2 interacts with fhl2, but it does not interact with MLP, which underscores the protein-specific action of LIM proteins [29]. The early transient downregulation of fhl2 after AVB coincides with an enhanced adrenergic tone [34] and maximal hypertrophic growth in the first 10 days after atrioventricular block. It could well be that hypertrophy is partly caused by enhanced adrenergic stimulation and mediated via MAP kinase/ERK2. The latter pathway could be incompletely repressed by the fhl 2 reduction at this stage, promoting hypertrophy.

\section{Transitory changes of cytoskeletal elements after AVB}

The depletion of sarcomeres and glycogen accumulation at 10 days AVB was similar but less extensive than reported in chronic hibernating myocardium ( 30\%) [4]. These morphological changes have been interpreted as signs of myocardial dedifferentiation [4]. Interestingly, glycogen accumulation after AVB coincided with the ratiometric increase of P-GSK3 $\beta /$ GSK3 $\beta$, which interacts with glycogen synthase, promoting glycogen synthesis and accumulation [14]. Whether this observation is a purely metabolic phenomenon or a sign of beneficial cardiomyocyte remodeling remains to be elucidated.

The critical changes of the early phase after AVB are further characterized by the transient downregulation of desmin, which is the most important intermediate filament in cardiomyocytes and essential for their structural integrity and function, as recently reviewed [9]. It forms a scaffold that interconnects adjacent myofibrils at the $Z$ disks, serving as a physical link between the sarcolemma, cytoskeleton, and the nuclear envelope $[9,22,30]$. A transient downregulation of desmin 
early during acquired mechanical overload has not been reported in the literature, but it could be a consequence of the abrupt mechanical impact of AVB exhibiting aspects of mild heart failure. In ventricular hypertrophy, desmin expression has been reported to be either unaltered [35] or increased $[19,38]$, but from studies in desmin knockout mice it has become clear that the absence of desmin is associated with cardiac hypertrophy and failure $[9,26]$. Our data suggest that the reduced desmin expression is regulated at the transcriptional level. We could not find a dominant role for calpain as a posttranslational modifier, which has been suggested with respect to the depletion of sarcomeres [8] and desmin disintegration [27], although we have only analyzed protein levels of the regulatory subunit of calpain I and II and not its protease activity, nor other proteases.

Importantly, during chronic AVB the degree of sarcomere depletion, glycogen accumulation, and total desmin content had normalized and desmin was even upregulated in a subset of dogs, especially at the intercalated disk. Since contractile activity has been reported to increase desmin expression by increased gene transcription [39], it could well be that the persistently high levels of ejection strain contribute to the normalization of desmin expression in the chronic phase of AVB.

\section{CONCLUSIONS}

The mechanical load acutely imposed on the ventricular myocardium after AVB causes a transient compromise of cytoskeletal integrity. This is based, at least partly, on transcriptional downregulation. By yet unknown molecular mechanisms, the dog with AVB is able to prevent serious disruption of sarcomeric elements and further downregulation of the important intermediate filament protein desmin. Toward chronic AVB a gradual structural reorganization and a strong drive to functionally compensated hypertrophy are attended by the early upregulation of melusin and MLP, known as procompensatory proteins in other models of ventricular hypertrophy and failure.

\section{Acknowledgments}

The authors thank Monique de Jong, Department of Cardiology; Theo van der Nagel, Department of Cardiothoracic Surgery; Marcel Borgers and Marie-Hélène Lenders, Department of Molecular Cell Biology; Ramon Langen, Department of Respiratory Medicine; and Iwan de Jong, IDEE, Maastricht University, for support and technical assistance. The authors are very grateful to Dr. Mara Brancaccio (melusin), Dr. Pico Caroni (MLP), and Dr. Sabina Kupershmidt (fhl2) for generously providing antibodies.

\section{Grants}

D. W. Donker was financially supported by Medtronic, The Netherlands.

P. G. A. Volders was supported by The Netherlands Organization for Health Research and Development (ZonMW 906-02-068) and the "Stichting Hartsvrienden Rescar", Maastricht, The Netherlands. 


\section{REFERENCES}

1. Arber S, Halder G, Caroni P. Muscle LIM protein, a novel essential regulator of myogenesis, promotes myogenic differentiation. Cell 79: 221-231, 1994.

2. Arts T, Bovendeerd PH, Prinzen FW, Reneman RS. Relation between left ventricular cavity pressure and volume and systolic .ber stress and strain in the wall. Biophys J 59: 93-102, 1991.

3. Asano K, Masuda K, Okumura M, Kadosawa T, Fujinaga T. Plasma atrial and brain natriuretic peptide levels in dogs with congestive heart failure. J Vet Med Sci 61: 523-529, 1999.

4. Ausma J, Thone F, Dispersyn GD, Flameng W, Vanoverschelde JL, Ramaekers FC, Borgers M. Dedifferentiated cardiomyocytes from chronic hibernating myocardium are ischemia-tolerant. Mol Cell Biochem 186: 159-168, 1998.

5. Babbitt CJ, Shai SY, Harpf AE, Pham CG, Ross RS. Modulation of integrins and integrin signaling molecules in the pressure-loaded murine ventricle. Histochem Cell Biol 118: 431-439, 2002.

6. Brancaccio M, Fratta L, Notte A, Hirsch E, Poulet R, Guazzone S, De Acetis M, Vecchione C, Marino G, Altruda F, Silengo L, Tarone G, Lembo G. Melusin, a muscle-specific integrin beta1-interacting protein, is required to prevent cardiac failure in response to chronic pressure overload. Nat Med 9: 68-75, 2003.

7. Brancaccio M, Guazzone S, Menini N, Sibona E, Hirsch E, De Andrea M, Rocchi M, Altruda F, Tarone G, Silengo L. Melusin is a new muscle-specific interactor for beta(1) integrin cytoplasmic domain. J Biol Chem 274: 29282-29288, 1999.

8. Brundel BJ, Ausma J, van Gelder IC, Van der Want JJ, van Gilst WH, Crijns HJ, Henning RH. Activation of proteolysis by calpains and structural changes in human paroxysmal and persistent atrial .brillation. Cardiovasc Res 54: 380-389, 2002.

9. Capetanaki Y. Desmin cytoskeleton in healthy and failing heart. Heart Fail Rev 5: 203-220, 2000.

10. De Acetis M, Notte A, Accornero F, Selvetella G, Brancaccio M, Vecchione C, Sbroggio M, Collino F, Pacchioni B, Lanfranchi G, Aretini A, Ferretti R, Maffei A, Altruda F, Silengo L, Tarone G, Lembo G. Cardiac overexpression of melusin protects from dilated cardiomyopathy due to long-standing pressure overload. Circ Res 96: 1087-1094, 2005.

11. Donker DW, Volders PG, Arts T, Bekkers BC, Hofstra L, Spatjens RL, Beekman JD, Borgers M, Crijns HJ, Vos MA. End-diastolic myofiber stress and ejection strain increase with ventricular volume overload-Serial in-vivo analyses in dogs with complete atrioventricular block. Basic Res Cardiol 100: 372-382, 2005.

12. Ecarnot-Laubriet A, De Luca K, Vandroux D, Moisant M, Bernard C, Assem M, Rochette L, Teyssier JR. Downregulation and nuclear relocation of MLP during the progression of right ventricular hypertrophy induced by chronic pressure overload. J Mol Cell Cardiol 32: 2385-2395, 2000.

13. Ervasti JM. Costameres: the Achilles' heel of Herculean muscle. J Biol Chem 278: 13591-13594, 2003.

14. Hardt SE, Sadoshima J. Negative regulators of cardiac hypertrophy. Cardiovasc Res 63: 500-509, 2004.

15. Hautala N, Tenhunen 0, Szokodi I, Ruskoaho $H$. Direct left ventricular wall stretch activates GATA4 binding in perfused rat heart: involvement of autocrine/paracrine pathways. P.u"gers Arch 443: 362-369, 2002. 
16. He KL, Dickstein M, Sabbah HN, Yi GH, Gu A, Maurer M, Wei CM, Wang J, Burkhoff D. Mechanisms of heart failure with well preserved ejection fraction in dogs following limited coronary microembolization. Cardiovasc Res 64: 72-83, 2004.

17. Heineke J, Kempf T, Kraft T, Hil.ker A, Morawietz H, Scheubel RJ, Caroni P, Lohmann SM, Drexler H, Wollert KC. Downregulation of cytoskeletal muscle LIM protein by nitric oxide: impact on cardiac myocyte hypertrophy. Circulation 107: 1424-1432, 2003.

18. Heineke J, Ruetten $H$, Willenbockel C, Gross SC, Naguib M, Schaefer A, Kempf T, Hil.ker-Kleiner D, Caroni P, Kraft T, Kaiser RA, Molkentin JD, Drexler H, Wollert KC. Attenuation of cardiac remodeling after myocardial infarction by muscle LIM protein-calcineurin signaling at the sarcomeric Z-disc. Proc Natl Acad Sci USA 102: 1655-1660, 2005.

19. Heling A, Zimmermann R, Kostin S, Maeno Y, Hein S, Devaux B, Bauer E, Klovekorn WP, Schlepper M, Schaper W, Schaper J. Increased expression of cytoskeletal, linkage, and extracellular proteins in failing human myocardium. Circ Res 86: 846 -853, 2000.

20. Johannessen M, Moller S, Hansen T, Moens U, Van Ghelue M. The multifunctional roles of the four-anda-half-LIM only protein FHL2. Cell Mol Life Sci 63: 268-284, 2006.

21. Kadrmas JL, Beckerle MC. The LIM domain: from the cytoskeleton to the nucleus. Nat Rev Mol Cell Biol 5: 920-931, 2004.

22. Knoll R, Hoshijima M, Hoffman HM, Person V, Lorenzen-Schmidt I, Bang ML, Hayashi T, Shiga N, Yasukawa H, Schaper W, McKenna W, Yokoyama M, Schork NJ, Omens JH, McCulloch AD, Kimura A, Gregorio CC, Poller W, Schaper J, Schultheiss HP, Chien KR. The cardiac mechanical stretch sensor machinery involves a $\mathrm{Z}$ disc complex that is defective in a subset of human dilated cardiomyopathy. Cell 111: 943-955, 2002.

23. Kong $Y$, Shelton JM, Rothermel B, Li X, Richardson JA, Bassel-Duby R, Williams RS. Cardiac-specific LIM protein FHL2 modi.es the hypertrophic response to beta-adrenergic stimulation. Circulation 103: $2731-2738,2001$.

24. Kupershmidt S, Yang IC, Sutherland M, Wells KS, Yang T, Yang P, Balser JR, Roden DM. Cardiac-enriched LIM domain protein fhl2 is required to generate I(Ks) in a heterologous system. Cardiovasc Res 56 : 93-103, 2002.

25. Maeda K, Tsutamoto T, Wada A, Hisanaga T, Kinoshita M. Plasma brain natriuretic peptide as a biochemical marker of high left ventricular end-diastolic pressure in patients with symptomatic left ventricular dysfunction. Am Heart J 135: 825-832, 1998.

26. Milner DJ, Taffet GE, Wang X, Pham T, Tamura T, Hartley C, Gerdes AM, Capetanaki Y. The absence of desmin leads to cardiomyocyte hypertrophy and cardiac dilation with compromised systolic function. J Mol Cell Cardiol 31: 2063-2076, 1999.

27. Papp Z, van der Velden J, Stienen GJ. Calpain-I induced alterations in the cytoskeletal structure and impaired mechanical properties of single myocytes of rat heart. Cardiovasc Res 45: 981-993, 2000.

28. Pham CG, Harpf AE, Keller RS, Vu HT, Shai SY, Loftus JC, Ross RS. Striated muscle-specific B1D-integrin and FAK are involved in cardiac myocyte hypertrophic response pathway. Am J Physiol Heart Circ Physiol 279: H2916-H2926, 2000.

29. Purcell NH, Darwis D, Bueno OF, Muller JM, Schule R, Molkentin JD. Extracellular signal-regulated kinase 2 interacts with and is negatively regulated by the LIM-only protein FHL2 in cardiomyocytes. Mol Cell Biol 24: 1081-1095, 2004. 
30. Pyle WG, Solaro RJ. At the crossroads of myocardial signaling: the role of Z-discs in intracellular signaling and cardiac function. Circ Res 94: 296-305, 2004.

31. Ramakers C, Vos MA, Doevendans PA, Schoenmakers M, Wu YS, Scicchitano S, lodice A, Thomas GP, Antzelevitch C, Dumaine R. Coordinated down-regulation of KCNQ1 and KCNE1 expression contributes to reduction of $I_{\mathrm{Ks}}$ in canine hypertrophied hearts. Cardiovasc Res 57: 486-496, 2003.

32. Ross RS. Molecular and mechanical synergy: cross-talk between integrins and growth factor receptors. Cardiovasc Res 63: 381-390, 2004.

33. Shai SY, Harpf AE, Babbitt CJ, Jordan MC, Fishbein MC, Chen J, Omura M, Leil TA, Becker KD, Jiang M, Smith DJ, Cherry SR, Loftus JC, Ross RS. Cardiac myocyte-specific excision of the beta1 integrin gene results in myocardial .brosis and cardiac failure. Circ Res 90: 458 -464, 2002.

34. Stengl M, Ramakers C, Donker DW, Nabar A, Rybin AV, Spatjens RL, van der Nagel T, Wodzig WK, Sipido KR, Antoons G, Moorman AF, Vos MA, Volders PG. Temporal patterns of electrical remodeling in canine ventricular hypertrophy: focus on $\mathrm{I}_{\mathrm{Ks}}$ downregulation and blunted beta-adrenergic activation. Cardiovasc Res 72: 90-100, 2006.

35. Tagawa H, Koide M, Sato H, Zile MR, Carabello BA, Cooper GT. Cytoskeletal role in the transition from compensated to decompensated hypertrophy during adult canine left ventricular pressure overloading. Circ Res 82: 751-761, 1998.

36. Van der Flier A, Kuikman I, Baudoin C, van der Neut R, Sonnenberg A. A novel beta 1 integrin isoform produced by alternative splicing: unique expression in cardiac and skeletal muscle. FEBS Lett 369: 340 - 344, 1995.

37. Volders PG, Sipido KR, Vos MA, Kulcsar A, Verduyn SC, Wellens HJ. Cellular basis of biventricular hypertrophy and arrhythmogenesis in dogs with chronic complete atrioventricular block and acquired torsade de pointes. Circulation 98: 1136-1147, 1998.

38. Wang $\mathrm{X}, \mathrm{Li}$ F, Campbell SE, Gerdes AM. Chronic pressure overload cardiac hypertrophy and failure in guinea pigs: Il. Cytoskeletal remodeling. J Mol Cell Cardiol 31: 319-331, 1999.

39. Watson PA, Hannan R, Carl LL, Giger KE. Desmin gene expression in cardiac myocytes is responsive to contractile activity and stretch. Am J Physiol Cell Physiol 270: C1228-C1235, 1996.

40. Yamamoto K, Dang QN, Maeda Y, Huang H, Kelly RA, Lee RT. Regulation of cardiomyocyte mechanotransduction by the cardiac cycle. Circulation 103: 1459-1464, 2001.

41. Zolk O, Caroni P, Bohm M. Decreased expression of the cardiac LIM domain protein MLP in chronic human heart failure. Circulation 101: 2674-2677, 2000. 
Dirk W. Donker, Paul G.A. Volders, Harry J.G.M. Crijns, Theo Arts.

In preparation and in part presented at the 2007 Annual Scientific Sessions of the American Heart Association.

Circulation 2007;116:11622-11622, 2800 Suppl. S. 
Non-invasive quantification of complete left-ventricular pressure-volume and stress-strain loops in the human heart Application of the CircAdapt model 


\begin{abstract}
Quantification of left-ventricular (LV hemodynamics and mechanics in the human heart has important clinical potential, because it can be used to estimate the severity of acute and chronic cardiac decompensation, as well as the primary factors influencing myocardial remodeling. Pressure-volume and stress-strain relations of the LV allow comprehensive assessment of systolic and diastolic LV function and myocardial loading conditions. Non-invasive assessment of LV function is currently based on, but limited to, Doppler echocardiography or magnetic resonance imaging (MRI). LV-pressure recordings are generally lacking because they require invasive diagnostic techniques. We developed a novel approach to simulate LV intracardiac hemodynamics and myofiber mechanics based on the CircAdapt model (Am J Physiol, 2005) applied in humans with different types of cardiac overload.

In 12 patients, 3 with hypertension, 3 with hypertension and mitral/ aortic regurgitation, 2 with mitral regurgitation, and 4 without ventricular overload, we made patient-specific fits of whole circulation hemodynamics using CircAdapt modeling. To this aim, we identified a novel combination of measured 2-dimensional- and Doppler echocardiographic parameters, and arm cuff-measured blood pressure, which served as input into the model.

Peak systolic (range 118-162 $\mathrm{mmHg}$ ) and end-diastolic LV pressures (range 5-18 $\mathrm{mmHg}$ ) agreed within $\pm 10 \%$ and $\pm 16 \%$ (SD), respectively, with the invasively measured pressures during cardiac catheterization in the same patients, showing the reliability of the CircAdapt simulation. With CircAdapt, systolic and diastolic stress and strain imposed on the myocardium could also be obtained. In validation experiments on 7 adult anesthetized dogs, pressure-volume loops generated by invasive and noninvasive methods for the same heart beats were similar.

In conclusion: non-invasive determination of LV hemodynamics and mechanics can be achieved by CircAdapt simulation in patients with different forms of cardiac mechanical overload.
\end{abstract}




\section{INTRODUCTION}

Enduring hemodynamic overload is an important trigger for myocardial remodeling in common cardiovascular diseases like hypertension, valvular heart disease and after myocardial infarction, ultimately leading to heart failure. Many therapeutic regimens are aimed at lowering the mechanical burden on the heart during overload, as it beneficially influences adverse myocardial remodeling. Yet, clinical monitoring of mechanical overload is ill-practiced, and often lacks accuracy and specificity. It usually relies on indirect measures such as serological biomarkers, arm-cuff blood pressure, Doppler echocardiography and in selected cases invasive catheter-based techniques for hemodynamic tailoring of therapy, i.e., Swan-Ganz monitoring.

A thorough assessment of left-ventricular (LV) mechanics requires simultaneous measurement of cavity pressure and volume during the cardiac cycle, which can currently only be obtained by conductance catheters $[4,21]$. Due to the invasiveness of these measurements this is not routinely applied in clinical practice.

Experimental data provide ample evidence that mechanical stimuli act as principal driving forces for myocardial hypertrophy and failure. These insights have stimulated a growing clinical interest to evaluate LV hemodynamics and mechanics as a basis for novel diagnostic and therapeutic strategies and as prognostic indicators [6]. Especially diastolic mechanics are considered relevant for hypertrophic myocardial remodeling $[16,17,25]$ and elevated LV-filling pressures are a unifying diagnostic and prognostic feature of the failing heart [34].

Generally, calculation of myocardial stress and strain in the LV requires data on LV geometry and forces, i.e., intracavitary pressure imposed on the tissue. Since LV pressure cannot be measured non-invasively, a number of Doppler echocardiographic approaches has been proposed, by which LV-filling pressures can be estimated $[5,9,19,23,26,28,31,34,35,39]$. All these studies use empirical relations of indirect hemodynamic and functional parameters to estimate diastolic cardiac function and filling pressures. Yet, non-invasive methods to determine LV pressure dynamically from beginning to end of diastole are currently not available. Moreover, no straightforward, easy-to-use clinical diagnostic modality exists to perform LV pressure-volume and stress-strain assessment at the bedside.

Therefore, we adjusted the CircAdapt computer model of whole circulation dynamics [3] to fit patient-specific non-invasive measurements using 2-dimensional (2D) and Doppler echocardiography combined with arm-cuff blood pressure. This approach allows completely non-invasive estimation of LV pressure-volume and myofiber stress-strain relations throughout the cardiac cycle. CircAdapt simulated LV hemodynamics were compared to invasive assessment during cardiac catheterization in humans and experimental animals. 


\section{METHODS}

\section{CircAdapt model - principle}

The CircAdapt model of the whole circulation is composed of various modules, representing contractile chambers, compliant large blood vessels, valves and peripheral resistances (Figure 1) [3]. The core of the model is a system of differential equations in state variables, i.e., volumes of chambers and blood vessels, transvalvular flows and sarcomere variables. The most relevant equations are summarized in the Appendix. A major built-in feature is its self-structuring capability, i.e., the model itself generates chronic adaptation of the constituent tissues to mechanical and hemodynamic load, e.g., hypertension or mitral regurgitation (Figure 1). Thus, for a given systemic flow and systemic arterial blood pressure, the sizes of all cavities, walls and blood vessels are the result of a time-dependent redistribution of load-simulating chronic adaptation (Figure 1).

The CircAdapt simulation starts by modeling a standard human circulation using the existing model [3]. Next, patient-specific quantitative information on the individual circulation as obtained by 2Dand Doppler echocardiography and arm-cuff blood-pressure measurements is incorporated into the model using a fitting procedure designed to match the CircAdapt simulation to the measurements. The outcome of this simulation renders the complete cardiac hemodynamics and mechanics, the time courses of LV pressure and volume, and myofiber stress and strain of a specific patient.

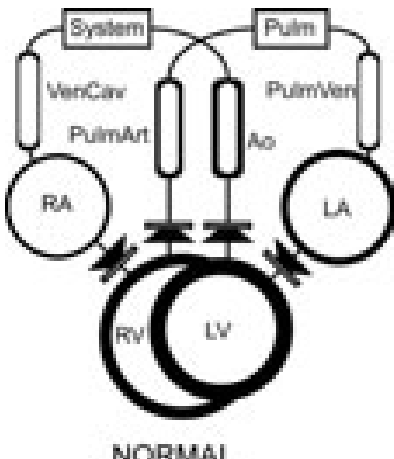

NORMAL

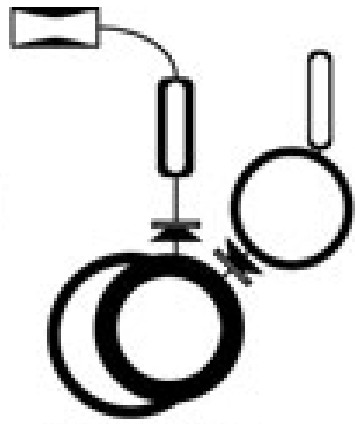

HYPERTENSION

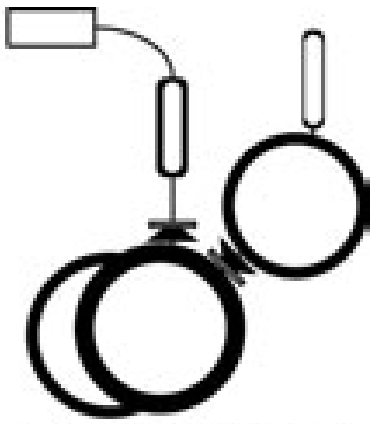

MITRAL REGURGITATION

Figure 1 Left panel, schematic representation of the CircAdapt model. Contractile chambers, large blood vessels, valves and peripheral resistances (System, Pulm) are indicated by circles, rounded boxes, diodes and rectangles, respectively. Symbols LV, RV, LA, and RA represent left and right ventricles and atria, respectively. Symbols Ao, PulmArt, PulmVen and VenCav represent aorta, pulmonary arteries, pulmonary veins and caval vein, respectively. (See Appendix for equations and principles). Mid panel, schematic representation of a CircAdapt-simulated adaptation of the systemic circulation to hypertension. Increasing the peripheral resistance (System) results in concentric hypertrophy of the LV, and increased wall thickness of the Ao. Right panel, schematic representation of a CircAdapt-simulated adaptation of the systemic circulation to mitral regurgitation. Inducing mitral regurgitation results in eccentric hypertrophy of the LV and dilatation of the LA. 


\section{CircAdapt-simulated LV pressure compared to catheterization in humans}

The Medical Ethics Committee of the Academic Hospital Maastricht approved the experiments. All patients gave informed consent to the study.

In 12 patients, 3 with hypertension, 3 with hypertension and mitral/ aortic regurgitation, 2 with mitral regurgitation, and 4 without ventricular overload, cardiac catheterization was performed for clinical diagnostic purposes, i.e., analysis of chest pain or dyspnea, or preoperative work-up prior to valvular surgery. Selection was based on the presence of normally-conducted sinus rhythm, undisturbed regional LV wall motion and the absence of significant coronary artery disease. During catheterization, LV intracavitary- and aortic pressure were measured using standard fluid-filled pig-tail catheters (Biosense Webster). Simultaneously, a standard 12-lead ECG and arm-cuff blood pressure were recorded. Transthoracic echocardiography (Philips Sonos 4500) was performed within 30 minutes after the catheterization in the same supine position accompanied by arm-cuff blood pressure measurement. The relevant patient characteristics are summarized in Table 1.

\section{Echocardiographic measurements and primary analysis}

In every individual, the aortic and mitral pulsed-wave Doppler echocardiographic flow velocities were averaged over 3-5 heart beats by periodically shifting the digitized image along the time

Table 1 Patient characteristics. PO indicates pressure overload, VO indicates volume overload; MR (grade) indicates mitral regurgitation, AR (grade) indicates aortic regurgitation

\begin{tabular}{lllll}
\hline Patient ID & Age & Gender & Type of Overload & Cardiovascular Disease \\
\hline 1500 & 77 & $\mathrm{f}$ & - & - \\
3094 & 53 & $\mathrm{f}$ & - & - \\
5835 & 55 & $\mathrm{~m}$ & - & - \\
9323 & 59 & $\mathrm{~m}$ & - & - \\
& & & & Hypertension \\
5847 & 62 & $\mathrm{~m}$ & $\mathrm{PO}$ & Hypertension \\
6079 & 46 & $\mathrm{f}$ & $\mathrm{PO}$ & Hypertension \\
7059 & 53 & $\mathrm{f}$ & $\mathrm{PO}$ & \\
& & & & Hypertension, MR (mild) \\
0179 & 71 & $\mathrm{f}$ & $\mathrm{PO}, \mathrm{V} 0$ & Hypertension, MR (mild) \\
4996 & 60 & $\mathrm{~m}$ & P0, V0 & Hypertension, MR (mild), AR (mild) \\
5877 & 70 & $\mathrm{f}$ & P0, V0 & \\
& & & & MR (severe) \\
5575 & 55 & $\mathrm{~m}$ & V0 & MR (severe) \\
9388 & 58 & $\mathrm{~m}$ & V0 & \\
\hline
\end{tabular}


axis for the best correlation of the R-wave of the ECG (Figure 2). The time window of averaging, as used for further analysis, started before the R-wave at the beginning of the mitral flow, and extended over one cardiac cycle. The flow velocity and time were calibrated by vertical and horizontal stretching of the image, respectively. Early $\left(v_{e}\right)$ and atrial $\left(v_{a}\right)$ peak mitral velocities and peak aortic velocity $\left(\mathrm{v}_{\mathrm{ao}}\right)$ were determined graphically. The shape, i.e., roundedness, of the aortic flow curve, which is related to kurtosis, was quantified by the ratio of median velocity $\left(v_{\text {med }}\right)$ during ejection over peak velocity $\left(\mathrm{v}_{\mathrm{ao}}\right)$. All times were measured relative to the peak of the R-wave. Timing of aortic flow was defined by valve opening $\left(t_{2}\right)$, peak flow $\left(t_{3}\right)$ and valve closure $\left(t_{4}\right)$. The moment of mitral valve opening $\left(t_{5}\right)$ was defined as the point where the tangent of the rising slope at half peak velocity crossed to zero-flow level. From the point where this tangent crossed the level of maximum velocity $\mathrm{v}_{\mathrm{e}^{\prime}}$ a second line was drawn parallel to the downslope of the early peak at half peak velocity. The latter line crossed the zero velocity axis at time $t_{6}$. The interval $t_{5}-t_{6}$ marks the duration of the early peak. Closure of the mitral valve occurred at moment $t_{1}$.

From 2D echocardiographic parasternal views, short axis internal and external diameters were measured; long-axis length was determined as the distance between the atrial side of the mitralvalve plane and midwall position of the apex at mid ejection. Considering the LV to be a truncated ellipsoid, cavity volume $V_{L V^{\prime}}$ wall volume $V_{w}$ and stroke volume $V_{\text {str }}$ were estimated from the latter dimensions. Systolic and diastolic LV dimensions were determined in the frames just before the beginning and just after the end of ejection, respectively, as described before (17].

\section{CircAdapt simulation - initialization}

Initially, the normal circulation was modeled by CircAdapt simulation as described before [3], using the parameter settings shown in Table 2. General hemodynamics at rest were normal and the diameter of the large bloodvessels and left-sided valves were adjusted until mean flow velocity reached $0.17 \mathrm{~m} / \mathrm{s}$ [8]. Next, a state of exercise was simulated by a threefold systemic flow and a double heart rate. In this state, wall mass and cavity volume of the atria and ventricles were adjusted to satisfy the loading conditions of the sarcomere, i.e., range of myocardial strain, mid value of sarcomere length, and peak passive stress. The wall thickness of the blood vessels was adjusted to set peak wall stress at the level of adaptation. A stable configuration developed after repeated adaptation cycles with rest - exercise - rest - exercise - rest. In the resulting resting state, $\Delta$ fiber strain and maximum passive stress of the sarcomere in the atria and ventricles were stored to define the likely loading levels at chronic normal operation.

\section{CircAdapt simulation - patient-specific modeling}

The present patient-specific simulations focus on the LV, with emphasis on the complete pressurevolume loop including ejection and filling mechanics. All measured parameters (Figure 2) were averaged for the whole group. The resulting set of average values was considered representative 


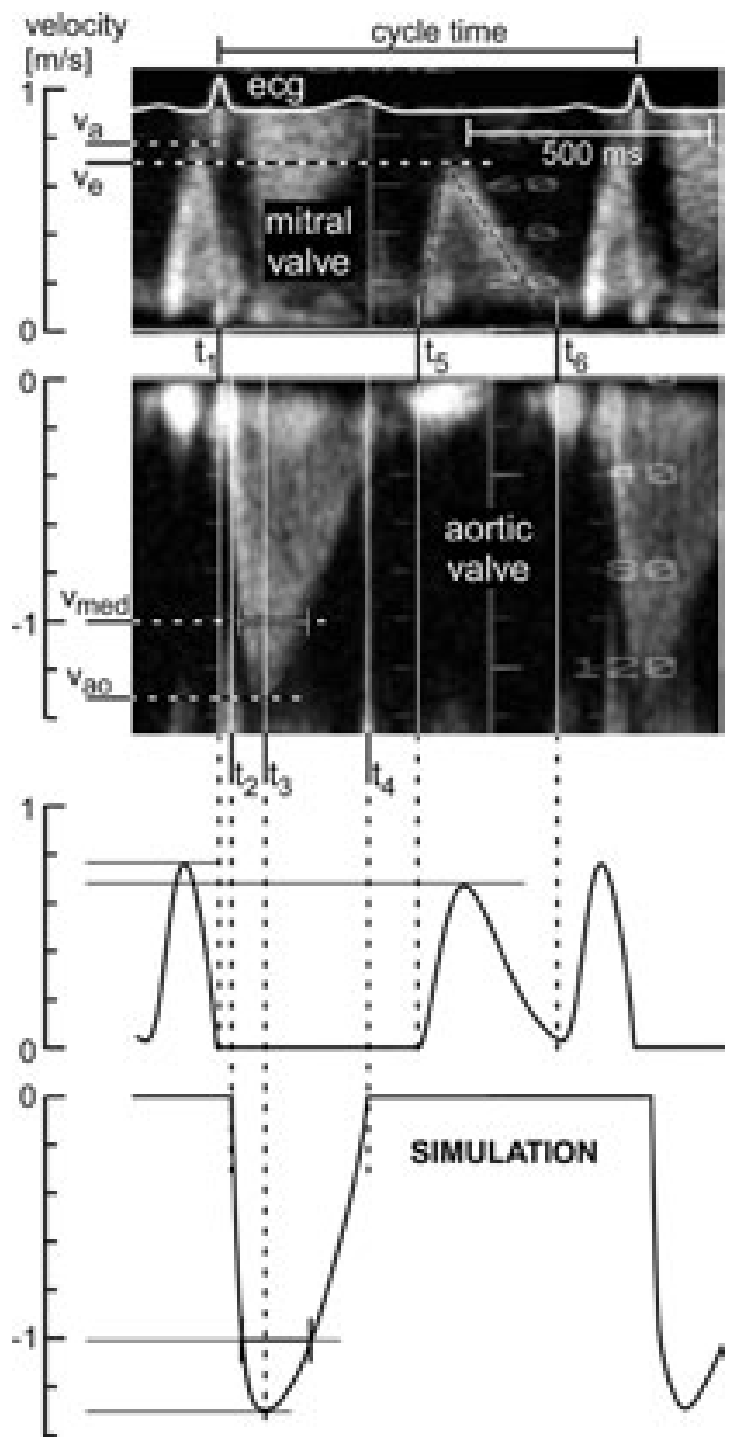

Figure $2 \mid$ Analysis of pulsed-wave Doppler echocardiographic signals. Top panel: From top to bottom, ECG, mitral flow velocity and aortic flow velocity. Images of 3 beats have been overlain. Velocity read ings: $v_{\mathrm{e}}=$ early mitral peak, $\mathrm{v}_{\mathrm{a}}=$ atrial mitral peak $\mathrm{v}_{\text {med }}=$ median ejection velocity, $v_{a 0}=$ peak aortic velocity. Time readings: $t_{1}=$ mitral valve closure, $t_{2}=$ aortic valve opening, $t_{3}=$ peak ejection, $t_{4}=$ aortic valve closure, $t_{5}=$ mitral valve opening, $t_{6}=$ extrapolated ending of early mitral flow peak. Cycle time has been averaged. Bottom graph: Resulting simulation of aortic and mitral flow velocity with CircAdapt in the same format as the top panel. 
Table $2 \mid$ Values as substituted in the CircAdapt model of the normal human circulation

\begin{tabular}{|c|c|c|c|}
\hline & Value & Unit & Remarks \\
\hline \multicolumn{4}{|l|}{ HEMODYNAMICS } \\
\hline Mean systemic flow & 85 & $\mathrm{ml} / \mathrm{s}$ & $=5.1 \mathrm{l} / \mathrm{min}$ \\
\hline Mean aortic pressure & 12.2 & $\mathrm{kPa}$ & $=92 \mathrm{mmHg}$ \\
\hline Cycle time & 850 & $\mathrm{~ms}$ & $\mathrm{HR}=71$ beats $/ \mathrm{min}$ \\
\hline \multicolumn{4}{|l|}{ LARGE BLOOD VESSELS } \\
\hline Mean flow velocity & 0.17 & $\mathrm{~m} / \mathrm{s}$ & [8] \\
\hline Peak wall stress & 500 & $\mathrm{kPa}$ & \\
\hline \multicolumn{4}{|l|}{ SARCOMERE } \\
\hline$\Delta$ Fiber strain in cycle & 0.23 & {$[-]$} & \\
\hline Mean sarcomere length & 1.97 & $\mu \mathrm{m}$ & \\
\hline Maximum passive stress & 2.6 & $\mathrm{kPa}$ & \\
\hline \multicolumn{4}{|l|}{ STATE OF EXERCISE } \\
\hline Mean systemic flow & 255 & $\mathrm{ml} / \mathrm{s}$ & $=15.3 \mathrm{l} / \mathrm{min}$ \\
\hline Cycle time & 425 & $\mathrm{~ms}$ & $H R=142$ beats $/ \mathrm{min}$ \\
\hline
\end{tabular}

for the reference heart beat. Heart rate, mean systemic flow, mean aortic pressure and LV wall volume were substituted in CircAdapt according to the measurements, implying switching off regular chronic LV adaptation to mechanical and hemodynamic load. After simulating a heart cycle, all measured values were determined from the simulation, following the experimental protocol as described in Figure 2. From the differences between simulated and experimentally measured variables, adjustments to the model were calculated (Figure 3). Aortic pulse pressure and stroke volume were used to adjust aortic stiffness. From aortic flow velocity and cardiac output followed the dimension of the aortic valve cross-section. From mean arterial pressure and cardiac output followed the systemic peripheral resistance. Timing of sarcomere contraction followed from timing of aortic-valve opening and closure. LV contractility followed from maintaining LV end-diastolic volume. Timing of events before, during and just after ejection $\left(t_{1}-t_{5}\right)$ was used to adjust timing of ventricular contraction. The dimension of the mitral valve cross-section followed from transmitral flow velocity and cardiac output. The duration of the transmitral early filling wave, i.e., the E-wave, is considered to depend on the resonance frequency, as formed by mitral valve inertia and compliance of the left atrium and LV combined. The E-wave duration was used to adjust the summed stiffness of atrium and ventricle. The ratio of the early filling peak $\left(v_{e}\right)$ and atrial peak $\left(v_{a}\right)$ of transmitral flow velocity (E/A ratio), determines the ratio of ventricular stiffness to atrial stiffness. With increasing 


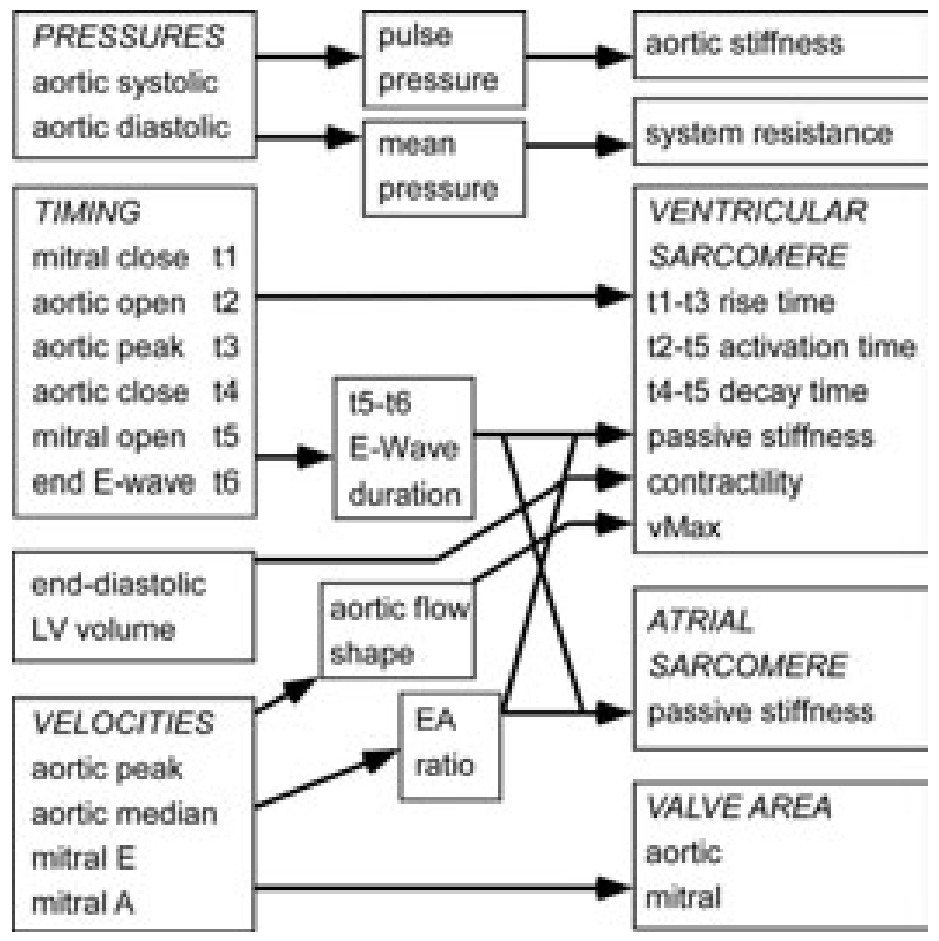

Figure $3 \mid$ Scheme of adjustment of model parameters in patient-specific modeling. Differences between measured and simulated values of pressures, timing, volume and velocities were used to adjust model parameters related to aorta, both ventricles, left atrium and left-sided valves. Time readings: $\mathrm{t} 1=$ mitral valve closure, $\mathrm{t} 2=$ aortic valve opening, $\mathrm{t} 3=$ peak ejection, $\mathrm{t} 4=$ aortic valve closure, $\mathrm{t} 5=$ mitral valve opening, t $6=$ extrapolated ending of early mitral flow peak.

LV stiffness relative to atrial stiffness, early filling is hindered, and atrial contraction is enhanced due to a more prominent volume load of the atrium. An overestimation of end-diastolic LV volume was adjusted by increase of LV contractility. Combination of known diastolic compliances of atrium and ventricle with known volume displacements renders LV diastolic pressure. LV end-diastolic pressure was measured as pressure increase from begin to end diastole.

The ratio of median velocity to peak aortic velocity expresses roundedness of the aortic flow peak, as described above. This parameter was used to adjust velocity of unloaded sarcomere shortening $\mathrm{v}_{\text {sarc }}$. Lowering $\mathrm{v}_{\text {sarc }}$ lowers aortic flow rate at the beginning of ejection, but extends the duration of ejection, resulting in broadening of the flow peak. Except for the LV, all chambers and blood vessels 
regularly adapted size and mass to the varying hemodynamic load, thus mimicking adaptation to the chronic condition.

In the simulation of the reference state, after each cardiac beat, above-described adjustments were carried out. After about 50-100 beats, a steady state was reached. Convergence was not fast and oscillations occurred due to cross-relationships between most variables. The resulting simulation shows blood pressures and flows, and myofiber stresses and strains in all cardiac chambers, blood vessels and over valves as a function of time within a heart beat.

A sensitivity analysis was carried out to investigate the sensitivity of resultant parameters like aortic stiffness, peak passive stress in atrium and ventricle, peak active ventricular stress, velocity of unloaded sarcomere shortening $\mathrm{v}_{\text {sarc }}$ end-diastolic LV pressure, and mean atrial pressure. For the latter analysis, measured times were varied by $10 \mathrm{~ms}$, and the other measured variables were varied by $10 \%$. For each variation, a simulation was performed with the above mentioned fitting procedure.

Finally, feasibility of the method to determine pressure-volume loops non-invasively was tested by carrying out patient-specific simulations, applied to all patients separately. LV intracavitary pressure as measured invasively during catheterization was compared with the LV pressure predicted by the CircAdapt simulation for the same individual.

\section{Experimental validation of CircAdapt-simulated LV pressure by comparison to simultaneous catheterization in adult dogs}

Experiments were conducted in accordance with the 'European Directive for the Protection of Vertebrate Animals Used for Experimental and Other Scientific Purposes (86/609/EU)'. The Committee for Experiments on Animals of Maastricht University approved the experiments.

7 Adult mongrel dogs of either gender and weighing $24 \pm 1 \mathrm{~kg}$ were used. Peri-operative care and anesthesia were performed as described before [16]. All animals were studied under complete anesthesia during normally conducted sinus rhythm. Transthoracic echocardiography and LV intracavitary and aortic pressure measurements were performed simultaneously as described before [17].

\section{Statistical analysis}

Data are presented as mean \pm standard deviation (SD). No statistical comparisons were performed due to the limited number of individuals analyzed in this pilot study. 


\section{RESULTS}

For the 12 patients, group mean values of the measured parameters related to timing of flow events and left-sided hemodynamics are shown in Table 3. All times are indicated relative to the peak of the R-wave in the ECG. The overall variability of the patient data was quite high because of the heterogeneous character of the group. Patient-specific fit of the pulsed-wave Doppler echocardiographic signals was possible in all patients. Its quality is indicated by the simulation results as shown in the bottom panel of Figure 2.

\section{CircAdapt simulation mimics catheterization of the human heart}

CircAdapt-simulated LV-intracavitary pressure curves, which are completely based on noninvasively acquired parameters, yield a high degree of similarity to the invasively-measured data recorded during LV catheterization performed within 30 minutes before the CircAdapt simulation study in the same individual patients (Figure 4). This holds for all individual patients, independent of the presence and type of hemodynamic overload.

The 4 control individuals (Table 1) had LV peak-systolic pressures $\left(\right.$ LVP $\left._{\text {syst }}\right)$ and LV end-diastolic pressures (LVP ${ }_{\text {end-diast }}$ ) during catheterization of $132.0 \pm 5.6$ and $7.7 \pm 2.4 \mathrm{mmHg}$, respectively, which were similar to the CircAdapt simulation yielding $125.8 \pm 10.1$ and $8.8 \pm 1.9 \mathrm{mmHg}$. Patients with pressure overload (PO) or combined PO and volume overload (VO) had LVP syst $_{\text {and LVP }}$ end-diast of $139.0 \pm 14.9$ and $9.9 \pm 2.7 \mathrm{mmHg}$ during catheterization, and $142.3 \pm 16.7$ and $9.6 \pm 4.2 \mathrm{mmHg}$ based on CircAdapt, respectively. Patients with V0 had invasively measured LVP $_{\text {syst }}$ and LVP end-diast $_{\text {of }} 126.0 \pm 9.9$ and $11.7 \pm 0.8 \mathrm{mmHg}$, and simulated values of $130.0 \pm 7.1$ and $14.5 \pm 5.0$, respectively (Figure 5).

$\operatorname{LVP}_{\text {syst }}$ (range $118-162 \mathrm{mmHg}$ ) and $\mathrm{LVP}_{\text {end-diast }}$ (range $5-18 \mathrm{mmHg}$ ) generally agreed within $\pm 10 \%$ and $\pm 16 \%$ with the invasively measured pressures during cardiac catheterization, as compared in the same individual patients, showing the reliability of CircAdapt in a general population of patients with cardiac overload due to cardiovascular disease and in controls (Figure 5). Moreover, besides $L P_{\text {syst }}$ and LVP ${ }_{\text {end-diast' }}$ CircAdapt provides a simulation of the rate of rise and decay of the LV pressure curves (LV dP/dt) (Figure 5).

\section{Sensitivity analysis}

Reference hemodynamics are shown (Figure 6), as obtained by patient-specific fit to the averaged values (Table 3). For these averages no original ultrasound measurements were available of course.

Sensitivity of various parameters is shown for changes in the measured variables (Table 4). The following relationships appeared most prominent. Aortic stiffness $\left(k_{a 0}\right)$ increases more than linear (exponent 1.4 ) with aortic pulse pressure. Peak atrial passive stress $\left(\mathrm{S}_{\text {pasA }}\right)$ is more than proportional (exponent $~ 1.7$ ) with the E/A ratio. Peak passive ventricular stress $\left(\mathrm{S}_{\text {pasv }}\right)$ increases with stroke 
Table $3 \mid$ Summary of measured parameters in 12 patients

\begin{tabular}{|c|c|c|c|}
\hline & & Mean \pm SD & Unit \\
\hline \multicolumn{4}{|l|}{ TIMING ( $\left.\mathrm{t}_{\text {Rwave }}=0 \mathrm{~ms}\right)$} \\
\hline Cycle time & & $945 \pm 121$ & $\mathrm{~ms}$ \\
\hline Opening aortic valve & (t2) & $28 \pm 22$ & $\mathrm{~ms}$ \\
\hline Peak aortic flow & (t3) & $110 \pm 23$ & $\mathrm{~ms}$ \\
\hline Closure aortic valve & (t4) & $335 \pm 26$ & $\mathrm{~ms}$ \\
\hline Opening mitral valve & $(\mathrm{t} 5)$ & $407 \pm 22$ & $\mathrm{~ms}$ \\
\hline End of E-peak & (t6) & $711 \pm 58$ & $\mathrm{~ms}$ \\
\hline A peak mitral flow & & $-75 \pm 41$ & $\mathrm{~ms}$ \\
\hline Closure mitral valve & (t1) & $-9 \pm 32$ & $\mathrm{~ms}$ \\
\hline \multicolumn{4}{|l|}{ VELOCITIES } \\
\hline Peak aortic velocity & & $1.37 \pm 0.28$ & $\mathrm{~m} / \mathrm{s}$ \\
\hline Median aortic velocity & & $0.98 \pm 0.21$ & $\mathrm{~m} / \mathrm{s}$ \\
\hline E-peak mitral velocity & & $0.74 \pm 0.15$ & $\mathrm{~m} / \mathrm{s}$ \\
\hline A-peak mitral velocity & & $0.73 \pm 0.22$ & $\mathrm{~m} / \mathrm{s}$ \\
\hline \multicolumn{4}{|l|}{ PRESSURES } \\
\hline Max. aortic pressure & & $130 \pm 16$ & $\mathrm{mmHg}$ \\
\hline Min. aortic pressure & & $75 \pm 6$ & $\mathrm{mmHg}$ \\
\hline \multicolumn{4}{|l|}{ VOLUMES } \\
\hline Stroke volume & & $68 \pm 18$ & $\mathrm{ml}$ \\
\hline End diastolic volume & & $106 \pm 25$ & $\mathrm{ml}$ \\
\hline LV wall volume & & $153 \pm 45$ & $\mathrm{ml}$ \\
\hline
\end{tabular}

volume and mitral peak velocity. The decrease of the atrial and ventricular stress with increase of LV wall volume can be easily understood, because stress decreases with increasing wall thickness for a given pressure load. The shape of the aortic flow curve, as expressed by the ratio of median to peak aortic flow velocity, decreases with velocity of unloaded sarcomere shortening $v_{\text {sarc }}$. Mean left atrial pressure increases with early peak velocity.

Considering the measurements of timing, the most prominent relations are the following: sarcomere velocity $v_{\text {sarc }}$ highly depends on shape, i.e., if the moment of mitral valve closure is late, aortic valve opening early, and its closure late, the start of the aortic flow curve is steep, and ejection time is long. The stress $S_{\text {pasV }}$ and diastolic pressures $p_{\text {ed }}$ and $p_{L A}$ decrease with the duration of the E-wave $\left(t_{6}-t_{5}\right)$, indicating decrease of stiffness of the compliance-inertia circuit around the mitral valve. Interestingly, this dependency has not been found previously for $\mathrm{S}_{\text {pasA }}$. 


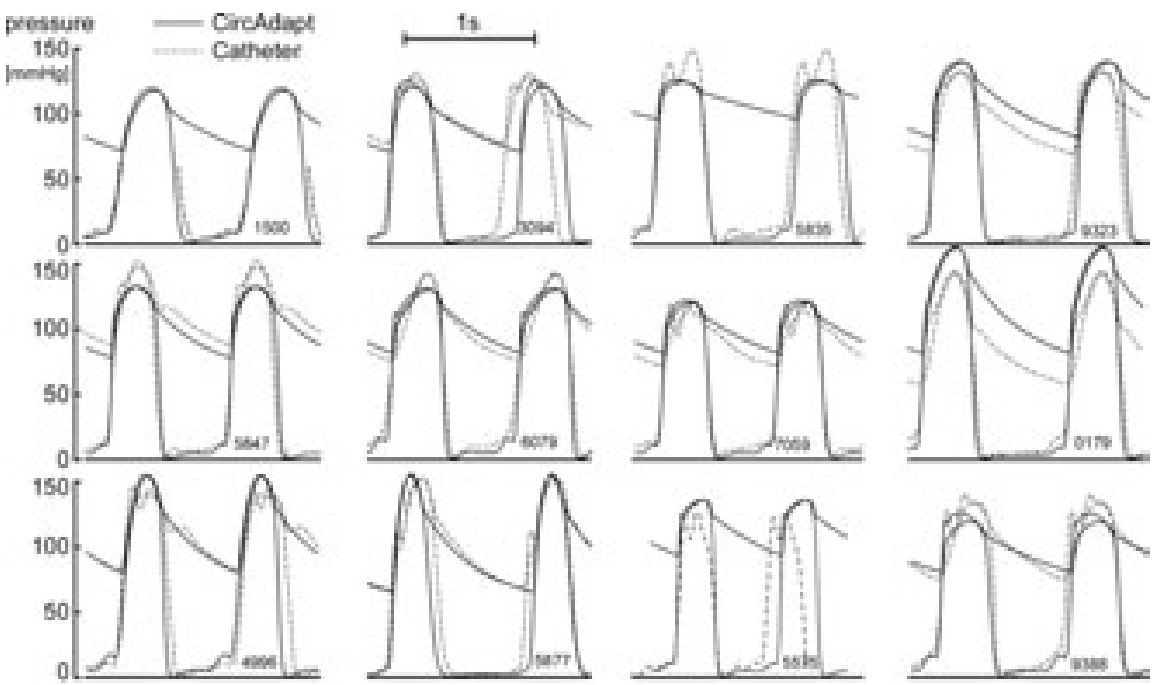

Figure $4 \mid$ Comparison of simulated and invasively measured LV and aortic pressure curves for all individual patients. Individual patient characteristics are summarized in Table 1. Differences in timing between simulated and invasively measured pressures are a result of small differences in heart rate between time-delayed measurements.
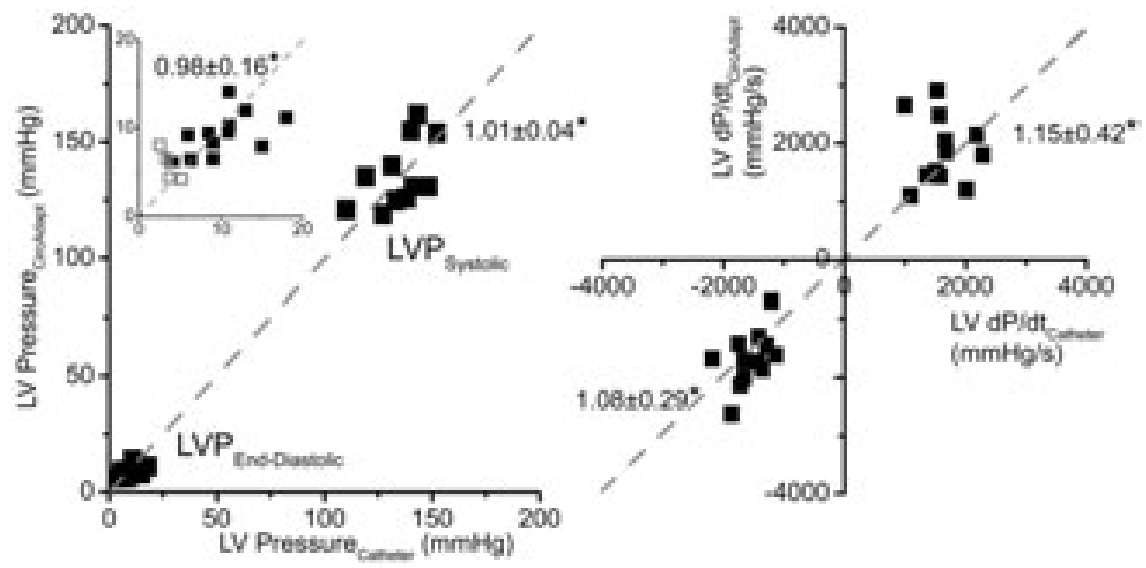

Figure 5 | Patient-specific CircAdapt simulated LV pressures compared to invasive measurements in all individual patients (black rectangles) and dogs (open rectangles, left panel, insert). LVP $P_{\text {end-diast }}$ indicates LV end-diastolic pressure defined as end-diastolic pressure

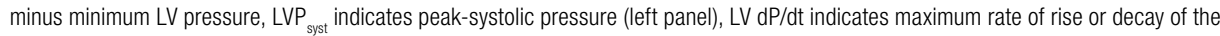
LV pressure curves (right panel), the line of unity is indicated by dashed lines. ${ }^{\star}$ Indicates mean slope of comparison. 

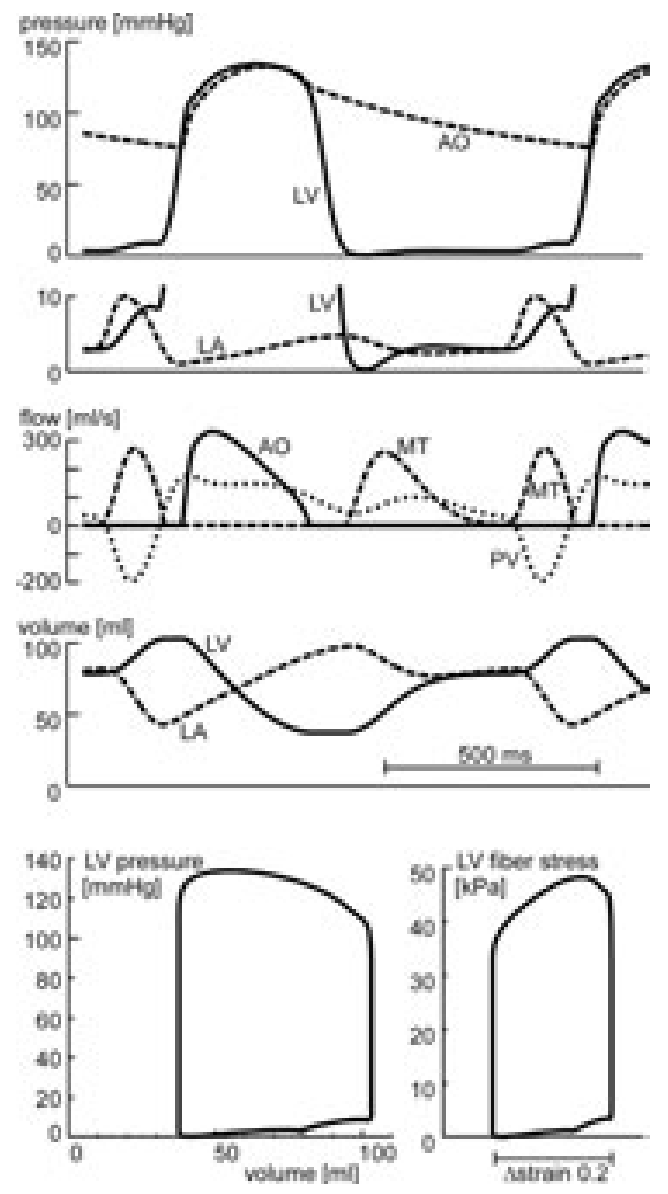

Figure 6 | Hemodynamics by patient-specific modeling to the average values, as presented in Table 3. From top to bottom: Aortic (AO) and LV pressure; (LV) and left atrial (LA) pressure (5x); $A 0$, mitral (MT) and pulmonary venous (PV) flow; LV and LA volume. Bottom left: Pressure - volume loops of LV. Bottom right: Fiber stress - fiber strain loops of LV.

\section{CircAdapt simulation directly compared to simultaneous catheterization in adult dogs}

A direct comparison of invasively measured and CircAdapt-simulated LV pressure curves was performed in adult dogs. In this analysis invasive measurements and data acquisition for the CircAdapt simulation were recorded simultaneously on a beat-to-beat basis and were not time-delayed as in the human study. This analysis revealed a reasonable overall accuracy of the CircAdapt simulation comparing complete curves covering the whole cardiac cycle. Group mean measured $\mathrm{LVP}_{\text {end-diast }}$ was $3.8 \pm 1.0 \mathrm{mmHg}$, which is the most critical LV pressure parameter within the cardiac cycle, and was estimated by CircAdapt as being $5.9 \pm 1.6 \mathrm{mmHg}$ (Figure 5). 
Table 4 Sensitivity analysis, the numbers indicate relative sensitivity $\mathrm{d} \ln \left(\mathrm{Par}_{{ }_{\text {out }}}\right) / \mathrm{dt}$ in $\left[\mathrm{s}^{-1}\right]$ for timing parameters, and $\mathrm{d} \ln \left(\mathrm{Par}_{\text {out }}\right) / \mathrm{d} \ln \left(\mathrm{Par}_{\text {in }}\right)$ for the other parameters

\begin{tabular}{|c|c|c|c|c|c|c|c|}
\hline $\mathrm{Par}_{\text {in }} \downarrow \mathrm{Par}_{\text {out }} \rightarrow$ & $\mathrm{k}_{\mathrm{ao}}$ & $\mathrm{S}_{\mathrm{pasV}}$ & $\mathrm{S}_{\text {pasA }}$ & $\mathrm{S}_{\text {actV }}$ & $v_{\text {sarc }}$ & $p_{e d}$ & $p_{L A}$ \\
\hline & {$[-]$} & $\mathrm{kPa}$ & $\mathrm{kPa}$ & $\mathrm{kPa}$ & $\mu \mathrm{m} / \mathrm{s}$ & $\mathrm{kPa}$ & $\mathrm{kPa}$ \\
\hline Reference value & 8.32 & 2.6 & 14.9 & 51.0 & 3.82 & 1.12 & 0.51 \\
\hline \multicolumn{8}{|l|}{ TIMING $\left[\mathrm{s}^{-1}\right]$} \\
\hline Cycle time & -0.7 & 0.4 & -0.1 & 0.3 & -0.1 & 0.1 & -0.1 \\
\hline Opening aortic valve (t2) & -0.9 & 3.8 & 1.2 & 1.3 & 13.3 & -2.1 & -0.3 \\
\hline Peak aortic flow (t3) & -0.2 & -0.9 & 0.7 & -2.1 & 1.9 & -1.1 & -0.6 \\
\hline Closure aortic valve (t4) & 1.6 & -3.2 & -1.5 & -2.1 & -6.7 & 0.8 & -0.7 \\
\hline Opening mitral valve (t5) & 0.0 & 7.8 & -0.1 & 1.6 & -0.5 & 6.3 & 4.6 \\
\hline End of E-peak (t6) & 0.2 & -6.4 & 1.0 & -0.3 & 0.5 & -5.9 & -3.5 \\
\hline Closure mitral valve (t1) & 0.1 & -1.4 & -1.1 & 1.3 & -8.3 & 1.8 & 0.5 \\
\hline \multicolumn{8}{|l|}{ VELOCITIES } \\
\hline Peak aortic velocity & -0.1 & 0.1 & 0.0 & -0.4 & 1.7 & -0.2 & 0.2 \\
\hline Median aortic velocity & 0.1 & 0.1 & -0.3 & 0.7 & -1.9 & 0.6 & 0.3 \\
\hline E-peak mitral velocity & 0.0 & 0.5 & 1.7 & 0.0 & 0.0 & 0.2 & 1.2 \\
\hline A-peak mitral velocity & 0.0 & 1.1 & -1.7 & 0.0 & -0.1 & 1.2 & 0.7 \\
\hline \multicolumn{8}{|l|}{ PRESSURES } \\
\hline Max. aortic pressure & 1.4 & -0.1 & 0.2 & 0.8 & 0.0 & 0.0 & 0.1 \\
\hline Min. aortic pressure & -1.4 & 0.0 & -0.3 & 0.1 & -0.2 & 0.3 & 0.3 \\
\hline \multicolumn{8}{|l|}{ VOLUMES } \\
\hline Stroke volume & 0.0 & 1.6 & -0.9 & 0.3 & 0.3 & 1.4 & 0.7 \\
\hline End diastolic volume & 0.0 & 0.3 & 0.8 & 0.6 & 0.4 & -0.6 & 0.0 \\
\hline LV wall volume & 0.0 & -1.5 & -0.2 & -1.0 & -0.9 & -0.1 & 0.1 \\
\hline
\end{tabular}

$\mathrm{k}_{\mathrm{a} 0}=$ aortic stiffness, $\mathrm{S}_{\text {pasv }}=$ passive LV stress, $\mathrm{S}_{\text {pasA }}=$ passive LA stress, $\mathrm{S}_{\text {actv }}=$ active LV stress, $\mathrm{v}_{\text {sarc }}=$ sarcomere velocity, $\mathrm{p}_{\mathrm{ed}}=$ end-diastolic LV pressure, $\mathrm{p}_{\mathrm{LA}}=$ mean left atrial pressure. 


\section{DISCUSSION}

The CircAdapt model allows the simulation of LV hemodynamics and dimensions in individual patients in an entirely non-invasive way. On the basis of parameters derived from transthoracic echocardiography and arm-cuff-measured blood pressure we demonstrate reasonable agreement between simulated and invasively measured data in human patients with different forms of cardiac mechanical overload.

\section{CircAdapt provides non-invasive patient-specific simulation of LV-pressure curves}

As a unique result, the CircAdapt computer simulation generates LV intracavitary pressure curves during the whole cardiac cycle. These pressure curves can be simulated for individual patients by completely non-invasive means.

Only a few studies have reported comparable approaches of echocardiographic 'pressure imaging'. It has been shown that $\mathrm{LV}$ intraventricular pressure gradients can be estimated non-invasively during diastole from digitized echocardiographic color M-mode maps of transmitral flow [10-12,23,24]. Yet, in these studies only a part of the cardiac cycle is addressed, whereas the CircAdapt simulation provides dynamic pressure data with a high temporal resolution throughout the cardiac cycle.

In addition, a number of echocardiographic approaches have been presented to estimate LV-filling pressures or related parameters, i.e., LV end-diastolic pressures [31] or mean capillary wedge pressures [34]. These approaches are based on parameters derived from transmitral and pulmonary vein Doppler echocardiographic flow pattern, often combined with tissue Doppler echocardiographic measurements of mitral annular or myocardial tissue velocities [1,5,19,20,26,28,30-33,36,37,45]. In these studies, elevated LV filling pressures could be predicted with reasonable accuracy. Yet, absolute intracavitary pressure values, as in CircAdapt, could not directly be derived from the echocardiographic data.

\section{CircAdapt provides non-invasive patient-specific assessment of LV pressure-volume and stress-strain loops}

In addition to the information on LV intracavitary pressure, CircAdapt also provides data on complete pressure-volume relationships throughout the cardiac cycle, which have not been generated non-invasively before. Only a few studies have addressed this issue by invasive means using pressure or conductance catheters, echocardiography or ultrasonic crystal or using ex vivo techniques, describing diastolic [27] or complete $[43,44]$ pressure-volume relations.

The CircAdapt model does not only provide diastolic pressure-volume information but also data on the systolic phase covering the whole cardiac cycle. This allows e.g., the assessment of parameters as the slope of the end-systolic and end-diastolic pressure-volume relation, which have been considered as measures of contractility and diastolic function and properties by others before 
$[7,41,42]$. In this way CircAdapt may help to improve the characterization of systolic and diastolic cardiac function.

Moreover, the CircAdapt model also provides a comprehensive quantification of LV myocardial tissue mechanics, as evident from the myofiber stress-strain loops in Figure 6. As an example of the usefulness of LV mechanical assessment in the clinical setting, we studied a patient presenting with congestive heart failure due to severe global ischemia as a consequence of multivessel coronary artery disease before and after treatment (Figure 7). Comparable approaches, providing noninvasive comprehensive assessment of patient-specific LV hemodynamics and mechanics in a serial manner, have to the best of our knowledge not been reported before.

\section{A few echocardiographic and arm cuff blood pressure parameters are sufficient to simulate whole circulation dynamics}

A novel combination of 2D and Doppler echocardiographic parameters of transmitral LV inflow, aortic LV outflow is sufficient to simulate whole circulation hemodynamics using the CircAdapt model.

Transmitral flow could be simulated by identifying just a few key parameters from the dynamic Doppler flow profiles. Mitral inflow velocities have been extensively described to reflect LV filling pressures. The early diastolic flow is largely determined by myocardial relaxation and left atrial pressure [34]. The mitral inflow velocity pattern changes characteristically with decreasing filling pressure, i.e., the early diastolic velocity (E) decreases and the deceleration time (DT) of E lengthens. As filling pressure increases, $E$ and the E/A-ratio $(>1)$ increase and the deceleration time shortens [34]. Moreover, it is well known that not only flow velocities but also timing and duration of flow provides valuable information in this regard $[38,40]$. The CircAdapt model accounts for the complexity of mutual dependencies of LV in- and outflow and dimensions by addressing a broad range of relevant parameters, allowing feed-back and cross-relation adjustments in a closed-loop model system. We made a step forward in accuracy by applying a new strategy of non-invasive patient-specific modeling. This improved accuracy compared to conventional estimates is largely achieved by the integration of patient-specific measurements in a simulated complete circulation, which has gone through a process of physiologic adaptation.

\section{Limitations}

The LV intracavitary and aortic pressures measured invasively during catheterization could not be recorded simultaneously with the echocardiogram. In order to assure comparability, all echocardiograms were performed within 30 minutes after the catheterization with the patient lying in an identical supine position. The additionally performed experimental study in adult dogs, allowing a simultaneous comparison of computer simulation and LV catheterization, supports the accuracy of the CircAdapt model found in the series of human patients by providing simulated complete LV 

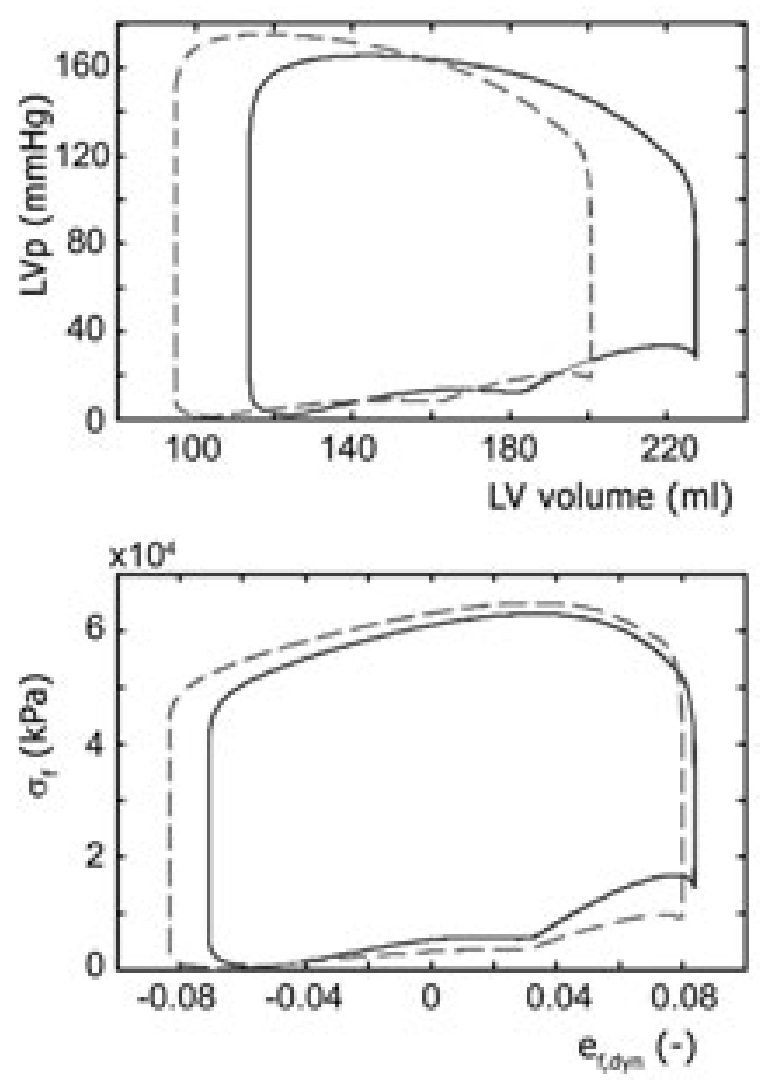

Figure $7 \mid$ CircAdapt simulation of pressure-volume (top panel) and stress-strain (bottom panel) loops of an individual patient presenting with congestive heart failure due to global ischemia as a consequence of multivessel coronary artery disease before (solid line) and after initiation of medical treatment (dashed line).

pressure curves and LV end-diastolic pressure values which are in reasonable agreement with the invasively measured values.

Arm-cuff blood pressure was not significantly different between the catheterization and the echocardiographic recordings. Yet, differences between invasively measured and simulated intracavitary pressures might be explained by methodological differences of blood pressure measurements between catheter-based invasive measurements, automatic cuff measurements and the conventional auscultatory method, as reported in the literature $[18,22,29]$. 
Patient selection was based on normally conducted sinus rhythm, undisturbed regional LV wall motion and the absence of significant coronary artery disease. For a broader clinical implementation, differences in regional wall motion should be incorporated as well as rhythm and conduction disturbances. These issues deserve further improvement of the CircAdapt model to allow a broad implementation in clinical practice.

\section{CONCLUSIONS}

Non-invasive determination of LV hemodynamics and mechanics can be achieved by CircAdapt simulation in patients with different forms of cardiac mechanical overload. Based on a list of timings, standard echocardiographic measures, Doppler flow velocities and arm cuff blood pressure values, a stable, well-defined, and completely non-invasive patient-specific fit can be obtained at the bedside. In addition complete LV pressure tracings, pressure-volume and stress-strain loops can be generated, allowing comprehensive systolic and also diastolic assessment of cardiac function including quantification of end-diastolic pressure and function, considered as important triggers for cardiac remodeling.

\section{Perspective}

The CircAdapt simulation of cardiac hemodynamics and mechanics can be transferred into clinical practice with relative ease and offers the perspective of an easy-to-use bedside hemodynamic and mechanical assessment during various stages of heart disease. In conjunction with serum biomarkers of overload-induced remodeling, favorable and unfavorable patterns of cardiac mechanical overload can be identified in individual patients over time, as shown for other noninvasive approaches [13-15]. This strategy might aid to improve clinical decision making, prognostic judgments, risk-stratification, tailoring of therapy and proper timing of interventions.

The CircAdapt model seems suitable for sequential patient-specific simulation of cardiac overload in an entirely non-invasive way. This will allow assessment of the individual load history over months and years in patients with cardiac disease inducing mechanical overload.

\section{Acknowledgements}

D.W. Donker was financially supported by Medtronic, Netherlands. P.G.A. Volders was supported by the 'Stichting Hartsvrienden Rescar', Maastricht, Netherlands. The authors wish to thank Roel Spätjens, Monique de Jong, Johannes Waltenberger, Marc Winkens, Leo Veenstra, Lex Ruiters, Department of Cardiology, Theo van der Nagel, Department of Cardiothoracic Surgery for their contribution to the study. 


\section{APPENDIX}

\section{Principles of the CircAdapt model}

The CircAdapt model of the whole circulation [3] is designed to simulate beat to beat hemodynamics in the four-chamber heart containing valves and the large bloodvessels. Chambers, valves and vessels are represented by modules, based on temporal differential equations in state variables. By connecting the modules a network forms, representing the whole circulation. The core of the model is a system of differential equations, to be solved by numerical integration. Chambers and blood vessels are cavities with a compliant wall, which gets its stiffness from fibers in the constituting tissue. Using cavity volume $\mathrm{V}_{\text {cav }}$ as a state variable, fiber extension $\mathrm{I} / \mathrm{I}_{\text {ref }}$ is calculated by the one-fiber model [2]:

$$
\frac{I}{I_{\text {ref }}}=\left(1+3 \frac{V_{\text {cav }}}{V_{\text {wall }}}\right)^{1 / 3}
$$

Using a model of fiber mechanics, fiber stress $\left(\sigma_{f}\right)$ is calculated as a function of fiber extension. For fiber stress in chamber walls a model of sarcomere dynamics with two state variables has been used. Fiber stress in the vessel wall is modeled by passive elastic behavior only. Using the one-fiber model, cavity pressure $p_{\text {cav }}$ is calculated from this stress. Thus, it is used:

$$
p_{\text {cav }}=\frac{\sigma_{f}\left(I / I_{\text {ref }}\right)}{1+3 V_{\text {cav }} / V_{\text {wall }}}
$$

Cavities and vessels may be connected by valves or resistances. In a valve, flow $q$ is a state variable. With positive pressure drop $\Delta p$ or forward flow, the valve opens. The time derivative of flow in an open valve is determined by pressure drop over the valve and pressure loss due to the Bernoulli effect:

$$
\frac{\rho}{\sqrt{A_{\text {open }}}} \frac{d q}{d t}=\Delta p-\frac{q^{2}}{2 a_{\text {open }}^{2}}
$$

Parameter $A_{\text {open }}$ represents the cross-section of the open valve. A major feature of the CircAdapt model is that size of cavities and walls adapts so that mechanical load of the constituting tissues is normalized. For instance when mean systemic pressure is elevated, the LV will show concentric hypertrophy. This self-adapting property causes the model to simulate circulation hemodynamics very realistically. The model is programmed in $\mathrm{MATLAB}^{\circledR}$ (www.mathworks.com). Simulation time for one cardiac beat is about 2 seconds on a regular PC. 


\section{REFERENCES}

1. Appleton CP, Galloway JM, Gonzalez MS, Gaballa M, and Basnight MA. Estimation of left ventricular filling pressures using two-dimensional and Doppler echocardiography in adult patients with cardiac disease. Additional value of analyzing left atrial size, left atrial ejection fraction and the difference in duration of pulmonary venous and mitral flow velocity at atrial contraction. J Am Coll Cardiol 22: 19721982, 1993.

2. Arts T, Bovendeerd PH, Prinzen FW, and Reneman RS. Relation between left ventricular cavity pressure and volume and systolic fiber stress and strain in the wall. Biophys J 59: 93-102, 1991.

3. Arts T, Delhaas T, Bovendeerd P, Verbeek X, and Prinzen FW. Adaptation to mechanical load determines shape and properties of heart and circulation: the CircAdapt model. Am J Physiol Heart Circ Physiol 288: H1943-1954, 2005.

4. Baan J, van der Velde ET, de Bruin HG, Smeenk GJ, Koops J, van Dijk AD, Temmerman D, Senden J, and Buis B. Continuous measurement of left ventricular volume in animals and humans by conductance catheter. Circulation 70: 812-823, 1984.

5. Borekci A, Karakelleoglu S, Erol MK, Bozkurt E, and Yilmaz M. The usefulness of tissue doppler imaging in the determination of left ventricular diastolic filling pressure. Comparison with other techniques. Acta Cardiol 61: 129-135, 2006.

6. Burkhoff D, Mirsky I, and Suga H. Assessment of systolic and diastolic ventricular properties via pressurevolume analysis: a guide for clinical, translational, and basic researchers. Am J Physiol Heart Circ Physiol 289: H501-512, 2005.

7. Claessens TE, Rietzschel ER, De Buyzere ML, De Bacquer D, De Backer G, Gillebert TC, Verdonck PR, and Segers $P$. Noninvasive assessment of left ventricular and myocardial contractility in middle-aged men and women: disparate evolution above the age of 50? Am J Physiol Heart Circ Physiol 292: H856-865 2007.

8. Dawson TH. Similitude in the cardiovascular system of mammals. J Exp Biol 204: 395-407, 2001.

9. De Boeck BW, Oh JK, Vandervoort PM, Vierendeels JA, van der Aa RP, and Cramer MJ. Colour M-mode velocity propagation: a glance at intra-ventricular pressure gradients and early diastolic ventricular performance. Eur J Heart Fail 7: 19-28, 2005.

10. De Mey S, De Sutter J, Vandervoort P, De Buyzere M, and Verdonck P. Assesment of LV diastolic filling using color M-mode Doppler echocardiography: validation in new hydoraulic model. Biomech Model Mechanobiol 2: 127-138, 2004.

11. De Mey S, De Sutter J, Vierendeels J, and Verdonck P. Diastolic filling and pressure imaging: taking advantage of the information in a colour M-mode Doppler image. Eur J Echocardiogr 2: 219-233, 2001.

12. De Mey S, Thomas JD, Greenberg NL, Vandervoort PM, and Verdonck PR. Assessment of the time constant of relaxation: insights from simulations and hemodynamic measurements. Am J Physiol Heart Circ Physiol 280: H2936-2943, 2001.

13. Diwan A, McCulloch M, Lawrie GM, Reardon MJ, and Nagueh SF. Doppler estimation of left ventricular filling pressures in patients with mitral valve disease. Circulation 111: 3281-3289, 2005. 
14. Dokainish H, Zoghbi WA, Lakkis NM, Al-Bakshy F, Dhir M, Quinones MA, and Nagueh SF. Optimal noninvasive assessment of left ventricular filling pressures: a comparison of tissue Doppler echocardiography and B-type natriuretic peptide in patients with pulmonary artery catheters. Circulation 109: 2432-2439, 2004.

15. Dokainish H, Zoghbi WA, Lakkis NM, Quinones MA, and Nagueh SF. Comparative accuracy of B-type natriuretic peptide and tissue Doppler echocardiography in the diagnosis of congestive heart failure. $A m$ J Cardio/ 93: 1130-1135, 2004.

16. Donker DW, Maessen JG, Verheyen F, Ramaekers FC, Spatjens RL, Kuijpers H, Ramakers C, Schiffers PM, Vos MA, Crijns HJ, and Volders PG. Impact of acute and enduring volume overload on mechanotransduction and cytoskeletal integrity of canine left ventricular myocardium. Am J Physiol Heart Circ Physiol 292: H2324-2332, 2007.

17. Donker DW, Volders PG, Arts T, Bekkers BC, Hofstra L, Spatjens RL, Beekman JD, Borgers M, Crijns HJ, and Vos MA. End-diastolic myofiber stress and ejection strain increase with ventricular volume overload Serial in-vivo analyses in dogs with complete atrioventricular block. Basic Res Cardiol 100: 372-382, 2005.

18. Finnie KJ, Watts DG, and Armstrong PW. Biases in the measurement of arterial pressure. Crit Care Med 12: $965-968,1984$.

19. Garcia MJ, Ares MA, Asher C, Rodriguez L, Vandervoort P, and Thomas JD. An index of early left ventricular filling that combined with pulsed Doppler peak E velocity may estimate capillary wedge pressure. $J \mathrm{Am}$ Coll Cardiol 29: 448-454, 1997.

20. Geske JB, Sorajja P, Nishimura RA, and Ommen SR. Evaluation of left ventricular filling pressures by Doppler echocardiography in patients with hypertrophic cardiomyopathy: correlation with direct left atrial pressure measurement at cardiac catheterization. Circulation 116: 2702-2708, 2007.

21. Glantz SA, Boltwood CM, Jr., Appleyard RF, Applegate RJ, Cheng CP, and Little WC. Volume conductance catheter. Circulation 81: 2028, 1990.

22. Gravlee GP and Brockschmidt JK. Accuracy of four indirect methods of blood pressure measurement, with hemodynamic correlations. J Clin Monit 6: 284-298, 1990.

23. Greenberg NL, Vandervoort PM, Firstenberg MS, Garcia MJ, and Thomas JD. Estimation of diastolic intraventricular pressure gradients by Doppler M-mode echocardiography. Am J Physiol Heart Circ Physiol 280: H2507-2515, 2001.

24. Greenberg NL, Vandervoort PM, and Thomas JD. Instantaneous diastolic transmitral pressure differences from color Doppler M mode echocardiography. Am J Physiol 271: H1267-1276, 1996.

25. Holmes JW. Candidate mechanical stimuli for hypertrophy during volume overload. J Appl Physiol 97: 1453-1460, 2004.

26. Hurrell DG, Nishimura RA, Ilstrup DM, and Appleton CP. Utility of preload alteration in assessment of left ventricular filling pressure by Doppler echocardiography: a simultaneous catheterization and Doppler echocardiographic study. J Am Coll Cardiol 30: 459-467, 1997.

27. Klotz S, Hay I, Dickstein ML, Yi GH, Wang J, Maurer MS, Kass DA, and Burkhoff D. Single-beat estimation of end-diastolic pressure-volume relationship: a novel method with potential for noninvasive application. Am J Physiol Heart Circ Physiol 291: H403-412, 2006. 
28. Kucher N, Schwerzmann M, Lipp E, Eyer D, Meier B, and Seiler C. Validation of six noninvasive Doppler methods for the assessment of left ventricular filling pressure. Echocardiography 19: 645-653, 2002.

29. Lehmann KG, Gelman JA, Weber MA, and Lafrades A. Comparative accuracy of three automated techniques in the noninvasive estimation of central blood pressure in men. Am J Cardiol 81: 1004-1012, 1998.

30. Nagueh SF, Lakkis NM, Middleton KJ, Spencer WH, 3rd, Zoghbi WA, and Quinones MA. Doppler estimation of left ventricular filling pressures in patients with hypertrophic cardiomyopathy. Circulation 99: 254-261, 1999.

31. Nagueh SF, Middleton KJ, Kopelen HA, Zoghbi WA, and Quinones MA. Doppler tissue imaging: a noninvasive technique for evaluation of left ventricular relaxation and estimation of filling pressures. J Am Coll Cardiol 30: 1527-1533, 1997.

32. Nagueh SF, Sun H, Kopelen HA, Middleton KJ, and Khoury DS. Hemodynamic determinants of the mitral annulus diastolic velocities by tissue Doppler. J Am Coll Cardiol 37: 278-285, 2001.

33. Nishimura RA, Appleton CP, Redfield MM, Ilstrup DM, Holmes DR, Jr., and Tajik AJ. Noninvasive doppler echocardiographic evaluation of left ventricular filling pressures in patients with cardiomyopathies: a simultaneous Doppler echocardiographic and cardiac catheterization study. J Am Coll Cardiol 28: 12261233, 1996.

34. Oh JK. Echocardiography as a noninvasive Swan-Ganz catheter. Circulation 111: 3192-3194, 2005

35. Oh JK, Appleton CP, Hatle LK, Nishimura RA, Seward JB, and Tajik AJ. The noninvasive assessment of left ventricular diastolic function with two-dimensional and Doppler echocardiography. J Am Soc Echocardiogr 10: 246-270, 1997.

36. Ommen SR, Nishimura RA, Appleton CP, Miller FA, Oh JK, Redfield MM, and Tajik AJ. Clinical utility of Doppler echocardiography and tissue Doppler imaging in the estimation of left ventricular filling pressures: A comparative simultaneous Doppler-catheterization study. Circulation 102: 1788-1794, 2000.

37. Poerner TC, Goebel B, Kralev S, Kaden JJ, Suselbeck T, Haase KK, Borggrefe M, and Haghi D. Impact of mitral e/a ratio on the accuracy of different echocardiographic indices to estimate left ventricular end-diastolic pressure. Ultrasound Med Biol 33: 699-707, 2007.

38. Rivas-Gotz C, Khoury DS, Manolios M, Rao L, Kopelen HA, and Nagueh SF. Time interval between onset of mitral inflow and onset of early diastolic velocity by tissue Doppler: a novel index of left ventricular relaxation: experimental studies and clinical application. J Am Coll Cardiol 42: 1463-1470, 2003.

39. Rivas-Gotz C, Manolios M, Thohan V, and Nagueh SF. Impact of left ventricular ejection fraction on estimation of left ventricular filling pressures using tissue Doppler and flow propagation velocity. Am J Cardiol 91: 780-784, 2003.

40. Rossvoll 0 and Hatle LK. Pulmonary venous flow velocities recorded by transthoracic Doppler ultrasound: relation to left ventricular diastolic pressures. J Am Coll Cardiol 21: 1687-1696, 1993.

41. Senzaki $\mathrm{H}$, Chen $\mathrm{CH}$, and Kass DA. Single-beat estimation of end-systolic pressure-volume relation in humans. A new method with the potential for noninvasive application. Circulation 94: 2497-2506, 1996.

42. Takeuchi M, Igarashi Y, Tomimoto S, Odake M, Hayashi T, Tsukamoto T, Hata K, Takaoka H, and Fukuzaki $\mathrm{H}$. Single-beat estimation of the slope of the end-systolic pressure-volume relation in the human left ventricle. Circulation 83: 202-212, 1991. 
43. Urheim S, Bjornerheim R, Endresen K, Vatne K, Rabben SI, Sorhus V, and Smiseth OA. Quantification of left ventricular diastolic pressure-volume relations during routine cardiac catheterization by twodimensional digital echo quantification and left ventricular micromanometer. J Am Soc Echocardiogr 15: 225-232, 2002.

44. Urheim S, Rabben SI, Skulstad H, Lyseggen E, Ihlen H, and Smiseth OA. Regional myocardial work by strain Doppler echocardiography and LV pressure: a new method for quantifying myocardial function. Am J Physiol Heart Circ Physiol 288: H2375-2380, 2005.

45. Wang J, Khoury DS, Thohan V, Torre-Amione G, and Nagueh SF. Global diastolic strain rate for the assessment of left ventricular relaxation and filling pressures. Circulation 115: 1376-1383, 2007. 
In preparation and in part presented at the 2001 Annual Scientific Sessions of the American College of Cardiology.

J Am Coll Cardiol. 2001;37:222A-222A Suppl. A. 
Discussion 


\section{DISCUSSION}

In this chapter, we summarize and discuss the results presented in this thesis, put them into clinical perspective and propose improved algorithms for clinical diagnostics and decision-making.

\section{CARDIAC MECHANOMYOPATHY - EXPERIMENTAL INSIGHTS}

\section{Cardiac mechanomyopathy}

We propose the term "cardiac mechanomyopathy" to indicate the multi-facetted remodeling processes in hemodynamically-overloaded hearts, their underlying mechanical stimuli, and the mutual relations between these stimuli and different functional and structural elements in the myocardium. A schematic illustration of the elements involved in the pathophysiological mechanisms contributing to cardiac mechanomyopathy is depicted in Figure 1.

In the studies for this thesis we investigated the canine heart with chronic complete atrioventricular block (AVB) in order to unravel functional and structural aspects of this model of cardiac mechanomyopathy. The AVB model has a longstanding research history in the field of ventricular hypertrophy and heart failure, which dates back more than 50 years $[13,22,41,110,131,132,150,151,167,197-199]$. Mechanical $[43,44,110,112,198]$, hemodynamic $[37,44,55,101,188]$, structural $[1,42,43,140,141$, $155,178,185,188,197]$ and electrophysiological [140,141,145,146,152,155,175,185,186,188-190] properties have been extensively characterized. The dog with chronic AVB is also an established model of acquired ventricular proarrhythmia: both torsades de pointes (TdP) and delayed afterdepolarization-dependent monomorphic ventricular tachyarrhythmias are readily induced. In the chronic phase, the model exhibits compensated ventricular contractile function, which can be considered a beneficial adaptation to bradycardia-induced overload, whereas the proarrhythmia clearly is a pathological feature.

Apart from the canine model with $A V B$, other canine models of cardiac overload have been described: the aorto-caval fistula model $[8,9,91,94,114,184,192]$, the aortic regurgitation model $[49,51,56,93,170,195]$, the mitral regurgitation model $[27,40,81,119,149,154,169,170,201]$, the aortic stenosis/banding model $[26,27,54,67,71,85,153,158]$, the coronary microembolization/ occlusion model $[87,136-138]$, ventricular pacing models $[1,124,126]$, the left bundle branch block model [182], the experimental hypertension model [17,87], and the rapid ventricular pacing model $[4,11,28,92,105,118,148,192]$. In almost all of these models, cardiac mechanics have been addressed, e.g., for aorto-caval fistula see references [8,9,111], aortic regurgitation $[51,56,170]$, mitral regurgitation $[27,40,76,119,149,170,201)$, aortic stenosis/banding $[27,54,67,71,85,158]$, 


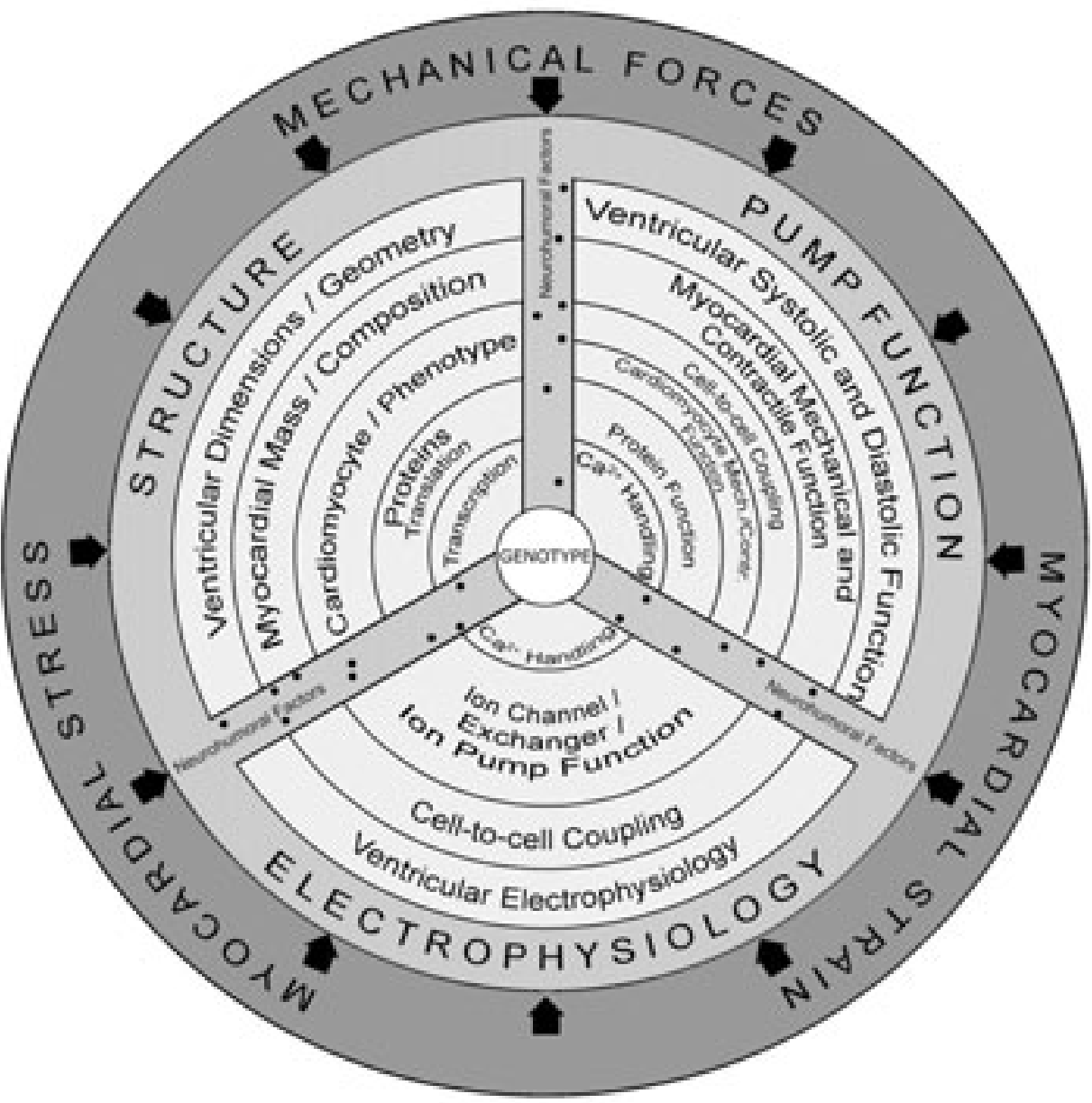

Figure 1 | Cardiac mechanomyopathy. Schematic illustration of cardiac mechanomyopathy composed of interacting structural and functional elements, from the (sub-)cellular to the organ level, all (in-)directly influenced by the primary stimuli, i.e., the mechanical forces imposed on the myocardium. Note that all elements of the scheme may have mutual interrelations. The relative importance of various elements depends on the individual constitution, the genotype, the mechanical stimuli imposed on the system, and their mutual interrelations may change dynamically over time. 
ventricular pacing [124-126,174], and left bundle branch block [182]. Yet, in these publications, mechanical stimuli are mostly related to LV geometry and the degree of hypertrophy. Studies that relate mechanical stimuli to more in-depth analyses of the myocardial structure or function are scarce $[9,27,40,85,114,119,158]$.

This lack of literature regarding the integration of mechanical, structural and functional data also holds for human cardiac disease due to mechanical overload. The first descriptions of mechanical stimuli imposed on the human heart during various pathologies date back many decades $[73,122,123,139]$ and are still pursued $[82,107,125,171]$. Yet, as holds for the experimental models mentioned above, integrative studies focusing on the link between mechanical stimuli and myocardial remodeling are scarce in the literature. However, such studies are necessary to enhance mechanistic understanding of overload-induced cardiac remodeling.

\section{CARDIAC MECHANOMYOPATHY IN THE CANINE MODEL WITH ATRIOVENTRICULAR BLOCK}

\section{Assessment of cardiac mechanical overload}

In the studies described in Chapter $\mathbf{3}$ and Chapter $\mathbf{6}$ we analyzed the mechanical stimuli acting on the myocardium in the canine AVB model by using a validated mathematical model of cardiac mechanics [5]. This partly invasive technique, incorporating LV intracavitary pressure measurements, is further developed in Chapter 7 resulting in a completely non-invasive approach applicable in humans [6].

For the canine AVB model, we found that diastolic myofiber stress and ejection strain, but not systolic stress, are importantly altered early after induction of bradycardia-induced overload and these changes coincide with peak hypertrophic growth. Other studies using MRI, echocardiography and conductance catheters $[52,82,159,178,188]$ confirm these findings in experimental dogs and humans with AVB.

In general terms, stress and strain are the only mechanical factors known to exist in physics. They are known to be important stimuli for hypertrophic remodeling in the heart, as described in other animal models of volume overload $[47,48,72,97,98,113,200]$. In most studies only maximal and/or minimal values of stress and strain are addressed during the cardiac cycle. Yet, many other potentially important parameters can be derived from continuous signals of stress and strain throughout the cardiac cycle $[72,113]$. E.g., it is possible to analyze the peak changes relative to mean changes, the relative changes, the rate of changes, the cumulative changes, i.e., the integrated sum of a parameter in a specific time interval. At this moment we do not know whether and to what extent these derived parameters influence myocardial remodeling. In the canine AVB model, the cardiac 
cycle is characterized by bradycardia and an independent contraction of atria and ventricles. In this situation, continuous assessment of cardiac mechanics could be helpful to explore the potential role of mechanical stimuli during a prolonged diastolic phase and the role of asynchronous atrial contractions resulting in numerous independent 'atrial kicks' during different phases of diastole [108].

The absolute values of myofiber mechanics obtained in the AVB model were comparable with those described in-vitro by others [39]. Contemporary technology enables to quantify mechanics even at the (sub)cellular level using magnetic microbeads or carbon nanofiber arrays $[12,75,90]$. These novel approaches allow to further assess the validity of our data in experiments on (sub-)cellular mechanics in vitro.

Importantly, the computational models of myocardial mechanics used in this thesis assume a homogeneous distribution of mechanical forces within the heart, as described in Chapter 3. For this purpose, we used the 1-fiber model in which the heart consists of a single myocardial band $[2,24,31,45,100,164,165]$. Others reported that ventricular mechanics may vary within the LV wall, e.g., transmurally $[7,9,103,127,171]$, which should be accounted for in future studies, e.g., by using MRI tagging. This technique allows transmural as well as a regional assessments of myocardial strains.

\section{Assessment of myocardial remodeling}

We described a novel approach enabling serial sampling of large LV myocardial specimens (needle biopsies) in a minimally-invasive way, as detailed in Chapter 4. This percutaneous closed-chest approach for intramural LV biopsy sampling shares many advantages with the clinically well-known catheter-based endomyocardial biopsy technique, but results in larger samples of good quality. The specimens extend from the epicardium to deep into the midmyocardium, as illustrated in Figure $3 b$ and $3 c$ in Chapter 4. This novel approach is likely also suitable for other large animal models and potentially in anesthetized humans under very controlled conditions. The relatively large-sized tissue samples can be used for various cell-biological and molecular analyses, in parallel to the assessment of mechanical and other functional parameters.

\section{Electrical remodeling}

Applying the serial biopsy-technique, we described a profound decrease of the $\mathrm{K}^{+}$channel subunit KCNQ1 that occurs within days after AVB induction and is followed by a more gradual decrease of $B_{1} A R$ expression. It could be concluded that the downregulation and blunted $B$-adrenergic activation of the $\mathrm{K}^{+}$channel $\mathrm{I}_{\mathrm{Ks}}$ contribute to the loss of $\beta$-adrenergic-induced shortening of ventricular repolarization, which favors proarrhythmia. Provocation testing with isoproterenol identified the repolarization instability based on the acquired ion-channelopathy, as detailed in Chapter 5. The time course of these processes had not been studied before using individual animals as 
their own controls. Based on these findings, the mutual relation between neurohumoral alterations and electrophysiological changes occurring over time could now be related to other functional and structural changes in this model, e.g., the mechanical assessment and structural myocardial remodeling. Such an integrative analysis likely provides further mechanistic clues that are also relevant for humans with overload-induced proarrhythmia.

\section{Structural remodeling}

In the canine AVB model myocardial remodeling involves mainly the cardiomyocytes, whereas, based on immunohistochemical studies, the non-cardiomyocytes and the interstitium are less affected. Ventricular myocardial hypertrophy is largely determined by cellular lengthening [185], although data on the possible loss of cardiomyocytes (apoptosis, necrosis?) during AVB are lacking. The extracellular matrix volume and the collagen volume fraction of around $4 \%$ were found to be unaltered $[121,174,188]$. In addition, the immunoexpression of vimentin, a fibroblast marker, and the extracellular matrix protein laminin were not found to be altered during AVB compared to control dogs with sinus rhythm, as shown in Chapter $\mathbf{4}$ and Chapter $\mathbf{6}$. It should be noted that only one single study has reported that the volume of collagen fibers and extracellular space can be increased during chronic AVB [155]. Only semi-quantitative data were given based on a limited group comparison in beagle dogs [155].

In Chapter 6, we also studied cardiomyocyte cytoskeletal remodeling, including key proteins involved in mechanosensing and -transduction, in relation to increased mechanical stimuli. We found that acute volume overload after AVB causes a transient compromise of cytoskeletal integrity, which is based, at least partly, on transcriptional downregulation. Structural reorganization and a strong drive to compensated hypertrophy are attended by the upregulation of melusin and MLP, known as procompensatory proteins in other models of hypertrophy $[3,19,36,68,69,84]$.

In order to gain more insights into how the mechanical changes in this model are converted to altered gene expression, we performed experiments analyzing key regulatory proteins downstream of $\beta_{10}$-integrin and melusin, i.e., Akt (protein kinase $B$ ) and glycogen-synthase-kinase-3 $\beta$ (GSK3 $\beta$ ), and their phosphorylated (P) forms. From the literature it is known that an increased ratio of P-Akt/ Akt stimulates the phosphorylation of GSK3 $\beta$. An increased ratio of P-GSK3 $\beta / G S K 3 \beta$ stimulates hypertrophy [66]. We studied the ratiometric expression of P-Akt/Akt and P-GSK3 $\beta / G S K 3 \beta$ in serial biopsies from dogs at sinus rhythm and over time from acute to chronic AVB. Interestingly, the significant increase of both ratios in the second week corresponds exactly with the moment of peak hypertrophic growth, which suggests that the sequential interactions of $\beta_{1 D}$-integrin, melusin, Akt/P-Akt and GSK3 $\beta / P-G S K 3 \beta$ could form a central axis of hypertrophic signaling in this model. GSK3 $\beta$ also stimulates the phosphorylation of glycogen synthase [32]. This enzyme promotes glycogen synthesis whereas the phosphorylated form inhibits it. A relative decrease of GSK3 $\beta$, as during mechanical stimulation when the balance of GSK3 $\beta$ and P-GSK3 $\beta$ tips towards P-GSK3 $\beta$, 
may thus lead to increased glycogen synthesis and could form the basis of the glycogen data shown in Chapter 6.

The biological impact of the increased melusin levels is further supported by analyzing melusininteracting $\beta_{1 D}$-integrin in conjunction with its directly interacting extracellular matrix protein laminin [135]. Whereas $\beta_{1 D}$-integrin was significantly and persistently upregulated from 3 days AVB onward, the immunoexpression of laminin was found to be unaltered. These findings suggest that the cardiomyocyte protein $\beta_{10}$-integrin, and not the extracellular matrix protein laminin, is among the first upstream elements activated by mechanical stimuli.

Moreover, we studied the LIM proteins MLP and fhl2, both involved in mechanosensing and -transduction in cardiac hypertrophy and failure $[79,84,96]$. In our study, we found a different temporal expression pattern for MLP and fhl2. Based on the data available in the literature we consider the following: both MLP and fhl 2 contain a LIM-domain motif, which allows multiple proteinprotein interactions and constitutes the structural basis for functional diversity [80]. Importantly, the protein structures of MLP and fhl2 differ: MLP is a cysteine-rich LIM protein (a subclass of the LIM-only proteins), whereas fhl2 is part of the four-and-a-half LIM protein family [80]. The partly divergent protein structures of MLP and fhl 2 are compatible with a different functional role. For example, in overload-induced cardiac hypertrophy MLP is considered to be prohypertrophic $[68,69]$ whereas fhl2 is regarded as a repressor of hypertrophy [129]. MLP mediates anchorage of calcineurin to the Z-disk, thus playing an important role in the activation of the calcineurinNFAT pathway [69]. In contrast, fhl2 interacts with ERK2, an element of the MAP-kinase signaling pathway. ERK2 interacts with fhl2, but it does not interact with MLP, which underscores the protein-specific action of LIM proteins [129]. Hypertrophic responses induced by $\alpha$-adrenergic stimulation with phenylephrine, and mediated via ERK2, are partially antagonized by fhl2, which attenuates transcription [129]. Moreover, fhl2-null mice, which exhibit a normal cardiac phenotype, also show increased hypertrophic growth after $\beta$-adrenergic stimulation with isoproterenol, supporting repressor-like activity of fhl2 in the overloaded myocardium [86].

The early transient downregulation of fhl 2 coincides with enhanced adrenergic tone as evident from Chapter 5, and maximum hypertrophic growth in the first 10 days after AVB [44]. It could well be that hypertrophy is partly caused by an enhanced $\alpha$ - and $\beta$-adrenergic stimulation early after induction of AVB and mediated via MAP kinase/ERK2. The latter pathway could be incompletely repressed by the fhl2 reduction at this stage, promoting hypertrophy. Whether these mechanisms apply indeed in the canine model of AVB remains to be established.

In dogs with $A V B$, the mechanical stress-sensor function of MLP appears to be intact given the observed BNP release [84], which is known to be regulated via the calcineurin-NFAT pathway [106]. The steady increase of MLP protein levels parallels the increase of ventricular mass, suggesting a prohypertrophic action of MLP. In order to explore the mechanism underlying the actions of MLP, we performed experiments using immunohistochemistry to study the localization of MLP in the myocyte at control and in chronic AVB. At control we found a faint cytoplasmic staining pattern, which partly exhibited cross-striations, as described by Ecarnot-Laubriet et al. [3,46]. Immunola- 
beling of MLP in the nucleus was also faint. During chronic AVB, increased cytoplasmic labeling was observed (compared to control) and the nuclei exhibited strong MLP-positive labeling. These data showing a cytoplasmic upregulation along with a nuclear relocation of MLP during hypertrophy in this model suggest a dual, i.e., cytoplasmic and nuclear, functional role for MLP during mechanical overload in this model.

\section{Integrating the picture - potential links between mechanical stimuli \& structural, electrical, and contractile remodeling}

In the AVB model, changes of systolic and diastolic mechanics are most pronounced in the first weeks of AVB. These increased mechanics likely form the basic stimuli for the coincident structural and functional, i.e., electrical and contractile cardiomyocyte remodeling, as illustrated in Figure 2. In this early period, the cytoskeletal integrity is temporarily distorted. Ultimately, pro-compensatory mechanisms prevail and compensated hypertrophy ensues. In this context, transcriptional downregulation seems to be more important than posttranslational modification (at least for the specific processes investigated), but this issue deserves further investigation.

Based on the results from Chapter 5, cross-talk between the cytoskeleton and certain ion channels could be well possible in the AVB model. KCNE1, the regulatory $\beta$-subunit of the $I_{\mathrm{Ks}}$ channel, which co-assembles with the $\alpha$-subunit KCNQ1, specifically interacts with the sarcomeric Z-disk protein T-cap (telethonin) in the Z-line region [53]. T-cap is well known to interact with the N-terminus of the giant protein titin [57-64,95]. This interaction might well contribute to mechanically-induced electrical alterations in the sense of mechano-electrical feedback [53]. Furthermore, together with MLP T-cap and titin form a 'mechanosensing machinery' [84], which appears to be intact in the canine AVB model as pointed out in Chapter 6. It remains to be established whether the KCNE1-T-cap link has any mechano-electrical function in the AVB dog to affect $I_{\text {Ks }}$ function. In addition, the cardiac-enriched four-and-a-half LIM domain-containing protein (fhl2) was identified as potential KCNE1-interacting partner $[88,89]$. The transient decrease of fhl2 during AVB also parallels the electrical remodeling process, which makes fhl 2 a potential mediator protein in terms of mechanical overload-induced electrical remodeling.

Besides potential physical links between cardiomyocyte electrical elements and the cytoskeleton, altered cellular $\mathrm{Ca}^{2+}$ handling is well known to play an important pathophysiological role in overload-induced cardiac hypertrophy and failure. In the canine AVB model, it is characterized by enhanced $\mathrm{Ca}^{2+}$ release from the sarcoplasmic reticulum and enhanced $\mathrm{Na}^{+}-\mathrm{Ca}^{2+}$ exchange [145]. Increased subsarcolemmal $\mathrm{Na}^{+}$concentration may cause the $\mathrm{Ca}^{2+}$ changes [177]. These observations related to altered $\mathrm{Ca}^{2+}$ handling in this model all contribute to (over-) compensated contractility and mechanical overload, i.e., systolic strain, but also to proarrhythmia $[144,146,147]$. In addition, increased diastolic $\mathrm{Ca}^{2+}$ load may contribute to $\mathrm{Ca}^{2+}$-dependent diastolic dysfunction and might thereby be related to altered diastolic mechanics [117]. 
CHAPTER 8 | DISCUSSION

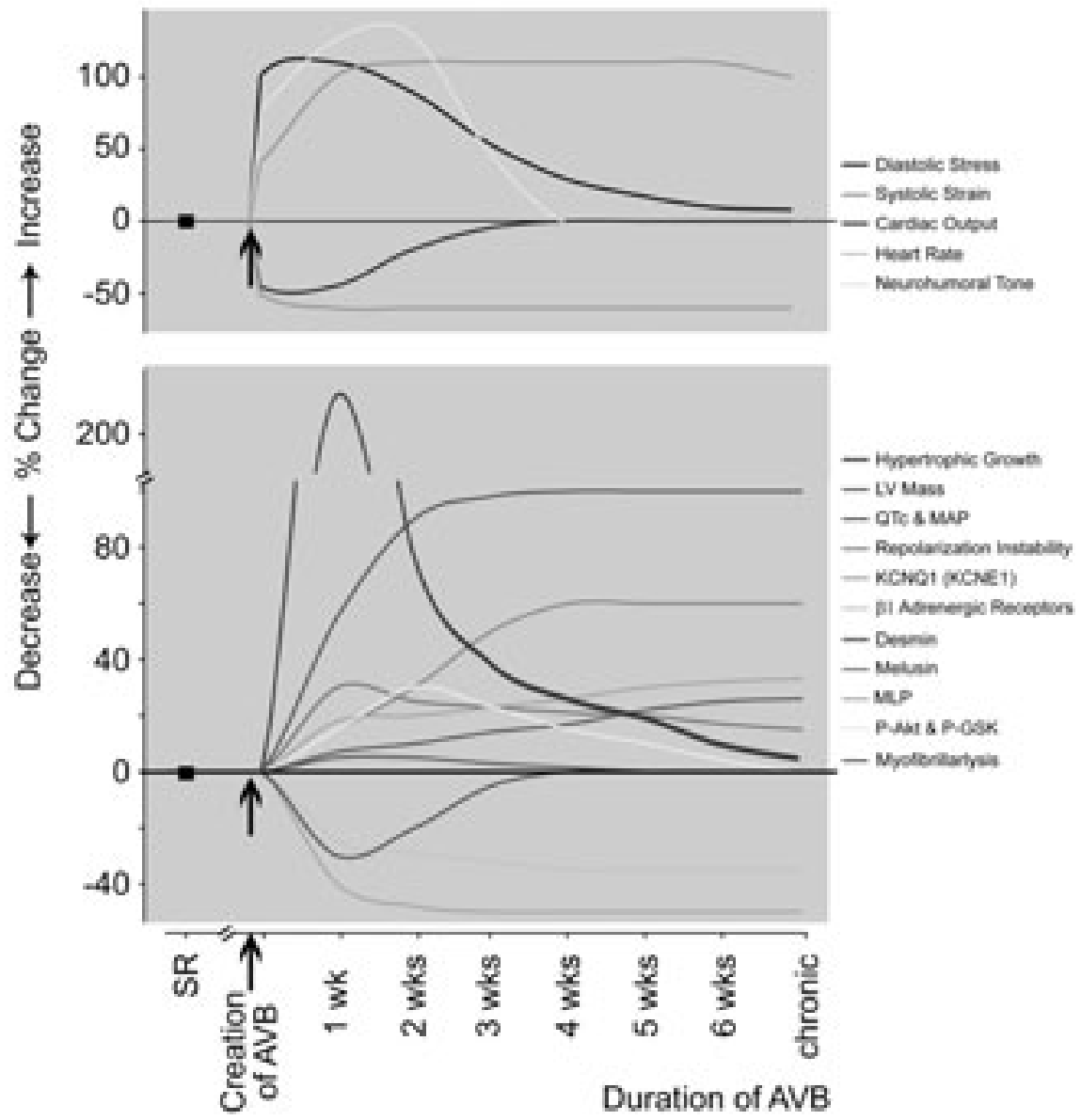

Figure 2 Time course of cardiac mechanics and related remodeling in the AVB model. Schematic illustration of cardiac mechanics, hemodynamics and neurohumoral tone upon creation of AVB in temporal relation to different functional and structural myocardial remodeling processes. For full colour illustration, see page 229. 
Moreover, $\mathrm{Ca}^{2+}$ handling is important for the function of many proteins involved in hypertrophymediating pathways.

Myocardial remodeling in the AVB model may also be importantly mediated by adrenergic mechanisms. We and others have found, as reported in Chapter 5, that norepinephrine plasma levels are clearly elevated during AVB, i.e., specifically in the first 2-4 weeks $[159,188]$. Increased catecholamine levels likely enhance vascular resistance and contractility, and thereby contribute to mechanical overload. Besides, increased adrenergic tone alters $\mathrm{Ca}^{2+}$ homeostasis in cardiomyocytes, e.g., $\beta$-adrenergic stimulation can dynamically enhance cellular $\mathrm{Ca}^{2+}$ entry and loading [187].

In order to identify and characterize the assumed primary stimuli for functional and structural myocardial remodeling in the canine AVB model in more detail a number of interventions should be investigated in future experiments. Restoration of the low ventricular rate by pacing is an obvious intervention, since the bradycardia has been shown to be an important stimulus for remodeling processes after AVB $[121,156]$. In general, cardiac pacing allows to restore a physiological heart rate as well as the $A V$-synchrony. It has been shown, that single site pacing from the $R V$ is at least able to partially reverse the LV hypertrophy, whereas electrical remodeling could not be reversed [121]. Others have demonstrated that biventricular pacing is superior to RV pacing in the canine AVB model, at least in acute conditions [52]. This might also hold for the chronic setting but deserves further analysis. Yet, in one study 8 weeks of biventricular pacing did not reverse electrical remodeling after chronic AVB [120].

Recently, a more physiological restoration of the altered ventricular activation pattern was achieved by high-septal pacing. In this study, the paced ventricular activation is virtually physiological whereas the bradycardia is similar to AVB. This mode of pacing led to a reduction of ventricular electrical remodeling and proarrhythmia in dogs after 4 weeks of $A V B$, whereas the hypertrophy as well as the compensated contractility were comparable to non-paced AVB dogs with idioventricular rhythm [196]. These findings suggest that the altered ventricular activation during AVB contributes to electrical remodeling and proarrhythmia. In future studies, the contribution of AV dyssynchrony should be addressed, since it is well known to potentially alter ventricular geometry and hemodynamics [108]. AV dyssynchrony could induce chronic mechano-electric alterations. Spatial heterogeneity of the mechanical stimuli may be of crucial importance for myocardial remodeling, but it remains to be determined whether an exaggerated spatial heterogeneity of ion-channel expression during cardiac overload is also the result of this. The issue of spatial heterogeneity of mechanical stimuli has not been addressed in this thesis due to the fact that we applied methods to assess global, not regionally different, LV mechanics. In order to address this aspect, techniques capable of assessing spatial distributions of mechanical stimuli and concomitant myocardial remodeling are necessary, e.g., as has been applied by Omens et al. [7]. These investigators used invasive techniques in normal dogs to analyze LV myocardial strain and repolarization transmurally by inserting bead markers in conjunction with plunge electrodes. They found that the onset of myofiber shortening (earliest at the endocardium) and relaxation (earliest at the epicardium) are characterized by a transmural dispersion of mechanics, whereas no dispersion of repolarization could be found. It 
remains to be determined if these findings also hold for conditions of mechanical overload, as in the AVB model [7].

An enhanced mechanistic understanding and the identification of primary stimuli responsible for myocardial remodeling in the AVB model is closely related to clinical problems in cardiac pacing and resynchronization therapy. In this evolving field of heart-failure therapy current experimental and clinical studies address issues as the optimal patient selection, choice of pacing site and timing of pacing using myocardial deformation analysis, hemodynamic and electrocardiographic methods with respect to favorable structural, contractile and electrical reverse remodeling [14-16,18,157,168,176,179-183].

Besides ventricular pacing, as mentioned above, other interventions could be helpful to beneficially alter ventricular mechanics and to further unravel the underlying pathophysiology of the cardiac mechanomyopathy during AVB. For example, surgical techniques to obtain passive containment of the heart or cardiac assist devices could be applied to mechanically unload the ventricles. This has been shown to influence different elements of remodeling in overloaded ventricles in various pathologies $[10,29,30,50,65,83,115,130,160]$. Pharmacological interventions [140], which have also been shown to interfere with remodeling, could also be extended and (re-)evaluated in terms of mechano-structural-functional phenotyping.

Yet another method to gain more mechanistic insights in overload-induced remodeling is the use of computational modeling. In recent years various models have been found suitable to simulate complex phenomena related to ventricular electrophysiology and mechanics $[6,33,34,99,162,166]$. Yet, integrated three-dimensional models of the human heart incorporating 1) depolarizing and repolarizing electrophysiological properties from the level of the ion channels to electrocardiographic representation on the body surface, 2) regional and transmural mechanics acting throughout the cardiac cycle, and 3) structural and architectural elements ranging from the cellular to the organ level, are still under development [74].

\section{CARDIAC MECHANOMYOPATHY - CLINICAL IMPLICATIONS}

\section{Patients with cardiac mechanomyopathy}

In this section, we illustrate 2 clinical cases with cardiac overload and multifaced phenotypic aspects, which potentially share common mechanisms. The clinical course of both patients illustrates that many aspects of cardiac mechanomyopathy are currently unresolved. This is mainly due to an incomplete mechanistic characterization of the underlying pathophysiology, which contributes to the shortcomings of contemporary diagnostics in clinical cardiology. Basically, the myocardium cannot be comprehensively characterized with regard to molecular composition (cytoskeleton, 
myofilaments, ion-channel expression) in relation to contractile and electrical functions. Moreover, the roles of mechanical and neurohumoral factors as stimuli for remodeling remain largely elusive. Yet, improved mechanistic understanding in individual patients remains a prerequisite for the development of etiology-based therapy.

Case 1: In February 2007, a 46-year old male was transferred to the Department of Cardiology of the Academic Hospital Maastricht. He had been healthy before, but complained about progressively decreasing exercise tolerance, palpitations and (near-)collapses in the months before presentation. Clinical assessment revealed symptomatic ventricular extrasystoles, an episode of sustained atrial fibrillation, and most importantly a type-Il atrial septal defect (ASD) with hemodynamically relevant left-to-right shunting requiring surgical closure. Based on the literature, this case is not exceptional at first glance, as an atrial septal defect can become symptomatic at late age [191].

However, as a rather exceptional additional clinical problem the patient developed monomorphic nonsustained ventricular tachycardia (VT) originating from the right ventricular (RV) outflow tract. This was first documented upon introduction of anesthesia while in the operating room awaiting thoracotomy and surgical closure of the ASD. The procedure and the postoperative course were unremarkable. Postoperatively the patient felt better and had improved exercise capacity right away. Yet, frequent symptomatic episodes of the same monomorphic VT persisted. Oral administration of metoprolol reduced the occurrence of VT at rest, but did not prevent recurrences during exercise testing. Clinical work-up showed no significant coronary artery disease and echocardiography demonstrated a preserved LV function and a slightly diminished RV function (tricuspid annular plane systolic excursion was $11 \mathrm{~mm}$ ) in the setting of enlarged RV dimensions [43 $\mathrm{mm}$ ). An electrophysiological study was performed using multi-site mapping, which revealed a rather large arrhythmogenic area. Radiofrequency catheter ablation was applied, yet in the days after the procedure the $V T$ persisted under $\beta$-blockade, especially during exercise testing. The family history did not reveal sudden death, or cardiomyopathy and cardiac MRI did not indicate arrhythmogenic RV cardiomyopathy. Given the persistence of symptomatic, fast VT (intermediate axis, left-bundle-branch-block configuration, cycle length $300 \mathrm{~ms}$ ) under $\beta$-blockade and after radiofrequency ablation, at 4 weeks after surgical closure of the ASD type II, it was decided to implant an internal cardioverter defibrillator (ICD). See Figure 3 for the illustration of echo- and electrocardiographic findings.

This case illustrates the complexity of mechanical-overload-induced cardiac disease. Questions arise as to whether mechanical factors and related structural and functional myocardial remodeling could form the basis for the VT; how potential interrelations between these different aspects of the disease are established; and how they should be treated, preferentially based on the underlying mechanisms. In this sense, the clinical case underscores the need for an integrative diagnostic approach, which is not yet sufficiently applicable in current clinical practice.

In this case, monomorphic VT (cycle length (CL) 300ms) arises from the RV, initiating with a long coupling interval (CL 520ms). The VT is probably triggered by sympathetic stimulation (exercise, emotional stress). 
Diastole
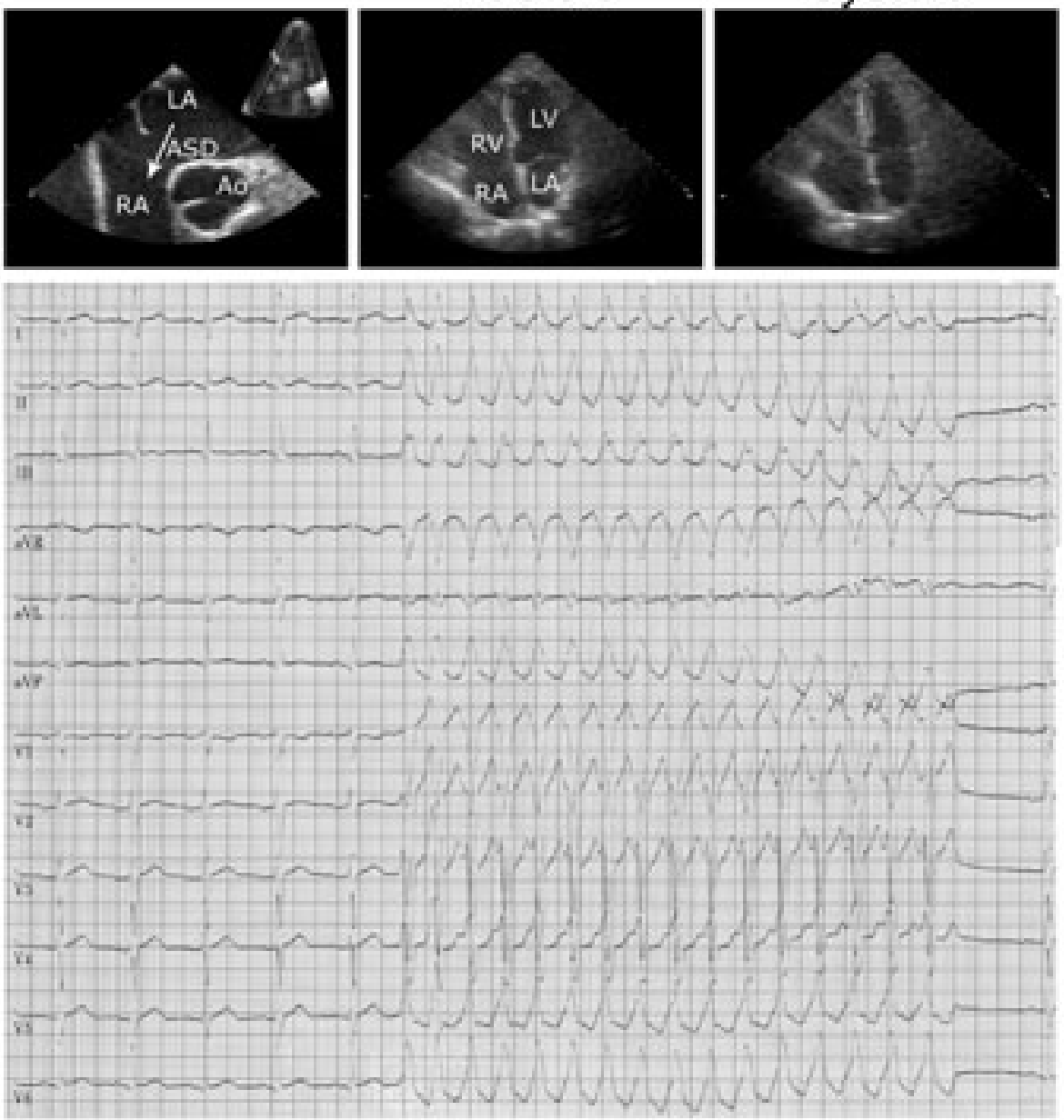

Figure $\mathbf{3}$ | Cardiac mechanomyopathy - Case 1. Transesophageal echocardiography (top, left panel) demonstrating ASD type II (arrow) with important left-to-right shunting (insert, color Doppler flow). Ao indicates aortic valve, LA and RA indicate the left and right atrium, respectively. Transthoracic echocardiographic 4-chamber views after surgical correction of the ASD (top, mid and right panel) showing a somewhat dilated RV and a normal LV during diastole (top, mid) and systole (top, right). 12-Leads electrocardiogram (bottom) 10 days after surgical correction of the ASD showing SR (left) and VT (right) originating in the RV outflow tract. See text for details. For full colour illustration, see page 230. 
Priori et al. were among the first to provide experimental evidence for a role of triggered activity, i.e., delayed afterdepolarizations (DADs) occurring in vivo during a condition of cardiac overload [128]. In cats with aortic constriction, DADs could be induced by $\mathrm{Ca}^{2+}$ loading, digitalis, and sympathetic stimulation [128]. The long-coupled initiation of the VT in Case 1 is indeed compatible with DADs as initiating mechanism. This is also supported by successful initiation of the clinical VT by programmed electrical stimulation using pacing at increasingly fast rates. Yet, the results of electrophysiological testing should always be interpreted with caution when searching for the initiating and perpetuating mechanisms of a VT [134]. Other arrhythmogenic mechanisms cannot be ruled out completely.

Based on the findings in this thesis, it could be that the RV myocardium of the patient in Case 1 has undergone electrical remodeling as a consequence of the mechanical overload imposed on the RV due to the longstanding ASD. This could have manifested as enhanced cellular $\mathrm{Ca}^{2+}$ release and/or enhanced $\mathrm{Na}^{+}-\mathrm{Ca}^{2+}$ exchange in the setting of hypertrophy, promoting VT under adrenergic stimulation. Our group has previously reported that ventricular ectopy and VT are DAD-mediated during fast ventricular pacing and $\mathrm{Ca}^{2+}$ overload induced by digitalis [38].

In Case 1, electrical remodeling may also encompass spatial heterogeneity of electrical activation and repolarization between the ventricles (LV versus RV), because the mechanical overload and subsequent remodeling processes apply to the RV and not to the LV.

Clinical work-up in Case 1 includes the quantification of the atrial left-to-right shunt, the ventricular hemodynamics, the contractile function and the cardiac dimensions. The decision whether or not to close the shunt is based mainly on empirical data $[35,191]$. A causal analysis of the relation between cardiac overload and ventricular proarrhythmia is hard to establish based on the limitations of currently available diagnostic tests. The risks of sticking to these tests is nicely phrased in the following analogy:

'If the only tool you have is a hammer, you tend to see every problem as a nail.'

Abraham Harold Maslow, clinical psychologist (* 1-4-1908 - +8-6-1970)

Efforts should therefore be directed towards developing improved diagnostic modalities in individual patients.

The above-mentioned case illustrates the clinical relevance of mechanical overload in the RV. In practice, acquired disease of the LV occurs much more frequently. Therefore, the next case illustrates LV mechanomyopathy:

Case 2: In May 2007, a 67-year old male was transferred to the Department of Cardiology of the Academic Hospital Maastricht after he had suffered an unwitnessed in-hospital cardiac arrest due to ventricular fibrillation. He was successfully resuscitated. Five days before the resuscitation he had been admitted because of 
dyspnea based on a combined problem of exaggerated chronic obstructive pulmonary disease (COPD) due to pneumonia, congestive heart failure and atrial fibrillation with a high ventricular rate. He was appropriately treated with antibiotics, i.e., amoxicilline/clavulanic acid, diuretics and digoxin. Adequate rate control had been reached before he suffered the cardiac arrest. Kidney function and electrolytes were within normal limits. His past medical history revealed COPD, diabetes mellitus type II, a limited old inferior myocardial infarction and successfully revascularized 2-vessel coronary artery disease (right and circumflex coronary artery) as confirmed by coronary angiography. There were no any signs of a new ischemic event. Systolic LV function was slightly diminished (EF 45\%). After resuscitation he was intubated, mechanically ventilated and treated with piritramide, midazolam and amiodarone intravenously to control the recurrent high ventricular rate during atrial fibrillation. Despite this treatment he suffered relapsing polymorphic VT (Figure 4). When mechanical ventilation and anti-arrhythmic therapy were discontinued the polymorphic ventricular arrhythmias finally stopped and no recurrences were observed during the subsequent clinical observation period.

In this case, polymorphic VT, i.e., acquired torsades-de-pointes (TdP) arrhythmias, occurred in a setting of LV disease with overload, i.e., slightly diminished systolic LV function in a patient with a limited old myocardial infarction and atrial fibrillation with a high ventricular response.

It remains unclear, which are the mechanical triggers in this case and to what extent overloadinduced electrical remodeling plays a role. From the ECG and the echocardiogram the old inferior wall myocardial infarction seems too small to explain major mechanical overload leading to significant electrical remodeling. It is of course possible that longstanding atrial fibrillation caused a tachycardiomyopathic component contributing to impaired LV contractile function.

As in Case 1, many aspects of a cardiac mechanomyopathy apply to this case. Yet, the underlying mechanisms responsible for the arrhythmogenesis remained elusive, which again precluded mechanism-based individualized treatment. Although the phenomenon of acquired TdP is well-described $[25,102,109,133,163,202]$ and many conditions (e.g., electrolyte disturbances, heart failure, drug effects, bradycardia, sudden changes of heart rate, etc.) are known to promote their occurrence, the contributing role of short- and long-term mechanical overload and structural and functional myocardial remodeling could not be elucidated. This lack of mechanistic insights, particularly the patient-specific characterization of the underlying pathophysiology, limits appropriate clinical decision-making.

\section{Novel approach to the patient with cardiac mechanomyopathy}

In this section, we translate the results of the thesis into an integrative diagnostic approach towards patients with a cardiac mechanomyopathy. Central to this approach is that myocardial mechanical load is characterized and mechanistically linked to remodeling.

As a first diagnostic step, cardiac mechanical load should be quantified in the individual patient. Ideally, the applied methodology is non-invasive, comprehensive, quick and easy-to-use, widely available, and inexpensive. As outlined in Chapter 7, CircAdapt computational modeling has the potential 

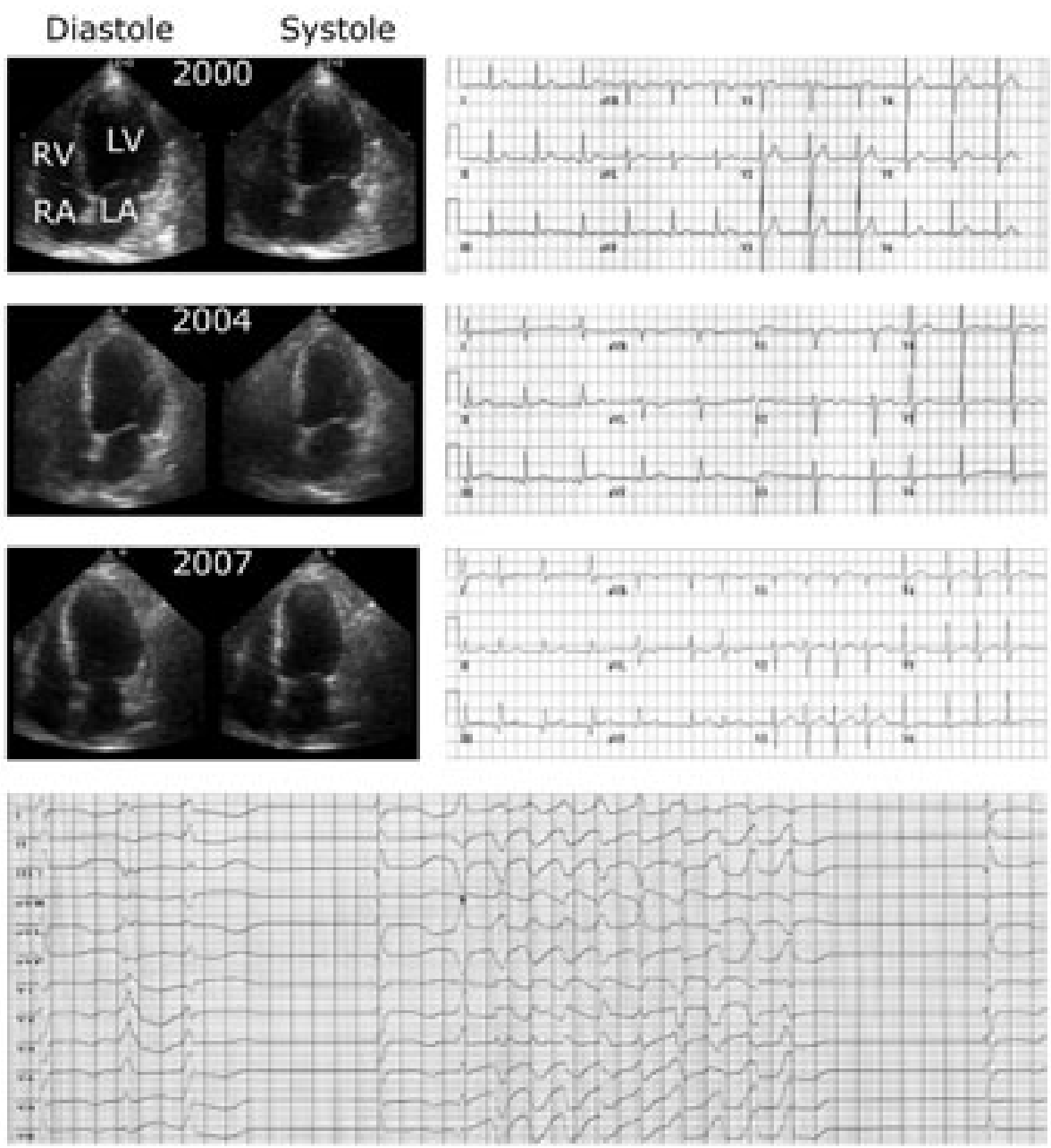

Figure $4 \mid$ Cardiac mechanomyopathy - Case 2. Serial transthoracic echocardiographic 4-chamber views during diastole and systole (left panels) illustrate a somewhat dilated LV (LV end-diastolic diameter $60 \mathrm{~mm}$ ) with a slightly diminished systolic function (EF 45\%), which had been stable through the years. Corresponding 12-lead electrocardiograms (right panels) show signs of an old inferior myocardial infarction. Note the occurrence of a non-sustained polymorphic VT after a short-long-short cycle (bottom panel). See text for details. 
to meet all these criteria. In addition, the use of serum biomarkers of cardiac overload is attractive, as reflected by their widespread clinical use and implications $[21,77,78,116,172,173,193,194]$. Yet, currently available biomarkers are indirect markers of load and less specific than a comprehensive analysis of mechanical load itself, which is assumed to be a primary stimulus.

In individual patients mechanical forces acting on the myocardium vary dynamically over time, as shown experimentally and clinically in Chapter 3, Chapter 6 and Chapter 7. In addition, the individual cardiac phenotype evolves gradually as the result of mutual interactions between mechanical stimuli, responsive remodeling and the genetic make-up. Serial analysis of cardiac mechanics is preferable over a single measurement. Moreover, serial mechanical phenotyping allows an assessment of individual load history. The term load history suggests that the myocardium has a memory to store the maximum previous load in line with the strain softening property of rubber elastomeres [48]. The structural basis of myocardial strain softening has been demonstrated to be based on damaged collagen ties between interlaminar myofiber sheets caused by increased filling pressures, i.e., myocardial overstretch [48]. In this sense, strain softening and load history have been proposed as important triggers for myocardial remodeling [48].

In Chapter 3, Chapter 5 and Chapter 6 we have shown that (mal)adaptive structural and functional alterations of the myocardium can be transient. Moreover, it has long been known that mechanical overload has time-dependent effects on the myocardium, e.g., transient overload may lead to beneficial alterations, whereas permanent overload is usually detrimental [104]. Thus, besides serial assessment of the load history, serial phenotyping of the myocardium could also be helpful to characterize individual patients. It is obvious that serial myocardial biopsies are not attractive as a general diagnostic technique for large numbers of patients, yet, non-invasive techniques to characterize functional and structural aspects of the myocardium are well-suited for serial analyses. These non-invasive approaches would be particularly useful if clear links between the myocardial phenotype and the non-invasive marker could be established. In this context, the CircAdapt approach presented in Chapter 7 offers promising perspectives. For instance, if specific quantitative mechanical stimuli are closely related to well-defined aspects of adverse myocardial remodeling, mechanical phenotyping alone could be sufficient to monitor patients with mechanical overload.

To better determine the impact of mechanical load on myocardial remodeling it is unavoidable to analyze myocardial biopsies from individual patients. As an initial step, we investigated this in patients with ventricular overload due to valvular disease applying techniques and molecular markers that were successfully studied in experimental animals, as described in Chapter $\mathbf{4}$ and Chapter 6. We used LV intramural biopsies from patients undergoing valvular surgery. All patients had clinically compensated or slightly decompensated ventricular hypertrophy and underwent a routine pre-operative clinical assessment including echocardiography and coronary angiography to exclude significant coronary artery disease. During open-heart surgery intramural needle biopsies were taken from the LV free wall. All patients were electively scheduled for valvular surgery according to current guidelines and gave informed consent to the study. The Medical Ethics Committee of the 
Table 1 | Patients characteristics. AS indicates aortic stenosis, AR, aortic regurgitation, MR, mitral regurgitation, LVEDD, LV end-diastolic diameter, and LVWT, LV wall thickness

\begin{tabular}{|c|c|c|c|c|c|c|c|c|}
\hline \multirow[b]{2}{*}{ Patient } & \multicolumn{5}{|c|}{ Clinical Characteristics } & \multicolumn{3}{|c|}{ Echocardiography } \\
\hline & Age & Gender & NYHA class & Valvular disease & Hypertension & LVEF (\%) & LVED (mm) & LVWT (mm) \\
\hline 2693 & 56 & $M$ & 1 & AS/AR & & 48 & 64 & 13 \\
\hline 8914 & 71 & $\mathrm{~F}$ & $\|-I\|$ & MR & + & 66 & 52 & 9 \\
\hline 1597 & 68 & $\mathrm{~F}$ & $|-| \mid$ & AS & + & 70 & 50 & 12 \\
\hline 8305 & 62 & $M$ & $\|$ & MR & & 49 & 60 & 12 \\
\hline 7338 & 70 & $\mathrm{~F}$ & $\|$ & AS & & 70 & 48 & 11 \\
\hline 3247 & 59 & $M$ & $\|-1\|$ & $\mathrm{AR} / \mathrm{MR}$ & & 70 & 56 & 10 \\
\hline 3169 & 52 & $F$ & $\|-1\|$ & $\mathrm{AR}$ & + & 63 & 58 & 11 \\
\hline 1932 & 56 & $M$ & $\|-111$ & MR & & 72 & 62 & 10 \\
\hline 3878 & 73 & $\mathrm{~F}$ & $\|-1\|$ & MR & & 67 & 56 & 10 \\
\hline 8488 & 54 & $\mathrm{~F}$ & 1 & & & 76 & 43 & 8 \\
\hline 8898 & 83 & $\mathrm{~F}$ & $\|-1\|$ & AS & & 59 & 42 & 15 \\
\hline 5392 & 39 & $M$ & 1 & & & 63 & 44 & 8 \\
\hline 1393 & 76 & $\mathrm{~F}$ & $\|$ & AS/AR & & 62 & 51 & 10 \\
\hline 8066 & 53 & $M$ & III & AS & & 61 & 49 & 12 \\
\hline 4108 & 67 & $M$ & $\|-I I I$ & MR & + & 54 & 56 & 8 \\
\hline 9906 & 52 & $M$ & $\|-1\|$ & AS & + & 64 & 51 & 12 \\
\hline 7379 & 36 & $M$ & 1 & $\mathrm{AR}$ & + & 50 & 66 & 10 \\
\hline 1771 & 39 & $M$ & $|-| \mid$ & AS/AR & & 67 & 68 & 12 \\
\hline 8549 & 69 & $F$ & III & AS & & 64 & 46 & 12 \\
\hline 3471 & 64 & $M$ & $\|$ & AS/AR & + & 58 & 46 & 11 \\
\hline 6982 & 66 & $F$ & II-III & MR & + & 60 & 53 & 8 \\
\hline 6565 & 69 & $M$ & $\|$ & AS & + & 34 & 60 & 12 \\
\hline 6537 & 62 & $M$ & III & AS/MR & & & & \\
\hline
\end{tabular}

Academic Hospital Maastricht approved the experiments. The clinical characteristics of all patients are depicted in Table 1.

The clinical decision to perform valvular surgery is generally based on a large body of evidence. Yet, it remains a matter of debate whether the timing of the surgical intervention is always appropriate. It might well be that some patients are operated upon too late or even too early. Currently, there are no diagnostic tests to determine with certainty the optimal timing of surgery in individual patients. Also, it remains undetermined if the myocardial phenotype is characterized predominantly by beneficial or non-beneficial aspects of remodeling and whether and how the patient benefits from the intervention. On theoretical grounds, assessment of pro- and contra-compensatory myocardial 
Table 2 | Myocardial morphometry. Morphometric analysis of LV myocardial biopsy specimens

\begin{tabular}{|c|c|c|c|}
\hline \multirow[b]{2}{*}{ Patient } & \multicolumn{3}{|l|}{ Cellular Characteristics } \\
\hline & Cardiomyocyte diameter $(\mu \mathrm{m})$ & Myofibrillarlysis (\%) & Extracellular matrix volume (\%) \\
\hline 2693 & $31 \pm 6$ & $53 \pm 0$ & $37 \pm 16$ \\
\hline 8914 & $28 \pm 5$ & $44 \pm 0$ & $36 \pm 12$ \\
\hline 1597 & $22 \pm 2$ & $53 \pm 16$ & $29 \pm 10$ \\
\hline 8305 & $41 \pm 7$ & $63 \pm 10$ & $18 \pm 10$ \\
\hline 7338 & $31 \pm 3$ & $43 \pm 10$ & $15 \pm 5$ \\
\hline 3247 & $31 \pm 5$ & $42 \pm 9$ & $25 \pm 11$ \\
\hline 3169 & $28 \pm 4$ & $59 \pm 24$ & $32 \pm 12$ \\
\hline 1932 & $23 \pm 4$ & $37 \pm 12$ & $25 \pm 12$ \\
\hline 3878 & $31 \pm 6$ & $38 \pm 0$ & $18 \pm 8$ \\
\hline 8488 & $20 \pm 4$ & $8 \pm 4$ & $25 \pm 10$ \\
\hline 8898 & $31 \pm 9$ & $14 \pm 9$ & $19 \pm 11$ \\
\hline 5392 & $23 \pm 5$ & $8 \pm 3$ & $13 \pm 6$ \\
\hline 1393 & $33 \pm 7$ & $27 \pm 6$ & $18 \pm 5$ \\
\hline 8066 & $35 \pm 2$ & $60 \pm 20$ & $18 \pm 8$ \\
\hline 4108 & $28 \pm 2$ & $39 \pm 30$ & $22 \pm 10$ \\
\hline 9906 & $30 \pm 1$ & $56 \pm 28$ & $22 \pm 4$ \\
\hline 7379 & $35 \pm 2$ & $75 \pm 19$ & $32 \pm 10$ \\
\hline 1771 & $33 \pm 1$ & $58 \pm 37$ & $18 \pm 0$ \\
\hline 8549 & $28 \pm 1$ & $23 \pm 2$ & $17 \pm 0$ \\
\hline 3471 & $24 \pm 1$ & $80 \pm 0$ & $22 \pm 0$ \\
\hline 6982 & $28 \pm 2$ & $36 \pm 16$ & $14 \pm 2$ \\
\hline 6565 & $27 \pm 1$ & $49 \pm 22$ & $17 \pm 0$ \\
\hline 6537 & $29 \pm 2$ & $60 \pm 6$ & $22 \pm 6$ \\
\hline
\end{tabular}

markers, as applied in Chapter 4 and Chapter 6, may aid in the clinical decision making and could be complimentary to currently used clinical parameters.

We characterized the LV intramural biopsy samples using light and electron microscopy to assess the myocardial (ultra)structure, the degree of cellular hypertrophy and the extent of extracellular matrix alteration (Table 2). In addition, we studied a subset of key proteins, i.e., Akt/ P-Akt, GSK/ P-GSK, MLP and melusin, involved in mechanosensing- and transduction and representing beneficial versus non-beneficial remodeling processes in animal models and humans with cardiac hypertrophy $[19,20,23,70,84,161,203]$. 

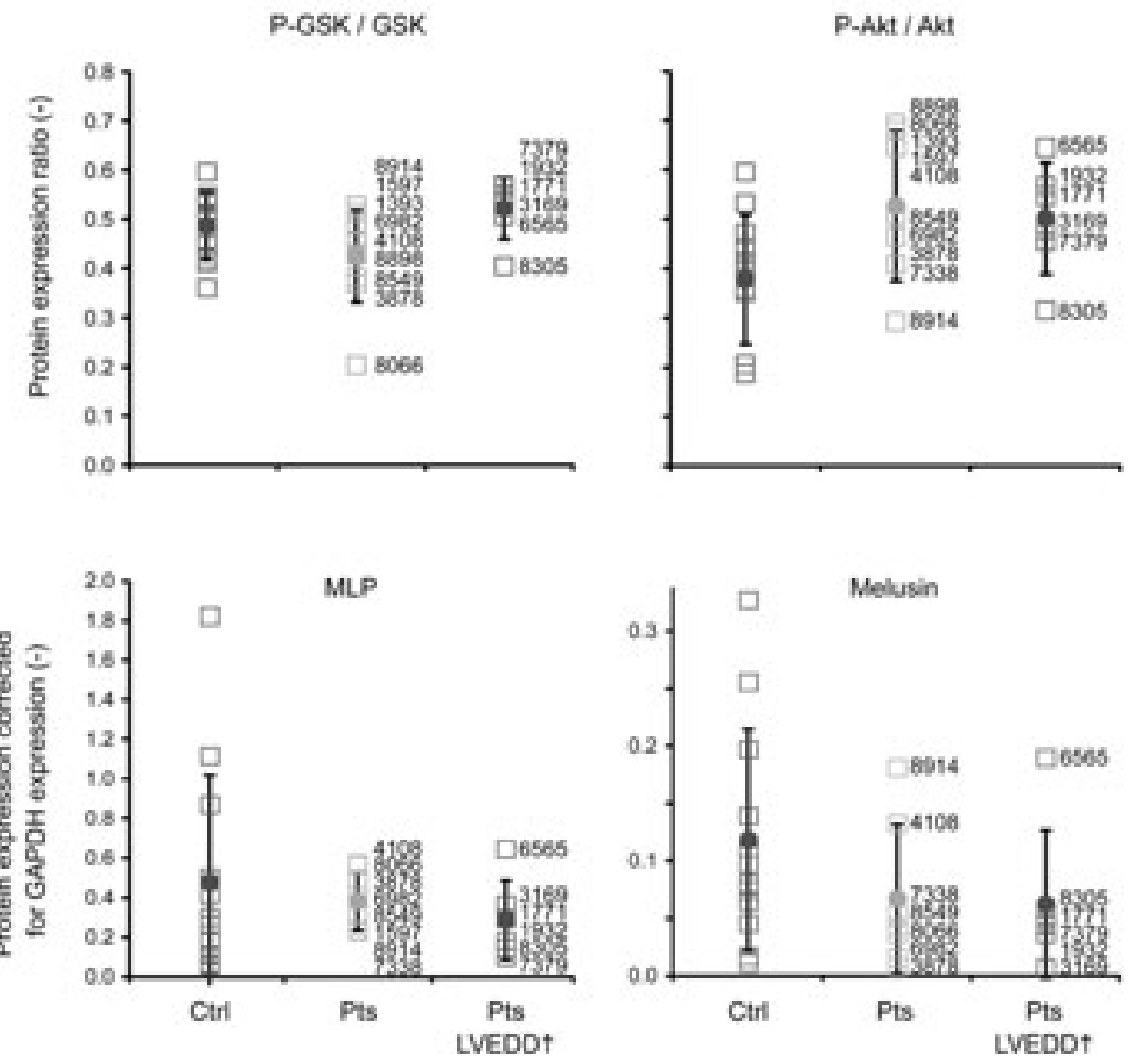

Figure $\mathbf{5}$ | Western blot analysis of key pro-compensatory proteins. Protein expression of Akt/ P-Akt (protein kinase B), glycogen synthase kinase 3B (GSK/ P-GSK), muscle LIM protein (MLP) and melusin. See text for details.

In a group comparison the expression of pro-compensatory key proteins was not significantly different from normal controls (Figure 5). A subgroup analysis in patients with an increased LV end-diastolic diameter, often considered an early manifestation of detrimental remodeling and imminent failure, also did not show a group mean protein expression pattern different from patients with a normal LV end-diastolic diameter or normal controls. These results are in line with others reports showing that patients with compensated or slightly decompensated ventricular hypertrophy might exhibit a relatively normal expression profile of these pro-compensatory proteins 
$[19,20,23,70,84,161,203]$. Additional studies in which the expression levels of the aforementioned proteins are investigated in patients with end-stage heart failure could be most relevant for comparative reasons.

In order to improve the clinical prediction of beneficial versus detrimental remodeling in individual patients, it may be helpful to integrate mechanical and myocardial phenotyping. Practically, patients with cardiac overload, e.g., due to valvular heart disease with an expected progression requiring surgical intervention within months, should be repeatedly characterized by CircAdapt modeling, especially when worsening of clinical symptoms occurs. In this way, serial analyses allow assessment of an individual load history. At the time of surgery, cardiac biopsies should be obtained and analyzed as described above, selecting key proteins that represent beneficial and detrimental aspects of remodeling. After surgery, when cardiac loading is expected to improve, mechanical assessment should be continued. This approach allows to integrate key markers of myocardial remodeling with long-term information on cardiac (over-)load. Moreover, if such an approach would demonstrate clear relations between cumulative mechanical load, i.e., the individual load history, and unfavorable myocardial remodeling, it could finally be concluded that biopsies are not needed to phenotype the myocardium, but serial mechanical phenotyping would be sufficient. In this context it should be mentioned that many markers of myocardial remodeling, other than those studied in this thesis, have been described as recently reviewed $[142,143]$. The choice of meaningful markers for individual patients will critically depend on 1) the specific form of cardiac disease, 2) the type of mechanical overload, 3) the duration of pathological stimuli imposed on the myocardium, 4) preexisting cardiac disease and relevant comorbidity and 5) the genotypic constitution of a patient. In the human patients analyzed in this thesis, clinical assessment did not suggest any known genetic defect, although this cannot be excluded. Nevertheless, it is clear that the individual cardiac phenotype is inseparably linked to the genotype and integrative clinical diagnostics should incorporate genetic testing when indicated.

The abovementioned considerations are still limiting the use of specific myocardial markers as valuable clinical tools to aid in patient-specific therapeutic decision making. Nevertheless, our results provide important insights into relevant myocardial remodeling processes present in cardiac overload. In order to make myocardial phenotyping practically applicable and clinically meaningful in patients with a cardiac mechanomyopathy, the selection of patients amenable for an integrative diagnostic workup should be well-defined. We propose that these patients should fulfill the following selection criteria, having a:

- High risk, suspicion or manifestation of cardiac disease, which likely results in life-threatening (mortality) or severely disabling sequelae (morbidity)

- Cardiac disease, which remains mechanistically largely unexplained, thereby preventing appropriate, preferentially causal therapy 
- Cardiac disease, which likely arises from multifactorial adverse myocardial remodeling, i.e., structural and functional, as an important consequence of past, present or expected future cardiac mechanical overload

In these selected patients, the applicable approaches could range from the use of molecular and cell-biological techniques used in myocardial tissue samples to clinically available non-invasive molecular imaging techniques $[142,143]$. In line with the data presented in Chapter 3 to Chapter 6 clear links between mechanical stimuli and alterations of myocardial structure and function could be established in individual patients by applying such an integrative diagnostic approach.

Altogether, the clinical assessment of selected patients with a cardiac mechanomyopathy should be adapted to incorporate mechanical, structural and functional cardiac analyses with emphasis on mechanistic characterization. This integrative approach of serial mechano-molecular phenotyping in patients should improve individualized diagnostic and therapeutic tailoring. In this sense the classic terminology discriminating pressure from volume overload becomes an outdated oversimplification. Cardiac mechanical overload and ventricular hypertrophy occurring in one individual patient are not comparable to those observed in another.

\section{CONCLUDING REMARKS}

In this thesis we sought to gain novel mechanistic insights into the role of distinct mechanical stimuli for molecular processes relevant for structural and functional remodeling during ventricular hypertrophy. We developed and explored an integrative approach that allows simultaneous assessment of mechanical overload and myocardial remodeling. We illustrate the opportunities and discuss the limitations of this mechano-structural-functional phenotyping in an animal model of overload-induced ventricular hypertrophy, accompanied by electrical and structural cardiomyocyte remodeling. Moreover, we applied mechano-structural-functional phenotyping to individual patients with mechanical overload and ventricular hypertrophy due to valvular heart disease undergoing cardiac surgery.

This thesis provides novel integrative concepts to recognize, diagnose, monitor and treat individual patients with a cardiac mechanomyopathy in a more mechanism-based way. 


\section{REFERENCES}

1. Adomian GE and Beazell J. Myofibrillar disarray produced in normal hearts by chronic electrical pacing. Am Heart J 112: 79-83, 1986.

2. Anderson RH, Ho SY, Redmann K, Sanchez-Quintana D, and Lunkenheimer PP. The anatomical arrangement of the myocardial cells making up the ventricular mass. Eur J Cardiothorac Surg 28: 517-525, 2005.

3. Arber S, Hunter JJ, Ross J, Jr., Hongo M, Sansig G, Borg J, Perriard JC, Chien KR, and Caroni P. MLPdeficient mice exhibit a disruption of cardiac cytoarchitectural organization, dilated cardiomyopathy, and heart failure. Cell 88: 393-403, 1997.

4. Armstrong PW, Stopps TP, Ford SE, and de Bold AJ. Rapid ventricular pacing in the dog: pathophysiologic studies of heart failure. Circulation 74: 1075-1084, 1986.

5. Arts T, Bovendeerd PH, Prinzen FW, and Reneman RS. Relation between left ventricular cavity pressure and volume and systolic fiber stress and strain in the wall. Biophys J 59: 93-102, 1991.

6. Arts T, Delhaas T, Bovendeerd P, Verbeek X, and Prinzen FW. Adaptation to mechanical load determines shape and properties of heart and circulation: the CircAdapt model. Am J Physiol Heart Circ Physiol 288 : H1943-1954, 2005.

7. Ashikaga $\mathrm{H}$, Coppola BA, Hopenfeld B, Leifer ES, McVeigh ER, and Omens JH. Transmural dispersion of myofiber mechanics: implications for electrical heterogeneity in vivo. J Am Coll Cardiol 49: 909-916, 2007.

8. Ashikaga $\mathrm{H}$, Covell JW, and Omens JH. Diastolic dysfunction in volume-overload hypertrophy is associated with abnormal shearing of myolaminar sheets. Am J Physiol Heart Circ Physiol 288: H2603-2610, 2005.

9. Ashikaga $\mathrm{H}, \mathrm{Omens} \mathrm{JH}$, and Covell JW. Time-dependent remodeling of transmural architecture underlying abnormal ventricular geometry in chronic volume overload heart failure. Am J Physiol Heart Circ Physiol 287: H1994-2002, 2004.

10. Baba HA, Stypmann J, Grabellus F, Kirchhof P, Sokoll A, Schafers M, Takeda A, Wilhelm MJ, Scheld HH, Takeda N, Breithardt G, and Levkau B. Dynamic regulation of MEK/Erks and Akt/GSK-3beta in human end-stage heart failure after left ventricular mechanical support: myocardial mechanotransductionsensitivity as a possible molecular mechanism. Cardiovasc Res 59: 390-399, 2003.

11. Barlucchi L, Leri A, Dostal DE, Fiordaliso F, Tada H, Hintze TH, Kajstura J, Nadal-Ginard B, and Anversa P. Canine ventricular myocytes possess a renin-angiotensin system that is upregulated with heart failure. Circ Res 88: 298-304, 2001.

12. Bausch AR, Ziemann F, Boulbitch AA, Jacobson $K$, and Sackmann E. Local measurements of viscoelastic parameters of adherent cell surfaces by magnetic bead microrheometry. Biophys J 75: 2038-2049, 1998.

13. Beazell JW, Adomian GE, Furmanski M, and Osborne SL. Complete atrioventricular block in dogs-compensation or decompensation. Am J Pathol 117: 154-157, 1984. 
14. Becker M, Franke A, Breithardt OA, Ocklenburg C, Kaminski T, Kramann R, Knackstedt C, Stellbrink C Hanrath $P$, Schauerte $P$, and Hoffmann R. Impact of left ventricular lead position on the efficacy of cardiac resynchronisation therapy: a two-dimensional strain echocardiography study. Heart 93: 11971203, 2007.

15. Becker M, Hoffmann R, Schmitz F, Hundemer A, Kuhl H, Schauerte $P$, Kelm M, and Franke A. Relation of optimal lead positioning as defined by three-dimensional echocardiography to long-term benefit of cardiac resynchronization. Am J Cardiol 100: 1671-1676, 2007.

16. Becker M, Kramann R, Franke A, Breithardt OA, Heussen N, Knackstedt C, Stellbrink C, Schauerte P, Kelm $\mathrm{M}$, and Hoffmann R. Impact of left ventricular lead position in cardiac resynchronization therapy on left ventricular remodelling. A circumferential strain analysis based on 2D echocardiography. Eur Heart $J 28$ : 1211-1220, 2007.

17. Ben-David J, Zipes DP, Ayers GM, and Pride HP. Canine left ventricular hypertrophy predisposes to ventricular tachycardia induction by phase 2 early afterdepolarizations after administration of BAY $\mathrm{K}$ 8644. J Am Coll Cardiol 20: 1576-1584, 1992.

18. Boriani G, Biffi M, Martignani C, Ziacchi M, Saporito D, Grigioni F, Domenichini G, Valzania C, Diemberger I, Bertini M, Specchia S, and Branzi A. Electrocardiographic remodeling during cardiac resynchronization therapy. Int J Cardiol 108: 165-170, 2006.

19. Brancaccio M, Fratta L, Notte A, Hirsch E, Poulet $R$, Guazzone $S$, De Acetis M, Vecchione $C$, Marino G, Altruda F, Silengo L, Tarone G, and Lembo G. Melusin, a muscle-specific integrin beta1-interacting protein, is required to prevent cardiac failure in response to chronic pressure overload. Nat Med 9: 68-75, 2003.

20. Brancaccio $M$, Hirsch $E$, Notte $A$, Selvetella G, Lembo G, and Tarone G. Integrin signalling: the tug-of-war in heart hypertrophy. Cardiovasc Res 70: 422-433, 2006.

21. Briguori C, Betocchi S, Manganelli F, Gigante B, Losi MA, Ciampi Q, Gottilla R, Violante A, Tocchetti CG, Volpe $M$, and Chiariello $M$. Determinants and clinical significance of natriuretic peptides and hypertrophic cardiomyopathy. Eur Heart J 22: 1328-1336, 2001.

22. Brockman SK. Cardiodynamics of Complete Heart Block. Am J Cardiol 16: 72-83, 1965.

23. Brokat $S$, Thomas J, Herda LR, Knosalla $C$, Pregla R, Brancaccio M, Accornero F, Tarone G, Hetzer R, and Regitz-Zagrosek V. Altered melusin expression in the hearts of aortic stenosis patients. Eur J Heart Fail 9: 568-573, 2007.

24. Buckberg GD, Clemente C, Cox JL, Coghlan HC, Castella M, Torrent-Guasp F, and Gharib M. The structure and function of the helical heart and its buttress wrapping. IV. Concepts of dynamic function from the normal macroscopic helical structure. Semin Thorac Cardiovasc Surg 13: 342-357, 2001.

25. Camm AJ, Janse MJ, Roden DM, Rosen MR, Cinca J, and Cobbe SM. Congenital and acquired long QT syndrome. Eur Heart J 21: 1232-1237, 2000.

26. Carabello BA, Mee R, Collins JJ, Jr., Kloner RA, Levin D, and Grossman W. Contractile function in chronic gradually developing subcoronary aortic stenosis. Am J Physiol 240: H80-84, 1981.

27. Carabello BA, Zile MR, Tanaka R, and Cooper Gt. Left ventricular hypertrophy due to volume overload versus pressure overload. Am J Physiol 263: H1137-1144, 1992. 
28. Cesselli D, Jakoniuk I, Barlucchi L, Beltrami AP, Hintze TH, Nadal-Ginard B, Kajstura J, Leri A, and Anversa P. Oxidative stress-mediated cardiac cell death is a major determinant of ventricular dysfunction and failure in dog dilated cardiomyopathy. Circ Res 89: 279-286, 2001.

29. Chaudhary KW, Rossman El, Piacentino V, 3rd, Kenessey A, Weber C, Gaughan JP, Ojamaa K, Klein I, Bers DM, Houser SR, and Margulies KB. Altered myocardial $\mathrm{Ca}^{2+}$ cycling after left ventricular assist device support in the failing human heart. J Am Coll Cardiol 44: 837-845, 2004.

30. Chen FY and Cohn LH. The surgical treatment of heart failure. A new frontier: nontransplant surgical alternatives in heart failure. Cardiol Rev 10: 326-333, 2002.

31. Coghlan HC, Coghlan AR, Buckberg GD, Gharib M, and Cox JL. The structure and function of the helical heart and its buttress wrapping. III. The electric spiral of the heart: The hypothesis of the anisotropic conducting matrix. Semin Thorac Cardiovasc Surg 13: 333-341, 2001.

32. Cohen P and Frame S. The renaissance of GSK3. Nat Rev Mol Cell Biol 2: 769-776, 2001.

33. Costa KD, Hunter PJ, Rogers JM, Guccione JM, Waldman LK, and McCulloch AD. A three-dimensional finite element method for large elastic deformations of ventricular myocardium: I--Cylindrical and spherical polar coordinates. J Biomech Eng 118: 452-463, 1996.

34. Costa KD, Hunter PJ, Wayne JS, Waldman LK, Guccione JM, and McCulloch AD. A three-dimensional finite element method for large elastic deformations of ventricular myocardium: Il--Prolate spheroidal coordinates. J Biomech Eng 118: 464-472, 1996.

35. Davlouros PA, Niwa K, Webb G, and Gatzoulis MA. The right ventricle in congenital heart disease. Heart 92 Suppl 1: i27-38, 2006.

36. De Acetis $M$, Notte $A$, Accornero $F$, Selvetella G, Brancaccio M, Vecchione $C$, Sbroggio M, Collino $F$, Pacchioni B, Lanfranchi G, Aretini A, Ferretti R, Maffei A, Altruda F, Silengo L, Tarone G, and Lembo G. Cardiac overexpression of melusin protects from dilated cardiomyopathy due to long-standing pressure overload. Circ Res 96: 1087-1094, 2005.

37. de Groot SH, Schoenmakers M, Molenschot MM, Leunissen JD, Wellens HJ, and Vos MA. Contractile adaptations preserving cardiac output predispose the hypertrophied canine heart to delayed afterdepolarization-dependent ventricular arrhythmias. Circulation 102: 2145-2151, 2000.

38. de Groot SH, Vos MA, Gorgels AP, Leunissen JD, van der Steld BJ, and Wellens HJ. Combining monophasic action potential recordings with pacing to demonstrate delayed afterdepolarizations and triggered arrhythmias in the intact heart. Value of diastolic slope. Circulation 92: 2697-2704, 1995.

39. de Tombe PP and ter Keurs HE. Sarcomere dynamics in cat cardiac trabeculae. Circ Res 68: 588-596, 1991.

40. Dell'Italia LJ, Meng QC, Balcells E, Straeter-Knowlen IM, Hankes GH, Dillon R, Cartee RE, Orr R, Bishop SP, Oparil S, and et al. Increased ACE and chymase-like activity in cardiac tissue of dogs with chronic mitral regurgitation. Am J Physiol 269: H2065-2073, 1995.

41. DiSalvo J, Reynolds R, Robinson JL, and Grupp G. Enhanced carotid sinus baroreflex responses in dogs with chronic A-V block. J Appl Physiol 38: 1-4, 1975.

42. Donker DW, Maessen JG, Spatjens RL, van der Nagel T, de Jong M, Ramaekers FC, Crijns HJ, Vos MA, and Volders PG. Serial left-ventricular biopsy sampling using a minimally invasive trans-thoracic approach in adult dogs. Pflugers Arch 454: 1043-1051, 2007. 
43. Donker DW, Maessen JG, Verheyen F, Ramaekers FC, Spatjens RL, Kuijpers H, Ramakers C, Schiffers PM, Vos MA, Crijns HJ, and Volders PG. Impact of acute and enduring volume overload on mechanotransduction and cytoskeletal integrity of canine left ventricular myocardium. Am J Physiol Heart Circ Physiol 292: H2324-2332, 2007.

44. Donker DW, Volders PG, Arts T, Bekkers BC, Hofstra L, Spatjens RL, Beekman JD, Borgers M, Crijns HJ, and Vos MA. End-diastolic myofiber stress and ejection strain increase with ventricular volume overload Serial in-vivo analyses in dogs with complete atrioventricular block. Basic Res Cardiol 100: 372-382, 2005.

45. Dorri F, Niederer PF, Redmann K, Lunkenheimer PP, Cryer CW, and Anderson RH. An analysis of the spatial arrangement of the myocardial aggregates making up the wall of the left ventricle. Eur J Cardiothorac Surg 31: 430-437, 2007.

46. Ecarnot-Laubriet A, De Luca K, Vandroux D, Moisant M, Bernard C, Assem M, Rochette L, and Teyssier JR. Downregulation and nuclear relocation of MLP during the progression of right ventricular hypertrophy induced by chronic pressure overload. J Mol Cell Cardiol 32: 2385-2395, 2000.

47. Emery $\mathrm{JL}$ and Omens JH. Mechanical regulation of myocardial growth during volume-overload hypertrophy in the rat. Am J Physiol 273: H1198-1204, 1997.

48. Emery JL, Omens JH, Mathieu-Costello OA, and McCulloch AD. Structural mechanisms of acute ventricular strain softening. Int J Cardiovasc Med Sci 1: 241-250, 1998.

49. Feldman RL, Nichols WW, Thompson LV, Paley DM, Goldman R, Conti CR, and Pepine CJ. Left ventricular changes resulting from chronic aortic regurgitation in dogs. Am J Cardiol 54: 890-892, 1984.

50. Flesch M, Margulies KB, Mochmann HC, Engel D, Sivasubramanian N, and Mann DL. Differential regulation of mitogen-activated protein kinases in the failing human heart in response to mechanical unloading. Circulation 104: 2273-2276, 2001.

51. Florenzano $\mathrm{F}$ and Glantz SA. Left ventricular mechanical adaptation to chronic aortic regurgitation in intact dogs. Am J Physiol 252: H969-984, 1987.

52. Frias PA, Corvera JS, Schmarkey L, Strieper M, Campbell RM, and Vinten-Johansen J. Evaluation of myocardial performance with conventional single-site ventricular pacing and biventricular pacing in a canine model of atrioventricular block. J Cardiovasc Electrophysiol 14: 996-1000, 2003.

53. Furukawa $T$, Ono $Y$, Tsuchiya $H$, Katayama $Y$, Bang ML, Labeit $D$, Labeit $S$, Inagaki $N$, and Gregorio CC. Specific interaction of the potassium channel beta-subunit minK with the sarcomeric protein T-cap suggests a T-tubule-myofibril linking system. J Mol Biol 313: 775-784, 2001.

54. Gaasch WH, Zile MR, Hoshino PK, Apstein CS, and Blaustein AS. Stress-shortening relations and myocardial blood flow in compensated and failing canine hearts with pressure-overload hypertrophy. Circulation 79: 872-883, 1989.

55. Gascho JA, Mueller TM, Eastham C, and Marcus ML. Effect of volume-overload hypertrophy on the coronary circulation awake dogs. Cardiovasc Res 16: 288-292, 1982.

56. Gaynor JW, Feneley MP, Gall SA, Jr., Savitt MA, Silvestry SC, Davis JW, Rankin JS, and Glower DD, Jr. Left ventricular adaptation to aortic regurgitation in conscious dogs. J Thorac Cardiovasc Surg 113: 149-158, 1997. 
57. Granzier H, Helmes M, Cazorla O, McNabb M, Labeit D, Wu Y, Yamasaki R, Redkar A, Kellermayer M, Labeit S, and Trombitas K. Mechanical properties of titin isoforms. Adv Exp Med Biol 481: 283-300; discussion 300-284, 2000.

58. Granzier $H$, Labeit $D, W u Y$, and Labeit $\mathrm{S}$. Titin as a modular spring: emerging mechanisms for elasticity control by titin in cardiac physiology and pathophysiology. J Muscle Res Cell Motil 23: 457-471, 2002.

59. Granzier H, Labeit D, Wu Y, Witt C, Watanabe K, Lahmers S, Gotthardt M, and Labeit S. Adaptations in titin's spring elements in normal and cardiomyopathic hearts. Adv Exp Med Biol 538: 517-530; discussion 530-511, 2003.

60. Granzier H and Labeit S. Cardiac titin: an adjustable multi-functional spring. J Physiol 541: 335-342, 2002.

61. Granzier $\mathrm{HL}$ and Labeit S. The giant muscle protein titin is an adjustable molecular spring. Exerc Sport Sci $\operatorname{Rev} 34:$ 50-53, 2006.

62. Granzier $\mathrm{HL}$ and Labeit S. The giant protein titin: a major player in myocardial mechanics, signaling, and disease. Circ Res 94: 284-295, 2004.

63. Granzier HL and Labeit S. Titin and its associated proteins: the third myofilament system of the sarcomere. Adv Protein Chem 71: 89-119, 2005.

64. Granzier HL, Wu Y, Trombitas K, Witt C, Labeit S, Bell S, and LeWinter M. Variable titin-based stiffness adjustment in heart disease. Circulation 108: e23, 2003.

65. Harding JD, Piacentino V, 3rd, Gaughan JP, Houser SR, and Margulies KB. Electrophysiological alterations after mechanical circulatory support in patients with advanced cardiac failure. Circulation 104: 12411247, 2001.

66. Hardt SE and Sadoshima J. Negative regulators of cardiac hypertrophy. Cardiovasc Res 63: 500-509, 2004.

67. Hasebe N, Hittinger L, Kohin S, Shen YT, Graham RM, and Vatner SF. Effects of hypertrophy on left atrial and ventricular compliance and plasma ANF levels in conscious dogs. Am J Physiol 268: H781-787, 1995.

68. Heineke J, Kempf T, Kraft T, Hilfiker A, Morawietz H, Scheubel RJ, Caroni P, Lohmann SM, Drexler H, and Wollert KC. Downregulation of cytoskeletal muscle LIM protein by nitric oxide: impact on cardiac myocyte hypertrophy. Circulation 107: 1424-1432, 2003.

69. Heineke J, Ruetten H, Willenbockel C, Gross SC, Naguib M, Schaefer A, Kempf T, Hilfiker-Kleiner D, Caroni P, Kraft T, Kaiser RA, Molkentin JD, Drexler H, and Wollert KC. Attenuation of cardiac remodeling after myocardial infarction by muscle LIM protein-calcineurin signaling at the sarcomeric Z-disc. Proc Natl Acad Sci U SA 102: 1655-1660, 2005.

70. Hirotani S, Zhai P, Tomita H, Galeotti J, Marquez JP, Gao S, Hong C, Yatani A, Avila J, and Sadoshima J. Inhibition of glycogen synthase kinase 3beta during heart failure is protective. Circ Res 101: 1164-1174, 2007.

71. Hittinger L, Patrick T, Ihara T, Hasebe N, Shen YT, Kalthof B, Shannon RP, and Vatner SF. Exercise induces cardiac dysfunction in both moderate, compensated and severe hypertrophy. Circulation 89: 2219-2231, 1994. 
72. Holmes JW. Candidate mechanical stimuli for hypertrophy during volume overload. J Appl Physiol 97: 1453-1460, 2004.

73. Hood WP, Jr., Rackley CE, and Rolett EL. Wall stress in the normal and hypertrophied human left ventricle. Am J Cardiol 22: 550-558, 1968.

74. Hunter PJ, Pullan AJ, and Smaill BH. Modeling total heart function. Annu Rev Biomed Eng 5: 147-177, 2003.

75. Iribe G, Helmes $M$, and Kohl P. Force-length relations in isolated intact cardiomyocytes subjected to dynamic changes in mechanical load. Am J Physiol Heart Circ Physiol 292: H1487-1497, 2007.

76. Ishihara K, Zile MR, Kanazawa S, Tsutsui H, Urabe Y, DeFreyte G, and Carabello BA. Left ventricular mechanics and myocyte function after correction of experimental chronic mitral regurgitation by combined mitral valve replacement and preservation of the native mitral valve apparatus. Circulation 86: Il16-25, 1992.

77. Januzzi JL, Jr., Peacock WF, Maisel AS, Chae CU, Jesse RL, Baggish AL, O'Donoghue M, Sakhuja R, Chen $A A$, van Kimmenade RR, Lewandrowski KB, Lloyd-Jones DM, and Wu AH. Measurement of the interleukin family member ST2 in patients with acute dyspnea: results from the PRIDE (Pro-Brain Natriuretic Peptide Investigation of Dyspnea in the Emergency Department) study. J Am Coll Cardiol 50: 607-613, 2007.

78. Januzzi JL, van Kimmenade R, Lainchbury J, Bayes-Genis A, Ordonez-Llanos J, Santalo-Bel M, Pinto YM, and Richards M. NT-proBNP testing for diagnosis and short-term prognosis in acute destabilized heart failure: an international pooled analysis of 1256 patients: the International Collaborative of NT-proBNP Study. Eur Heart J 27: 330-337, 2006.

79. Johannessen M, Moller S, Hansen T, Moens U, and Van Ghelue M. The multifunctional roles of the fourand-a-half-LIM only protein FHL2. Cell Mol Life Sci 63: 268-284, 2006.

80. Kadrmas JL and Beckerle MC. The LIM domain: from the cytoskeleton to the nucleus. Nat Rev Mol Cell Biol 5: 920-931, 2004.

81. Katayama K, Tajimi T, Guth BD, Matsuzaki M, Lee JD, Seitelberger R, and Peterson KL. Early diastolic filling dynamics during experimental mitral regurgitation in the conscious dog. Circulation 78: 390-400, 1988.

82. Kertesz NJ, Friedman RA, Colan SD, Walsh EP, Gajarski RJ, Gray PS, Shirley R, and Geva T. Left ventricular mechanics and geometry in patients with congenital complete atrioventricular block. Circulation 96 : 3430-3435, 1997.

83. Klotz S, Barbone A, Reiken S, Holmes JW, Naka Y, Oz MC, Marks AR, and Burkhoff D. Left ventricular assist device support normalizes left and right ventricular beta-adrenergic pathway properties. J Am Coll Cardiol 45: 668-676, 2005.

84. Knoll R, Hoshijima M, Hoffman HM, Person V, Lorenzen-Schmidt I, Bang ML, Hayashi T, Shiga N, Yasukawa H, Schaper W, McKenna W, Yokoyama M, Schork NJ, Omens JH, McCulloch AD, Kimura A, Gregorio CC, Poller W, Schaper J, Schultheiss HP, and Chien KR. The cardiac mechanical stretch sensor machinery involves a $Z$ disc complex that is defective in a subset of human dilated cardiomyopathy. Cell 111: 943-955, 2002.

85. Koide M, Hamawaki M, Narishige T, Sato H, Nemoto S, DeFreyte G, Zile MR, Cooper GI, and Carabello BA. Microtubule depolymerization normalizes in vivo myocardial contractile function in dogs with pressureoverload left ventricular hypertrophy. Circulation 102: 1045-1052, 2000. 
86. Kong Y, Shelton JM, Rothermel B, Li X, Richardson JA, Bassel-Duby R, and Williams RS. Cardiac-specific LIM protein FHL2 modifies the hypertrophic response to beta-adrenergic stimulation. Circulation 103: 2731-2738, 2001.

87. Koyanagi S, Eastham C, and Marcus ML. Effects of chronic hypertension and left ventricular hypertrophy on the incidence of sudden cardiac death after coronary artery occlusion in conscious dogs. Circulation 65: 1192-1197, 1982.

88. Krishnamurthy G, Patberg KW, Obreztchikova MN, Rybin AV, and Rosen MR. Developmental evolution of the delayed rectifier current IKs in canine heart appears dependent on the beta subunit minK. Heart Rhythm 1: 704-711, 2004.

89. Kupershmidt S, Yang IC, Sutherland M, Wells KS, Yang T, Yang P, Balser JR, and Roden DM. Cardiacenriched LIM domain protein fhl2 is required to generate I(Ks) in a heterologous system. Cardiovasc Res 56: 93-103, 2002.

90. Lammerding J, Schulze PC, Takahashi T, Kozlov S, Sullivan T, Kamm RD, Stewart CL, and Lee RT. Lamin A/C deficiency causes defective nuclear mechanics and mechanotransduction. J Clin Invest 113: 370-378, 2004.

91. Legault F, Rouleau JL, Juneau C, Rose C, and Rakusan K. Functional and morphological characteristics of compensated and decompensated cardiac hypertrophy in dogs with chronic infrarenal aorto-caval fistulas. Circ Res 66: 846-859, 1990.

92. Leri A, Barlucchi L, Limana F, Deptala A, Darzynkiewicz Z, Hintze TH, Kajstura J, Nadal-Ginard B, and Anversa P. Telomerase expression and activity are coupled with myocyte proliferation and preservation of telomeric length in the failing heart. Proc Natl Acad Sci U S A 98: 8626-8631, 2001.

93. Leshin SJ and Horwitz LD. A closed-chest catheter technique for producing aortic regurgitation in dogs. J Appl Physiol 33: 693-695, 1972.

94. LeWinter MM, Engler RL, and Karliner JS. Enhanced left ventricular shortening during chronic volume overload in conscious dogs. Am J Physiol 238: H126-133, 1980.

95. LeWinter MM, Wu Y, Labeit S, and Granzier H. Cardiac titin: structure, functions and role in disease. Clin Chim Acta 375: 1-9, 2007.

96. Li HY, Kotaka M, Kostin S, Lee SM, Kok LD, Chan KK, Tsui SK, Schaper J, Zimmermann R, Lee CY, Fung $\mathrm{KP}$, and Waye MM. Translocation of a human focal adhesion LIM-only protein, FHL2, during myofibrillogenesis and identification of LIM2 as the principal determinants of FHL2 focal adhesion localization. Cell Motil Cytoskeleton 48: 11-23, 2001.

97. Liu Z, Hilbelink DR, Crockett WB, and Gerdes AM. Regional changes in hemodynamics and cardiac myocyte size in rats with aortocaval fistulas. 1. Developing and established hypertrophy. Circ Res 69 : 52-58, 1991.

98. Liu Z, Hilbelink DR, and Gerdes AM. Regional changes in hemodynamics and cardiac myocyte size in rats with aortocaval fistulas. 2. Long-term effects. Circ Res 69: 59-65, 1991.

99. Lumens J, Delhaas T, Kirn B, and Arts T. Modeling ventricular interaction: a multiscale approach from sarcomere mechanics to cardiovascular system hemodynamics. Pac Symp Biocomput: 378-389, 2008.

100. Lunkenheimer PP, Redmann K, and Anderson RH. Further discursions concerning the unique myocardial band. Eur J Cardiothorac Surg 28: 779; author reply 780, 2005. 
101. Maeba T. Calcium-binding of cardiac sarcoplasmic reticulum and diastolic hemodynamics in volume overloaded canine hearts. Jpn Circ J 49: 163-170, 1985.

102. Marban E. Heart failure: the electrophysiologic connection. J Cardiovasc Electrophysiol 10: 1425-1428, 1999.

103. Mazhari R, Omens JH, Pavelec RS, Covell JW, and McCulloch AD. Transmural distribution of threedimensional systolic strains in stunned myocardium. Circulation 104: 336-341, 2001.

104. Meerson FZ. Compensatory hyperfunction of the heart and cardiac insufficiency. Circ Res 10: 250-258, 1962.

105. Moe GW, Stopps TP, Angus C, Forster C, De Bold AJ, and Armstrong PW. Alterations in serum sodium in relation to atrial natriuretic factor and other neuroendocrine variables in experimental pacing-induced heart failure. J Am Coll Cardiol 13: 173-179, 1989.

106. Molkentin JD, Lu JR, Antos CL, Markham B, Richardson J, Robbins J, Grant SR, and Olson EN. A calcineurindependent transcriptional pathway for cardiac hypertrophy. Cell 93: 215-228, 1998.

107. Morita S, Ochiai $Y$, Tanoue $Y$, Hisahara M, Masuda $M$, and Yasui H. Acute volume reduction with aortic valve replacement immediately improves ventricular mechanics in patients with aortic regurgitation. $J$ Thorac Cardiovasc Surg 125: 283-289, 2003.

108. Naito M, David D, Michelson EL, Schaffenburg M, and Dreifus LS. The hemodynamic consequences of cardiac arrhythmias: evaluation of the relative roles of abnormal atrioventricular sequencing, irregularity of ventricular rhythm and atrial fibrillation in a canine model. Am Heart J 106: 284-291, 1983.

109. Nattel S. Acquired delayed rectifier channelopathies: how heart disease and antiarrhythmic drugs mimic potentially-lethal congenital cardiac disorders. Cardiovasc Res 48: 188-190, 2000.

110. Newman WH. Contractile state of hypertrophied left ventricle in long-standing volume overload. Am J Physiol 234: H88-93, 1978.

111. Newman WH. Volume overload heart failure: length-tension curves, and response to beta-agonists, $\mathrm{Ca}^{2+}$, and glucagon. Am J Physiol 235: H690-700, 1978.

112. Nomura S. Diastolic property of left ventricle under experimental volume overload. Jpn Circ J 50: 426-432, 1986.

113. Omens JH. Stress and strain as regulators of myocardial growth. Prog Biophys Mol Biol 69: 559-572, 1998.

114. Omens JH and Covell JW. Transmural distribution of myocardial tissue growth induced by volumeoverload hypertrophy in the dog. Circulation 84: 1235-1245, 1991.

115. Pandalai PK, Bulcao CF, Merrill WH, and Akhter SA. Restoration of myocardial beta-adrenergic receptor signaling after left ventricular assist device support. J Thorac Cardiovasc Surg 131: 975-980, 2006.

116. Paulus WJ. Natriuretic peptides to probe haemodynamic overload in hypertrophic cardiomyopathy. Eur Heart J 22: 1249-1251, 2001.

117. Paulus WJ, Goethals MA, and Sys SU. Failure of myocardial inactivation: a clinical assessment in the hypertrophied heart. Basic Res Cardiol 87 Suppl 2: 145-161, 1992. 
118. Perreault CL, Shannon RP, Komamura K, Vatner SF, and Morgan JP. Abnormalities in intracellular calcium regulation and contractile function in myocardium from dogs with pacing-induced heart failure. $J$ Clin Invest 89: 932-938, 1992.

119. Perry GJ, Wei CC, Hankes GH, Dillon SR, Rynders P, Mukherjee R, Spinale FG, and Dell'Italia LJ. Angiotensin II receptor blockade does not improve left ventricular function and remodeling in subacute mitral regurgitation in the dog. J Am Coll Cardiol 39: 1374-1379, 2002.

120. Peschar M, Vernooy K, Cornelussen RN, Verbeek XA, Reneman RS, Vos MA, and Prinzen FW. Structural, electrical and mechanical remodeling of the canine heart in AV-block and LBBB. Eur Heart J 6: D61-D65, 2004.

121. Peschar M, Vernooy K, Vanagt WY, Reneman RS, Vos MA, and Prinzen FW. Absence of reverse electrical remodeling during regression of volume overload hypertrophy in canine ventricles. Cardiovasc Res 58 : 510-517, 2003.

122. Peterson KL, Ricci D, Tsuji J, Sasayama S, and Ross J, Jr. Evaluation of chamber and myocardial compliance in pressure overload hypertrophy. Eur J Cardiol 7 Suppl: 195-211, 1978.

123. Peterson KL, Tsuji J, Johnson A, DiDonna J, and LeWinter M. Diastolic left ventricular pressure-volume and stress-strain relations in patients with valvular aortic stenosis and left ventricular hypertrophy. Circulation 58: 77-89, 1978.

124. Prinzen FW, Augustijn CH, Arts T, Allessie MA, and Reneman RS. Redistribution of myocardial fiber strain and blood flow by asynchronous activation. Am J Physiol 259: H300-308, 1990.

125. Prinzen FW, Cheriex EC, Delhaas T, van Oosterhout MF, Arts T, Wellens HJ, and Reneman RS. Asymmetric thickness of the left ventricular wall resulting from asynchronous electric activation: a study in dogs with ventricular pacing and in patients with left bundle branch block. Am Heart J 130: 1045-1053, 1995.

126. Prinzen FW, Delhaas T, Arts T, and Reneman RS. Asymmetrical changes in ventricular wall mass by asynchronous electrical activation of the heart. Adv Exp Med Bio/ 346: 257-264, 1993.

127. Prinzen FW, Hunter WC, Wyman BT, and McVeigh ER. Mapping of regional myocardial strain and work during ventricular pacing: experimental study using magnetic resonance imaging tagging. J Am Coll Cardiol 33: 1735-1742, 1999.

128. Priori SG, Mantica M, and Schwartz PJ. Delayed afterdepolarizations elicited in vivo by left stellate ganglion stimulation. Circulation 78: 178-185, 1988.

129. Purcell NH, Darwis D, Bueno OF, Muller JM, Schule R, and Molkentin JD. Extracellular signal-regulated kinase 2 interacts with and is negatively regulated by the LIM-only protein FHL2 in cardiomyocytes. Mol Cell Biol 24: 1081-1095, 2004.

130. Razeghi P, Bruckner BA, Sharma S, Youker KA, Frazier $\mathrm{OH}$, and Taegtmeyer $\mathrm{H}$. Mechanical unloading of the failing human heart fails to activate the protein kinase B/Akt/glycogen synthase kinase-3beta survival pathway. Cardiology 100: 17-22, 2003.

131. Reynolds RD and DiSalvo J. Effects of dl-propranolol on atrial and ventricular rates in unanesthetized atrioventricular blocked dogs. J Pharmacol Exp Ther 205: 374-381, 1978.

132. Reynolds RD, Swan JF, and DiSalvo J. Relationship between cardiac hypertrophy and idioventricular rate in dogs with chronic A-V block. Life Sci 22: 1999-2002, 1978. 
133. Roden DM. Pharmacogenetics and drug-induced arrhythmias. Cardiovasc Res 50: 224-231, 2001.

134. Rosen MR. Is the response to programmed electrical stimulation diagnostic of mechanisms for arrhythmias? Circulation 73: II18-27, 1986.

135. Ross RS and Borg TK. Integrins and the myocardium. Circ Res 88: 1112-1119, 2001.

136. Sabbah HN, Goldberg AD, Schoels W, Kono T, Webb C, Brachmann J, and Goldstein S. Spontaneous and inducible ventricular arrhythmias in a canine model of chronic heart failure: relation to haemodynamics and sympathoadrenergic activation. Eur Heart J 13: 1562-1572, 1992.

137. Sabbah HN, Kono T, Stein PD, Mancini GB, and Goldstein S. Left ventricular shape changes during the course of evolving heart failure. Am J Physiol 263: H266-270, 1992.

138. Sabbah HN, Stein PD, Kono T, Gheorghiade M, Levine TB, Jafri S, Hawkins ET, and Goldstein S. A canine model of chronic heart failure produced by multiple sequential coronary microembolizations. Am $J$ Physiol 260: H1379-1384, 1991.

139. Sandler H and Dodge HT. Left Ventricular Tension and Stress in Man. Circ Res 13: 91-104, 1963.

140. Schoenmakers M, Ramakers C, van Opstal JM, Leunissen JD, Londono C, and Vos MA. Asynchronous development of electrical remodeling and cardiac hypertrophy in the complete AV block dog. Cardiovasc Res 59: 351-359, 2003.

141. Schreiner KD, Kelemen K, Zehelein J, Becker R, Senges JC, Bauer A, Voss F, Kraft P, Katus HA, and Schoels W. Biventricular hypertrophy in dogs with chronic AV block: effects of cyclosporin A on morphology and electrophysiology. Am J Physiol Heart Circ Physiol 287: H2891-2898, 2004.

142. Shirani J and Dilsizian V. Molecular imaging in heart failure. Curr Opin Biotechnol 18: 65-72, 2007.

143. Shirani J, Narula J, Eckelman WC, Narula N, and Dilsizian V. Early imaging in heart failure: exploring novel molecular targets. J Nucl Cardiol 14: 100-110, 2007.

144. Sipido KR, Bito V, Antoons G, Volders PG, and Vos MA. Na/Ca exchange and cardiac ventricular arrhythmias. Ann N Y Acad Sci 1099: 339-348, 2007.

145. Sipido KR, Volders PG, de Groot SH, Verdonck F, Van de Werf F, Wellens HJ, and Vos MA. Enhanced $\mathrm{Ca}(2+)$ release and $\mathrm{Na} / \mathrm{Ca}$ exchange activity in hypertrophied canine ventricular myocytes: potential link between contractile adaptation and arrhythmogenesis. Circulation 102: 2137-2144, 2000.

146. Sipido KR, Volders PG, Schoenmakers M, De Groot SH, Verdonck F, and Vos MA. Role of the $\mathrm{Na} / \mathrm{Ca}$ exchanger in arrhythmias in compensated hypertrophy. Ann N Y Acad Sci 976: 438-445, 2002.

147. Sipido KR, Volders PG, Vos MA, and Verdonck F. Altered $\mathrm{Na} / \mathrm{Ca}$ exchange activity in cardiac hypertrophy and heart failure: a new target for therapy? Cardiovasc Res 53: 782-805, 2002.

148. Spinale FG, Holzgrefe HH, Mukherjee R, Arthur SR, Child MJ, Powell JR, and Koster WH. LV and myocyte structure and function after early recovery from tachycardia-induced cardiomyopathy. Am J Physio/ 268: H836-847, 1995.

149. Spratt JA, Olsen CO, Tyson GS, Jr., Glower DD, Jr., Davis JW, and Rankin JS. Experimental mitral regurgitation. Physiological effects of correction on left ventricular dynamics. J Thorac Cardiovasc Surg 86: 479-489, 1983.

150. Starzl TE and Gaertner RA. Chronic heart block in dogs; a method for producing experimental heart failure. Circulation 12: 259-270, 1955. 
151. Starzl TE, Gaertner RA, and Baker RR. Acute complete heart block in dogs. Circulation 12: 82-89, 1955.

152. Stengl M, Ramakers C, Donker DW, Nabar A, Rybin AV, Spatjens RL, van der Nagel T, Wodzig WK, Sipido KR, Antoons G, Moorman AF, Vos MA, and Volders PG. Temporal patterns of electrical remodeling in canine ventricular hypertrophy: focus on IKs downregulation and blunted beta-adrenergic activation. Cardiovasc Res 72: 90-100, 2006.

153. Su-Fan Q, Brum JM, Kaye MP, and Bove AA. A new technique for producing pure aortic stenosis in animals. Am J Physiol 246: H296-301, 1984.

154. Su X, Wei CC, Machida N, Bishop SP, Hankes GH, Dillon RA, Oparil S, and Dell Italia LJ. Differential expression of angiotensin-converting enzyme and chymase in dogs with chronic mitral regurgitation. $J$ Mol Cell Cardiol 31: 1033-1045, 1999.

155. Sugiyama A, Ishida $Y$, Satoh $Y$, Aoki S, Hori M, Akie $Y$, Kobayashi $Y$, and Hashimoto K. Electrophysiological, anatomical and histological remodeling of the heart to AV block enhances susceptibility to arrhythmogenic effects of QT-prolonging drugs. Jpn J Pharmacol 88: 341-350, 2002.

156. Suto F, Zhu W, Cahill SA, Greenwald I, Navarro AL, and Gross GJ. Ventricular rate determines early bradycardic electrical remodeling. Heart Rhythm 2: 293-300, 2005.

157. Sweeney MO and Prinzen FW. A new paradigm for physiologic ventricular pacing. J Am Coll Cardiol 47: 282-288, 2006.

158. Tagawa H, Koide M, Sato $H$, Zile MR, Carabello BA, and Cooper Gt. Cytoskeletal role in the transition from compensated to decompensated hypertrophy during adult canine left ventricular pressure overloading. Circ Res 82: 751-761, 1998.

159. Takahara A, Sugiyama A, Satoh $Y$, Iwasaki H, Nakamura $Y$, and Hashimoto K. Cardiovascular profile of the canine torsades de pointes arrhythmia model assessed by echocardiographic and haemodynamic methods. Basic Clin Pharmacol Toxicol 101: 35-40, 2007.

160. Takeishi Y, Jalili T, Hoit BD, Kirkpatrick DL, Wagoner LE, Abraham WT, and Walsh RA. Alterations in $\mathrm{Ca}^{2+}$ cycling proteins and $G$ alpha $q$ signaling after left ventricular assist device support in failing human hearts. Cardiovasc Res 45: 883-888, 2000.

161. Tarone $G$ and Lembo G. Molecular interplay between mechanical and humoral signalling in cardiac hypertrophy. Trends Mol Med 9: 376-382, 2003.

162. ten Tusscher KH, Noble D, Noble PJ, and Panfilov AV. A model for human ventricular tissue. Am J Physiol Heart Circ Physiol 286: H1573-1589, 2004.

163. Tomaselli GF and Marban E. Electrophysiological remodeling in hypertrophy and heart failure. Cardiovasc Res 42: 270-283, 1999.

164. Torrent-Guasp F, Ballester M, Buckberg GD, Carreras F, Flotats A, Carrio I, Ferreira A, Samuels LE, and Narula J. Spatial orientation of the ventricular muscle band: physiologic contribution and surgical implications. J Thorac Cardiovasc Surg 122: 389-392, 2001.

165. Torrent-Guasp F, Buckberg GD, Clemente C, Cox JL, Coghlan HC, and Gharib M. The structure and function of the helical heart and its buttress wrapping. I. The normal macroscopic structure of the heart. Semin Thorac Cardiovasc Surg 13: 301-319, 2001. 
166. Trudel MC, Dube B, Potse M, Gulrajani RM, and Leon LJ. Simulation of ORST integral maps with a membrane-based computer heart model employing parallel processing. IEEE Trans Biomed Eng 51: 13191329, 2004.

167. Turina M, Bussmann WD, and Krayenbuhl HP. Contractility of the hypertrophied canine heart in chronic volume overload. Cardiovasc Res 3: 486-495, 1969.

168. Turitto $\mathrm{G}$ and El-Sherif N. Cardiac resynchronization therapy: a review of proarrhythmic and antiarrhythmic mechanisms. Pacing Clin Electrophysiol 30: 115-122, 2007.

169. Urabe Y, Mann DL, Kent RL, Nakano K, Tomanek RJ, Carabello BA, and Cooper Gt. Cellular and ventricular contractile dysfunction in experimental canine mitral regurgitation. Circ Res 70: 131-147, 1992.

170. Urschel CW, Covell JW, Sonnenblick EH, Ross J, Jr., and Braunwald E. Myocardial mechanics in aortic and mitral valvular regurgitation: the concept of instantaneous impedance as a determinant of the performance of the intact heart. J Clin Invest 47: 867-883, 1968.

171. Van Der Toorn A, Barenbrug P, Snoep G, Van Der Veen FH, Delhaas T, Prinzen FW, Maessen J, and Arts T. Transmural gradients of cardiac myofiber shortening in aortic valve stenosis patients using MRI tagging. Am J Physiol Heart Circ Physiol 283: H1609-1615, 2002.

172. van Kimmenade RR, Januzzi JL, Jr., Ellinor PT, Sharma UC, Bakker JA, Low AF, Martinez A, Crijns HJ, MacRae CA, Menheere PP, and Pinto YM. Utility of amino-terminal pro-brain natriuretic peptide, galectin-3, and apelin for the evaluation of patients with acute heart failure. J Am Coll Cardiol 48: 1217-1224, 2006.

173. van Kimmenade RR, Pinto YM, Bayes-Genis A, Lainchbury JG, Richards AM, and Januzzi JL, Jr. Usefulness of intermediate amino-terminal pro-brain natriuretic peptide concentrations for diagnosis and prognosis of acute heart failure. Am J Cardiol 98: 386-390, 2006.

174. van Oosterhout MF, Prinzen FW, Arts T, Schreuder JJ, Vanagt WY, Cleutjens JP, and Reneman RS. Asynchronous electrical activation induces asymmetrical hypertrophy of the left ventricular wall. Circulation 98: 588-595, 1998.

175. van Opstal JM, Verduyn SC, Leunissen HD, de Groot SH, Wellens HJ, and Vos MA. Electrophysiological parameters indicative of sudden cardiac death in the dog with chronic complete AV-block. Cardiovasc Res 50: 354-361, 2001.

176. Vanagt WY, Prinzen FW, and Delhaas T. Reversal of pacing-induced heart failure by left ventricular apical pacing. N Engl J Med 357: 2637-2638, 2007.

177. Verdonck F, Volders PG, Vos MA, and Sipido KR. Increased $\mathrm{Na}^{+}$concentration and altered $\mathrm{Na} / \mathrm{K}$ pump activity in hypertrophied canine ventricular cells. Cardiovasc Res 57: 1035-1043, 2003.

178. Verduyn SC, Ramakers C, Snoep G, Leunissen JD, Wellens HJ, and Vos MA. Time course of structural adaptations in chronic AV block dogs: evidence for differential ventricular remodeling. Am J Physiol Heart Circ Physiol 280: H2882-2890, 2001.

179. Vernooy K, Cornelussen RN, Verbeek XA, Vanagt WY, van Hunnik A, Kuiper M, Arts T, Crijns HJ, and Prinzen FW. Cardiac resynchronization therapy cures dyssynchronopathy in canine left bundle-branch block hearts. Eur Heart J 28: 2148-2155, 2007.

180. Vernooy K, Dijkman B, Cheriex EC, Prinzen FW, and Crijns HJ. Ventricular remodeling during long-term right ventricular pacing following His bundle ablation. Am J Cardiol 97: 1223-1227, 2006. 
181. Vernooy K, Verbeek XA, Cornelussen RN, Dijkman B, Crijns HJ, Arts T, and Prinzen FW. Calculation of effective VV interval facilitates optimization of AV delay and VV interval in cardiac resynchronization therapy. Heart Rhythm 4: 75-82, 2007.

182. Vernooy K, Verbeek XA, Peschar M, Crijns HJ, Arts T, Cornelussen RN, and Prinzen FW. Left bundle branch block induces ventricular remodelling and functional septal hypoperfusion. Eur Heart J 26: 91-98, 2005.

183. Vernooy K, Verbeek XA, Peschar M, and Prinzen FW. Relation between abnormal ventricular impulse conduction and heart failure. J Interv Cardiol 16: 557-562, 2003.

184. Villarreal D, Freeman $\mathrm{RH}$, and Johnson RA. Neurohumoral modulators and sodium balance in experimental heart failure. Am J Physiol 264: H1187-1193, 1993.

185. Volders PG, Sipido KR, Vos MA, Kulcsar A, Verduyn SC, and Wellens HJ. Cellular basis of biventricular hypertrophy and arrhythmogenesis in dogs with chronic complete atrioventricular block and acquired torsade de pointes. Circulation 98: 1136-1147, 1998.

186. Volders PG, Sipido KR, Vos MA, Spatjens RL, Leunissen JD, Carmeliet E, and Wellens HJ. Downregulation of delayed rectifier $\mathrm{K}(+)$ currents in dogs with chronic complete atrioventricular block and acquired torsades de pointes. Circulation 100: 2455-2461, 1999.

187. Volders PG, Vos MA, Szabo B, Sipido KR, de Groot SH, Gorgels AP, Wellens HJ, and Lazzara R. Progress in the understanding of cardiac early afterdepolarizations and torsades de pointes: time to revise current concepts. Cardiovasc Res 46: 376-392, 2000.

188. Vos MA, de Groot SH, Verduyn SC, van der Zande J, Leunissen HD, Cleutjens JP, van Bilsen M, Daemen MJ, Schreuder JJ, Allessie MA, and Wellens HJ. Enhanced susceptibility for acquired torsade de pointes arrhythmias in the dog with chronic, complete AV block is related to cardiac hypertrophy and electrical remodeling. Circulation 98: 1125-1135, 1998.

189. Vos MA, van Opstal JM, Leunissen JD, and Verduyn SC. Electrophysiologic parameters and predisposing factors in the generation of drug-induced Torsade de Pointes arrhythmias. Pharmacol Ther 92: 109-122, 2001.

190. Voss F, Schreiner KD, Senges JC, Becker R, Bauer A, Kuebler W, and Schoels W. Refractory patterns and susceptibility to drug-induced polymorphic ventricular tachycardias in dogs with chronic atrioventricular block: relation to the type of anesthesia. J Cardiovasc Pharmacol 40: 651-659, 2002.

191. Webb $\mathrm{G}$ and Gatzoulis MA. Atrial septal defects in the adult: recent progress and overview. Circulation 114: 1645-1653, 2006.

192. Weber KT, Pick R, Silver MA, Moe GW, Janicki JS, Zucker IH, and Armstrong PW. Fibrillar collagen and remodeling of dilated canine left ventricle. Circulation 82: 1387-1401, 1990.

193. Wei CM, Heublein DM, Perrella MA, Lerman A, Rodeheffer RJ, McGregor CG, Edwards WD, Schaff HV, and Burnett JC, Jr. Natriuretic peptide system in human heart failure. Circulation 88: 1004-1009, 1993.

194. Weinberg EO, Shimpo M, Hurwitz S, Tominaga S, Rouleau JL, and Lee RT. Identification of serum soluble ST2 receptor as a novel heart failure biomarker. Circulation 107: 721-726, 2003.

195. Williams GJ, Elston J, and Muir JR. A simplified technique for the production of experimental aortic regurgitation. Cardiovasc Res 9: 440-442, 1975. 
196. Winckels SK, Thomsen MB, Oosterhoff P, Oros A, Beekman JD, Attevelt NJ, Kretzers L, and Vos MA. Highseptal pacing reduces ventricular electrical remodeling and proarrhythmia in chronic atrioventricular block dogs. J Am Coll Cardiol 50: 906-913, 2007.

197. Winkler B, Schaper J, and Thiedemann KU. Hypertrophy due to chronic volume overloading in the dog heart. A morphometric study. Basic Res Cardiol 72: 222-227, 1977.

198. Wusten B, Flameng $W$, and Schaper W. Cardiac function in the chronically volume-overloaded canine heart. Basic Res Cardiol 72: 172-177, 1977.

199. Wusten B, Flameng W, Winkler B, and Schaper W. Role of cardiac contractility in hypertrophy from chronic volume loading. Cardiovasc Res 11: 132-140, 1977.

200. Yin FC. Ventricular wall stress. Circ Res 49: 829-842, 1981.

201. Yun KL, Rayhill SC, Niczporuk MA, Fann JI, Derby GC, Daughters GT, Ingels NB, Jr., and Miller DC. Left ventricular mechanics and energetics in the dilated canine heart: acute versus chronic mitral regurgitation. J Thorac Cardiovasc Surg 104: 26-39, 1992.

202. Zipes DP and Wellens HJ. Sudden cardiac death. Circulation 98: 2334-2351, 1998.

203. Zolk O, Caroni P, and Bohm M. Decreased expression of the cardiac LIM domain protein MLP in chronic human heart failure. Circulation 101: 2674-2677, 2000. 


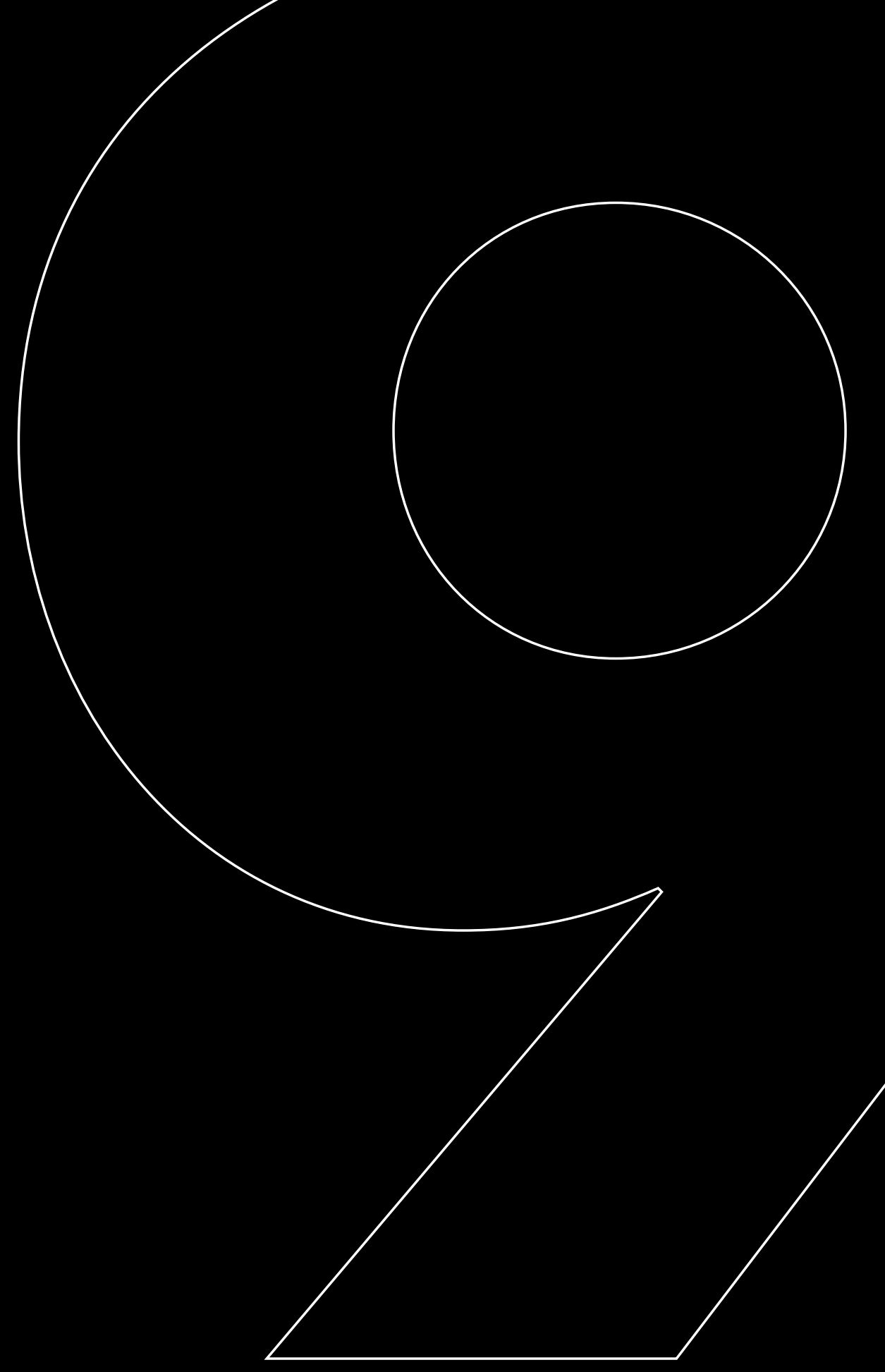





\title{
Summary
}

\section{Samenvatting}

Zusammenfassung

\author{
Appendix \\ Full colour illustrations
}

Dankwoord

Publications 


\section{SUMMARY}

In Chapter 1, we explain the meaning of "cardiac mechanomyopathy". We illustrate current inabilities to carefully identify mechanical stimuli influencing the heart under normal conditions and in hemodynamic overload. Also, it is realized that molecular pathways of mechanotransduction, hypertrophy and mechanoelectrical coupling are often poorly resolved in individual patients with cardiac overload. Anno 2008, the gap between cardiac genotype and phenotype in mechanomyopathy is still very large.

In Chapter 2, we address experimental and clinical evidence that mechanical forces are important stimuli for structural and molecular-electrical alterations during cardiac overload, which can finally set the stage for potentially life-threatening arrhythmias. Yet, the mechanisms underlying the mutual interaction between mechanics, structural and electrical remodeling remain to be elucidated. Recent reports suggest that cardiomyocytes are able to sense and transduce mechanical forces through specific cardiomyocyte Z-disk protein complexes. Moreover, physical connections between these protein complexes and ion channels have been suggested. These findings support the notion that mechano-structural links may play a role in mediating overload-induced, i.e., acquired, ion-channelopathies.

To investigate mechanotransduction in cardiac overload, we selected the canine model of complete atrioventricular block (AVB) with pronounced ventricular (and atrial) proarrhythmia. In this model of bradycardia-induced cardiac overload the arrhythmogenic role of impaired repolarization has been established, being mainly mediated by $\mathrm{K}^{+}$-channel downregulation and altered cellular $\mathrm{Ca}^{2+}$ handling.

As a prerequisite for further studies on mechanotransduction, we have characterized in Chapter $\mathbf{3}$ the left ventricular (LV) myocardial mechanics acting in the AVB model. In serial acute-to-chronic experiments in the intact beating heart, we found that end-diastolic stress and strain as well as ejection strain, but not systolic stress, are significantly increased. These early increases, i.e., acute to 2 weeks $A V B$, coincided with peak hypertrophic growth, supporting the role of stress and strain as primary stimuli for mechanotransduction. Our approach, using echocardiography and invasive blood pressure measurements combined with mathematical modeling of mechanics, provided values of stress and strain which were similar to data obtained by others in experiments on isolated cardiac muscle. In this way, accurate quantification of myocardial mechanics in vivo allows extrapolation of data from and to in-vitro experiments, enabling comprehensive assessment of mechanotransduction.

In order to combine serial mechanical phenotyping described in Chapter $\mathbf{3}$ with a long-term analysis of myocardial tissue remodeling, we developed a minimally-invasive transthoracic approach to obtain serial LV intramural biopsies, as described in Chapter 4. This technique enables repetitive sampling in the same individuals yielding relatively large myocardial biopsies as compared to commonly-used catheter-based techniques. The tissue specimens were found to be of excellent quality and suitable for the use of various cell-biological and molecular analyses. Moreover, after 
slight adaptations the technique was found to be safe, did not influence functional and structural characteristics of the AVB heart and could be extended to other animal models.

In Chapter 5, we applied the novel biopsy technique described in Chapter 4 to study time-related aspects of electrical remodeling in the AVB model. The impetus to this study was the general lack of temporal information on the electromolecular alterations underlying ion-channel downregulation in this and other arrhythmogenic models. We examined the acute-to-chronic time course of downregulation of the subunits KCNO1 and KCNE1 of the slowly-activating delayed rectifier $\left(\mathrm{I}_{\mathrm{KS}}\right)$ channel and its $\beta$-adrenergic repolarization responsiveness. In serial apicolateral LV biopsies, mRNA and protein levels of the $I_{\mathrm{Ks}}$ pore-forming subunit KCNO1 were significantly decreased within a few days after induction of AVB. This downregulation was followed by a gradual decrease of $\beta 1$-adrenergic receptor mRNA and protein expression. In the same time interval, plasma levels of norepinephrine were found to be transiently elevated. Functional studies incorporating $\beta$-adrenergic provocation testing in vivo revealed repolarization instability, i.e., loss of repolarization shortening and increased beat-to-beat variability of repolarization. This was found to be at least partly based on a decreased $\beta$-adrenergic activation of $\mathrm{I}_{\mathrm{Ks}}$. These changes favor the occurrence of ventricular arrhythmias in this acquired form of ion-channelopathy.

As a next step, in Chapter 6, we focussed on the mechanisms of mechanotransduction in the AVB model by assessing ventricular mechanics (Chapter 3 ) and the expression of myocardial key proteins involved in mechanosensing- and transduction over time. During early increases of mechanical load, reflected by end-diastolic stress, ejection strain and brain natriuretic peptide (BNP) release after induction of $A V B$, we found a transient compromise of cytoskeletal integrity, based at least partly on transcriptional downregulation. A more advanced disruption of the myofibrillar apparatus and the desmin network did not occur and a cytoskeletal reorganization ensued, which coincided with the upregulation of pro-compensatory proteins such as melusin, P-Akt (protein kinase B), P-glycogen synthase kinase (GSK) and muscle LIM protein (MLP), indicating a strong drive towards compensated hypertrophy.

In order to translate our findings to human patients suffering from cardiac overload, we describe a novel approach in Chapter 7, which ultimately allows to quantify the mechanical forces relevant for myocardial remodeling, i.e., stress and strain, at the bedside. We report our first experiences with this completely non-invasive modality to quantify ventricular hemodynamics and mechanics in patients with different forms of cardiac overload. Using the CircAdapt computer model (developed at the Department of Biophysics, Cardiovascular Research Institute Maastricht) in combination with transthoracic echocardiography and armcuff blood pressure measurements, we found a high degree of accuracy of the CircAdapt simulation in comparison with invasive measurements of LV hemodynamics. Thus, accurate patient-specific modeling of LV intracardiac hemodynamics and myofiber mechanics is practically feasible by completely non-invasive means. The approach has important clinical potential as a comprehensive bedside assessment of systolic and diastolic cardiac function and loading conditions, i.e., pressure-volume and stress-strain relations, considered as triggers of cardiac remodeling and indicators of prognosis. 


\section{SAMENVATTING}

In Hoofdstuk 1 wordt de betekenis van het begrip "cardiale mechanomyopathie" verklaard. Anno 2008 is het nog steeds zeer moeilijk, zo niet onmogelijk, om de mechanische stimuli die het hart onder normale omstandigheden en bij hemodynamische overbelasting beïnvloeden, nauwkeurig te identificeren. Bovendien zijn de moleculaire processen van mechanotransductie, hypertrofie en mechano-elektrische koppeling zeer beperkt opgehelderd. De kloof tussen cardiaal genotype en fenotype bij mechanomyopathie blijkt nog steeds immens.

In Hoofdstuk 2 wordt beschreven (op basis van experimentele en klinische onderzoeksresultaten) dat mechanische krachten belangrijke stimuli vormen voor structurele en moleculair-elektrische veranderingen tijdens cardiale overbelasting. Deze veranderingen kunnen uiteindelijk het substraat vormen voor het optreden van (in potentie) levensbedreigende kamerritmestoornissen. De mechanismen die ten grondslag liggen aan de interactie tussen mechanica enerzijds en structurele en elektrische remodelering anderzijds, worden belicht. Uit recente studies komt naar voren dat cardiomyocyten in staat zijn om mechanische krachten te detecteren en over te brengen via cardiomyocyt-specifieke Z-disk eiwitcomplexen. Bovendien is aangetoond dat deze eiwitcomplexen in direct contact staan met ionkanalen. Deze bevindingen ondersteunen het idee dat mechanostructurele verbanden in de myocyt een rol spelen bij het ontstaan van door overbelasting geïnduceerde ionkanaalpathologie. Om mechanotransductie bij cardiale overbelasting te onderzoeken, is een hondenmodel gekozen met compleet atrioventriculair blok (AVB) en uitgesproken ventriculaire (en atriale) pro-aritmie. In dit model, met bradycardie-geïnduceerde cardiale overbelasting, is de aritmogene rol van een gestoorde repolarisatie van de hartkamers onmiskenbaar vast komen te staan. Deze wordt hoofdzakelijk veroorzaakt door downregulatie van kaliumkanalen en een veranderde calciumhuishouding in de cel.

Alvorens over te gaan tot verder onderzoek naar mechanotransductie worden in Hoofdstuk $\mathbf{3}$ de mechanische veranderingen in hartspierweefsel van de linkerventrikel beschreven die een rol spelen in het AVB model. In seriële acuut-chronische experimenten in het intacte kloppende hart werd gevonden dat eind-diastolische stress en strain, evenals ejectie-strain, significant verhoogd zijn. Systolische stress blijkt niet verhoogd te zijn. Deze vroege toename van mechanische krachten, vanaf het acute moment tot ongeveer 2 weken na AVB, gaat gepaard met een piek in de toename van hypertrofie. Dit ondersteunt de veronderstelling dat stress en strain een belangrijke rol spelen als primaire stimuli voor mechanotransductie. Onze benadering, waarbij we gebruik maken van echocardiografie en invasieve bloeddrukmetingen in combinatie met wiskundige modellen van cardiale mechanica, levert waarden op van strain en stress die vergelijkbaar zijn met die verkregen tijdens experimenten in geïsoleerd hartspierweefsel. Op deze manier is het extrapoleren van in-vivo data naar in-vitro resultaten, en vice versa, mogelijk en behoren translationele bepalingen van mechanotransductie tot de mogelijkheden.

In Hoofdstuk 4 wordt een nieuwe minimaal-invasieve transthoracale methode beschreven waarmee myocardbiopten uit de linkerventrikelwand serieel kunnen worden verkregen. Daardoor kan de 
moleculaire analyse van myocardiale remodelering worden gerelateerd aan seriële mechanische fenotyperingen, zoals ook beschreven in Hoofdstuk 4. Deze methode maakt het mogelijk om bij dezelfde individuen herhaalde biopten te nemen, die in vergelijking met eerdere methoden relatief groot zijn. De weefsels die bij biopsie werden verkregen blijken van een uitstekende kwaliteit te zijn en geschikt voor diverse celbiologische, moleculaire en electrofysiologische bepalingen. Verder blijkt deze bioptmethode na geringe aanpassingen veilig en worden de functionele en structurele kenmerken van het AVB-hart niet aangetast. De methode kan ook bij andere diermodellen worden toegepast.

In Hoofdstuk 5 worden tijdsafhankelijke aspecten van elektrische remodelering in het AVB model beschreven. De reden van dit onderzoek was het algemene gebrek aan temporele informatie over elektromoleculaire veranderingen in dit en andere modellen van ritmestoornissen. We onderzochten het beloop van de afname van de subunits KCNQ1 en KCNE1 van het $I_{\mathrm{Ks}}$ ionkanaal (slowly-activating delayed rectifier $\mathrm{K}^{+}$current) en de $\beta$-adrenerge gevoeligheid daarvan. In seriële biopten uit de apicolaterale linkerventrikel bleken mRNA en hoeveelheid eiwit van de kanaalsubunit KCNQ1 significant verlaagd binnen een paar dagen na inductie van AVB. Hierna volgde een geleidelijke afname van mRNA en eiwitexpressie van de $\beta 1$-adrenerge receptor. In hetzelfde tijdsinterval werden in het bloedplasma tijdelijk verhoogde concentraties noradrenaline gevonden. In-vivo studies tonen instabiliteit van de repolarisatie aan, te weten het verlies aan vermogen van de repolarisatie om te verkorten tijdens $\beta$-adrenerge stimulatie, alsmede een toename van de slag-op-slag variabiliteit van repolarisatieduur. Dit fenomeen blijkt ten dele gebaseerd te zijn op een afgenomen $\beta$-adrenerge activatie van $I_{\mathrm{Ks}}$; op deze manier wordt het optreden van ventriculaire aritmieën bij deze verworven vorm van ionkanaalpathologie bevorderd.

In Hoofdstuk 6 wordt aandacht besteed aan de mechanismen van mechanotransductie in het AVB model door middel van het bepalen van ventriculaire mechanica (Hoofdstuk 3) en de expressie van myocardiale sleuteleiwitten die betrokken zijn bij mechanosensing en -transductie. Gedurende de vroege toename van mechanische belasting, die gekenmerkt wordt door toegenomen einddiastolische stress, ejectie-strain en het vrijkomen van brain natriuretic peptide (BNP), zien wij een tijdelijk gecompromitteerde integriteit van het cytoskelet, die op zijn minst gedeeltelijk gebaseerd is op downregulatie van de transcriptie van bepaalde eiwitten. Een verdere verstoring van het myofibrillaire apparaat en het desmine-netwerk treedt niet op, maar er vindt wel een reorganisatie van het cytoskelet plaats, die gepaard gaat met een toename van compenserende eiwitten zoals melusine, P-Akt (proteine kinase B), P-glycogeen synthase kinase (GSK) en spier LIM proteine (MLP). Deze veranderingen duiden een sterke neiging tot gecompenseerde hypertrofie aan.

Om de nieuwe bevindingen te kunnen vertalen naar patiënten, die lijden aan cardiale overbelasting, beschrijven we in Hoofdstuk 7 een benadering die ons in staat stelt om de mechanische krachten, relevant voor myocardiale remodeling, te kwantificeren aan het bed van de patiënt. We rapporteren de eerste ervaringen met dit volledig niet-invasieve model om ventriculaire hemodynamiek en mechanica bij patiënten met verschillende vormen van cardiale overbelasting in getal uit te drukken. Door gebruikmaking van het CircAdapt computer model (ontwikkeld binnen de afdeling 
Biofysica van het Cardiovasculair Onderzoeksinstituut CARIM te Maastricht) in combinatie met transthoracale echocardiografie en niet-invasieve bloeddrukmeting, kon worden vastgesteld dat de CircAdapt simulatie de hemodynamiek van de linkerventrikel op een zeer nauwkeurige wijze weergeeft, te vergelijken met meetresultaten die worden verkregen tijdens invasieve procedures.

Concluderend is het in de praktijk haalbaar om op een volledig niet-invasieve manier patiëntspecifieke intracardiale hemodynamiek en hartspiervezel-mechanica te modelleren. De benadering verschaft belangrijke klinische mogelijkheden om aan het bed van de patiënt de systolische en diastolische parameters, die betrokken zijn bij de belasting van het hart, te bepalen. Het gaat hier met name om druk-volume en stress-strain relaties, die beschouwd worden als triggers voor cardiale remodelering en prognostische factoren. 


\section{ZUSAMMENFASSUNG}

In Kapitel 1 wird die Bedeutung des Begriffs "Kardiale Mechanomyopathie" erläutert. Anno 2008 ist es noch immer schwierig, wenn nicht sogar unmöglich, die mechanischen Faktoren, die das Herz unter normalen Bedingungen aber auch bei hämodynamischer Überbelastung beeinflussen, auf eine exakte Art und Weise zu identifizieren. Im Übrigen sind die molekularen Prozesse der Mechanotransduktion, der Hypertrophie und der mechano-elektrischen Kopplung nur in begrenztem Maße entschlüsselt. In diesem Sinne zeigen sich bei der Mechanomyopathie auch noch große Lücken zum Verständnis der Zusammenhänge zwischen Genotyp und Phänotyp.

In Kapitel 2 wird beschrieben (basierend auf experimentellen und klinischen Forschungsergebnissen), dass mechanische Kräfte wichtige Reize für strukturelle und molekular-elektrische Veränderungen im Rahmen einer kardialen Überbelastung darstellen. Diese Veränderungen können letztendlich ein Substrat für das Auftreten von (potentiell) lebensbedrohlichen ventrikulären Herzrhythmusstörungen bilden. Die Mechanismen, die die Grundlage der Interaktion zwischen den mechanischen Kräften einerseits und den strukturellen und elektrischen Remodellierungsprozessen andererseits bilden, werden erläutert. In aktuellen Studien wird berichtet, dass Kardiomyozyten über spezifische sogenannte Z-Scheiben-Eiweißkomplexe in der Lage sind, mechanische Kräfte zu detektieren und weiterzuleiten. Es konnte ferner gezeigt werden, dass diese Eiweißkomplexe in direktem Kontakt zu lonenkanälen stehen. Diese Ergebnisse stützen die Hypothese, dass mechanostrukturelle Interaktionen in Kardiomyozyten beim Entstehen der durch Überbelastung induzierten Ionenkanalpathologie eine Rolle spielen. Um die Mechanotransduktion bei kardialer Überbelastung zu analysieren, wurde ein Hundemodell mit komplettem atrio-ventrikulären Block (AVB) und deutlicher ventrikulärer (und atrialer) Pro-Arrhythmie untersucht. In diesem Modell mit Bradykardieinduzierter kardialer Überbelastung steht die arrhythmogene Rolle der gestörten Repolarisation der Herzkammern unumstößlich fest. Diese wird hauptsächlich verursacht durch eine Downregulation von Kaliumkanälen und einen veränderten Kalziumhaushalt in der Zelle.

Bevor weitere Studien zur Mechanotransduktion dargestellt werden, werden in Kapitel 3 zuächst die mechanischen Veränderungen im Herzmuskelgewebe des linken Ventrikels beschrieben, die im AVB-Modell eine Rolle spielen. In seriellen akut-chronischen Experimenten am intakten, kontrahierenden Herzen konnte gezeigt werden, dass end-diastolischer Stress und Strain sowie EjektionsStrain signifikant erhöht sind. Systolischer Stress war nicht erhöht. Diese frühzeitige Zunahme mechanischer Kräfte, vom akuten Moment an bis ungefähr 2 Wochen nach AVB, wurde begleitet von einem Maximum des hypertrophen Wachstums des Herzmuskels. Dies unterstützt die Annahme, dass Stress und Strain eine wichtige Rolle als primäre Reize der Mechanotransduktion spielen. Unser Ansatz, bei dem wir die Echokardiographie und invasive Blutdruckmessungen mit mathematischen Modellen der kardialen mechanischen Kräfte kombinieren, ergibt quantitative Stress- und StrainWerte, die vergleichbar sind mit experimentellen Daten aus isoliertem Herzmuskelgewebe. Somit ist das Extrapolieren von in-vivo Daten zu in-vitro Daten und vice versa möglich, und gehören translationelle Bestimmungen der Mechanotransduktion damit zu den neuen Möglichkeiten. 
In Kapitel $\mathbf{4}$ wird eine neue minimal-invasive transthorakale Methode beschrieben mit der Myokardbiopsien in serieller Weise aus der linksventrikulären Wand entnommen werden können. So kann eine molekulare Analyse der myokardialen Remodellierung in Relation gesetzt werden zu seriellen mechanischen Phänotypisierungen, entsprechend der Beschreibung in Kapitel 3. Diese Methode ermöglicht es, bei denselben Individuen wiederholt Biopsien zu entnehmen, die im Vergleich zu früheren Methoden ein relativ großes Volumen haben. Die Gewebeproben hatten damit eine ausgezeichnete Qualität und waren somit brauchbar für diverse zellbiologische, molekulare und elektrophysiologische Bestimmungen. Im Weiteren wurde deutlich, dass diese Biopsiemethode nach kleinen Anpassungen ausreichend sicher war und außerdem keinerlei Einfluss auf die funktionellen und strukturellen Aspekte des AVB-Herzens hatte. Im Übrigen kann die Methode auch bei anderen Tiermodellen angewendet werden.

In Kapitel $\mathbf{5}$ werden zeitabhängige Aspekte der elektrischen Remodellierung im AVB-Modell beschrieben. Diese Studie wurde durchgeführt, weil allgemein unzureichende Informationen im Hinblick auf zeitabhängige elektro-molekulare Veränderungen in diesem und in anderen arrhythmologischen Modellen vorhanden sind. Es wurde der Verlauf der Downregulation der Untereinheiten KCNO1 und KCNE1 des $\mathrm{I}_{\mathrm{Ks}}$ lonenkanals (slowly-activating delayed rectifier $\mathrm{K}^{+}$current) und dessen $\beta$-adrenerge Sensibilität analysiert. In seriellen Biopsien aus der apiko-lateralen Wand des linken Ventrikels waren innerhalb einiger Tage nach AVB-Induktion mRNA und Eiweiß der lonenkanaluntereinheit KCNO1 signifikant vermindert. Im weiteren Verlauf folgte eine gleichmäßige Abnahme von mRNA und Eiweiß des $\beta$-adrenergen Rezeptors. Im selben Zeitintervall wurden im Blutplasma zeitweise erhöhte Konzentrationen von Noradrenalin festgestellt. In-vivo Studien zeigen eine Repolarisationsinstabilität, die genauer betrachtet den Verlust der Kapazität zur Repolarisationsverkürzung bei $\beta$-adrenerger Stimulation darstellt, wie auch eine Abnahme der Schlag-Schlag Variabilität der Repolarisation. Zum Teil konnte dies durch eine Abnahme der $\beta$-adrenergen Aktivität von $\mathrm{I}_{\mathrm{ks}}$ erklärt werden. Diese Veränderungen fördern das Auftreten ventrikulärer Arrhythmien im Rahmen dieser erworbenen Form der lonenkanalpathologie.

In Kapitel 6 wird näher eingegangen auf die Mechanismen der Mechanotransduktion beim AVBModell durch quantitative Bestimmung der kardialen mechanischen Kräfte (Kapitel 3) und der Expression von myokardialen Schlüsseleiweißen, die eine Rolle bei der Mechanotransduktion spielen. Während der Phase der frühen Zunahme der mechanischen Belastung, die durch eine Zunahme des end-diastolischen Stresses, des Ejektions Strains und der Erhöhung van brain natriuretic peptide (BNP) gekennzeichnet wird, zeigt sich eine zeitweise verminderte Integrität des Zytoskeletts, die zumindest zum Teil ihre Ursache in der Downregulation der Transkription von spezifischen Eiweißen findet. Eine weitere Destruktion des myofibrillären Apparates und des Desmin-Netzwerks trat nicht auf, jedoch wurde eine Reorganisation des Zytoskeletts festgestellt, die von einer Zunahme sogenannter kompensierender Eiweiße, wie Melusin, P-Akt (Protein-Kinase B), P-Glykogen-Synthase Kinase (GSK) und Muskel-LIM-Protein (MLP), begleitet wird. Diese Veränderungen deuten auf eine starke Neigung zu einer kompensierten Form der myokardialen Hypertrophie hin. 
Um diese neuen Erkenntnisse umzusetzen in die klinische Situation, in der Patienten an kardialer Überbelastung leiden, beschreiben wir in Kapitel 7 einen Ansatz, der es uns ermöglicht die mechanischen Kräfte, die für myokardiale Remodellierung relevant sind, direkt am Bett des Patienten zu quantifizieren. Wir berichten über die ersten Erfahrungen mit diesem vollständig nicht-invasiven Modell ventrikulärer Hämodynamik und mechanischer kardialer Kräfte, angewandt bei Patienten mit verschiedenen Formen kardialer Überbelastung. Durch die Verwendung des CircAdapt Computer Modells (entwickelt in der Abteilung für Biophysik des kardiovaskulären Forschungsinstituts CARIM in Maastricht) in Kombination mit transthorakaler Echokardiographie und nicht-invasiver Blutdruckmessung, konnte gezeigt werden, dass die CircAdapt Simulation die Hämodynamik des linken Ventrikels auf eine sehr genaue Art und Weise, vergleichbar mit invasiven Messungen, wiedergibt. Zusammenfassend ist es praktisch möglich, vollständig nicht-invasiv die jeweils patientenspezifische intrakardiale Hämodynamik und ebenfalls die mechanischen Kräfte die auf die Herzmuskelfasern einwirken, modellhaft darzustellen. Dieser Ansatz bietet wichtige klinische Möglichkeiten, um direkt am Bett des Patienten systolische sowie diastolische Parameter, die bei der Überbelastung des Herzens eine Rolle spielen, zu bestimmen. Hierbei geht es vor allem um Druck-Volumen und Stress-Strain Beziehungen, die als wichtige Faktoren der Remodellierung und prognostischen Einschätzung angesehen werden. 


\section{APPENDIX}

\section{Full colour illustration chapter 1}

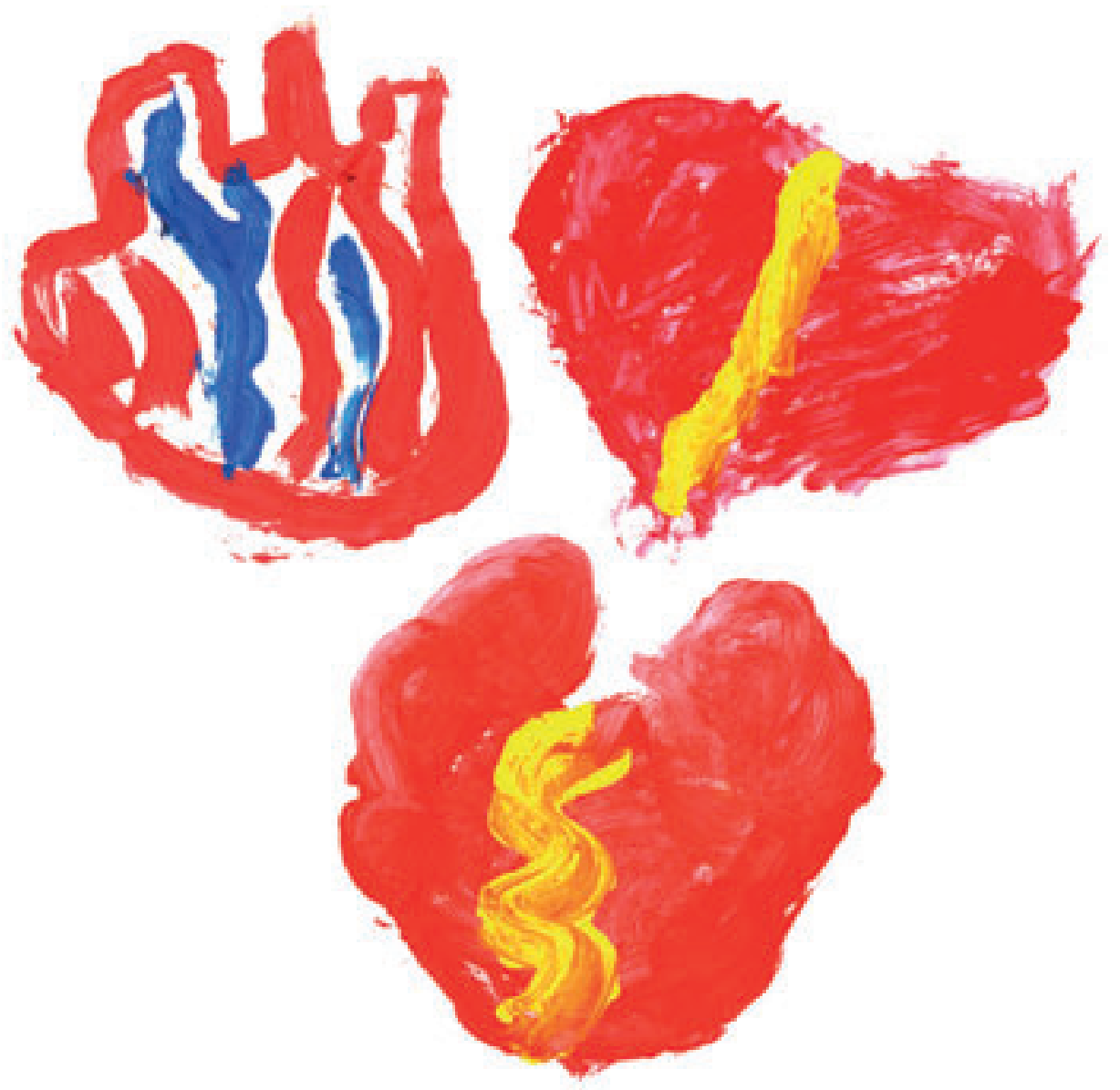

Figure 1 | The heart. Artwork by Aaron (7 years), Eline (5 years) and Marit Donker (3 years). From left to right. 
Full colour illustrations chapter 4

a

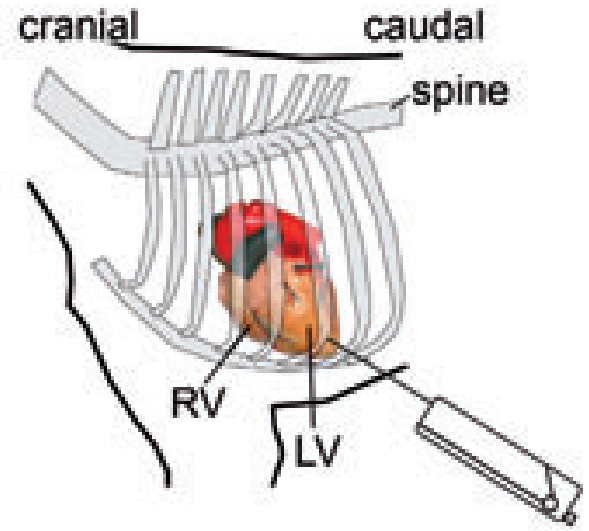

C

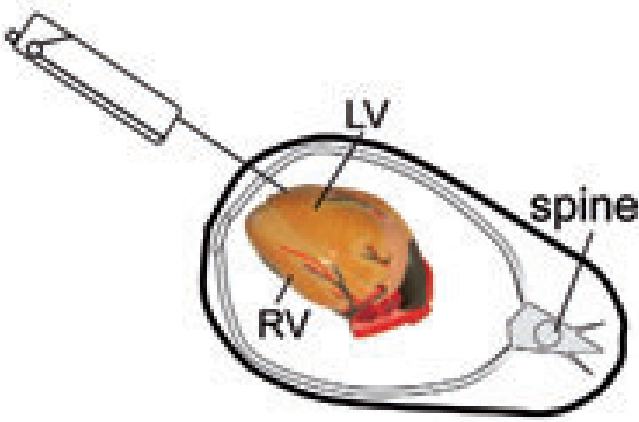

\section{b}

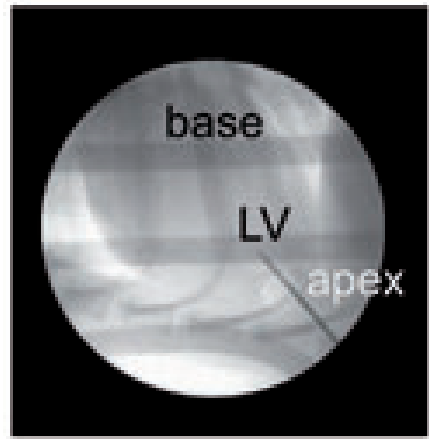

d

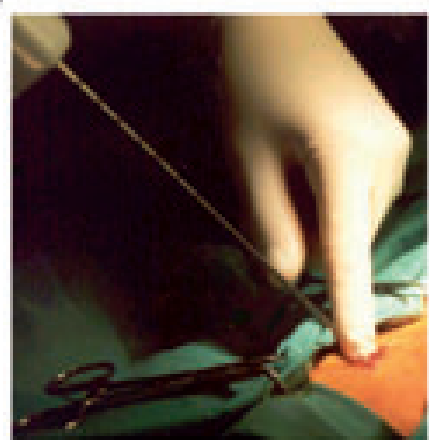

Figure 1 | Serial percutaneous sampling of LV intra-mural biopsies. a Schematic illustration of optimal biopsy needle positioning in the sagittal plane in relation to the intra-thoracic location of the heart in the adult dog. $\mathbf{b}$ Under fluoroscopic guidance, the needle is advanced into the apicolateral LV wall. c Schematic illustration of optimal biopsy needle positioning in the transverse plane (caudal view) in relation to the intra-thoracic location of the heart. $\mathbf{d}$ A small incision facilitates percutaneous introduction of the biopsy needle into the left anterolateral inter-costal space overlying the LV apex. See text for detailed description. 


\section{a}

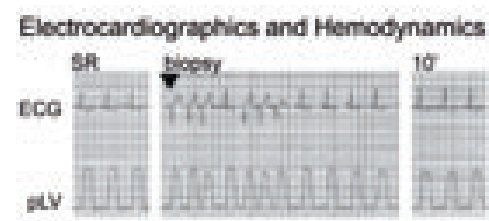

b

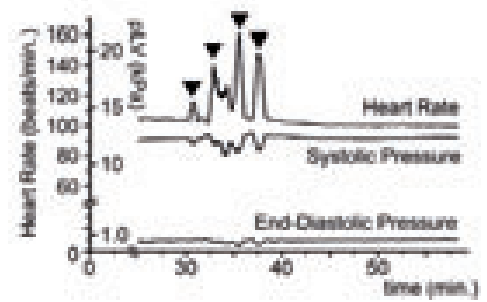

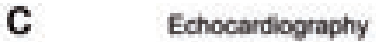
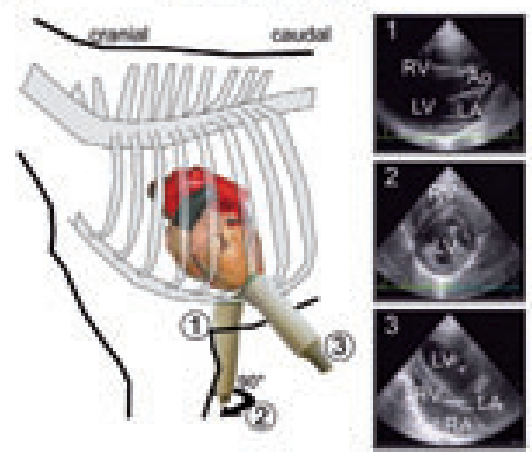

Figure 2 | Procedural monitoring during biopsy sampling. a Electrocardiographic and hemodynamic monitoring shows ventricular extrasystoles (arrowhead) during intra-myocardial insertion of the biopsy needle. SR, sinus rhythm, pLV, intra-cavitary LV pressure. Ten minutes after sampling, the ECG and LV pressure tracings are similar to the baseline (SR). b Monitoring of heart rate and LV systolic and diastolic pressure trends reveals stable tracings after sequential sampling of four biopsies accompanied by multiple ventricular extra-systoles (arrowheads). c Trans-thoracic echocardiographic approach in the adult dog. The right parasternal transducer positions allows long axis (1) and, after rotation, short axis views (2), whereas left parasternal apical scanning provides four-chamber views (3). Note that besides B-mode also M-mode and Doppler-flow measurements (not shown) can be easily performed. Ao, ascending aorta; LA, left atrium; RA, right atrium. 

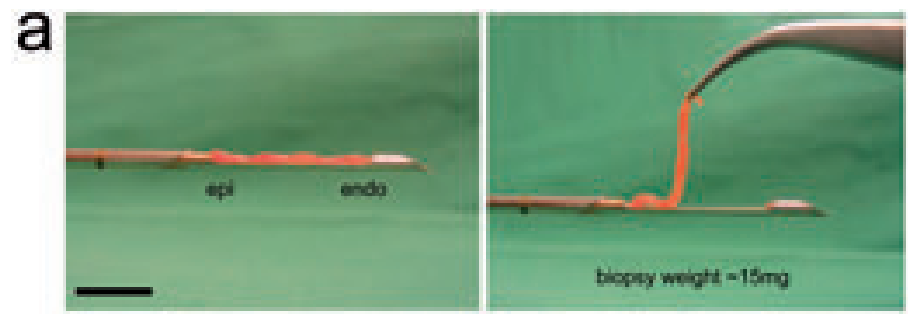

\section{(cryo)sections}

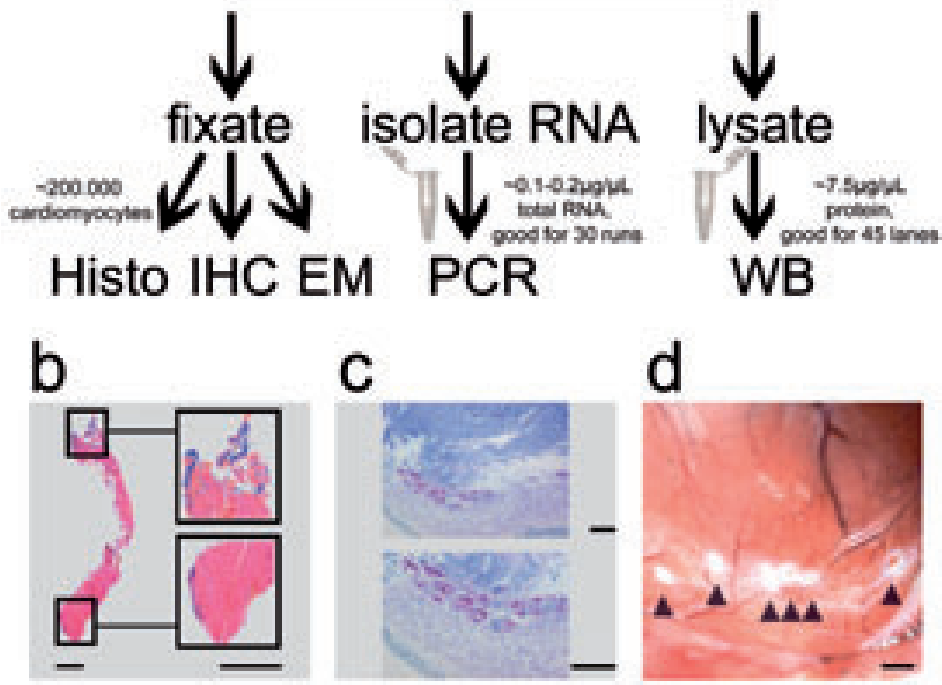

Figure $3 \mid$ Processing of LV myocardial needle biopsies. a Biopsies can be easily lifted from the needle with tweezers. The specimen can be processed as a complete intra-mural cylinder or can be dissected into smaller pieces. After freezing, cryosections can be prepared, allowing simultaneous application of different analytical methods as, e.g., Histo histology, IHC immunohistochemistry, EM electron microscopy, PCR polymerase chain reaction and WB Western blotting. Scale bar indicates $1 \mathrm{~cm}$. b The LM photomicrograph illustrates the good quality of the myocardial samples using Masson's Trichrome staining (cardiomyocytes [red]/fibrous tissue [blue]). This example of an intra-mural biopsy extends from the epicardium (top inset) to the endocardium (bottom inset). Scale bars indicate $1 \mathrm{~mm}$. c Intra-mural extension of the biopsy sample can also be confirmed by visualisation of the (sub)endocardial layers as illustrated in the light-microscopic photograph using toluidine blue counterstained with periodic acid-Schiff; endocardium (pale blue), working myocardium (blue) and glycogen (magenta)containing Purkinje fibres. Scale bars indicate $100 \mu \mathrm{m}$. d At autopsy, only limited scar formation occurred at the sampling sites (arrowheads) after repeated biopsies. Scale bar indicates $2 \mathrm{~mm}$. 
CHAPTER 9 | APPENDIX
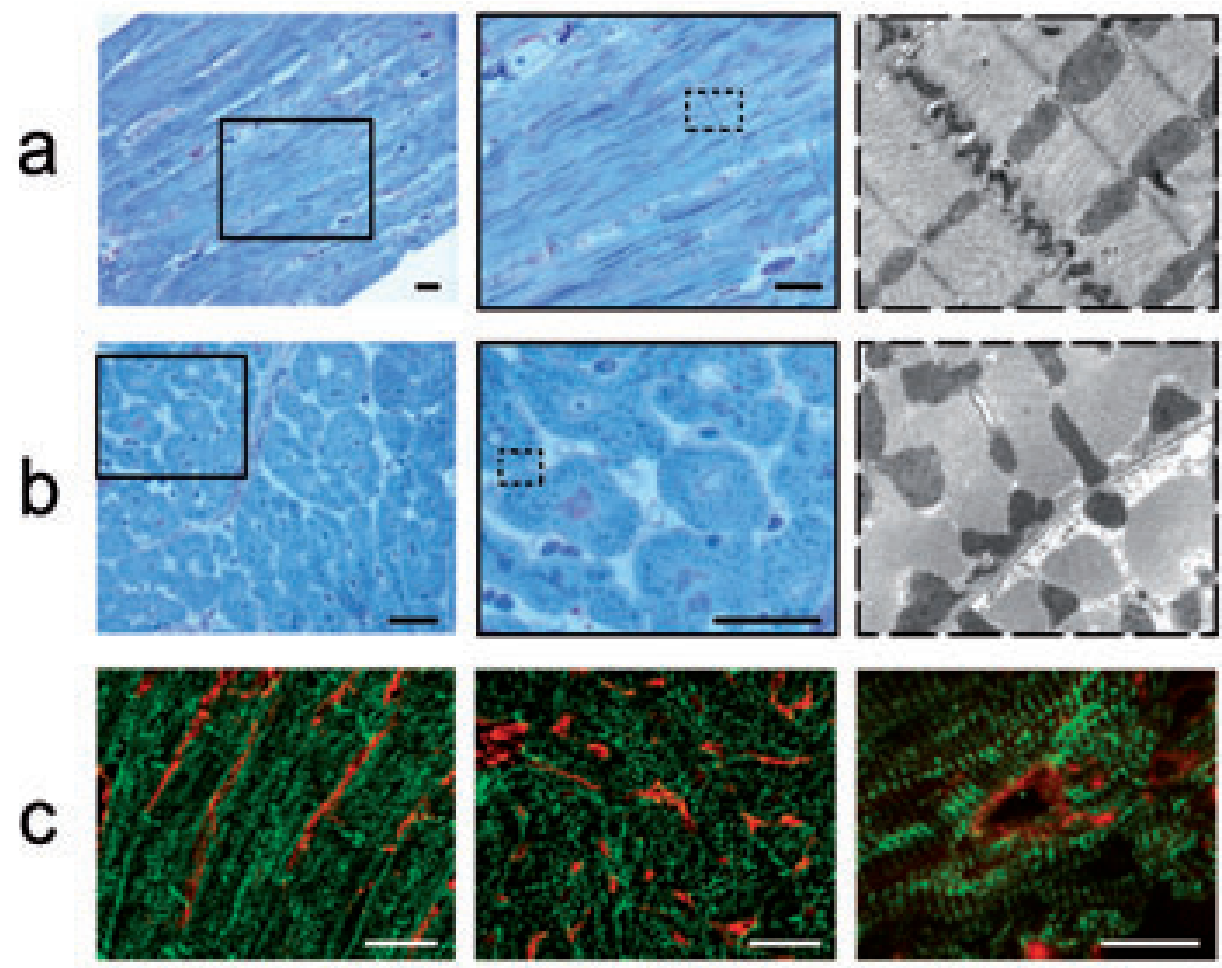

Figure 4 Cell-biological myocardial analysis in LV needle biopsies. a Representative light-microscopic (toluidine blue counterstained with periodic acid Schiff) and electron-microscopic photographs showing longitudinally sectioned cardiomyocytes within a normal myocardial texture from a dog bioptized during SR, demonstrating the excellent tissue quality. Scale bars indicate $20 \mu \mathrm{m}$. b Light-microscopic and electron-microscopic photographs corresponding to a showing cross-sectioned cardiomyocytes. Scale bars indicate $20 \mu \mathrm{m}$. c Immunofluorescent histochemistry in LV myocardium double-labelling the cardiomyocyte intermediate filament desmin (green), the fibroblast intermediate filament vimentin (red; left and middle) and alpha smooth muscle actin present in the vessel wall (red, right), in longitudinally (left, right) and transversally sectioned myocardium (middle). Scale bars indicate $20 \mu \mathrm{m}$. 
Full colour illustrations chapter 6
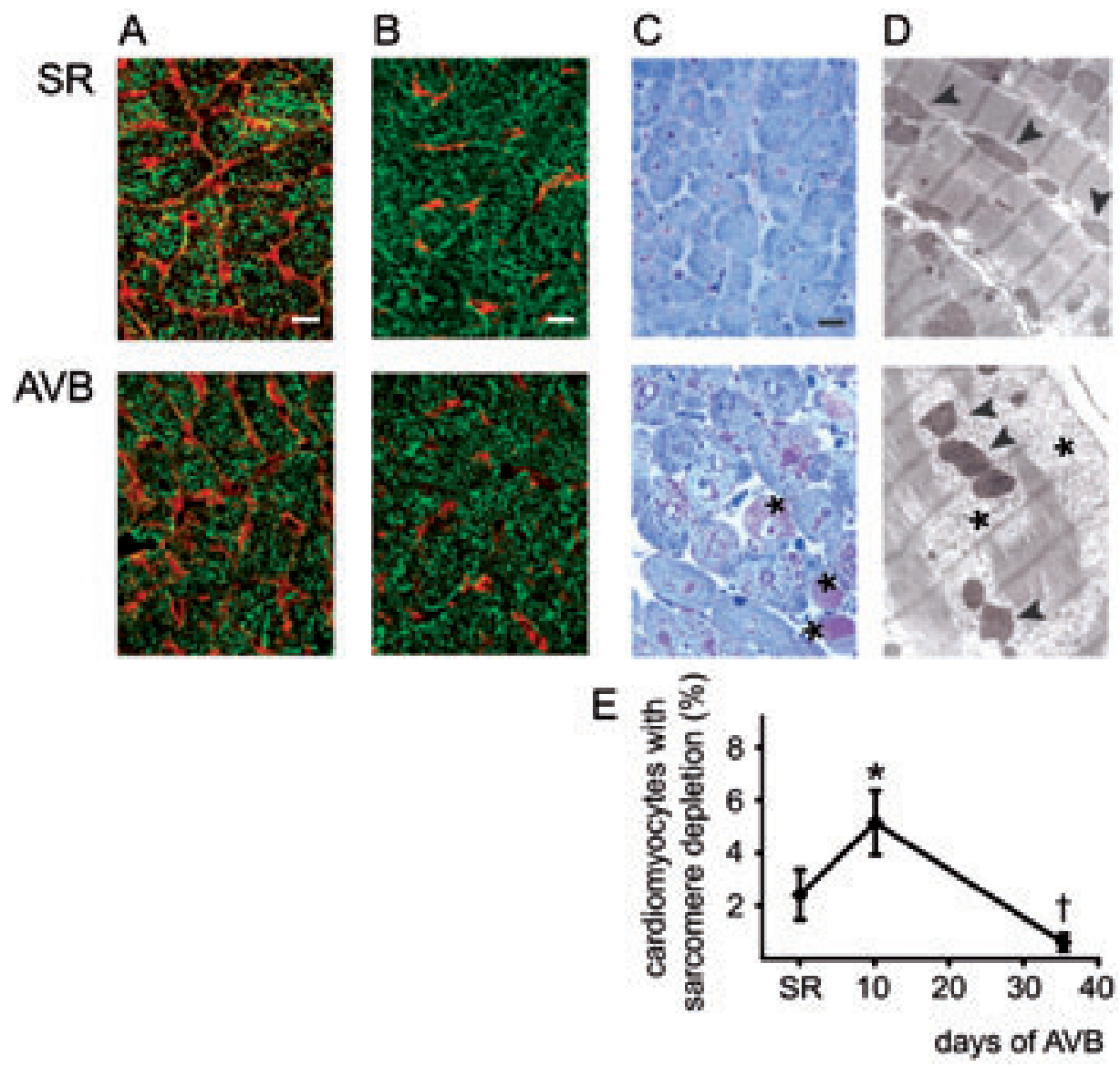

Figure $3 \mid$ Time course of structural remodeling before and after AVB. Immunofluorescent double staining for desmin (green) and laminin (red) A and desmin (green) and vimentin (red) B showing no differences between SR and AVB. C light microscopy (LM) photomicrographs of periodic acid-Schiff-/Toluidine blue-stained left ventricular (LV) myocardium from serial biopsies of one individual dog showing increased cytoplasmic glycogen content during AVB (asterisks), which was absent at SR. D electron microscopy photomicrographs illustrating ultrastructural details, as glycogen accumulation in areas with depletion of sarcomeres and remnants of myofibrils (asterisks) altered mitochondrial shape and size as compared with SR (arrowheads). E relative number of cardiomyocytes showing depletion of sarcomeres. Scale bars in $A-C$ indicate $10 \mu \mathrm{m} .{ }^{*} P<0.05$ vs. $S R ; \uparrow P<0.05$ vs. 10 days AVB. 


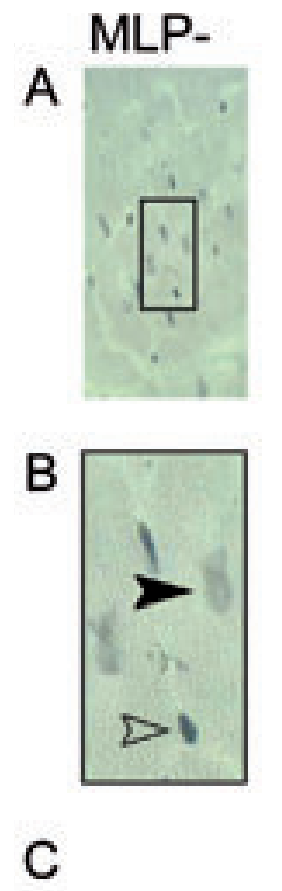

\section{SR}

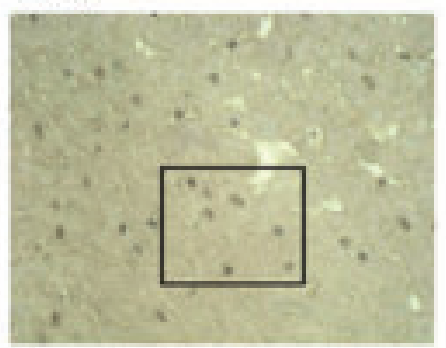

\section{AVB}
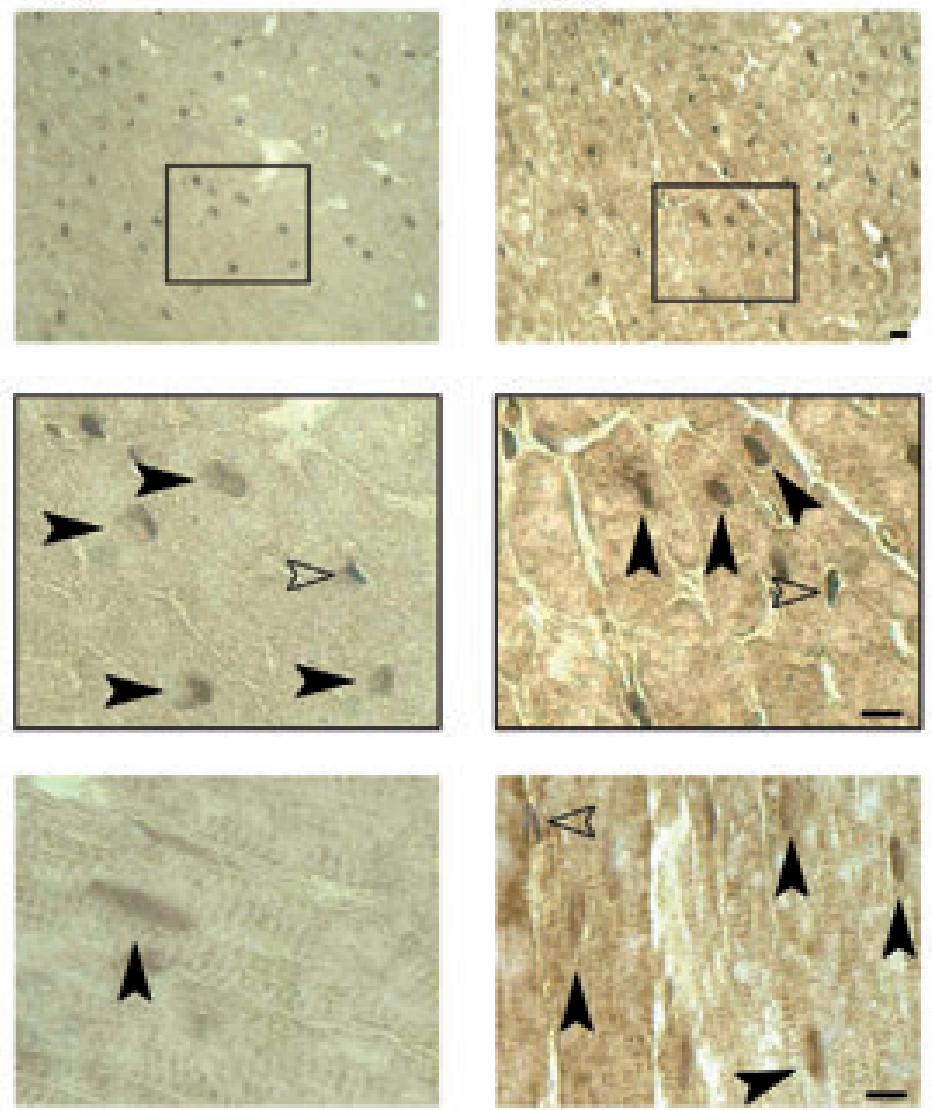

Figure $\mathbf{5} \mid$ Cardiomyocyte expression pattern of MLP before and after AVB. LM photomicrographs show immunohistochemical expression of MLP in LV myocardium at low $\mathbf{A}$ and high $\mathbf{B}$ and $\mathbf{C}$ magnification, in transversely $\mathbf{A}$ and $\mathbf{B}$ and longitudinally $\mathbf{C}$ sectioned cardiomyocytes. At control (SR), a mild MLP expression was observed within the cytoplasm and the nucleus $\mathbf{B}$ and $\mathbf{C}$; solid arrowheads. Note that noncardiomyocyte nuclei $\mathbf{B}$ and $\mathbf{C}$, open arrowhead stain blue, whereas cardiomyocyte nuclei show MLP labeling B and C, solid arrowhead. In negative control (MLP.), both cardiomyocyte nuclei $\mathbf{B}$ and $\mathbf{C}$, solid arrowhead and noncardiomyocyte nuclei $\mathbf{B}$ and $\mathbf{C}$, open arrowheads stain blue, indicating the absence of MLP labeling. During AVB, both cardiomyocyte cytoplasmic and nuclear expression of MLP are increased B and C. Scale bars indicate $10 \mu \mathrm{m}$. 

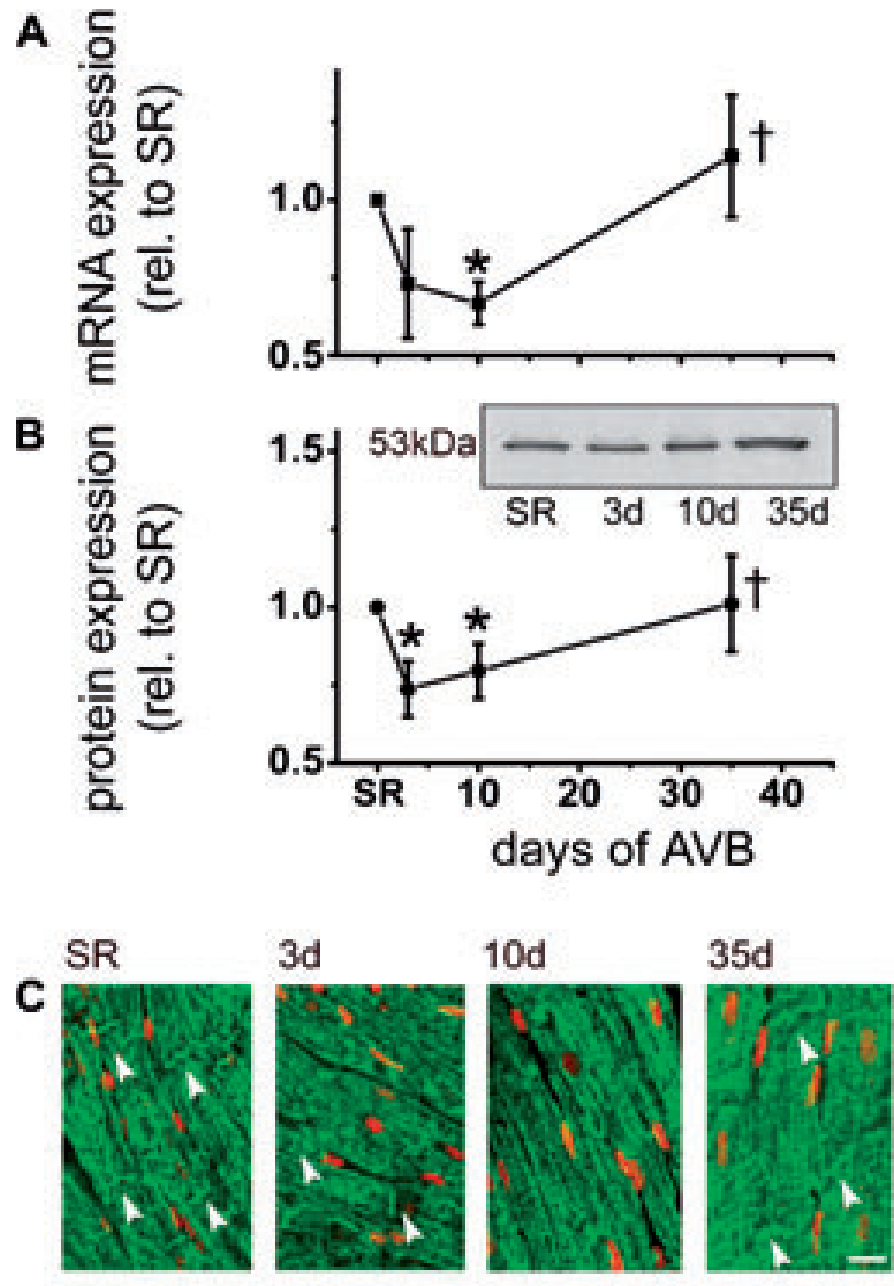

Figure $7 \mid$ Time course of desmin expression before and after AVB. Serial real-time PCR $a$ and Western blot analysis b show average values standardized to $S R$ ( $n=9$

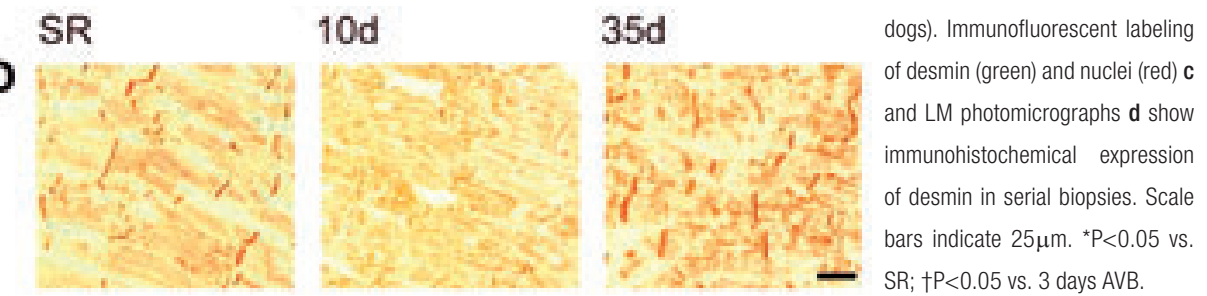


Full colour illustrations chapter 8

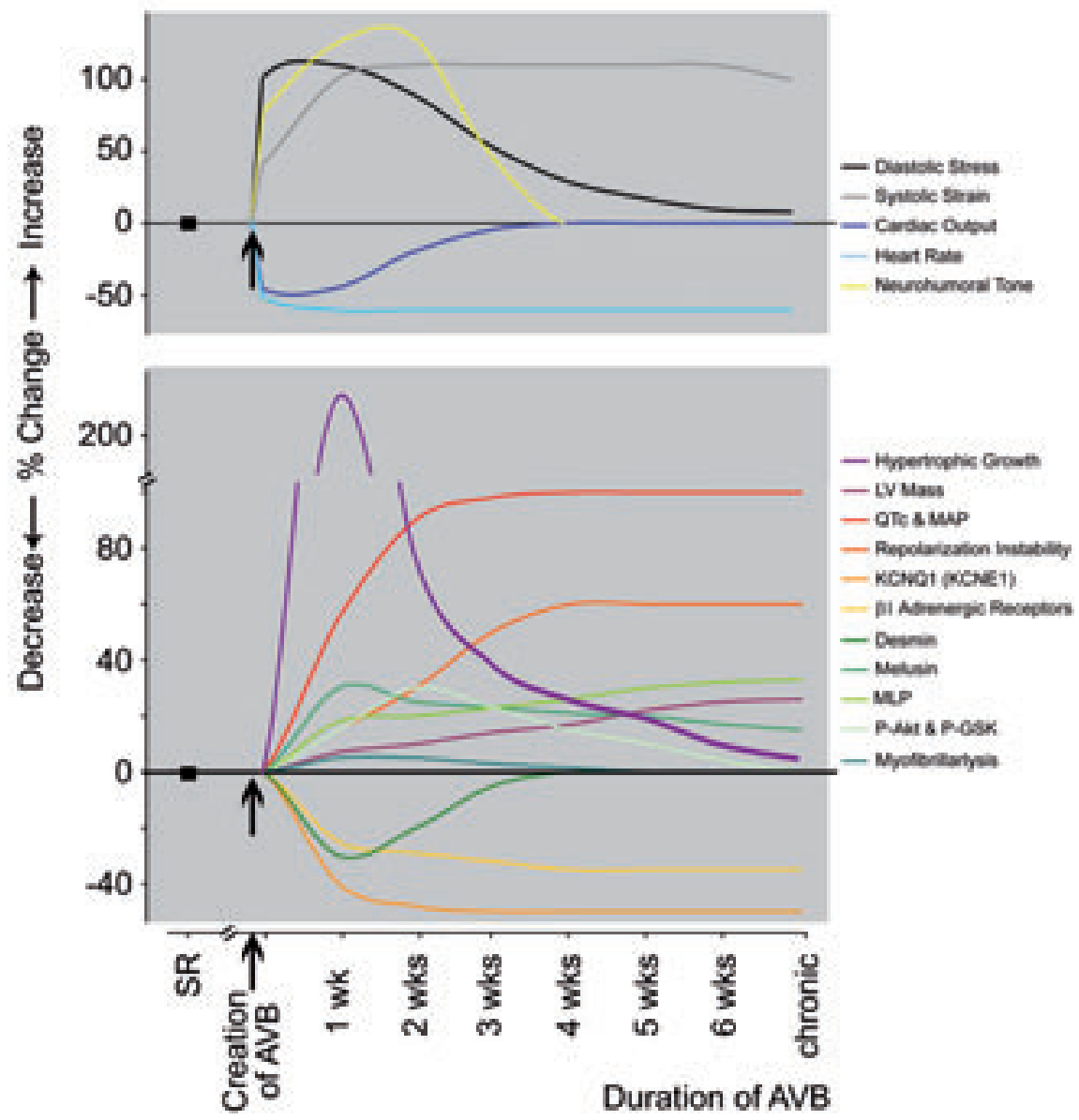

Figure 2 | Time course of cardiac mechanics and related remodeling in the AVB model. Schematic illustration of cardiac mechanics, hemodynamics and neurohumoral tone upon creation of AVB in temporal relation to different functional and structural myocardial remodeling processes. 
Diastole
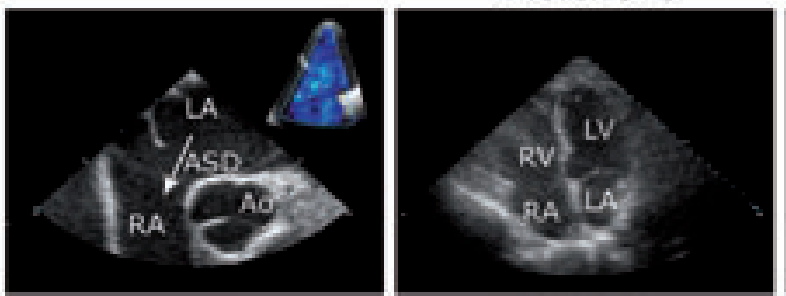

-

Systole

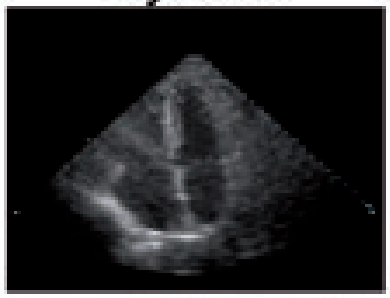

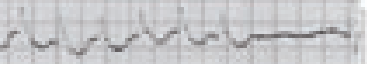

11 in

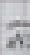

sin-

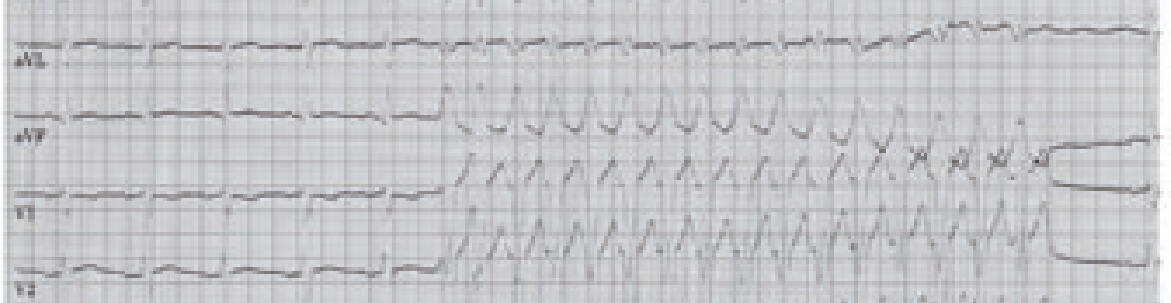

is

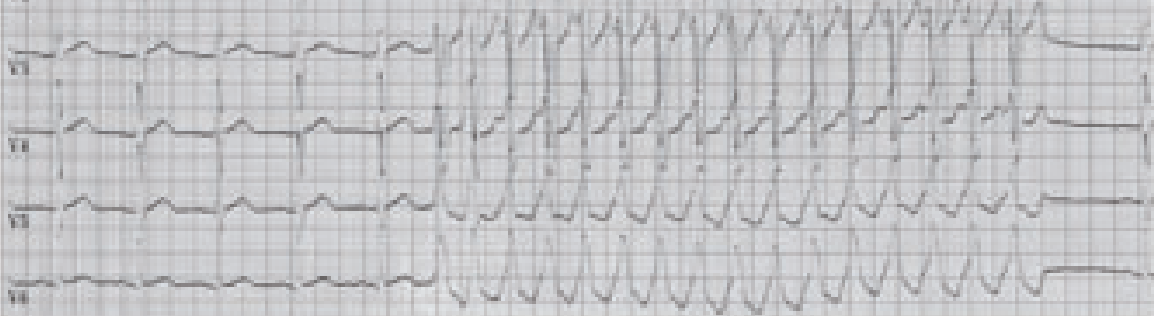

Figure $3 \mid$ Cardiac mechanomyopathy - Case 1. Transesophageal echocardiography (top, left panel) demonstrating ASD type II (arrow) with important left-to-right shunting (insert, color Doppler flow). Ao indicates aortic valve, LA and RA indicate the left and right atrium, respectively. Transthoracic echocardiographic 4-chamber views after surgical correction of the ASD (top, mid and right panel) showing a somewhat dilated RV and a normal LV during diastole (top, mid) and systole (top, right). 12-Leads electrocardiogram (bottom) 10 days after surgical correction of the ASD showing SR (left) and VT (right) originating in the RV outflow tract. See text for details. 


\section{DANKWOORD}

Lieve Birgit, wie kan ik meer danken voor alle steun tijdens dit beproefschrift dan jou?! Jij was in al die jaren mijn beste vriendin. Zonder jou was het onmogelijk geweest om het werk te combineren met een normaal familieleven. Aaron, Eline en Marit, vrolijke noten ... "papa, heb je het boek vandaag echt helemaal klaar gemaakt ?" ... toen papa ook nog erover begon hoe een hart eruit ziet leken jullie er helemaal niets meer van te begrijpen ... maar wat hebben jullie er mooie schilderijen van gemaakt. Overigens, ik maak me geen illusies. Dit hoofdstuk is voor velen het meest populaire stukje proefschrift. Geen probleem, ik ga jullie wel een keer vertellen waar het proefschrift zelf ook nog weer over ging.

Maar nu dan het lang verwachte dankwoord. Als eerste succesvolle survivor van een AGIKO constructie (assistent geneeskundige in opleiding tot klinisch onderzoeker) in de Maastrichtse Cardiologie is het bijna niet mogelijk om iedereen te bedanken die heeft bijgedragen aan al die jaren in kliniek en onderzoeksland. Eén ding is zeker, ik weet wel wie ik niet wil bedanken voor de begeleiding van dit proefschrift en de mogelijkheden voor jonge, enthousiaste artsen om wetenschappelijk onderzoek met klinisch engagement te combineren, namelijk de MSRC (Medisch Specialisten Registratie Commissie). Als je naar Nederland komt en denkt de Duitse bureaucratie te zijn ontvlucht, vind je hem bij de MSRC meer dan driedubbel, en zeker niet gratis, terug. Prof. Hein Wellens, ik wil $u$ dan ook hartelijk bedanken voor de felle, onorthodoxe strijd tegen de instanties (met en zonder toga). Professor Wellens, het succesvolle afronden van dit proefschrift is ook een overwinning voor de translatie beogende opleidingsconstructies. Nogmaals dank voor uw vertrouwen in mijn eerste klinische jaren, de ongelofelijke power in kliniek en wetenschap en dank voor een onvergetelijke en inspirerende historische tijd en vooral ook dank voor het kortste sollicitatiegesprek ooit. Sorry, overigens, nogmaals voor de heerlijke rode wijn op uw mooie witte bankstel.

En dan is er Paul Volders. Paul, jongen, de Y-ruimte is al lang verleden tijd, ik zie je nog zitten aan je oude 486 (66 MHz); amper te geloven maar jij was vele malen sneller dan die processor. Terwijl hij draaide om alles op te slaan had jij al weer een nieuw idee over hoe die ene halve zin nog net iets strakker kon. Daar verdween ook die mooie alinea van zojuist, die mij meer dan een halve middag had gekost, onder die oneerbiedige Delete knop. Nadat ik naar jou was overgelopen, heb je mij meer dan voortreffelijk begeleid in al die jaren. Inhoudelijke steun was vanzelfsprekend en je hebt je uitstekende overzicht over de wetenschappelijke breedte van het vak met mij gedeeld, hetgeen je ook nog eens vertaalt in prachtige visies. Maar, het meest belangrijke is wellicht nog dat de filosofische teneur steeds dieper tot mij doorgedrongen is. Ik kon het nooit geloven, maar een proefschrift schrijven leidt toch echt tot een persoonlijke en professionele vorming. Ik hoop dat we nog veel plezier en succes zullen hebben de komende jaren ....

Prof. Harry Crijns, beste Harry, alhoewel ik nog een van de Altlasten was, heb je mij altijd het gevoel gegeven dat mijn project toch werkelijk iets unieks had. In de drukte van jouw positie heb je veel tijd kunnen maken voor de altijd scherpe en vooral constructieve discussies waarbij jouw uitgesproken enthousiasme prevaleerde. Je hecht veel waarde aan integratie van de wetenschap in 
het echte klinische werk. Als afgestudeerde AGIKO kan ik mij geen betere leermeester wensen. Ik loop nog steeds iedere dag fluitend naar mijn werk (en soms ook zo maar even je kamer op - dank voor de lage drempel) en daaraan heb jij een belangrijke bijdrage. Dank ook voor jouw visie op mijn Intensive Care project.

Prof. Marc Vos, beste Marc, ik heb dan toch gelukkig nog veel van jou geleerd: blijven gaan, nooit omvallen, geen valse bescheidenheid, op het doel af, een commerciële neus, overeind blijven staan in de felste discussies ... ik ken er nog wel een paar ... belangrijke bewapening voor de academische strijd. Marc, ik durf te wedden, jij zou zeker ook een goede bondscoach zijn ... . Ik wil je uitdrukkelijk danken voor je onuitputtelijke enthousiasme en steun in een bewust gekozen rampzalig risicovol project ... volgens mij was jij ook wel eens de kluts kwijt?!

Dr. Emile Cheriex, beste Miel, je blijft toch altijd met een glimlach wat sceptisch kijken als ik over mathematical modeling, myocardial remodeling en dergelijke begin. Gelukkig maar, anders was dit proefschrift er wellicht nooit gekomen. Dank voor je support als klinische opleider in deze altijd soepele AGIKO constructie. Op een gegeven moment wisten we allebei niet meer welk opleidingsschema we nu gingen volgen ... fantastisch toch!? ... dat heeft mij de vrijheid gegeven zo veel te putten uit klinisch en basaal wetenschappelijk werk ... zo is het toch nog een waarlijke AGIKO constructie geworden. De laatste jaren ben ik diep onder de indruk geraakt van jouw succesvolle inzet om de klinische opleiding steeds verder te verbeteren. Ook jouw inspanningen hebben in belangrijke mate bijgedragen aan het fluitend naar het werk gaan.

Prof. Theo Arts, beste Theo, helemaal in het begin heb ik van jou geleerd wat er mis is met wall stress en toen kwam voor mij de openbaring van mathematical modeling. Ik weet nog steeds niet altijd wat er in jouw computer gebeurt, maar wat er uit komt is goud waard voor de clinicus practicus. Dank voor de vele uren op jouw kamer. Jouw jarenlange biofysische inspanningen vormen een belangrijke basis voor ons gezamelijk werk. $\mathrm{Nu}$, op naar de kliniek ... .

Prof. Anton Gorgels, beste Ton, jij bent altijd te enthousiasmeren en nooit te beroerd om even je expertise te delen. Dank voor je support in de begintijd van het project en de introductie in de klinisch-wetenschappelijke wereld.

Dr. Luz-Maria Rodriguez en Dr. Carl Timmermans wil ik danken voor de introductie in de beginselen van de Maastrichtse electrofysiologie en alle support door de jaren heen. Carl, het was een goede tijd in Heugem en Luz-Maria, het ergste van jouw deadlines was, dat ze altijd samen vielen met de Amstel Gold Race.

De leden van de beoordelingscommissie Prof. Maurits Allessie, Prof. Theo Arts, Prof. Jan Glatz en Prof. Walter Paulus wil ik bedanken voor hun bereidheid het manuscript kritisch te beoordelen. A special thanks to Prof. Andrew McCulloch for his willingsness to critically evaluate the manuscript ... and coming all the way over the ocean ... I'm really looking forward to exchange views on integrative systems biology.

Mijn paranymfen, Roel Spätjens en Dr. Khalid Murafi. Roel, het is niet goed met woorden te beschrijven hoe belangrijk jouw bijdrage aan dit proefschrift is ... heb een idee ... schets vaag een figuur of tabel ... kijk over een half uur in je mailbox ... klaar; heb een vraag ... Roel weet de weg 
... met (maar zeker ook zonder) internet ... binnen no time is alles opgelost. Roel, ik kon altijd op jou rekenen ... een rots in de experimentele Cardiologie ... creativiteit, snelheid en perfectionisme ... bovendien nog erg kritisch ... een promovendus kan zich niet beter wensen. Khalid, bester Kalli, ohne Porsche geht's nich' ... ich bin immer mehr überzeugt. Nach TURBOlenten Jahren kommen wir beide langsam wieder zu relativer Ruhe. Dein Impulstrieb und Kreativität kennen keine Grenzen ... guter Freund in allen Lebenslagen und immer weniger Lebensfragen ... ich freue mich auf die kommenden Jahre.

Voor mij was de diversiteit van dit proefschrift één van de mooiste aspecten in al die jaren. Inmiddels is er binnen het gebouw vrijwel geen afdeling meer waarmee ik niet op de één of andere manier heb samengewerkt.

Bovenaan staat de afdeling Celbiologie van en met Prof. Frans Ramaekers. Beste Frans, het cytoskelet breekt menig patiënt op, daar geloven we beiden heilig in ... dus, genoeg te doen de komende jaren ... . Prof. Marcel Borgers, beste Marcel, eigenlijk zou ik nog een rondje moeten draaien, want, hoe zit het nou eigenlijk met de electrofysiologische eigenschappen van de hibernerende cel? Ter geruststelling, ik weet nu wel dat het een lastige vraag is. Fons Verheyen en Marie-Hélène Lenders wil ik danken voor het vele minutieuze celbiologische werk. Sorry, Marie-Hélène, ik dacht echt dat het een speciaal putje was voor oplosmiddelen en ik ken nog wel iemand die dat dacht. En dan er is vooral nog Helma Kuijpers bij de afdeling Celbiologie ... of was het nu Cardiobiologie? Helma, jouw inzet heeft mij, het proefschrift en de hele groep wortels doen slaan in de moleculaire biologie. Western blots for Dummies ... je leert het bij Helma ... het is spannender dan koken met de Chef .... .

De perfecte biotechnische ondersteuning van alle experimenten heb ik te danken aan onze operateurs Monique de Jong, Theo van der Nagel en Jet Beekman. Jet, je wijsvinger staat op het titelblad van the European Journal of Physiology - Pflügers Archiv en ook in hoofdstuk 4, figuur $1 \mathrm{~d}$, dat is een mooie weergave van jouw belangrijke bijdrage aan vele proefschriften binnen de Experimentele Cardiologie, ... altijd een vinger aan de pols. Theo, jij bent operateur, anesthesist, intensivist, technische dienst en hoofd magazijn in één persoon, geweldig ... ik weet dat je geniet van je oude? dag. Monique, dank voor al je inspanningen ... en de eerste biopten ... ik had erg weinig tijd om nog eens rustig binnen te lopen ... jij bent goed op weg om met veel feeling je eigen stempel te drukken op de biotechnische expertise binnen onze afdeling.

Met de oude groep Experimentele Cardiologie/ Elektrofysiologie heb ik geweldige jaren gehad, dank aan Chris, Cora, Jérôme, Jet, Jurren, Marc, Milan, Maaike, Marieke \&t Marieke, Markéta, Morten, Paul, Roel, en Stephan. De nieuwe groep met Annerie, Daniel, Helma, Inge, Jan, Jordi, Matthijs, Monique, Viola, Paul \& Roel is gegroeid tot een afdeling binnen de afdeling. Bij iedere werkbespreking is er wel weer een nieuw gezicht bij ... . Het doet me deugd om te zien hoe het integratieve en translationele pionierswerk z'n vruchten afwerpt.

En nu nog mijn klinische collega 's. Dr. Jurren van Opstal, mijn oudste Maastrichtse vriend. Na onvergetelijke jaren in en buiten de Y-ruimte en op 1068 zou ik graag nog een keer willen terugkomen op het Ardennenoffensief en de Mirabelles. Jurren, je had altijd bemoedigende en lovende woorden 
voor mij. Dat heeft me erg goed gedaan in tijden van veel bloed, zweet en tranen. Bewondering heb ik voor jouw succesvolle klinisch-academische carrière, getrouw aan de echte Jurren-stijl. Dr. Leo HAPPY Hofstra ${ }^{\odot} \ldots$ kun je nu wel remmen ? ... ergens in de Limburgse bossen bij $-20 \%$... eindelijk heb je een fatsoenlijke fiets. Nu ik weer ga trainen heb je de remmen echter niet meer nodig ... niet remmen ... rijden maar ... je weet, er was een tijd dat de Bemelerberg geen naam had ... wacht nog maar even, jongens ... straks heb ik ook een Triple ... en was het maar om jullie je beter te laten voelen ... Bas Bekkers en Bernard Broers dank voor de vele kilometers in en buiten het ziekenhuis, wij moeten weer wat meer naar extramuraal. Bas, dank voor je talrijke initiatieven, de voorbeeldige sollicitatie(-brieven), de privé bedside echolessen en de Intensive Cardiac Care Unit (ICCU). Bernard, dank voor de introductie in de Plastische Chirurg(cardiolog)ie en je (droge) humor ... ik ga wel weer mee fietsen.

Het team van de afdeling Cardio-Thoracale Chirurgie wil ik danken voor de inzet bij alle humane hartbiopten, Prof. Jos Maessen voorop. Jos, je bent altijd te porren voor goede ideeën ... en vooral voor de daadkrachtige omzetting daarvan ... dank voor je wetenschappelijke en chirurgisch-technische expertise.

Alle supervisoren in mijn tijd bij de niet alleen maar beschouwende afdeling Interne Geneeskunde, Dr. Bram Kroon, Roger Rennenberg, Evelien Pijpers, Prof. Peter de Leeuw, Dr. Wubbo Mulder, Dr. Pieter van Paassen en collegae wil ik bijzonder danken voor een uitstekend leerzame en vooral gezellige stage van één ... pardon? twee jaar! Niet te vergeten, Prof. Karl Leunissen mijn opleider Interne Geneeskunde, ... no worries ... de AGIKO constructie is nu echt klaar, dank voor uw inzet en begrip.

Natuurlijk wil ik de verpleging danken op de afdelingen binnen het Hart en Vaat Centrum Maastricht. Dank voor jullie begrip en flexibiliteit op onderzoeksdagen en dank voor de plezierige samenwerking op de klinische dagen. Een speciaal woord van dank gaat uit naar het team van afdeling C3.

Barbara Przybylski en Bianca Bastings, wat is het toch een luxe om goede secretaresses te hebben ... en ook nog altijd goed gemutst.

Für eine prägende Zeit im EFMT (Entwicklungs- und Forschungszentrum für Mikrotherapie) bin ich Dr. Peter van Leeuwen zu viel Dank verpflichtet. P(i)eter, scientist puur sang, ich hab' noch oft an die vielen explorativen biomagnetic field map Stunden zurück gedacht. Die klinische Kardiologie hat mich inspiriert in der Augusta Kranken-Anstalt in Bochum vor allem durch Prof. Birgit Hailer und den Chefarzt der Abteilung, Prof. Michael Wehr. Prof. Birgit Hailer, Allround-Kardiologin der besonderen Art, besten Dank für gute, privilegierte Studentenjahre. Professor Wehr, bin ich froh dass Sie mich in die richtige Richtung weggeschickt haben.

Michael Fiegert, beste Kollegen der Spedition Pieper, in vielen Sommermonaten und Wochenenden war der LKW mein Zuhause. Ein Traum eines jeden Studenten um einen Mercedes Sattelzug über Europas Straßen zu steuern. Nach vielen tausenden Kilometern und x-mal Paris, Marseille, Perpignan, Provence, ... hin und her ... . Danke für's Vertrauen. Es war in vielerlei Hinsicht eine gute Vorbereitung für diese Arbeit ... ich hab' auf ' $m$ LKW in manchen Wochen mehr Lebensweisheit gelernt als in vielen Jahren Studium. 
Marja en Helmut Ritterfeld, Bertine, Peter \& Amélie, Thom, Marx-Jan en Sten. Het is toch maar goed, Helmut, dat in 1972 in München Hennie Kuiper gewonnen heeft, ansonsten wären wir einander nicht über den Weg gelaufen ... danke für all die Fahrten nach Stadtlohn und noch viel mehr. Trainer, wo weht's denn hier ... Kopf runter ... Kette rechts ... tret drauf ... fahr da hin ... bis dir die Kniescheiben wegfliegen ... ich hab' oft die Stoßstange und den linken Fensterholmen vom Passat gesehen und verflucht ... Radrennen ist ... wie das echte Leben. Marja, ik ken geen Nederlander die beter Duits spreekt en schrijft, volgens mij heb je wel een Duits accent ... . Familie Ritterfeld ... what a spirit ... what a family ... .

Mijn schoonouders, Mieneke en Peter Cools-Elie en schoonbroer Luc wil ik bedanken voor de introductie in het gezellige bourgondische Limburgse leven en vooral het avondeten ... ik lust zeker nog wel een goed glaasje rode wijn ... en een kopje koffie, natuurlijk ... .

An dieser Stelle gilt ein besonderer Dank meinen Eltern ... kein Weg zu weit, keine Mühe zu groß; liebe Oma Hildegard und Opa Gerrit ich hab gutes Rüstzeug mitbekommen; euer selbstloser Einsatz in all den Jahren hat mir viel ermöglicht und noch ... en nu möss ock noch moal utschäien ... et is nu wal kloar! 


\title{
PUBLICATIONS
}

\author{
Abstracts \\ Hailer B, van Leeuwen P, Donker DW, Lange S, Rahn N, Wehr M. \\ Hailer B, van Leeuwen P, Donker DW, Rahn N, Lange S, Wehr M. \\ Changes in magnetic field maps at QRS-onset after myocardial infarction. \\ 10th International Conference on Biomagntism, Biomag 96, Santa Fe 1996.
}

Magnetfeldfeldveränderungen zum Beginn des QRS Komplexes bei Patienten nach Myokardinfarkt.

165. Tagung der Rheinisch-Westfälischen Gesellschaft für Innere Medizin, Düsseldorf, Dezember 1995:77.

Van Leeuwen P, Hailer B, Donker DW, Lange S, Wehr M.

Non-invasive diagnosis of coronary artery disease at rest on the basis of multichannel magnetocardiography. PACE. 1998;21(II):908.

Rodriguez LM, Timmermans C, Donker DW, Smeets J, de Groot S, Wellens HJJ.

Evidence of atrial remodeling in patients with chronic atrial fibrillation: Observations following internal cardioversion. J Am Coll Cardiol. 1998;31:333A.

Dispersyn GD, Donker DW, Volders PGA, Ausma J, Vos MA, Borgers M.

Aspects of cardiomyocyte dedifferentiation accompany biventricular hypertrophy in dogs with chronic complete AV block.

Europace. 2000;1:B23.

Donker DW, Maessen JG, Vos MA, Volders PGA, Gorgels AP, Hofstra L, Ausma J, Duimel H, Wellens HJJ, Borgers $\mathrm{M}$.

Does the myocardium in human compensated hypertrophy revert to a dedifferentiated phenotype?

J Am Coll Cardiol. 2001;37: 222A-222A Suppl. A.

Donker DW, Volders PGA, Maessen JG, Hofstra L, Ausma J, Leunissen JDM, Borgers M, Vos MA.

Time frame of myocardial dedifferentiation in canine ventricular hypertrophy.

J Mol Cell Cardiol. 2002;34:A20.

Donker DW, Volders PGA, Borgers M, Vos MA, Arts T.

Increases of systolic fiber strain and end-diastolic fiber stress are the primary local mechanical changes in chronic heart block.

Eur Heart J. 2004;25:555-555 Suppl. S.

Stengl M, Ramakers C, Nabar A, Donker DW, Vos MA, Volders PG.

$\mathrm{I}_{\mathrm{Ks}}$ downregulation in canine ventricular hypertrophy confers the loss of $\beta$-adrenergic-induced shortening of repolarization, favoring proarrhythmia.

Circulation 2004;110:319-320, 1531 Suppl. S.

Donker DW, Spaetjens RL, Ramakers C, Arts T, Ramaekers FC, Borgers M, Vos MA, Crijns HJ, Volders PG. Interacting Z-disk proteins serve as conduit for mechanotransduction in dogs with volume overload.

Circulation 2005;112:U467-U467, 1982 Suppl. S.

Donker DW, Volders PG, Crijns HJ, Arts T.

Non-invasive quantification of complete left-ventricular pressure-volume and stress-strain loops in the human heart - application of the CircAdapt model.

Circulation 2007;116:I|622-II622, 2800 Suppl. S. 


\section{Book Chapters}

Hailer B, Van Leeuwen P, Donker DW, Rahn N, Lange S, Wehr M.

Changes in magnetic field maps at QRS-onset after myocardial infarction.

In: Aine C, Okada YC, Stroink G, Swithenby SJ, Wood CC (Eds.):

Biomag 96. 1999. 467-470. New York: Springer.

\section{Donker DW.}

Quantifizierung kardialer Magnetfeldcharakteristika bei Patienten mit koronarer Herzerkrankung.

Dr. med. Thesis. Essen University, Germany, 2002.

Donker DW, Crijns HJGM, Volders PGA.

Electro-mechanical remodeling in hypertrophy. In: Kohl P, Sachs F, Franz MR (Eds.):

Cardiac Mechano-Electric Feedback and Arrhythmias: From Pipette to Patient. 2005. Saunders Elsevier.

\section{Journal Articles}

Hailer B, van Leeuwen P, Donker DW, Grönemeyer D, Seibel R, Wehr M.

Die Anwendung des Biomagnetismus in der Kardiologie.

Herzschrittmacher. 1995;15,90-103.

Van Leeuwen P, Hailer B, Lange S, Donker DW, Grönemeyer D.

Spatial and temporal changes during QT-Interval in the magnetic field of patients with coronary artery disease. Biomed Tech. 1999;44,139-142.

Donker DW, Volders PGA, Arts T, Bekkers BCAM, Hofstra L, Spatjens RLHMG, Beekman JDM, Borgers M, Crijns HJGM, Vos MA.

End-diastolic myofiber stress and ejection strain increase with ventricular volume overload.

Basic Res Cardiol. 2005;100:372-382.

Stengl M, Ramakers C, Donker DW, Nabar A, Rybin AV, Spätjens RL, Van der Nagel T, Wodzig WK, Sipido KR, Antoons G, Moorman AF, Vos MA, Volders PGA.

Temporal patterns of electrical remodeling in canine ventricular hypertrophy: Focus on $\mathrm{I}_{\mathrm{Ks}}$ downregulation and blunted $\beta$-adrenergic activation.

Cardiovasc Res. 2006;72:90-100.

Donker DW, Maessen JG, Spätjens RL, van der Nagel T, de Jong M, Ramaekers FC, Crijns HJ, Vos MA, Volders PG. Serial left-ventricular biopsy sampling using a minimally invasive trans-thoracic approach in adult dogs. Pflugers Arch. 2007;454:1043-51.

Donker DW, Maessen JG, Verheyen F, Ramaekers FC, Spätjens RL, Kuijpers H, Ramakers C, Schiffers PM, Vos MA, Crijns HJ, Volders PG.

Impact of acute and enduring volume overload on mechanotransduction and cytoskeletal integrity of canine left ventricular myocardium.

Am J Physiol Heart Circ Physiol. 2007;292:H2324-32.

\section{Awards}

Best Oral Presentation Award at the NVVC (Netherlands Society of Cardiology), Ermelo, 2004

Young Scientist Award at the BIOMEDICA congress, Maastricht, 2008 